\title{
A high-resolution growth series of Tyrannosaurus rex obtained from multiple lines of evidence
}

\author{
Thomas D. Carr ${ }^{\text {Corresp. } 1}$ \\ ${ }^{1}$ Department of Biology, Carthage College, Kenosha, Wisconsin, United States \\ Corresponding Author: Thomas D. Carr \\ Email address: tcarr@carthage.edu
}

Background. During the growth of complex multicellular organisms, chronological age, size, and morphology change together in a hierarchical and coordinated pattern. Among extinct species, the growth of Tyrannosaurus rex has received repeated attention through quantitative analyses of relative maturity and chronological age. Its growth series shows an extreme transformation from shallow skulls in juveniles to deep skulls in adults along with a reduction in tooth count, and its growth curve shows that $T$. rex had a high growth rate in contrast to its closest relatives. However, separately, these sets of data provide an incomplete picture of the congruence between age, size, and relative maturity in this exemplar species. The goal of this work is to analyze these data sets together using cladistic analysis to produce a single hypothesis of growth that includes all of the relevant data. Methods. The three axes of growth were analyzed together using cladistic analysis, based on a data set of 1,850 morphological characters and 44 specimens. The analysis was run in TNT v.1.5 under a New Technology search followed by a Traditional search. Correlation tests were run in IBM SPSS Statistics v. 24.0.0.0. Results. An initial analysis that included all of the specimens recovered 50 multiple most parsimonious trees; a series of analyses identified 13 wildcard specimens. An analysis run without the wildcard specimens recovered a single most parsimonious tree (i.e., ontogram) of 3,053 steps. The ontogram is composed of 21 growth stages, and all but the first and third are supported by unambiguously optimized synontomorphies. $T$. rex ontogeny can be divided into five discrete growth categories that are diagnosed by chronological age, morphology, and, in part, size (uninformative among adults). The topology shows that the transition from shallow to deep skull shape occurred between 13 and 15 years of age, and the size of the immediate relatives of $T$. rex was exceeded between its 15th and 18th years. Although size and maturity are congruent among juveniles and subadults, congruence is not seen among adults; for example, one of the least mature adults (RSM 2523.8) is also the largest and most massive example of the species. The extreme number of changes at the transition between juveniles and subadults shows that the ontogeny of $T$. rex exhibits 
secondary metamorphosis, analogous to the abrupt ontogenetic changes that are seen at sexual maturity among teleosts. These results provide a point of comparison for testing the congruence between maturity and chronological age, size, and mass, as well as integrating previous work on functional morphology into a rigorous ontogenetic framework. Comparison of the growth series of $T$. rex with those of outgroup taxa clarifies the ontogenetic trends that were inherited from the common ancestor of Archosauriformes. 
1

2 A high-resolution growth series of Tyrannosaurus rex

3 obtained from multiple lines of evidence

4

5

6 Thomas David Carr

7

8 Department of Biology, Carthage College, Kenosha, Wisconsin, United States of America

9

10 Corresponding Author:

11 Thomas David Carr

122001 Alford Park Drive, Kenosha, WI, 53140, USA

13 Email address: tcarr@carthage.edu 


\section{Abstract}

15 Background. During the growth of complex multicellular organisms, chronological age, size, and morphology change together in a hierarchical and coordinated pattern. Among extinct

17 species, the growth of Tyrannosaurus rex has received repeated attention through quantitative analyses of relative maturity and chronological age. Its growth series shows an extreme transformation from shallow skulls in juveniles to deep skulls in adults along with a reduction in tooth count, and its growth curve shows that $T$. rex had a high growth rate in contrast to its

21 closest relatives. However, separately, these sets of data provide an incomplete picture of the congruence between age, size, and relative maturity in this exemplar species. The goal of this work is to analyze these data sets together using cladistic analysis to produce a single hypothesis of growth that includes all of the relevant data.

Methods. The three axes of growth were analyzed together using cladistic analysis, based on a data set of 1,850 morphological characters and 44 specimens. The analysis was run in TNT v.1.5 under a New Technology search followed by a Traditional search. Correlation tests were run in IBM SPSS Statistics v. 24.0.0.0.

Results. An initial analysis that included all of the specimens recovered 50 multiple most parsimonious trees; a series of analyses identified 13 wildcard specimens. An analysis run without the wildcard specimens recovered a single most parsimonious tree (i.e., ontogram) of 3,053 steps. The ontogram is composed of 21 growth stages, and all but the first and third are supported by unambiguously optimized synontomorphies. T. rex ontogeny can be divided into

34 five discrete growth categories that are diagnosed by chronological age, morphology, and, in 35 part, size (uninformative among adults). The topology shows that the transition from shallow to 36 deep skull shape occurred between 13 and 15 years of age, and the size of the immediate 
37 relatives of T. rex was exceeded between its 15 th and 18th years. Although size and maturity are 38 congruent among juveniles and subadults, congruence is not seen among adults; for example,

39 one of the least mature adults (RSM 2523.8) is also the largest and most massive example of the

40 species. The extreme number of changes at the transition between juveniles and subadults shows

41 that the ontogeny of T. rex exhibits secondary metamorphosis, analogous to the abrupt

42 ontogenetic changes that are seen at sexual maturity among teleosts. These results provide a

43 point of comparison for testing the congruence between maturity and chronological age, size,

44 and mass, as well as integrating previous work on functional morphology into a rigorous

45 ontogenetic framework. Comparison of the growth series of T. rex with those of outgroup taxa

46 clarifies the ontogenetic trends that were inherited from the common ancestor of

47 Archosauriformes.

48 Introduction

Even among large tyrannosaurids, Tyrannosaurus rex is an outlier in terms of its gigantic

50 absolute size (Persons et al., 2019; Snively et al., 2019) and extremely high bite force (Bates and

51 Falkingham, 2012, 2018; Cost et al., 2019; Gignac and Erickson, 2017; Henderson, 2002), which

52 is clear evidence that ontogeny in this species was carried far beyond the limits seen in its

53 immediate relatives (Carr, 1999; Erickson et al., 2004). In recent decades, the details of growth

54 in T. rex have been greatly expanded from studies of morphology (Carr, 1999; Carr and

55 Williamson, 2004; Witmer and Ridgely, 2009, 2010), histology (Erickson et al., 2004; Horner

56 and Padian, 2004; Woodward et al., 2020), functional morphology (Henderson, 2002; Henderson

57 and Snively, 2004; Snively and Russell, 2003; Snively et al., 2006, 2019; Therrien et al., 2005),

58 and mass estimation (Bates et al., 2009; Campione et al, 2014; Hutchinson et al., 2011; Persons

59 et al, 2019). Despite this present heyday of wide-ranging research that has swiftly established $T$. 
60 rex as an exemplar fossil species (Brusatte et al., 2010), a comprehensive growth series that

61 combines size, chronological age, and maturity (i.e., size-independent characters) (Brinkman,

62 1988) is currently lacking as well as criteria from all three axes of growth for defining its

63 primary growth stages (Brochu, 1996; Carr, 1999). At present, the congruence between size, age,

64 and maturity are unknown for T. rex, and, in that context, how the details of its growth differed

65 from that of other tyrannosaurids.

66 The use of quantitative cladistic methodology is the most significant advance in studies

67 of ontogeny in extinct species because it can treat size, age, and maturity as independent

68 variables in a single analysis (Brochu, 1996). This approach has been used to recover growth

69 series in extant (Brochu, 1996; Tumarkin-Deratzian et al., 2006) and extinct (Carr, 2010; Carr

70 and Williamson, 2004; Carr et al., 2017; Ezcurra and Butler, 2015; Frederickson and Tumarkin-

71 Deratzian, 2014; Longrich and Field, 2012) archosaurs, including the tyrannosaurids T. rex (Carr

72 and Williamson, 2004), Albertosaurus sarcophagus (Carr, 2010), and Daspletosaurus horneri

73 (Carr et al., 2017). Although the first cladistic growth series that was published for T. rex was

74 based on a small data set limited to 84 craniodental characters in five specimens, it recovered the

75 ontogenetic progression from a long and low skull to a tall skull that is extensively buttressed to

76 resist the loads from a growth-related increase in bite force (Carr and Williamson, 2004). As

77 used in this introduction, the terms for growth categories of T. rex are based on Carr and

78 Williamson (2004), which recovered the synontomorphies (diagnostic characters) of the juvenile,

79 subadult, and adult growth categories. However, based on the low sample size, this analysis did

80 not (1) capture the nuances of the continuum of growth, (2) include postcranial characters, (3)

81 include size and mass data, and (4) was not calibrated to the chronological age of individual

82 specimens. 
The histology-based study of Erickson et al. (2004) defined five growth categories,

84

namely adolescent, juvenile, sub-adult, young adult, and senescent adult. The definitions of these categories were based on the position of specimens along a logistic growth curve, but they were not defined morphologically aside from the number of Lines of Arrested Growth (LAG) and mass estimates. Therefore, independent criteria of maturity are lacking from this study and so growth categories cannot be diagnosed for specimens that lack a mass estimate and LAG number.

In order to overcome those shortcomings, and to answer the call for nonarbitrary criteria for growth stages in nonavian dinosaurs (Hone et al., 2016), 1,850 craniomandibular and postcranial characters, along with size and age data, were analyzed in 44 specimens that span the ontogenetic spectrum from small juvenile (e.g., LACM 28471) to senescent adult (e.g., FMNH PR2081); eight specimens are histologically aged (Erickson et al., 2004; Horner and Padian, 2004; Woodward et al., 2020). The resulting ontogram was mapped onto the growth curve of $T$. rex that was published by Erickson et al. (2004) to (1) identify the timing of ontogenetic changes, (2) define growth categories based on all of the available evidence; and set previous work into a comparative framework.

Terminology. An ontogram is the ontogenetic equivalent of cladogram, a branching diagram that shows the nested sets of progressively exclusive growth stages, which is analogous to the phylogenetic hierarchy of clades. Individual specimens are positioned at the tips of branches and so are analogues of taxa. A synontomorphy is an optimized character that supports a growth stage (i.e., a node) and is the equivalent of a synapomorphy.

A growth stage is the ontogenetic equivalent of a set that includes a node, its preceding internode, and its corresponding branch; the term refers to the position of nodes along the 
106 ontogram. Growth stages are numbered from the node from which the least mature specimen

107 extends in the ontogram (i.e., closest to the root) to the most mature specimen (i.e., farthest from

108 the root), which is indicated by an arrowhead. In contrast, a growth category corresponds to a

109 group that is made up of several growth stages or just one growth stage. Examples of growth

110 categories used in the results section include small juvenile, large juvenile, subadult, young

111 adult, adult, and senescent adult. These categories, aside from the senescent adult, are analogues

112 of phylogenetic grades.

113 A growth rank is used in the correlation tests where a subset of the total number of

114 growth stages is used; e.g., not all specimens have maxillae so, in a comparison of maxillary

115 tooth counts, only a subset of the total number of growth stages was used. Therefore, growth

116 ranks will be fewer in number than growth stages. For example, if, among 19 growth stages,

117 there are only nine specimens of nine different growth stages with maxillary tooth counts, there

118 are nine relevant growth ranks available for comparison in a correlation test. Maturity in the

119 correlation comparisons refers to either the entire $\mathrm{x}$-axis, where maturity increases away from the

120 origin (from immature to mature), or to the position of a given specimen (immature or mature,

121 relative to the origin and other specimens) among the growth ranks. Maturity also refers to the

122 position of a specimen along the ontogram.

123 A corrected rank (=midrank) was noted on the occasions when data points were tied for

124 the same value (i.e., have equivalent ranks) that were converted into midranks following a

125 conventional and straightforward procedure where all initial scores were ranked and then the

126 mean rank (i.e., midrank) was calculated for each set of tied scores; the midranks replace the

127 initial ranks for use in the correlation tests (Whitlock and Schluter, 2015). Without this

128 procedure, tied ranks will affect the null distribution of a sample, increase the value of 
129 correlation tests, and alter the significance level, and so it is necessary to convert them to

130 midranks (i.e., corrected ranks) to obtain meaningful significance values (Amerise and Tarsitano,

131 2015).

132

133 Goals

134 The specific goals of this study were to: (1) recover the growth series of $T$. rex using

135 cladistic analysis based on an expanded dataset (relative to Carr and Williamson, 2004) that

136 includes chronological age, size, and size-independent cranial and postcranial characters; (2)

137 obtain a synthesis of age, size and maturity data by aligning the cladistic results with a

138 previously published growth curve for T. rex (Erickson et al., 2004); (3) redefine growth stages

139 based on all three axes of growth (i.e., age, size, maturity); (4) evaluate previously published

140 hypotheses of variation and growth changes in T. rex based on the results obtained here; (5) use

141 Spearman rank correlation to quantitatively test hypotheses of congruence between bite force,

142 size, mass, chronological age, tooth count, and abiotic factors with maturity; (6) test the

143 hypothesis that ontogeny recapitulates phylogeny; and (7) test the hypothesis of sexual

144 dimorphism in T. rex (e.g., Larson, 2008).

145 Sequence polymorphism. The presence of sequence polymorphism, or the occurrence of

146 multiple growth patterns in a single taxon, is not explicitly obvious using cladistic ontogeny, the

147 approach used here. The method of Ontogenetic Sequence Analysis (OSA; Colbert and Rowe,

148 2008) is used to identify characters that exhibit sequence polymorphism. The presence of

149 multiple pathways of ontogenetic character change has been reported elsewhere in Amniota,

150 including mammals (e.g., Colbert and Rowe, 2008) and Archosauria (e.g., Griffin and Nesbitt,

151 2016a,b; Griffin, 2018). Ergo, T. rex is almost certainly not an exception to this general pattern. 
152 However, this labor-intensive line of investigation was not pursued here, and is deferred for a

153 later study.

\section{Institutional Abbreviations}

155 AMNH, American Museum of Natural History, New York; BM, British Museum of Natural

156 History, London; BMRP, Burpee Museum of Natural History, Rockford; CM, Carnegie Museum

157 of Natural History, Pittsburgh; CMNH, Cleveland Museum of Natural History, Cleveland;

158 DDM, Dinosaur Discovery Museum, Kenosha; FMNH, Field Museum of Natural History,

159 Chicago; LACM, Los Angeles County Museum of Natural History, Los Angeles; MOR,

160 Museum of the Rockies, Bozeman; NMMNH, New Mexico Museum of Natural History and

161 Science, Albuquerque; OMNH, Sam Noble Oklahoma Museum of Natural History, Norman;

162 RSM, Royal Saskatchewan Museum, Eastend; SDSM, South Dakota School of Mines and

163 Technology, Rapid City; TMM, Texas Memorial Museum, Austin; TMP, Royal Tyrrell Museum

164 of Palaeontology, Drumheller; UCMP, University of California at Berkeley Paleontology

165 Museum, Berkeley; UMNH, Utah Museum of Natural History, Salt Lake City; UWBM, Burke

166 Museum, Seattle; UWGM, Geology Museum, Madison.

\section{Assumptions}

168 For the purposes of this study, it was assumed that the assemblage of $T$. rex, which spans

169 Laramidia for a duration of less than 1.0 million years (Fowler, 2017), was a single

170 nonanagenetic population. The phylogenetic position of $T$. rex shows that it is nested among taxa

171 endemic to Asia (T. bataar, Zhuchengtyrannus magnus) and presumably it dispersed from there

172 to Laramidia no earlier than 67 million years ago (cf. Brusatte and Carr, 2016). Unlike preceding

173 Laramidian tyrannosaurid taxa (Loewen et al., 2013), T. rex was not localized to a single

174 depositional basin during this time of seaway regression and so this widespread taxon is best 
175 regarded as a continuous reproductive network that lacks the conditions (i.e., basins) for

176 directional selection over the last million years of the Mesozoic. To test this assumption, an $a$

177 posteriori comparison between stratigraphic position and relative maturity was made for

178 specimens whose position has been published in the literature.

\section{Materials and Methods}

181 Data acquisition. A character matrix of 1,850 hypothetical ontogenetically variable characters 182 for 44 specimens was compiled in MacClade (Maddison and Maddison, 2005) from first-hand 183 observations of fossils, whereas histological counts of LAGs and some size measurements were 184 obtained from the literature (Erickson et al., 2004; Horner and Padian, 2004). The character list, 185 table of character states for each specimen, and character matrix are in Data S1, Data S2, and 186 Data S3, respectively. All fossils included in this study are accessioned in museum or university 187 collections of the United States that are accredited by the American Alliance of Museums 188 (AAM) or are federal repositories, or both. The non-US institutions - BM, ROM, RSM, RTMP 189 are internationally recognized public trusts. Mass estimates were obtained from several sources

190 (Bates et al., 2009; Hutchinson et al., 2011; Campione et al., 2014; Snively et al., 2019; Persons

191 et al., 2019), but were only used in a posteriori analyses. The characters include binary and

192 multistate transformation series, and the coding approach of Brazeau (2011) was followed for

193 nested but independent characters.

195 Characters. An effort was made to produce an all-inclusive character matrix that drew from as

196 many ontogenetically variable size-independent anatomical domains as possible from the entire 197 skeleton (Brinkman, 1988), including: shape, pneumatization, suture closure and form, muscle 
198 scar relief, limb proportions, subcutaneous surface texture, fenestrae, skull frame, neurovascular

199 foramina and sulci, ornamentation, endocranial space, etc. (Data S1, Data S2). The unusually

200 complete juvenile specimen BMRP 2002.4.1 served as the template for the character matrix, and

201 so the bones missing from it, or characters not drawn from its bones, do not appear in the

202 character matrix, which include the ribs, gastralia, furculum, carpus, manus, and proximal and

203 distal tarsals. Specimens that had multiple states for a given character (e.g., "juvenile" state on

204 the left side, "adult" state on the right) were coded for the mature state.

206 Character polarity. The process of polarizing ontogenetic characters is analogous to that used

207 for phylogenetic characters. Whereas an outgroup taxon is used to establish the plesiomorphic

208 condition in phylogeny, the least mature specimen, or a set of least mature specimens, is used as

209 the point of contrast between the immature state of a character from its mature state (Brochu,

210 1996). The distinction is based primarily on the relative development of features (Brinkman,

211 1988), such as the progression from a bone with a smooth dorsal surface to a bone with a lump

212 on the homologous surface (Brochu, 1996).

213 The juvenile morphotype for tyrannosaurids in general (Carr, 1999) and T. rex in

214 particular (Carr, 1999; Carr and Williamson, 2004) is well established. In those studies, the

215 specimens LACM 28471 and CMNH 7541, both referable to T. rex (Carr and Williamson, 2004),

216 are the most complete juvenile exemplars of the craniomandibular skeleton for comparison with

217 other T. rex specimens. These specimens are small (skull length less than $60 \mathrm{~cm}$ ) and display the

218 nascent state of ontogenetically variable characters (Carr, 1999; Carr and Williamson, 2004). In

219 addition, histological work has shown that LACM 28471 is young - two years old - in terms in

220 chronological age. As an example of the procedure, the two specimens share the possession of 
221 smooth nasal bones in contrast to the coarse condition that is seen in larger, presumably more

222 mature, specimens. Ergo, the smooth condition is coded with a zero, analogous to the

223 plesiomorphic state of a phylogenetic character, whereas the coarse condition is coded with a

224 one, which is analogous to the apomorphic state. Once a character matrix of independent

225 characters is compiled in this way, the presence of an ontogenetic hierarchy is tested by character

226 congruence, just as the hypothesis of a phylogenetic hierarchy is tested in a conventional

227 cladistic analysis of phylogenetic data. A priori it is not known if the sample of specimens, with

228 their different constellations of hypothetical ontogenetic character states, contain a hierarchical

229 ontogenetic signal: if a signal is present in the data, then an ontogram will be recovered; if signal

230 is absent, then a polytomy will result from the proliferation of multiple competing hypotheses.

231 The hypothesis that a single tyrannosaurid taxon, namely T. rex, is present in the Hell

232 Creek Formation and its lateral equivalents is followed here (Carr, 1999; Carr and Williamson,

233 2004; Woodward et al., 2020). As such, several juvenile specimens that are represented by an

234 isolated tooth (e.g., DDM 1863.1) or bone (e.g., RSM 2347.1) were included in the analysis even

235 if $T$. rex autapomorphies were absent from those specimens, but otherwise they compared closely

236 with LACM 28471 and CMNH 7541. Likewise, isolated bones from adults (e.g., CM 9401) that

237 did exhibit T. rex autapomorphies were included in the analysis.

239 Character matrix. An initial character matrix of 1,851 characters and 45 specimens was

240 compiled in MacClade; upon close scrutiny, one transformation series was found to lack

241 hierarchical structure (i.e., all specimens shared the same code) that was discarded, which

242 reduced the number of characters to 1,850. Also, a tibia (MOR 3028) was coded for only one

243 character and so it was excluded from the analysis, reducing the number of specimens to 44. 

were drawn from the phylogenetic literature (Rauhut, 2003; Carrano et al., 2012; Carr et al., 2017; Data S1, Data S2, Data S3), and the rest were based on comparisons made between T. rex specimens, including a subset that was drawn from the ontogenetic literature (Carr, 1999; Carr and Williamson, 2004). Among the phylogenetic characters, juvenile specimens tended to be coded with the plesiomorphic character states, whereas mature specimens were coded with the apomorphic character states. In view of this pattern, the analysis was used to test the hypothesis that ontogeny recapitulates phylogeny. A total of 211 multistate characters were ordered (Data $252 \mathrm{~S} 1)$.

253 Dorsotemporal fossa. The dorsotemporal fossa is an important osteological feature and its 254 anatomical interpretation affects character definition and construction. In a recent publication 255 (Holliday et al., 2019) it was hypothesized that the rostral part of the dorsotemporal fossa that 256 covers the frontal bone in tyrannosaurids was occupied by vascular tissue - not adductor 257 musculature. Five lines of evidence can be brought against this hypothesis. First, the entire surface of the fossa has a coarse, muscle scar-like texture that is continuous between the parietal,

259 frontal, and postorbital; on occasion the rostral edge of the fossa is elaborated into a coarse ridge 260 (e.g., OMNH 10131-1) and the sagittal crest of the frontal is likewise coarse from muscle 261 scarring. As such, the entire surface is an extensive, bowl-like muscle scar that is similar to the 262 large, discrete muscle scars that are seen elsewhere in the skeleton, such as the semicircular scar 263 on the dorsolateral surface of the ischium or the oval-shaped posterior scars of the femur in 264 tyrannosaurids. Therefore, the claim that "the [dorso]temporal fossa does not bear 265 any...osteological feature that clearly identif[ies] it as a muscular attachment" is not true 266 (Holliday et al., 2019: 9). 
Second, the authors point out that the muscles hypothesized to originate from the

268 dorsotemporal fossa (superficial pseudotemporal, external deep mandibular adductor) must wrap

269 rostroventrally around the caudal edge of the laterosphenoid buttress to insert onto the

270 mandibular ramus, forming an acute angle between the origin and the insertion of the muscle

271 (Holliday et al., 2019). The authors claim that this acute angle "would render [the muscles]

272 functionally equivocal if not entirely useless" (Holliday et al., 2019: 10). However, a hairpin turn

273 in a functional cephalic muscle is seen elsewhere in Archosauria, namely the ventral pterygoid

274 muscle that originates from the palate, which in crocodylians "conspicuously wraps around [the

275 dorsal pterygoid muscle] and the retroarticular process [of the mandibular ramus] to attach to the

276 caudolateral surface of the angular" (Holliday and Witmer, 2007: 465; Gignac and Erickson,

277 2017: Fig. 2) and in several clades of living birds it "attaches to the lateral surface of the

278 mandible similar to the condition found in crocodylians" (Holliday and Witmer, 2007: 467).

279 Therefore, there is precedent for an important cephalic muscle that makes a hairpin turn around a 280 bone without the mediating presence of a sesamoid or trochlea (cf. Holliday et al., 2019). In a

281 similar fashion, the superior oblique of the human eye takes an acute turn through a soft tissue

282 trochlea before it inserts onto the eyeball (Agur and Lee, 1991). It is possible that the turn of the

283 adductor musculature in tyrannosaurids is not as extreme as hypothesized: a digital model of $T$.

284 rex adductor muscles that was used to precisely estimate bite forces in the taxon shows neither a

285 sharp nor acute turn from the fossa to the mandibular ramus (Gignac and Erickson, 2017: fig.

286 2B). Even if the digital model is inaccurate, a sharp bend can also be taken by a muscle in the

287 postcranium; for example, in mammals the popliteus originates on the lateral surface of the

288 femur from which it wraps posteromedially onto the posterior surface of the tibia (e.g., Felis

289 domesticus: De Iuliis and Pulerà, 2020; Homo sapiens: Van De Graaff, 1998). 
291 the coronoid process are situated below the caudal region of the subtemporal fenestra of the

292 skull; i.e., below the channel bounded rostrally by the frontal, postorbital, and laterosphenoid,

293 and caudally by the parietal, squamosal, and quadrate. Regardless, the adductor scar in

294 tyrannosaurids extends further rostrally on the dorsolateral surface of the surangular, to a

295 position below the caudal end of the orbital fenestra; the location of the scar shows that it was

296 produced by a muscle or muscles that extended rostroventrally whether they originated from the

297 dorsotemporal fossa or not. Dissections do show that in crocodylians and birds that it is the

298 superficial pseudotemporal and the deep external mandibular adductor muscles (Holliday and

299 Witmer, 2007: fig. 10B, C, D) that are the rostralmost to insert onto the mandible, a condition

300 inherited by tyrannosaurids from their common archosaurian ancestor. Therefore, the

301 identification of the superficial pseudotemporal and deep external mandibular adductor muscles

302 as the rostralmost jaw adductors requires a turn around the shelf formed by the frontal and 303 postorbital.

304 Fourth, the authors observe that "the caudal edge of the frontoparietal fossa (or rostral

305 edge of the dorsotemporal fossa) is angled sharply vertically creating a physical obstacle for a

306 muscle belly to cross, rather than the excavated, concave fossa one would expect to find where a

307 muscle belly was passing" (Holliday et al. 2019: 9). This observation is correct, but it is

308 important to point out that the fossa formed by the frontal and postorbital is bowl-like, bounded

309 medially by the prominent sagittal crest and laterally by a vertical wall formed by the postorbital.

310 Also, in some amniotes, such as mammals, the fossa is convex, so a concave surface is not

311 required to anchor the jaw-closing muscles (e.g., Canis familiaris). The caudal edge of the

312 frontopostorbital shelf (=laterosphenoid buttress) is merely an abrupt plane change that allows 
313 muscles to extend unobstructed ventrally to their insertions. Analogous abrupt edges are seen in

314 the temporal fossae of mammals and turtles above the mandibular fossa. Therefore, an abrupt

315 edge does not constitute an obstacle, rather, it provides a means for a muscle to extend from the

316 origin to reach the insertion.

317 Fifth, the authors point out that the "frontoparietal fossa in some larger

318 tyrannosaurids... are perforated by numerous foramina and erosional pits in the skull roof"

319 (Holliday et al., 2019: 11) as evidence for a vascular structure in the fossa instead of muscle.

320 Neurovascular foramina do penetrate the dorsotemporal fossa of tyrannosaurids, a condition that

321 is seen in other amniotes, such as mammals, where the temporal fossa is unambiguously a large

322 muscle origin. Also, the erosional pits are a part of the overall mottled texture of the

323 dorsotemporal fossa of tyrannosaurids, analogous to the wrinkled surface of the fossa seen in

324 other amniotes; again, mammals provide convenient examples (e.g., C. familiaris). Therefore, for

325 these reasons the vascular tissue hypothesis is rejected here, and the entire fossa on the frontal is

326 instead regarded as the origin for the adductor musculature. This distinction of causal process

327 (i.e., remodeling associated with a vascular structure or a muscle origin) a priori affects how the

328 characters of the dorsotemporal fossa of the frontal are conceptualized or coded and, a posteriori,

329 affects hypotheses when accounting for how the differences seen between different growth

330 stages are integrated with the rest of the adductor surface of origin.

331 Finally, it is worth pointing out that the image showing the adductor muscle insertions

332 onto the medial surface of the postdentary moiety of a specimen of T. rex (MOR 008) in

333 Holliday (2009: fig. 4K) is upside down, which the labels for the insertions do not take into

334 account. This misorientation has resulted in a complete mislabeling of the moiety; also, it far too

335 rostrally positions the insertion of the deep external adductor mandibular muscle, which is 
336 marked by an inflection point at the rostral end of the muscle scar on the dorsolateral surface of

337 the surangular (the ventral edge of the bone in the photograph). The corrected, caudalward

338 position would reduce the angle of the hairpin turn made by the muscle from the cranium to the

339 mandible.

340

341 Size. In order to test the hypothesis that ontogeny is congruent with phylogeny, size characters

342 were divided into discrete states to match their phylogenetic homologues (Brusatte and Carr,

343 2016; Carr et al., 2017). Where possible, skull length (premaxilla to quadrate) was used as the

344 measurement of absolute size; in the absence of complete skulls, the length of the ilium, or,

345 failing that, the femur was used. The length of the ilium and femur have been shown to closely

346 approximate the length of the skull (Currie, 2003), and so they are used here as proxies when a

347 skull length is unavailable.

348 In addition to that, relative size was used only when a specimen is represented by a single

349 bone or a partial skull or skeleton. For example, the isolated lacrimal FMNH PR2411 is smaller

350 than that of CMNH 7541, a juvenile, and so it is reasonable to assume that when complete the

351 skull, of which the single bone was once a part, was smaller than the larger skull. However, an

352 absolute size cannot be given since allometric trends among small juveniles of $T$. rex are

353 currently unknown.

354

355 Analysis. Following Brochu (1996), an artificial embryo was included to optimize the

356 transformation series on the topology; the codes for the artificial embryo were based on the least

357 mature character states seen in juvenile specimens. The character matrix (S3) was analyzed in

358 TnT v. 1.5 (Goloboff et al., 2003; Goloboff and Catalano, 2016) under a driven New Technology 
359 search using the default parameters for ratchet, tree drift, tree fusion, and sectorial search, and 10

360 replicates were run to find a minimum length tree. The trees obtained from these results were

361 then run under a Traditional Search. In the first analysis that included all 44 specimens, 50 trees

362 of 3,099 steps were recovered, and a strict consensus tree recovered only three nodes (Fig. 1A).

363 A systematic approach was taken to identify wildcard specimens by analyzing specimens

364 in order of decreasing completeness (Table 1); i.e., the three most complete specimens (BMRP

365 2002.4.1, FMMH PR2081, MOR 1125) were analyzed together, which recovered a single tree. In

366 the next analysis, the tree buffer was cleared and next least complete specimen (LACM 150167)

367 was added to the analysis and so on. Specimens that resulted in multiple trees (i.e., wildcards)

368 were identified and excluded from subsequent analyses (Table 1). Several specimens initially

369 resulted in multiple equally parsimonious trees, but later resulted in a single tree after additional

370 specimens were included. Two rounds of this process identified 13 wildcard specimens (Table 1)

371 that ranged in completeness from $6.1 \%$ to $0.2 \%$ of characters coded (i.e., over $90 \%$ missing

372 information). Specimens that were coded for less than $1.5 \%$ of the characters resulted in multiple

373 topologies, indicating the lower limit of information content on topological resolution in this data

374 set (Table 1).

375 In the end, the remaining 31 specimens resulted in a single most parsimonious tree of

3763,053 steps, which was then tested under a Traditional search using TBR branch swapping in

377 case a more parsimonious tree island was missed by the New Technology analysis (Fig. 1B).

378 This analysis returned a single most parsimonious tree of the same topology. Descriptive tree

379 statistics and the apomorphy list were obtained by running the analysis under a heuristic search

380 in PAUP (Swofford, 2002) under ACCTRAN optimization with the tree length and tree topology

381 (assembled in MacClade; Maddison and Maddison, 2005) constrained. 
383 Artificial adult. An attempt to identify the most mature specimen of the sample was made by

384 adding an artificial adult to the character matrix using the following procedure: the ontogram

385 obtained from the TnT analysis was reconstructed in MacClade (Maddison and Maddison, 2005)

386 and the character trace tool was used to display the optimization of each transformation series on

387 the topology. Since the ontogram follows a gradient from least mature (toward the root) to most

388 mature (away from the root), the node furthest from the root served as a point of reference. The

389 furthest node splits into two subgroups (UMNH $11000+$ UCMP $118472+$ MOR 008 on the one

390 hand, and FMNH PR2081 + MOR 2822 + UWGM 181 on the other; Fig. 1B), and, a priori, it

391 could not be determined which group is more mature than the other. Therefore, the artificial

392 adult was coded based on the character state(s) optimized at the internode supporting that group.

393 For example, if the internode was optimized as " 1 ", then the artificial adult was coded

394 accordingly; if it was optimized ambiguously as " 0 " and " 1 ", the artificial adult was coded with

395 both states.

396 Once the codings for the artificial adult were completed, the analysis was again run and

397 the resulting sister specimen of the artificial adult was identified as the most mature specimen in

398 the sample. That specimen would then function as the terminal exemplar of the growth series and

399 its autontomorphies (i.e., individual variation) would be considered to represent the last changes 400 in growth.

401

402 Statistical tests. Statistical tests were completed by using the licensed software package IBM

403 SPSS Statistics version 24.0.0.0. (IBM, 2016). For each Spearman rank correlation comparison,

404 the growth series ranks were compared with ranks converted from each data set; the variables 
405 were treated as ordinal, and a two-way Spearman test was run. For data sets where congruence

406 was seen among juveniles and subadults, but not among adults, a separate test was run for the

407 mature specimens that excluded the immature specimens.

408

409 Chronological age data. Four sources from the literature were used to obtain chronological age

410 for eight specimens (Erickson et al., 2004; Erickson, 2005; Horner and Padian, 2004; Woodward

411 et al., 2020). The overall age estimates of Horner and Padian (2004) were used. The

412 chronological age of BMRP 2002.4.1 is based on Woodward et al. (2020), which reported a

413 higher estimated age of 13 years than the earlier published estimate of 11 years (Erickson, 2005);

414 the Woodward et al. (2020) estimate was based on a wider sampling of the skeleton (e.g., femur

415 and tibia) than that of Erickson (2005), which sampled the fibula. The chronological age of 14

416 years for MOR 555 (Horner and Padian, 2004), an unambiguous adult, was excluded from the

417 comparisons made here because this underestimate was based on a damaged bone (J Horner, 418 2010, pers. comm.).

419

420

\section{Results}

421 Cladistic analyses. The reduced cladistic analysis of 31 specimens, following the New

422 Technology and Traditional searches, recovered one most parsimonious tree (i.e., ontogram) of

4233,053 steps, with a Consistency Index (CI) excluding uninformative characters of 0.65, a

424 Homoplasy Index (HI) of 0.35, a Retention Index (RI) of 0.72, and a Rescaled Consistency Index

425 (RCI) of 0.50 . The ontogram recovered 21 growth stages; in the adult region of the tree, several

426 branches contain multiple specimens; these are most simply interpreted as specimens of the same

427 maturity (Fig. 2). Bremer, jackknife, and bootstrap values are shown in Fig. 1B. 
The ontogram is composed of 21 growth stages, including the group of most mature

specimens, and all but the first and third are supported by unambiguously optimized synontomorphies (Data S4). Despite the addition of 26 specimens and 1,766 characters, these results are congruent with those obtained by Carr and Williamson (2004), where LACM 28471, CMNH 7541, LACM 23845, AMNH FARB 5027, and LACM 23844 were again recovered at progressively mature growth stages (Figs. 1B, 2). MPTs that, in a strict consensus tree, did not group with a single, presumably most mature, specimen. Therefore, a different approach was taken, specifically the greatest distance from the root. Of that group, FMNH PR2081 possessed the greatest number of autontomorphies, 19 character changes, in contrast to the five others; ergo, that specimen was regarded as the most mature of the group, having undergone the greatest amount of change, and so represents the twenty-first growth stage (i.e., the terminus of the growth series). In contrast, the massive specimen RSM 2523.8, previously regarded as the most mature individual T. rex (Persons et al., 2019), was recovered as one of the least mature adults.

The two purported female specimens (BMRP 2006.6.4, MOR 1125), a sex identification based on the presence of femoral medullary bone (Schweitzer et al., 2005; Woodward et al., 2020; this assessment has been challenged by O’Connor et al., 2018), were recovered as a subadult and a young adult, respectively (Fig. 2). Sexual dimorphism was presumed here to be

447 expressed in one of two ways: either the ontogram would split into separate male and female 448 branches, or sexually diagnostic characters might be optimized as individual variation. In the first instance, a split of the ontogram into separate branches is not seen. In the second, the individual variation of MOR 1125 was compared with that of other specimens. 
451 Sexually informative variation, in this case female variation, was assumed to be expressed as

452 multiple homologous character changes seen in MOR 1125 and a repeated set of specimens (i.e.,

453 the identical set of specimens for each shared homologous character change). The comparison

454 found eight homologous character changes shared between MOR 1125 and five other specimens;

455 however, the character changes are not shared with a uniform set of specimens (Table 2).

456 Therefore, there is no skeletodental evidence for sexual dimorphism in the data set (i.e., males

457 and females are skeletally identical aside from the presence of medullary bone); otherwise, the

458 ontogram would have divided into separate male and female branches or homologous character

459 changes would have singled out MOR 1125 and an associated set of specimens. This test is not

460 dependent upon MOR 1125 being a female (O'Connor et al., 2018); if in actuality it is a male,

461 then the set of shared characters would be evidence of that sex.

462

463 Wildcard specimens. Thirteen wildcard specimens were identified; in each case, the analyses

464 that included them resulted in multiple trees that collapsed into one or several polytomies; the

465 regions of collapse are enclosed by ellipses in Figure 1B. Upon comparison of the polytomies,

466 the wildcard specimens could be divided into two groups, where resolution was lost either

467 rootward or distalward relative to BMRP 2002.4.1, the most complete specimen in the dataset;

468 i.e., that specimen was not part of either polytomy. The partial adult specimen, CM 1400, is

469 shown in a polytomy with two other specimens at the end of a branch (Fig. 1B); however,

470 structure was lost elsewhere in the ontogram when this specimens was included in the analysis,

471 and so it was excluded from the backbone topology (Figs. 1B, 2).

472 Despite the fact that the wildcards are coded for less than $7 \%$ of the characters, some

473 occupy a relatively precise location of the ontogram (e.g., CM 1400, CM 9401, DDM 35.1, 
474 MOR 3044, MOR 2925, RSM 2347.1), whereas others collapse entire regions (e.g., BMRP 475 2001.4.70, DDM 1536.1, DDM 1562.14, DDM 1863.1, FMNH PR2411, MOR 002, TMM 476 41436-1). Of these specimens, many are single bones (BMRP 2001.4.70, DDM 35.1, DDM 477 1536.8, FMNH PR2411, MOR 2925, MOR 3044, RSM 2347.1, TMM 41436-1) or teeth (DDM 478 1562.14, DDM 1863.1), whereas only two are a partial skull (CM 1400) or skull and skeleton 479 (MOR 002).

481 Overview of Frequencies of Growth Changes

482 Influence of specimen completeness. The effect of specimen completeness upon the number of 483 synontomorphies at each node was quantified through a Spearman rank correlation test.

484 Examination of the raw data as histograms with a fitted normal curve showed they are positively 485 skewed, and a Shapiro-Wilk test of normality found that they were not normally distributed 486 (completeness, $p=0.001$; synontomorphies, $p=0.000$ ) and so the data were normalized by 487 converting them to ranks (Table 3, Fig. 3). A Shapiro-Wilk test of normality found that the 488 ranked completeness and ranked number of synontomorphies were normally distributed $(p=$ $4890.217,0.134$, respectively). A Spearman rank correlation test resulted in a nonsignificant $(p=$ $4900.423)$ correlation coefficient $\left(r_{s}=0.149\right)$, indicating that the peaks of synontomorphies at each 491 node are not dependent upon the completeness of the specimens that extend from each branch. 492

493 Synontomorphy trends. The overall distribution of growth changes is unimodal (Fig. 4): the 494 highest number occurs early, peaking at the subadult growth category, which then precipitously 495 drops, aside from several low peaks in the adult growth stages. In total, there are five peaks, and 496 four of them mark the onset of growth categories. The peak at growth stage 6 marks the 
497 beginning of the subadult growth category, where an increase in the height of the skull frame and

498 inflation of the bones that enclose the antorbital sinus are seen, as well as changes to the pectoral

499 girdle and limb, and pes. The peak at growth stage 8 marks the onset of the young adult growth

500 category. The peak at growth stage 13 marks the onset of adulthood, where the External

501 Fundamental System (EFS; a narrow band of lines of arrested growth that indicate near cessation

502 of appositional growth) is first seen as well as changes to the antorbital sinus system and the

503 origin of the adductor musculature. The peak at growth stage 19 marks extensive changes to the

504 skull and postcranial skeleton, but by itself does not define a new growth category. Finally, the

505 peak of changes at growth stage 21 correspond to the extensive changes in the skeleton of

506 FMNH PR2081, the most mature and second-best sampled specimen in the data set.

507 Given their abundance in the character matrix, nonphylogenetic synontomorphies are

508 more numerous at each node than phylogenetic synontomorphies, but they broadly follow the

509 same pattern of peaks and valleys (Fig. 5), aside from growth stages 6, 8, 10, and 21, where

510 phylogenetic changes tend to decrease in frequency whereas nonphylogenetic changes increase.

511 This difference in frequency distribution shows that the number of phylogenetic changes is not

512 controlled by the number of characters scored. Phylogenetic changes are frequent (i.e., more than

513 five) early in growth (stages 2 to 9), whereas they are less common among adults (stages 10, 12,

51413,18 to 21 ); if ontogeny is congruent with phylogeny then this gross pattern should be expected

515 since fewer phylogenetic synontomorphies should occur among adults, which presumably would

516 only express characters at the level of species.

517 A comparison of the frequency of cranial and postcranial changes shows that cranial

518 synontomorphies are the most frequent (Fig. 6). Both sets of changes follow the same general

519 pattern; cranial changes are dominant in terms of number and the pattern of the frequency 
520 distribution, whereas postcranial changes do not always occur. Cranial and postcranial changes

521 are most frequent at growth stage 6 , and thereafter they show less than 22 changes per growth

522 stage. This indicates that once the adult morphotype (i.e., tall skull, inflated antorbital sinuses) is

523 achieved, the rate of change, as shown by the number of changes per growth stage, greatly

524 decreases. During adulthood, cranial changes generally outnumber postcranial changes, which

525 tend to cease altogether. Cranial changes are nearly continuous and have five peaks, whereas

526 only three are seen postcranially that are often preceded and followed by one or more growth

527 stages of no change. The absence of postcranial changes in growth stage 5 is an artifact of the

528 least mature specimens lacking postcranial material. The abundance of postcranial changes at

529 growth stage 7 is an artifact of the exemplar specimen having an incomplete skull and a

530 relatively complete hindlimb. In contrast, the absence of postcranial change seen from growth

531 stage 9 to 12 reflects a signal of quiescence given that several specimens in that interval do

532 include postcranial bones.

533 When the frequencies of changes to the cranium and mandible are compared, both follow

534 the same overall pattern (Fig. 7), but the cranial changes generally outnumber those of the

535 mandible. Mandibular changes occur in a series of three low peaks (i.e., less than 8

536 synontomorphies per growth stage) throughout ontogeny; the highest number of changes (7)

537 occurs early in the $5^{\text {th }}$ growth stage, but this trend does not continue into the sustained and

538 extremely high number of changes seen in the cranium at the $6^{\text {th }}$ stage. This difference results

539 from the absence of mandibular bones in the sample of subadults at the fifth stage. In contrast,

540 the exemplar of the seventh growth stage, LACM 23845, is represented by an incomplete

541 mandibular ramus, but, notably, it does not result in mandibular synontomorphies at that growth

542 stage; presumably those changes occurred earlier, at growth stage 5. The absence of changes at 
543 the $7^{\text {th }}$ growth stage indicates that the completion of the progression of changes in the mandibular

544 ramus, which produces the dorsoventrally deep skull frame, precedes that of the cranium. In

545 adulthood, the number of changes in the cranium exceeds that of the mandible or, on occasion,

546 mandibular changes exceed cranial changes at growth stages where the cephalic skeleton is

547 represented solely by mandibular bones (BM R7994) or very few cranial bones (NMMNH P548 3698).

549 Changes to the skull and jaws were examined by anatomical domain, which includes

550 discrete regions of the skull such as bony structures associated with the antorbital air sac system

551 or aggregates of functional structures, such as joint surfaces. For ease of comparison, the

552 domains were separated into two groups: pneumatic systems on the one hand, and apneumatic

553 features on the other. The pneumatic systems include the antorbital sinus system (invades the

554 snout), tympanic sinus system (invades the lateral surface of the braincase), median pharyngeal

555 sinus system (invades the sphenoid rostrum and basicranium), and the cervical air sac system

556 (invades the occiput). In descending order, the most frequent pneumatic changes are associated

557 with the antorbital, cervical (=subcondylar) and tympanic sinus systems, and the median

558 pharyngeal sinus system (Fig. 8). Changes associated with the antorbital sinus occur throughout

559 ontogeny, whereas cervical changes begin in adulthood and continue almost to senescence; in

560 contrast, median pharyngeal changes are limited to growth stage 12 , at the end of the young adult

561 growth category. Finally, tympanic changes are limited to growth stages 9 to 12, during young

562 adulthood.

563 Apneumatic domains include joint surfaces, muscle scars, the subcutaneous surface,

564 neurovasculature, the occiput, dentition, and the skull frame. The skull frame sees the most

565 changes throughout the growth series (Fig. 9). Change to all other domains are low in frequency 
566 (i.e., fewer than 10 changes per growth stage), but most of them also change throughout growth,

567 including joint surfaces (growth stage 4 to 21), the dorsotemporal fossa (growth stage 4 to 21),

568 neurovasculature (growth stage 2 to 21), muscle scars (growth stage 5 to 21), the subcutaneous

569 surface (growth stage 5 to 21). A limited pattern is seen in the dentition where changes occur

570 from the $5^{\text {th }}$ to $17^{\text {th }}$ growth stages. In contrast, nonmuscular and apneumatic changes to the

571 occiput are limited to the young adult growth category (growth stage 9).

572 In the postcranial skeleton, the greatest number of changes (more than five) are seen in

573 the transition from the subadult to the young adult growth categories (Fig. 10) that include, in

574 descending order, the pes, fibula, scapula, coracoid, and humerus. Thereafter, postcranial

575 changes effectively cease until three clusters of changes in adulthood, at the $14^{\text {th }}$ and $15^{\text {th }}$ stages,

576 the $18^{\text {th }}$ and $19^{\text {th }}$ stages, and a final set at the $21^{\text {st }}$ growth stage.

577 Early changes (juvenile to young adult categories) happen to the pectoral girdle and pes

578 ( $6^{\text {th }}$ and $7^{\text {th }}$ growth stages $)$, the humerus ( $6^{\text {th }}$ growth stage $)$, and the fibula ( $8^{\text {th }}$ growth stage).

579 Later changes (adult to senescent adult categories) are seen in the axial column $\left(15^{\text {th }}, 19^{\text {th }}\right.$, and

$58021^{\text {st }}$ growth stages $)$, pelvic girdle $\left(14^{\text {th }}\right.$ and $18^{\text {th }}$ growth stages $)$, and the fibula $\left(18^{\text {th }}\right.$ and $19^{\text {th }}$

581 growth stages). This pattern suggests a relatively instantaneous transition in the pectoral girdle

582 and limb, and the pes; in contrast, changes to the axial column, and to the pelvic girdle and limb,

583 are relatively sustained throughout growth.

584

585 Craniomandibular modules. Recent work has found that the skull of T. rex is organized into

586 six modules of functional integration (Werneburg et al., 2019). The growth series obtained here

587 shows that the modules experience different amounts of ontogenetic change (Fig. 11; Table 4): in

588 descending order, the dorsum of the snout, circumorbital bones, and frontals; the sides of the 
589 snout and palate; the mandibular ramus; the braincase; the suspensorium; and the parietal bone.

590 The modular pattern of the skull is considered evidence for a flexible framework (this hypothesis

591 conflicts with inferences based on Finite Element Analysis (FEA) modeling; Cost et al., 2019);

592 in terms of growth, the suspensorium, parietal, and braincase are the modules that change the

593 least (less than 10 changes per growth stage), which is consistent with their stable keystone-like

594 function against which the relatively flexible palate and facial skeleton can passively move and

595 the lower jaws freely rotate.

596 The greatest change across modules (more than 10) occurs in the $5^{\text {th }}$ and $6^{\text {th }}$ growth

597 stages; this corresponds to the transition from small to large juveniles (Fig. 11). The transition

598 from the long and low skulls of juveniles to the tall skulls of subadults imposes 52 changes,

599 where the snout dorsum module is changed from the delicate and thin morphotype of juveniles to

600 the greatly expanded and inflated form of subadults.

601 Changes to the skull roof and mandible are seen throughout ontogeny; changes to the

602 snout and supensorium occur later, from the $5^{\text {th }}$ growth stage onwards. Other modules do not

603 change until young adulthood, including the parietal and braincase; changes to the parietal are

604 limited to young adults and adults, whereas changes to the braincase continue to the last growth

605 stage. These differences in the timing of change to modules is consistent with the hypothesis of

606 modularity, otherwise they would change together if they were integrated. During the interval of

607 greatest change, in the transition from juveniles to subadults (growth stages 5 and 6), the

608 modules that change the most are the skull roof, snout, and mandible.

609

610 Ontogram mapped onto growth curve. Mapping the ontogram onto a previously published

611 growth curve for T. rex (Erickson et al., 2004) permits refinement of the diagnosis of the higher- 
612 level growth categories of Carr (1999) and Erickson et al. (2004), namely juvenile, subadult,

613 young adult, adult, and senescent adult (Fig. 12). This approach provides a framework for

614 comparison with previous studies on functional morphology in T. rex and the evolution of its

615 ontogeny. This template serves as a heuristic device and is not intended to estimate growth rates;

616 for example, the position of BMRP 2006.4.4 on the steepest part of the growth curve conflicts

617 with the histological evidence that the individual was at a moderate growth rate before death

618 (Woodward et al., 2020). At best, the curve is an approximation of the true growth rate. The

619 chronological age of BMRP 2002.4.1 is based on the revised age of Woodward et al. (2020); the

620 age of LACM 23845 (14 years; Erickson et al., 2004) is considered here to be an underestimate

621 since it conflicts with that of the less mature BMRP 2006.4.4 (15 years; Woodward et al., 2020).

622

623 Growth Categories

624 Alignment between the growth series, chronological age, cortical histology, number of

625 growth changes, growth rate, and, to a lesser degree, size and mass, were used to define five

626 growth categories, once the ontogram was mapped onto the growth curve of Erickson et al.

627 (2004), a procedure that was constrained by seven histologically aged specimens (Fig. 12).

628 Although mass is a relevant variable in these comparisons, given the variety of methods used by

629 different workers, the published mass estimates conflict with each other (Table 5); for that

630 reason, the decision for each mention of mass as a relevant diagnostic character is given below.

631 Also, although the masses are given to the nearest kilogram or, in four instances, to the tenth of a

632 kilogram, it is not intended to give the impression of precision, rather the exact estimates are here

633 simply reproduced unaltered from the original sources. To reflect the natural imprecision, mass 
634 estimates given in the next section are rounded up in the discussion of diagnostic features of 635 growth categories.

636

637 Juveniles

638 Juveniles (=adolescent category of Erickson et al., 2004) have a skull length less than 80 $639 \mathrm{~cm}$, long and low skulls with a length to height ratio of 3.0 or greater, are equal to or less than 13 640 years old, and lack an EFS. This growth category sees 58 unambiguously optimized growth 641 changes, of which 24 have a CI of 1.0 (Table 6). In the skull, most changes are seen in the snout 642 module (sensu Werneburg et al., 2019). This category corresponds to growth stages 1 to 5 of the 643 ontogram (Fig. 2) and to the initial lag phase of the T. rex growth curve of Erickson et al. (2004; 644 Fig. 12). The juvenile stage extends from hatching to the $13^{\text {th }}$ year of life, before the long and 645 low skull proportions are lost at the subadult category.

646 A single mass estimate of $39.9 \mathrm{~kg}$ has been published for the small juvenile LACM 64728471 by Erickson et al. (2004). Two mass estimates have been published for the large juvenile 648 BMRP 2002.4.1: a range of 639 to $1,269 \mathrm{~kg}$, and a mean of $954 \mathrm{~kg}$ was published by Hutchinson 649 et al. (2011) and an estimate of $660.2 \mathrm{~kg}$ was published by Snively et al. (2019). The maximum 650 estimate is used here as a point of reference; therefore, juveniles are here considered to have a 651 mass equal to or less than $\sim 1,300 \mathrm{~kg}$.

652 The juvenile growth stage is diagnosed by the possession of a dorsoventrally shallow 653 skull and jaws, the increase in height of which defines the subadult stage. To precisely reflect the 654 primary changes that occur even during this initial phase of life, this growth category is divided 655 into two categories in the following diagnosis, namely small juveniles and large juveniles. A 656 total of five growth changes occur in the small juvenile growth stage, which affect the skull roof 
657 and mandible modules of Werneburg et al. (2019). In contrast, 53 changes occur in the large

658 juvenile growth stage that are more widespread across the skull, including modules of the skull

659 roof, snout, suspensorium, and mandible. The large juvenile category marks the earliest onset of

660 sexual maturity (see below).

661

662 Small Juveniles

663 Joint surfaces. In the fourth growth stage, there is a clear distinction between the postorbital

664 buttress and the caudal shelf of the frontal bone.

665 Neurovasculature. In the second growth stage, the alveolar row of foramina that penetrates the 666 lateral surface of the dentary acquires a sulcus that unites all of the openings. In medial view, the 667 Meckelian sulcus becomes a deeply inset groove.

668 Dorsotemporal fossa. In the fourth growth stage, the sagittal crest of the frontal becomes tall 669 and the fossa extends rostrodorsally at a steep angle relative to the level forehead (subcutaneous 670 surface ahead of the fossa).

671

672 Large Juveniles

673 Skull frame. In the fifth growth stage, several correlates of an increase in skull height are seen, 674 including the dorsal position of the angulation in the rostral margin of the premaxilla, the 675 rostrodorsal orientation of the nasal process of the premaxilla, an oval orbital fenestra, vertical 676 orientation of the postorbital bar with the presence of a depression and strut on the lateral surface 677 of the postorbital process of the jugal, the inset and braced ventral end of the jugal process of the 678 postorbital, and presence of the subocular process of the postorbital. 
680 (Henderson, 2002; Bates and Falkingham, 2012; Bates and Falkingham, 2018; Gignac and

681 Erickson, 2017; Cost et al., 2019) and their corresponding high loads upon the skull and teeth.

682 These include a convex cross section of the rostral third of the nasal and its frontal ramus, the tall

683 height of the antorbital fossa below the internal antorbital fenestra, the straight rostral margin of

684 the rostroventral ala of the lacrimal, and the concave dorsal margin of the vomer.

685 Other changes are almost certainly unrelated to the increase in skull height, including the

686 straight dorsal margin of the bony naris, the presence of a contribution of the jugal to the margin

687 of the internal antorbital fenestra, enhancement of the plane change between the temporal region

688 and the orbitosnout region, the medioventral orientation of the lateral surface of the maxilla to

689 the alveolar margin of the bone, the intermediate position of the bony choana, the rostralward

690 shift of the ventral margin of the joint surface for the quadratojugal ramus on the jugal, the

691 straight shape of the dentary in ventral view, and caudalward position of the shallowest point of

692 the dentary.

693 Joint surfaces. In the fifth growth stage an alteration for a stable skull frame is seen, namely a

694 distinct maxillary flange that produces a deep slot for the nasal. The plane change between snout 695 and orbitotemporal region is reflected in the sinuous jugopostorbital suture.

696 Subcutaneous surface. In the fifth growth stage, an increase in the relief of the subcutaneous

697 surface of the face is seen, where alveolar skirts are present at the premaxilla, the body of the

698 jugal is rugose, indicating the appearance of armor-like skin (Carr et al., 2017), and subcutaneous

699 rugosities extend onto the medial surface of the postorbital. 
700 Cephalic ornamentation. In the fifth growth stage, some changes in cephalic ornamentation are

701 independent of the texture of the subcutaneous surface, including the decrease in size of the

702 cornual process of the jugal and the increase in the number of midline bumps on the nasals.

703 Paranasal pneumaticity. In the fifth growth stage, internal inflation of the maxillary sinus is

704 seen externally, shown by the convex rostrodorsal surface of the bone. Some changes to the

705 antorbital fossa might correspond to internal changes of the dentition, including elimination of

706 the rostral end of the crease that defines the ventral margin of the external antorbital fenestra.

707 In the maxilla, the rostroventral corner of the external antorbital fossa is indistinct, but it

708 is difficult to determine whether this is caused by a difference in pneumaticity or of the

709 subcutaneous surface; the antorbital fossa is deeply inset relative to the lateral surface of the

710 bone, especially rostrodorsally; and the rostral pneumatic recess of the palatine becomes longer

711 than the caudal recess.

712 Neurovasculature. In the fifth growth stage, the number of foramina rows in the nasal increases

713 to three and the rostrodorsal foramen of the tract of the subnarial foramen makes its appearance.

714 Adductor musculature. In the fifth growth stage, the insertion for the ventral pterygoid muscle

715 on the surangular and dentary is enhanced, the dorsal margin of the postorbital is everted

716 medially, and in rostral and medial views its dorsal margin is convex.

717 Several bony changes almost certainly reflect an increase in the forcefulness of the

718 adductor musculature, including rostrally converging dorsal and ventral margins of the caudal

719 ramus of the prearticular. Changes in the adductor musculature impose alterations to bones that

720 are not directly related to increased bite force; for example, the rostral ramus of the prearticular

721 is paddle-shaped, which in life medially increased the bony enclosure around the inserted

722 musculature. 
723 Joint capsules. In the fifth growth stage, the horizontal ridge on the ventral quadrate process of

724 the quadratojugal is absent, and the lateral scar below the glenoid of the surangular becomes

725 rugose.

726 Dentition. In the fifth growth stage, the maxillary tooth count increases to 16 and the dentary

727 tooth count increases to 17; and the first maxillary tooth becomes subincisiform. The teeth and

728 antorbital fossa complex are useful points of reference for capturing changes in tooth size. For

729 example, in the fifth growth stage less than six teeth are ahead of the external antorbital fossa,

730 which almost certainly reflects enlargement of the teeth if, and only if, the rostral margin of the

731 fenestra is a stable landmark throughout ontogeny. Geometric morphometric quantification of

732 skull shape in T. bataar shows that the rostral end of the fossa shifts caudally during growth

733 (Foth et al., 2016). If the same trend occurs in T. rex, and if there was no increase in the size of

734 teeth then an increase in the number of teeth ahead of the fossa would be seen. Therefore, tooth

735 size increases despite the caudalward shift of the fossa.

736

737 Subadults

738

Subadults (= juvenile category of Erickson et al., 2004) have a skull length from $80 \mathrm{~cm}$ to

739

$1.1 \mathrm{~m}$, tall skulls with a length to height ratio less than 3.0 (deduced from the straight form of the

740 ventral ramus of the lacrimal in LACM 23845 and RSM 2990.1, in contrast to the strongly

741 curved form seen in juveniles such as CMNH 7541 and BMRP 2002.4.1), are from 15 to 17

742 years old, and lack an EFS. The maximum number of changes in the entire growth series, 106,

743 are seen in this growth category; 69 of the synontomorphies have a CI of 1.0 (Table 7). In the

744 skull the changes are limited to the skull roof module. The maximum number of postcranial

745 changes, 48 , occur in this growth stage and affect the limb girdles and appendages. This category 
746 corresponds to growth stages 6 and 7 of the ontogram (Fig. 2) and to the first half of the

747 exponential phase of the T. rex growth curve (Erickson et al., 2004; Fig. 12). The presence of

748 medullary bone in BMRP 2006.6.4 (Woodward et al., 2020) marks the latest onset of sexual

749 maturity (Fig. 12).

750 A mass estimate of $1,807 \mathrm{~kg}$ was published for LACM 23845 by Erickson et al. (2004);

751 no other estimates have been published for this specimen. Therefore, a range of mass greater

752 than $\sim 1,300 \mathrm{~kg}$ and equal to or less than $\sim 1,810 \mathrm{~kg}$ (the mass seen in young adults) is considered

753 here as diagnostic of subadults.

754 The transition from the long and low skull and narrow teeth of juveniles to the tall and

755 powerful skull and thick teeth of subadults occurred shortly before the halfway point of life

756 (assuming a lifespan of at least 28 years; Erickson et al., 2004), within a narrow two-year

757 interval between the growth categories, at the start of the exponential phase of growth (sensu

758 Erickson et al., 2004). This rapid change to the skull frame and dentition marks the onset of the

759 increase in bite force; an order-of-magnitude increase in bite force between juvenile and adult $T$.

760 rex was found by Bates and Falkingham (2012). This increase is almost certainly congruent with

761 the niche partitioning that is hypothesized to ecologically separate juveniles from adults (Snively

762 et al. 2006). Therefore, this extreme transition in skull shape and function occurs before the onset

763 of somatic maturity (i.e., adult size; sensu Erickson et al., 2004) (Fig. 12). Also, the presence of

764 medullary bone in BMRP 2006.6.4 shows that T. rex is typically reptilian given that sexual

765 maturity precedes somatic maturity (Erickson et al., 2007; Lee and Werning, 2008).

766 Skull architecture. In the sixth growth stage, several changes to the lacrimal reflect an increase

767 in bite force, including the 7-shaped bone, a rostrocaudally long ventral ramus, a dorsoventrally

768 deep rostrodorsal process, a long rostroventral process that separates the nasal from the maxilla, 
769 a sliver-like maxillary process, the medially extended and convex medial margin of its dorsal

770 ramus, the lengthened region caudal to the lacrimal pneumatic recess, and abruptly

771 rostroventrally extending rostrodorsal margin of the rostral ramus.

772 Other architectural changes are seen in the lacrimal that are less obviously the result of

773 high loads: the distal (=ventral) end of the orbitonasal ridge is mediolaterally wide but tapers

774 ventrally; the ridge is not backswept; the orbitonasal ridge grades into the ventral ramus ahead of

775 it; the caudal edge of the ridge is positioned far ahead of the caudal margin of the ventral ramus;

776 the ridge extends abruptly rostroventrally dorsally, above the rostroventral ala; the rostrodorsal

777 margin of the ventral ramus is straight or concave; and the rostroventral margin of the ventral

778 ramus is straight. In the seventh growth stage, the rostroventral margin of ventral ramus of the

779 lacrimal is concave.

780 Joint surfaces. In the sixth growth stage, several changes to the lacrimal are seen, including the

781 joint surface for the prefrontal on the dorsal ramus of the lacrimal is a dorsoventrally deep and

782 laterally shallow groove that caudally twists to face mediodorsally, the joint surface for the nasal

783 on the rostral ramus covers its ventral third, the dorsal half of the medial joint surfaces is

784 dorsoventrally shallow, the medial joint surface (behind the medial tube) is concave in vertical

785 section, a groove on the dorsal surface of the frontal process is present, and a medially extending

786 process behind the joint surface for the prefrontal is present.

787 Subcutaneous surface. In the sixth growth stage, the subcutaneous surface of the lacrimal wraps

788 onto the caudal surface of the ventral ramus and the coarse patch caudodorsal to the lacrimal

789 pneumatic recess is absent.

790 Cephalic ornamentation. In the sixth growth stage, the low ridge caudodorsal to the lacrimal

791 pneumatic recess is lost. 
792 Paranasal pneumaticity. In the sixth growth stage, the invasive antorbital air sac produces the

793 medial pneumatic recess of the lacrimal. Some pneumatic changes are almost certainly an

794 epiphenomenon of the overall dorsoventral and mediolateral expansion of the skull, including the

795 distal pneumatic recess of the lacrimal that is positioned close to maxilla. Some pneumatic

796 changes more directly reflect the resorptive tendency of pneumatic epithelia, including the

797 absorption of the caudoventral margin of the medial pneumatic recess.

798 Inflation of the dorsal ramus of the lacrimal has several simultaneous effects upon the

799 bone, including obscuring the apex of the cornual process and the process in general; increase in

800 the height of the cornual process; loss of the ridge that rostrally bounds the conchal surface;

801 increase of the depth of the ridge above the conchal surface; the convex caudodorsal region of

802 the shallow conchal surface; the convex lateral surface around the lacrimal pneumatic recess; the

803 laterally bulging region of the cornual process of the lacrimal; increase in the height of the

804 subcutaneous surface above the antorbital fossa to twice the height of the rostral ramus of the

805 lacrimal; reduction of the size of the lacrimal pneumatic recess to less than half the length of the

806 region behind it; inflation of the region below the lacrimal pneumatic recess, and the septum

807 ahead of it, which merges the junction of the antorbital fossa and subcutaneous surface ahead of

808 the lacrimal pneumatic recess; the shallowly concave region between the distal recess and the

809 septum ahead of the lacrimal pneumatic recess; the lengthened septum between the accessory

810 recesses; the shallowly concave distal pneumatic recess of the lacrimal; the widened supraorbital

811 process and reduction of its length and increase in its height; and the caudal end of the snout is

812 widened by the inflation of the lacrimals. 
In the seventh growth stage, inflation has obliterated the cornual process of the lacrimal.

814 A single accessory recess, the distal recess, is present ahead of the lacrimal pneumatic recess,

815 which is reduced to a foramen.

816 Neurovasculature. In the sixth growth stage, the foramen in the ridge that bounds the lacrimal

817 pneumatic recess is absent, and the dorsal margin of the medial tube of the lacrimal extends

818 rostrodorsally.

819 Appendicular skeleton. In the seventh growth stage several changes are seen in the pectoral

820 girdle and limb. In the scapula, the acromial region is most deeply concave on the scapula, the

821 glenoid fossa has an intermediate position between lateral and ventral, the glenoid fossa is

822 narrower than the shaft, the anterolateral corner of glenoid widens toward the coracoid, the

823 medial surface of the acromion is convex, the muscle scar along the dorsal edge of the shaft is

824 indistinct, and the ridge on lateral surface of the shaft is absent. The coracoid also shows

825 changes, including a posteroventral orientation of the glenoid, an abrupt separation of the glenoid

826 from the coracoid process, loss of the ridge that extends anteriorly from the biceps tubercle, the

827 ventral half of the bone is convex, and the medial fossa ahead of the coracoid foramen stops

828 short of the anterior edge of the bone. Finally, the deltoid scar at the distal end of the

829 deltopectoral crest of the humerus is deeply inset.

830 In the seventh growth stage, the lip of the glenoid of the scapula extends from the shaft at

831 an abrupt angle, the shaft is not distinctly narrow between the acromial region and the shaft, and

832 the ventral surface of the shaft next to the glenoid faces ventrally. Changes in the coracoid

833 include the anterolateral orientation of the coracoid foramen and the stout form of the coracoid

834 process. 
836 margin of the proximal joint surface of D II PH 2 is concave, the anterior end of the lateral distal

837 condyle is flattened, the medial distal condyle extends anterodorsally, the proximodorsal flange

838 is wide, the medial distal condyle extends anterolaterally, and it extends posteriorly below the

839 level of the posterior margin of the medial collateral ligament pit. In D III PH 1, the proximal

840 joint surface is dissected by sulci and the lateral collateral ligament pit is lens-shaped. In D IV

$841 \mathrm{PH} \mathrm{1,} \mathrm{the} \mathrm{ventromedial} \mathrm{condyle} \mathrm{extends} \mathrm{at} \mathrm{a} \mathrm{steep} \mathrm{angle} \mathrm{relative} \mathrm{to} \mathrm{the} \mathrm{shaft,} \mathrm{the} \mathrm{ventral} \mathrm{notch} \mathrm{of}$

842 the proximal joint surface is positioned at the midline, the distal joint surface is much wider than

843 tall, the scar on the lateral surface of the shaft ahead of the proximal end is distinct, the

844 dorsolateral surface of the shaft is convex, and the ridge lateral to the supracondylar pit is short.

845 In D IV PH 3, the phalanx is short, the proximal joint surface is distinctly wider than tall, the

846 lateral collateral ligament pit is deep, and the flexor muscle scar is coarse. In D IV PH 4, the

847 dorsum of the bone is wide, the mediolateral orientation of the dorsum is distinct, the proximal

848 end of the bone is wider than the distal end, the distal joint surface is distinctly concave, the

849 entire posterior margin of the distal condylar region is undercut by a groove that separates it from

850 the ventral surface of the shaft, and the indentation along the dorsal margin of the distal joint 851 surface is wide.

852 In the seventh growth stage, changes are seen in D IV PH 1, where the supracondylar pit 853 is a deep crease that sharply elevates the distal condyles. In D IV PH 3, supracondylar pit is

854 deep, the medial margin of the proximal joint surface is distinctly convex, the medial collateral

855 ligament pit is large, and the distal condyles shallowly diverge from each other. 856

\section{Young Adults}


$859 \mathrm{~m}$, tall skulls with a length to height ratio of 2.6 to 2.3, are from 18 to 22 years old, lack an EFS, 860 and, as seen in subadults, they initially have a high number of synontomorphies, which thereafter

861 drops in frequency (Figs. 2, 4). In total, this growth stage has 77 growth changes; 29 have a CI of

8621.0 (Table 8 ). In the skull, the greatest number of changes is seen in the skull roof and braincase

863 modules. In contrast to subadults, growth changes are seen across every module of the skull.

864 Postcranial changes are limited to the hindlimb. This category corresponds to growth stages 8 to

86512 of the ontogram (Fig. 2), occurring in the last half of the exponential phase of the T. rex

866 growth curve (Erickson et al., 2004; Fig. 12).

867 Mass estimates for the young adult TMP 1981.006.0001 range from 3,230 kg (Erickson

868 et al., 2004) to 4,469 kg (Persons et al., 2019). Another young adult, MOR 1125, has been

869 estimated at $6,100 \mathrm{~kg}$ (Persons et al., 2019). This doubling in mass from 1,810 $\mathrm{kg}$ subadults to

$8703,000 \mathrm{~kg}$ young adults occurred halfway through life, in the three-year interval (15 to 18 years) in

871 the middle of the exponential phase of growth (Erickson et al., 2004); this ontogenetic increase

872 in mass corresponds to the phylogenetic threshold in mass, where T. rex exceeds the 3,000 $\mathrm{kg}$

873 limit of its closest relatives, namely Daspletosaurus torosus and T. bataar (Snively et al., 2019).

874 Therefore, this character state in T. rex is hypermorphic in that it suprasses the ancestral

875 condition (cf. Erickson et al., 2004).

876 Skull architecture. In the seventh growth stage, the rostral margin of the rostroventral lamina of

877 the lacrimal is convex, the jugal ramus of the postorbital is rostrocaudally wide and parallel-

878 sided, the apposed frontals are wider than long, the caudal end of the joint surface for the

879 prefrontal on the frontal is positioned below the joint surface for the postorbital, the orbital

880 surface of the frontal is horizontally oriented, the midline strut of the nuchal crest that is above 
881 the supraoccipital is prominent, the ventral bar of the dentary that is below the Meckelian groove

882 is dorsoventrally deep, and the secondary ridge on the medial surface of the angular is distinct.

883 In the eighth growth stage, the dorsal and ventral margins of the ascending ramus of the

884 maxilla converge as they extend caudally, the point of rostralmost incursion of the internal

885 antorbital fenestra is positioned within less than five teeth of the caudal end of the tooth row, the

886 subocular process of the postorbital is as long or longer than the frontal process, and the chin of

887 the dentary is positioned ahead of the fourth alveolus.

888 In the ninth growth stage, the caudal end of the antorbital fossa has a uniform height

889 below the internal antorbital fenestra and it is obliterated (i.e., fades out) ahead of the jugal

890 ramus, and the lateral margin of the premaxilla is straight or convex. In the tenth growth stage,

891 the concavity in the caudal surface of the nuchal crest fades below the dorsal margin of the crest,

892 and the ventral surface of the neck of the occipital condyle has the form of a deep pit between

893 pillars. In the eleventh growth stage, the dorsal surface of the neck of the occipital condyle is

894 penetrated by a shallow pit.

895 Joint surfaces. In the seventh growth stage, the orbital notch in the frontal bone is pinched

896 between the joint surfaces for the lacrimal and postorbital; the dorsal joint surface for the

897 quadrate of the quadratojugal is wide and coarse, its rostral margin is concave, and its caudal half

898 is developed into a wide ridge; the frontoparietal junction is wider than the sagittal crest rostral

899 and caudal to it; and the joint surface for the dentary on the splenial is reinforced by a wide

900 longitudinal ridge.

901 In the ninth growth stage, the joint surface for the quadratojugal on the jugal is positioned

902 far caudal to the cornual process. In the tenth growth stage, the joint surface for the squamosal on

903 the postorbital is coarsened by deep and numerous longitudinal ridges, the caudal shelf of the 
904 postorbital extends subtly ventrolaterally, and a ridge extends along the laterosphenoidoprootic

905 suture. In the eleventh growth stage, the rostral margin of the parietal extends mediolaterally

906 (i.e., it is not wedge-shaped), the postorbital buttress extends caudal to the midlength of the

907 dorsotemporal fossa, and the lateral extent of the parietofrontal suture extends from

908 mediolaterally to caudolaterally.

909 Subcutaneous surface. In the seventh growth stage, the subcutaneous surface of the ventral

910 ramus of the lacrimal is coarse with papillae concentrated toward its rostral margin. In the eighth

911 growth stage, lateral fossae are present on the alveolar process of the maxilla. In the ninth growth

912 stage, the circumfossa ridge of the maxilla is low or absent, and the texture of the subcutaneous

913 region of the chin is highly rugose. In the tenth growth stage, the groove that extends across the

914 prefrontolacrimal process is deeply incised.

915 Cephalic ornamentation. In the ninth growth stage, the cornual process of the postorbital

916 exceeds the height of the bone.

917 Paranasal pneumaticity. In the eighth growth stage, the maxillary fenestra closely approaches

918 the ventral margin of the external antorbital fenestra, the interfenestral strut is rostrocaudally

919 narrow, the caudodorsal margin of the maxillary fenestra is straight or concave, the ventral

920 margin of the maxillary fenestra is obliterated and incorporated into the antorbital fossa, and the

921 mediolateral depth of the antorbital fossa is deep. In the ninth growth stage, the dorsal surface of

922 the lacrimal extends dorsomedially, and the ventral edge of the subcutaneous surface at the

923 septum ahead of the lacrimal pneumatic recess extends rostroventrally or grades into the

924 antorbital fossa without a distinct orientation.

925 Endoccipital pneumaticity. In the eighth growth stage, the caudal surface of the paroccipital

926 process is deeply concave and the caudodorsal surface is strongly convex. In the ninth growth 
927 stage, the bar that extends across the level of the lateral processes of the supraoccipital is

928 prominent.

929 Basicranial pneumaticity. In the eighth growth stage, the subcondylar fossa is obliterated by

930 inflation. In the ninth growth stage, the pneumatic foramina of the subcondylar fossa are

931 positioned far apart from each other (cf. Witmer and Ridgley, 2009). In the eleventh growth

932 stage, the rim of the caudoventral end of the subsellar recess is spout-like, and the ventral end of

933 the preotic pendant is a low ridge.

934 Dorsotemporal fossa. In the seventh growth stage, the sagittal crest of the frontal is equal to or

935 greater than $37 \%$ the length of the bone, and the sagittal foramen is positioned far caudal to the

936 rostral margin of the fossa (indicating rostralward encroachment of the musculature).

937 Muscle scars. In the seventh growth stage, the ridge along the squamosal process of the

938 quadratojugal is mediolaterally wide in rostral view, and the nuchal crest is rugose but the texture

939 does not reach the midline of the crest. In the eighth growth stage, the lateral half of the nuchal

940 surface of the squamosal is coarsely textured, and the oval scar of the basisphenoid is oriented

941 medioventrally. In the ninth growth stage, the oval scar faces lateroventrally and it is coarse in

942 texture.

943 In the tenth growth stage, the rugose texture of the nuchal crest of the parietal reaches the

944 midline of the crest, the ventral end of the metotic strut is scoured by the oval scar complex, and

945 the basal tuber is coarse in texture. In the eleventh growth stage, the pits mediodorsal to the

946 supraoccipital extend deeply into the nuchal crest, the dorsal rugosities of the nuchal crest extend

947 onto the rostral surface of the crest, and the ventrolateral edge of the base of the rostrolateral

948 process of the parietal is crossed by a subtle, ventrolaterally extending ridge. 
949 Dentition. In the eighth growth stage, the first mesial alveolus of the dentary is substantially

950 smaller than the alveoli at the middle of the tooth row (i.e., the second alveolus is enlarged), and

951 the number of dentary alveoli is 13 .

952 Appendicular skeleton. In the seventh growth stage, the fibula is stout, the proximal end of the

953 bone is differentiated into distinct anterior and posterior knob-like convexities that produces a

954 deeply concave dorsal margin, the anterior surface of the fibula distinctly widens toward its

955 distal (=ventral) end, the medial fossa of the fibula is sharply differentiated into anterior and

956 posterior fossae, the posterior extent of the medial fossa is a wide ridge that separates the fossa

957 from the posterior edge of the bone, and the anterior surface of the bone above the iliofibularis

958 tubercle (= bipartite scar) is wide and flat before becoming dorsally a narrow but blunt ridge.

959

960 Adults

$961 \quad$ Adults (=young adult of Erickson et al., 2004) have a skull length of 1.3 to $1.4 \mathrm{~m}$, a skull

962 length to height ratio of 2.6 to 2.3 , are greater than 22 years old, have an EFS, and, as is seen in

963 subadults and young adults, initially have a high number of growth changes, which rapidly

964 drops. Sixty-four synontomorphies occur in this category, and 13 of them have a CI of 1.0 (Table

965 9). In the skull, the greatest number of changes, 15 and 13, occurs in the snout and skull roof

966 modules, respectively, and changes are seen in all modules (Table 4). Eight changes occur in the

967 postcranium, where five occur in the pelvic girdle and limb, and three are seen in the axial

968 skeleton. The adult stage corresponds to growth stages 13 to 20 of the ontogram (Fig. 2) and to

969 the first part of the stationary phase of the T. rex growth curve (Erickson et al., 2004; Fig. 12).

970 An ontogenetic progression among adults, based on size, was first hypothesized by Paul,

971 "[AMNH FARB 50270 and other big T. rex specimens may or may not be subadults. This is 
972 possible because the biggest specimen (UCMP 118742]...is 29 percent longer than 5027" (1988:

973 344-345). Indeed, the results here recover this exact hypothesis of relative maturity (Figs. 1, 2).

974 Several mass estimates are available for adults (Table 5). The mass for adults is 10,200

$975 \mathrm{~kg}$ in Henderson and Snively (2004), 6,071.8 kg in Bates et al. (2009), 5,777 kg to 10,768 kg in

976 Hutchinson et al. (2011), 4,660 kg to 10,007 kg in Campione et al. (2014), 6,986.6 kg in Snively

977 et al. (2019), and 5,112 to 8,870 kg in Persons et al. (2019). The minimum estimated masses of

978 adults are greater than 4,500 kg (Hutchinson et al., 2011; Campione et al., 2014; Persons et al.,

979 2019), which overlaps with that of young adults (Table 5). In contrast, the mean mass estimates

980 for adults exceed those for young adults, indicating that a mass greater than 5,100 $\mathrm{kg}$ might be

981 diagnostic for adults, whereas the range of mass in young adults is from $\sim 3,000 \mathrm{~kg}$ to $\sim 5,100 \mathrm{~kg}$.

982 Given the variety of techniques used for estimating mass in T. rex, the differences in the results

983 between them, and the incomplete sampling of specimens, mass is here only tentatively

984 considered informative in distinguishing between the young adult and adult categories.

985 Skull architecture. In the thirteenth growth stage, the lateral depression of the jugal is shallow

986 and enhanced by ridges, the surangular shelf extends lateroventrally, the angulation in the ventral

987 margin of the maxilla is distinct, the ventral jugal process of the maxilla is a massive convex

988 strut, the rostrodorsal margin of the rostral ramus of the lacrimal extends sharply rostroventrally,

989 the rostral end of the medial maxillary process of the jugal above the joint surface for the maxilla

990 is sharply inset caudally, the base of the medial process of the squamosal is separated by a notch

991 from the margin of the dorsotemporal fenestra, the caudodorsomedial edge of the shaft of the

992 quadrate is distinctly concave, the rostral margin of the vomeropterygoid process of the palatine

993 is positioned ahead of the rostral palatine recess, and the lateral margin of the basisphenoid is

994 deeply embayed. 
In the fourteenth growth stage, the narrow caudal end of the ascending ramus of the

996

997

998

999

1000

1001

1002

1003

1004

1005

1006

1007

1008

1009

1010

1011

1012

1013

1014

1015

1016

1017

maxilla above the internal antorbital fenestra twists along its course, the tip of the rostrodorsal

process of the lacrimal extends rostrally past the midlength of the internal antorbital fenestra, the lateral depression of the postorbital process of the jugal reaches the level of the rostral end of the postorbital contact, the parietal is elaborated into a ridge above each of the tines of the dorsal process of the supraoccipital, the Meckelian groove of the dentary is positioned at the midheight of the bone rostrally and above the midheight caudally, and the caudal margin of the dentary is deeply concave.

In the fifteenth growth stage, the convex dorsal margin of the dentary extends to alveolus 7. In the seventeenth growth stage, the dorsal margin of the postorbital is swollen such that the medial eversion is completely obscured. In the eighteenth growth stage, the medial edge of the caudal margin of the quadratojugal is positioned caudal to the lateral edge of the bone. In the nineteenth growth stage, the rostralmost incursion of the internal antorbital fenestra is at the level of the fifth tooth from the caudal end of the tooth row, the rostroventral margin of the ventral ramus of the lacrimal is straight, the lateral ridge of the ventral postorbital process of the squamosal extends more ventrally than laterally, the dorsal margin of the jugal ramus of the quadratojugal extends to the medial edge of the shaft, and the ventral margin of the ramus if ventrally convex.

In the twentieth growth stage, the quadrate fossa of the quadratojugal is shallow in depth, the ventral ramus of the lacrimal below the medial pneumatic recess is convex in vertical section, the nasal ramus of the frontal is wide and truncated, a ventrally-extending flange is absent from the ventral margin of the basioccipital, and the Meckelian groove is positioned below the midheight of the dentary rostrally and above midheight caudally. 
1018 Joint surfaces. In the thirteenth growth stage, the dorsolateral surface of the ventral

1019 quadratojugal process of the jugal is dominated by a single large ridge, the dorsal margin of the

1020 joint surface for the quadrate of the ventral quadrate process of the quadratojugal extends

1021 rostrodorsally, the joint surface for the pterygoid on the caudal process of the ectopterygoid is

1022 deeply excavated, and the joint surface for the splenial on the ventral bar of the dentary has a

1023 peg-in-socket form.

1024 In the fourteenth growth stage, the condylar surface of the occipital condyle has a distinct

1025 marginal rim. In the sixteenth growth stage, the joint surface for the splenial on the lingual bar of

1026 the dentary is inset. In the eighteenth growth stage, the concavity in the ventral margin of the

1027 quadratojugal process of the squamosal is absent, and the joint surface for the otoccipital on the

1028 squamosal is convex.

1029 In the nineteenth growth stage, the distal end of the jugal ramus of the postorbital is

1030 wedge-shaped to fit into a complementary groove in the jugal, the joint surface for the medial

1031 frontal process of the nasal is long and reaches caudally past the rostral end of the joint surface

1032 for the prefrontal, the rostral margin of the Meckelian fossa extends ventrolaterally to the medial

1033 surface of the bone, and the prearticuloangular buttress is present. In the twentieth growth stage,

1034 the joint surface for the palatine is present medially and rostrally on the rostroventral ala of the

1035 lacrimal.

1036 Paranasal pneumaticity. In the thirteenth growth stage, the distal recess of the rostral ramus of

1037 the lacrimal faces more ventrally than laterally. In the nineteenth growth stage, a strut extends

1038 across the medial wall of the secondary fossa of the jugal.

1039 Basicranial pneumaticity. In the twentieth growth stage, the subcondylar foramen of the

1040 basioccipital emits a deeply inset channel from its lower margin. 
1041 Neurovasculature. In the thirteenth growth stage, the medial surface of the ventral ramus of the

1042 lacrimal is scoured by deep sulci. In the fourteenth growth stage, the subnarial foramen produces

1043 a deep notch in the rostral margin of the maxilla, and lateromedially penetrating foramina in the

1044 antorbital fossa of the maxilla are present. In the eighteenth growth stage, the caudal surface of

1045 the jugal process of the ectopterygoid is not perforated by a foramen, and lateromedially

1046 penetrating foramina are absent from the antorbital fossa of the maxilla.

1047 Dorsotemporal fossa. In the thirteenth growth stage, the rostral margin of the dorsotemporal

1048 fossa of the frontal is elaborated into a ridge or crest.

1049 Subcutaneous surface. In the thirteenth growth stage, the texture of the caudal process of the

1050 squamosal is coarsely rugose.

1051 Cephalic ornamentation. In the nineteenth growth stage, the epipostorbital is fused to the

1052 underlying bone.

1053 Dentition. In the thirteenth growth stage, the number of dentary teeth is 14 . In the seventeenth

1054 growth stage, the rostral margins of the interdental plates are notched (almost certainly an

1055 epiphenomenon of tooth enlargement).

1056 Axial skeleton. In the fifteenth growth stage, the joint surface of the axial intercentrum is

1057 dorsoventrally deep, and the spinous process of the axis is dorsoventrally massive. In the

1058 nineteenth growth stage, the hypophysis is present on the cervical centra.

1059 Appendicular skeleton. In the fourteenth growth stage, the ischium has two semicircular scars.

1060 In the eighteenth growth stage, the ventral margin of the public peduncle of the ilium extends

1061 horizontally and the anterior surface of the fibula below the bipartite scar is narrow. In the

1062 nineteenth growth stage, the distal end of the shaft of the fibula flares abruptly to the ventral joint 
1063 surface that produces a deep crease in the shaft, and the anterior margin of the distal end of the

1064 fibula bulges anteriorly.

1065

1066 Senescent Adults

1067 Finally, senescent adults have a skull length of at least $1.4 \mathrm{~m}$, are at least 28 years old,

1068 and have an EFS. In the skull, the greatest number of changes occurs in the skull roof module,

1069 and the rest of the synontomorphies are limited to the snout, suspensorium, and the braincase

1070 modules, and postcranial changes occur in the axis (Table 4). Of the 19 synontomorphies that

1071 support this growth stage, only one has a CI of 1.0 (Table 9). This category corresponds to

1072 growth stage 21 of the ontogram (Fig. 2) and to the end of the stationary phase of the T. rex

1073 growth curve (Erickson et al., 2004; Fig. 12); there is a single exemplar specimen, FMNH

1074 PR2081.

1075 The estimated mass for FMNH PR208 is greater than those obtained for other adults in

1076 Hutchinson et al. (2011) and Snively et al. (2019) (Table 5). However, its mass is exceeded by an

1077 adult in the comprehensive samples of Campione et al. (2014) and Persons et al. (2019);

1078 therefore, the mass of senescent adults does not exceed the range seen in adults and so it cannot

1079 be used to diagnose this growth stage.

1080 Skull architecture. The antorbital fossa below the internal antorbital fenestra is extremely

1081 shallow, low ridges extend into the quadrate cotyle of the squamosal, and the orbital notch is a

1082 deep cleft in the orbital surface of the frontal.

1083 Joint surfaces. The joint surface for the palatine on the rostroventral ala of the lacrimal is

1084 present medially only, the crease between the postorbital buttress and shelf of the frontal is 
1085 present, and the joint surface for the prefrontal in the orbital surface of the frontal is positioned

1086 close to the orbital notch.

1087 Subcutaneous surface. The dorsolateral surface of the lacrimal is rugose.

1088 Cephalic ornamentation. The cornual process of the postorbital does not interrupt the

1089 rostrolateral corner of the dorsotemporal fossa.

1090 Paranasal pneumaticity. The caudalmost extent of the trough medial to the tooth root bulges is

1091 limited to the maxillary antrum.

1092 Neurovasculature. The medial surface of the ventral ramus of the lacrimal is smooth, the

1093 foramen that penetrates the orbital surface of the jugal ramus of the postorbital is far medial to

1094 the lateral margin of the bone, and the ventral surface of the basituberal web is not penetrated by

1095 a foramen.

1096 Muscle scars. The suborbital ligament scar is convex or bulbous, the lateral surface between the

1097 jugal process and ventral quadrate process of the quadratojugal is scoured by a deep fossa that is

1098 secured by a ridge, and the mediolaterally extending ridge ahead of the parietofrontal suture is

1099 absent.

1100 Axial skeleton. The anterior surface of the axial intercentrum is deeply concave and bounded

1101 ventrally by a prominent rim, a deep groove separates the ventral edge of the anterior joint

1102 surface of the intercentrum from the ventral surface of the bone, the anterior margin of the

1103 intercentrum is concave and the posterior margin is convex, and the concave ventral surface of

1104 the intercentrum is limited to the ventral and lateroventral surfaces of the bone by the swollen

1105 parapophysis.

1106

1107 Craniomandibular variation (Molnar, 1990) 
1108 The results obtained here provide an opportunity to review the variation reported by

1109 Molnar (1990) in his extensive descriptive monograph of the skull and mandibular rami of $T$.

1110 rex, and recast in the context of ontogeny.

1111 Facial skeleton. The foramen in the base of the antorbital fossa of the maxilla in LACM 23844

1112 is individual variation. The difference in shape of the maxillary fenestra seen between adults is

1113 almost certainly an artifact of variability in the form of its caudodorsal margin, ventral margin,

1114 and rostral margin. Comparison of the shapes of the fenestra (trapezoidal, oval, triangular)

1115 described by Molnar (1990) with the ontogram do not show a sequential pattern. The absence of

1116 "discernable sculpture" on the maxilla of TMP 1981.006.0001 (Molnar, 1990: 142), taken here

1117 to mean the absence of fossae on the horizontal ramus, represents individual variation.

1118 The difference in the angle and position of the flexure of the jugal ramus of the maxilla

1119 might correspond to relative maturity, where it changes from rostral and distinct (e.g., AMNH

1120 FARB 5027, SDSM 12047) to caudal and indistinct (e.g., LACM 23844). Likewise, the change

1121 from a large third maxillary antrum chamber (e.g., LACM 23844; Molnar, 1990: Fig. 2) to a

1122 large second chamber (e.g., UCMP 118742) corresponds to an increase in relative maturity. The

1123 foramen in the caudodorsal surface of the interfenestral strut in CM 9380 is individual variation.

1124 The dorsoventral position of the palatal process of the maxilla is broadly congruent with relative

1125 maturity, where it is ventral in position in relatively immature adults (e.g., AMNH FARB 5027,

1126 SDSM 12047) and dorsal in relatively mature adults (UCMP 118742). However, these

1127 conditions are seen in specimens that share growth stage 17 (ventral in CM 9380, dorsal in

1128 LACM 23844). This character was not included in the character matrix; it may turn out to

1129 separate the specimens at growth stage 17 from each other, if, and only if, the character states 
1130 can be verified and the analysis re-run with them included. The absence of interdental pits from

1131 the maxilla are individual variation unique to SDSM 12047.

1132 Although Molnar (1990) regarded absence of sculpture on the subcutaneous surface of

1133 the maxilla, lacrimal, and nasal in TMP 1981.006.0001 as evidence for immatuirty, based on the

1134 results here it is individual variation, as well as the coarse texture of the nasal that is seen in

1135 MOR 008. The difference between flattened premaxillary processes of the nasal in AMNH

1136 FARB 5027 and the rod-like processes in LACM 23844 and MOR 008 are consistent with

1137 increasing relative maturity.

1138 Molnar's (1990) comments on the relative development of the cornual process of the

1139 postorbital deserve some comment. The process is a composite structure that includes a rugosity

1140 that is an intrinsic part of the bone to which an overlying osteoderm, termed here the

1141 epipostorbital, is attached. The epipostorbital is sometimes difficult to distinguish from the

1142 underlying postorbital, but it is clearly distinguished by its distinctly flat ventral surface along

1143 which a distinct, tuberculate, and eave-like flange extends (Molnar, 1990). The epipostorbital is

1144 not preserved in LACM 23844, which accounts for its difference from the other specimens. The

1145 progression in development regarded by Molnar (1990), from SDSM 12047 to AMNH FARB

11465027 to MOR 008, does follow the progression of maturity found here. However, the reduced

1147 condition of the epipostorbital among the most mature specimens (growth stages 19, 20),

1148 indicates a reversal of the pattern. A growth-related reduction in ornamentation is seen in other

1149 dinosaurs (e.g., Triceratops, Pachycephalosaurus; Horner and Goodwin, 2006; Horner and

1150 Goodwin, 2009).

1151 Palate and quadrate. The rostral process of the vomer is large in the less mature AMNH FARB

1152 5027, whereas it is small in the more mature LACM 23844. The pits seen in the caudal process 
1153 of LACM 23844 are regarded here as damage or individual variation, since they are not seen in

1154 any other T. rex specimens (AMNH FARB 5027, BMRP 2002.4.1, MOR 008, UWBM 99000).

1155 The foramen in the ventral surface of the palatine in AMNH FARB 5027 is regarded here as

1156 either individual variation or a lesion, since this feature is not seen in any other specimen of $T$.

1157 rex. The difference in texture of the caudal process (=anteroventral part of the pterygoid limb

1158 (Molnar, 1990)) of the ectopterygoid is not congruent with the growth series found here, which

1159 follows a sequence from SDSM 12047 (not rough) to LACM 23844 (rough) to MOR 008 (not

1160 rough). The thickness of the quadrate process of the pterygoid is congruent with the growth

1161 series, which is thick in the subadult category (LACM 23845), whereas it is thin in adults (e.g.,

1162 LACM 23844, MOR 008, SDSM 12047). A possible ontogenetic progression is seen on the

1163 medial surface of the orbital process of the quadrate, which is coarse in the less mature SDSM

1164 12047, whereas it is smooth in the more mature LACM 23844.

1165 Braincase. Molnar (1990) reported that the joint surface for the laterosphenoid on the postorbital

1166 is variable, where it is convex (AMNH FARB 5117), shallowly concave (TMP 1981.012.0001),

1167 or deeply concave (LACM 23844). However, examination of AMNH FARB 5117 for this study

1168 found that the joint surface is concave. It is possible that the difference between shallow and

1169 deep states is ontogenetically informative, but the rim that surrounds the joint surface is often

1170 damaged and missing, preventing a precise assessment of depth.

1171 Mandibular ramus. Molnar suggested that the intercoronoid is fused to the dentary in most

1172 specimens of T. rex, but that condition has not been seen during the course of this study; in all

1173 cases, the bones are separate. Molnar (1990) is correct in stating that the right angular of MOR

1174008 is coarsened by lesions, and so this is not a growth-related change. The fusion of the

1175 prearticular reported by Molnar (1990) in MOR 008 might be a growth-related co-ossification 
1176 event. The thickened surangular shelf seen in LACM 23844, in contrast to the thinner condition

1177 reported in AMNH FARB 5027 (Molnar, 1990), might be another growth-related change.

1178 Molnar (1990) noted the presence of perforations in the lateral plate of the surangular in

1179 T. rex; these purportedly pathological features (Wolff et al., 2009) deserve comment. Three

1180 perforations occur in the same location in different specimens. The first penetrates the bone

1181 rostroventral to the caudal surangular foramen (csf) at the dorsal margin of the angular; this is

1182 seen in two young adults (MOR 1125, UWBM 99000) and in one adult (MOR 980). The second

1183 penetrates the region ahead of the csf below the surangular shelf; this is seen in one young adult

1184 (UWBM 99000), three adults (MOR 008, MOR 980, RSM 2523.8), and in the senescent adult

1185 (FMNH PR2081). The third penetrates the rostroventral quadrant of the bone ahead of the

1186 external mandibular fenestra, which is seen in one young adult (UWBM 99000), an adult (MOR

1187 008), and in the senescent adult (FMNH PR2081). Given the spotty distribution of these

1188 openings, and, indeed, their lesion-like appearance, they were not included in the cladistic

1189 analysis of growth; regardless, the regularity of their positions indicates that they might not be

1190 lesions.

1191 Suture closure. Molnar documented numerous cranial (postorbitojugal, quadratoquadratojugal,

1192 squamosoquadrate) and mandibular (angulosurangular, prearticulosurangular) suture closures in

1193 the adult MOR 008, which he regarded as ontogenetic in nature. Given the relatively immature

1194 maturity of the specimen, and the fact that closure of these sutures are not seen in other T. rex,

1195 and they are regarded here as individual variation; in at least one case, a clear lesion is seen at the

1196 point of fusion (e.g., right angulosurangular joint). Also, it is not obvious that the right

1197 quadratoquadratojugal suture is closed in the specimen. 
1198 Ontogenetic variation among adults. Molnar (1990) regarded the texture of the subcutaneous

1199 surface of facial bones, inflation of cranial sinuses, joint fusions, development of the cornual

1200 process of the postorbital, as reflecting relative maturity. Also, he proposed that the variable

1201 shape of the maxillary fenestra and the joint surface for the laterosphenoid of the postorbital

1202 represent individual variation. The results here broadly agree with these assessments. He was

1203 less certain of the significance of the form of the premaxillary process of the nasal, difference in

1204 height of the palatal process of the maxilla and the form of the rostral plate of the vomer

1205 (Molnar, 1990). The present study does not resolve the latter set of features, but they will be

1206 taken up in a future iteration of this work.

1207

1208 Secondary Metamorphosis

1209 The abrupt, two-year transition in T. rex from the sleek craniomandibular skeleton of

1210 juveniles to the deep and stout form of subadults (a change that is terminal) is an example of

1211 secondary metamorphosis, an extreme transformation of morphology that is associated with

1212 sexual maturity (Rose and Reiss, 1993). Every bone and anatomical domain (e.g., paranasal air

1213 sac system, dentition, skull frame, musculature, integumentary system, etc.) is involved in

1214 reshaping the entire head skeleton, making juveniles and mature specimens so different that they

1215 have been taken to be different taxa (e.g., Molnar, 1980; Bakker et al., 1988). The occurrence of

1216 multiple transformative events across the head skeleton (i.e., not a single event) is the primary

1217 evidence for metamorphosis (Rose and Reiss, 1993). This case of secondary metamorphosis

1218 coincides with the trophic shift from juvenile to subadult, and, almost certainly, with sexual

1219 maturity, whereas it precedes somatic maturity (Erickson et al., 2004). This hypothesis can be

1220 rejected if, upon discovery of relatively complete large juvenile and subadult specimens, a 
1221 quantitative comparison of bite force between large juveniles and subadults does not show a

1222 discontinuous increase in the magnitude of bite force between the growth categories.

1223

1224 Bite Force and Maturity

1225 Different methods have been used to estimate bite forces of T. rex, including adults (e.g.,

1226 Erickson et al., 1996; Gignac and Erickson, 2017) and a juvenile (Bates and Falkingham, 2012;

1227 Table 10). In each case, the maximum estimated bite force of the juvenile is consistently an order

1228 of magnitude lower than those for adults (Table 10). The only exception to this is the allometric-

1229 scaling based estimate of Meers (2002) for adult T. rex, which is an order of magnitude lower

1230 than the other estimates of maximum bite force that were obtained by other methods; regardless,

1231 the minimum estimate $(7,600 \mathrm{~N}$; Meers, 2002) is greater than that of the maximum bite force of

1232 the juvenile (3,850 N; Bates and Falkingham, 2012).

1233 Like crocodylians (Gignac and O'Brien, 2016), T. rex has a high bite force for its body

1234 size (Bates and Falkingham, 2012). Bite force estimates for juvenile T. rex (2,600 to 4,010 N;

1235 BMRP 2002.4.1) are lower than those of adults (35,000 to 57,000 N) and show a growth trend of

1236 positive allometry, when identical methods of bite force estimation have been used for both

1237 growth categories (Bates and Falkingham, 2012); i.e., bite forces in adults are relatively and

1238 absolutely higher than those seen in juveniles.

1239 In light of these data, a comparison of bite force was made here between a sample of

1240 several adult T. rex (Gignac and Erickson, 2017) and a juvenile (Bates and Falkingham, 2012).

1241 Given that different methods were used to estimate bite force in the juvenile, on the one hand,

1242 and its adult counterparts on the other, the raw estimates were converted into ranks for a

1243 Spearman rank correlation test of the congruence between maturity and bite force (Table 10). 
1244 The data were tested for normality and a Shapiro-Wilk test; the growth ranks and bite force ranks

1245 are normally distributed ( $p=0.961$ for both). The Spearman rank correlation test resulted in a

1246 significant $(p=0.042)$ correlation coefficient $\left(r_{s}=0.829\right)$. Therefore, an ontogenetic progression

1247 of increasing bite force from juvenile to adult, and throughout adulthood (Fig. 13), is seen in $T$.

1248 rex, which reflects the condition that is reported in crocodylians (Gignac and Erickson, 2014).

1249 Tooth cross sectional shape serves as a proxy for bite force, where the tooth width to

1250 length ratio in crocodylians continuously increases with the ontogenetic increase in bite force

1251 (Gignac and Erickson, 2014). In T. rex, the tooth width to length ratio doubles from juveniles

1252 (e.g., BMRP 2002.4.1, maxillary tooth 4, width to length ratio: 54\%) to adults (e.g., MOR 008,

1253 maxillary tooth 4, width to length ratio: 98\%), which might be evidence for a delay in the

1254 necessity for indenting and fracturing the bones of prey in juveniles, in contrast to the continuous

1255 pattern that is seen in crocodylians (Bates and Falkingham, 2012; Gignac and Erickson, 2014).

1256 Ergo, a complete sample of bite force estimates across the metamorphic transition in T. rex is

1257 required to test this hypothesis, which is based on gross morphology.

1258 The relationship between bite force and size is linear in extant crocodylians, despite the

1259 great changes in the size and type of prey species, a clade that, unlike $T$. rex, does not undergo an

1260 abrupt, wholesale ontogenetic transformation in skull shape (Erickson et al., 2003, 2013). If bite

1261 forces in T. rex do increase abruptly across metamorphosis, then this is fundamentally unlike the

1262 situation in crocodylians, where bite force matches or exceeds prey shear forces in crocodylians

1263 (Gignac and Erickson, 2014). In contrast, apical tooth pressures in the dinosaur will be found to

1264 not equal or exceed the maximum shear strength of significantly larger prey until metamorphosis

1265 has occurred. If true, then the ontogenetic change reflects a discontinuous and abrupt dietary 
1266 change to significantly larger prey that was necessary to sustain the metabolic demands of a 1.8-

1267 tonne animal on its way to becoming a $3+$ tonne predator.

1268 Tooth morphology. A comparison of tooth width to length ratios gives some indication of

1269 whether or not an abrupt, discontinuous increase in bite force is reasonable to expect in T. rex

1270 growth. The comparisons made here are based on the lateral teeth of the maxilla and dentary; i.e.,

1271 excluding the incisiform first two teeth of the maxilla and the two mesial subconical teeth of the

1272 dentary that have higher width to length ratios than the successive ziphiform teeth. In large

1273 juveniles (BMRP 2002.4.1, $n=34$ ) the mean width to length ratio across all teeth is $54 \%$; in

1274 young adults (MOR $1125, n=20$ ) the ratio is $68 \%$, and in adults (MOR 008, $n=14$; LACM

$127523844, n=6$; MOR 980, $n=2$; $\operatorname{RSM} 2523.8, n=10$ ) the ratio is $79 \%, 71 \%, 85 \%$, and $85 \%$,

1276 respectively (Table 11).

1277 Bite force estimates for the juvenile are 2,400 $\mathrm{N}$ for the mesial teeth and 3,850 $\mathrm{N}$ for the

1278 distal teeth (Bates and Falkingham, 2018); the minimum bite force estimates for a subadult

1279 (TMP 1981.006.0001) is 12,197 N, and in adults (MOR 008, LACM 23944, MOR 980) the

1280 estimates are 13,736 N, 16,352 N, and 14,201 N, respectively (Gignac and Erickson, 2017).

1281 Therefore, the order-of-magnitude increase in bite force, and the $12 \%$ increase in tooth width that

1282 is seen between juveniles and subadults, are naively predicted here to occur abruptly in the

1283 subadult stage, no later than 15 years old.

1284

\section{Tooth Count and Maturity}

1286 In T. rex, tooth count in the maxilla and dentary initially increases, and then decreases

1287 over the course of the growth series. Despite this general trend, variation is seen from the young

1288 adult growth stage onwards; therefore, the congruence between maturity and the tooth number in 
1289 the maxilla and the dentary was tested using Spearman rank correlation, assuming a null

1290 hypothesis of noncorrelation.

1291 Correlation with maxillary tooth count. The bivariate scatterplot of ranked data (Fig. 14, Table

1292 12) shows that maxillary tooth number initially increases between the first two growth stage

1293 ranks (from 15 to 16 teeth) before it abruptly decreases (from 16 to 12 teeth) at the third rank

1294 (Fig. 14). Tooth count rank is constant until the sixth rank, where variation, toward a lower tooth

1295 count (from 12 to 11 teeth), is first seen (Fig. 14). Prima facie, tooth loss covaries with maturity,

1296 as is seen in other tyrannosaurids (Carr et al., 2017).

1297 A Shapiro-Wilk test found that the maxillary tooth rank data are not normally distributed

$1298(p=0.004)$, whereas the growth rank data are normally distributed $(p=0.725)$; a Spearman rank

1299 correlation test of growth rank and tooth count ranks was done, which resulted in a statistically

1300 nonsignificant $(p=0.073)$ correlation coefficient $\left(r_{S}=0.494\right)$. Therefore, a general trend of

1301 decrease in tooth count is not seen across the entire data set. This result must be regarded with

1302 the caveat that there is a gap in the data, where maxillary tooth counts are currently unknown for

1303 subadult specimens. It is possible that tooth counts from future discoveries of subadults will

1304 bridge the gap between the extremes and result in a significant value.

1305 In addition to that, the hypothesis that tooth count is a reliable proxy for maturity from

1306 young adults to senescent adults was tested by limiting the statistical test to the corresponding

1307 portion of the data set (growth stage ranks 3 to 14); a statistically nonsignificant result was

1308 obtained $\left(r_{S}=0.112, p=0.729\right)$, indicating that the null hypothesis of no difference could not be

1309 rejected. Ergo, maxillary tooth count is an unreliable proxy for maturity among adult-sized

1310 specimens. 
1311 Correlation with dentary tooth count. As for the maxilla, an initial increase (from 16 to 17)

1312 followed by a decrease in tooth count (from 17 to 14 ) is seen in the dentary, after which variation

1313 is seen (from 14 to 12) among adults (Fig. 15). The raw data were converted to ranks (Table 13),

1314 and a Shapiro-Wilk test of normality found that the dentary tooth count rank data are not

1315 normally distributed $(p=0.021)$, whereas the growth rank data are normally distributed $(p=$

1316 0.616); a Spearman correlation test of the data resulted a significant $(p=0.005)$ correlation $\left(r_{S}=\right.$

1317 0.648), indicating a trend of decrease in tooth count across the data set. Among adults, the

1318 hypothesis of dentary tooth count as a proxy for maturity was tested by obtaining the correlation

1319 coefficient from young adults to senescent adults (from growth rank 3 to 17), which resulted in a

1320 nonsignificant $(p=0.074)$ correlation $\left(r_{S}=0.475\right)$, indicating that dentary tooth count is not a

1321 reliable proxy for estimating maturity among adult-sized animals.

1322

1323 Congruence between Chronological Age, Size, and Mass with Maturity

1324 Congruence between age, size, and mass (dependent variables) with maturity

1325 (independent variable) was tested using Spearman rank correlation. As used here, maturity refers

1326 to position of stages along the ontogram. The chronological age of BMRP 2006.6.4. is

1327 incongruent with maturity, where its chronological age of 15 years is greater than the more

1328 mature LACM 23845 that is estimated as 14 years old (Erickson et al., 2004). Given this

1329 discrepancy, the age of LACM 23845 is regarded here as an underestimate.

1330 Correlation with age. A bivariate scatterplot shows congruence of ranked data between growth

1331 rank and age rank (Fig. 16, Table 14); a Shaprio-Wilk test found that the chronological age ranks

1332 and growth ranks are normally distributed $(p=0.885$ and 0.933 , respectively). A significant $(p=$

$13330.000)$ correlation $\left(r_{S}=0.970\right)$ was obtained between age and maturity, indicating that LAG 
1334 counts are an accurate predictor of maturity. The $r_{S}$ of less than 1.0 is caused by the

1335 chronological age of one specimen (LACM 23845) that is almost certainly an underestimate (see

1336 above), and two specimens (MOR 1125, TMP 1981.006.0001) that have the same LAG count of

1337 18. Therefore, chronological age can be used as a proxy for relative maturity throughout the

1338 growth series as long as sampling from a skeleton is sufficiently thorough (cf. Woodward et al.,

1339 2020).

1340 Correlation with size. The scatterplot between maturity and size of ranked data shows an

1341 overall increasing trend, but with variation among the adult specimens (Fig. 17, Table 15). A

1342 Shapiro-Wilk test of normality found that the ranked size data and growth rank data are normally

1343 distributed ( $p=0.166$ and 0.678 , respectively). The Spearman rank correlation test resulted in a

1344 significant $(p=0.000)$ correlation $\left(r_{S}=0.903\right)$, showing that size can serve as a proxy for

1345 maturity; however, this congruence is seen among animals that are less than adult size. A second

1346 test was run on the animals in the adult size range (growth ranks 5 to 15), where a significant ( $p$

$1347=0.008)$ correlation $\left(r_{\mathrm{S}}=0.748\right)$ was obtained, indicating that among adult-sized animals, size is

1348 a reliable proxy for maturity. Even with MOR 1125, a relatively small young adult (femur

1349 length: $1.16 \mathrm{~m}$ ), excluded (limiting the comparison to growth ranks 6 to 15 ), the results are

1350 significant $\left(r_{\mathrm{S}}=0.661, p=0.038\right)$. However, when the test is limited to large adults (skull length

1351 greater than $1.2 \mathrm{~m}$; growth ranks 8 to 15$)$ the results are nonsignificant $\left(r_{\mathrm{S}}=0.312, p=0.451\right)$,

1352 indicating that size cannot serve as a proxy for maturity among the largest adults.

1353 Correlation with mass. The scatterplot between congruence between maturity and mass of

1354 ranked data (Fig. 18, Table 16) shows a cloud of points with an increasing trend, indicating

1355 correlation. A Shapiro-Wilk test found that the ranked mass data and growth rank data are

1356 normally distributed ( $p=0.914$ and 0.870 , respectively). The Spearman correlation rank test 
1357 resulted in a significant $(p=0.019)$ correlation $\left(r_{S}=0.753\right)$ between the variables; a second run

1358 of the test was made for the adult-sized animals (growth stage ranks 3 to 9) since this region of

1359 the distribution shows variation. The test resulted in a nonsignificant $(p=0.289)$ correlation $\left(r_{S}=\right.$

1360 0.468), indicating that mass is a poor predictor of maturity among the adult growth categories.

1361

\section{Congruence between Nonbiological Factors and Maturity}

1363 The possibility that the growth series obtained here results from the influence of abiotic

1364 factors, namely geographic location and stratigraphic position, was tested using the Spearman

1365 rank correlation test since horizontal and vertical spatial data are hierarchical in time and space.

1366 For example, the growth series might reflect the north to south distribution of a latitudinally

1367 variable population, the anagenetic transformation of a population, or both trends. To test these

1368 hypotheses, geographic location was ranked by latitude along a north-south gradient that follows

1369 the long axis of Laramidia (Table 17, Fig. 19), and stratigraphic position was ranked according to

1370 position within the lower, middle, and upper units of the Hell Creek Formation (HCF; Table 18,

1371 Fig. 20). To protect specimen localities and prevent trespassing, the precise locality coordinates

1372 are not provided here; locality data are available, to qualified researchers, from the museums

1373 where specimens are accessioned. Data for stratigraphic position was obtained from the literature

1374 (Fowler, 2017; Gates et al., 2019; Harrison et al., 2013; Horner et al., 2011; Leslie et al., 2018),

1375 aside from the specimens (DDM 344.1) whose positions were obtained from museum records.

1376 Correlation with geographic position. A Shapiro-Wilk test found that the ranked location data

1377 and growth rank data are normally distributed ( $p=0.277$ and 0.228 , respectively). The

1378 geographic location test of ranked data (Table 17) resulted in a statistically nonsignificant $(p=$

$13790.219)$ correlation $\left(r_{\mathrm{S}}=-0.236\right)$, indicating that the location of the specimens along the growth 
1380 series was not influenced by geographic location. This result is robust given that it was possible

1381 to code a geographic location for nearly every specimen in the growth series (Fig. 19). The

1382 correlation between unranked data (UTM coordinate northings) and growth stage was tested

1383 using a Pearson correlation test; data for 17 specimens were available for this test and the data

1384 are skewed to the right. The test resulted in a nonsignificant $(p=0.588)$ correlation coefficient $(\mathrm{r}$

$1385=-0.141)$, which is consistent with the test on the ranked data.

1386 Correlation with stratigraphic level. The stratigraphic schema that is presented here for late

1387 Maastrichtian formations from western North America is largely based on Fowler (2017), where

1388 the threefold stratigraphic division of the HCF of Montana is used as the basis of comparison

1389 with other units. As such, the comparison spans the last million years of the late Maastrichtian,

1390 which is divided into three chronostratigraphic slices, in descending order: 398 Kya (equivalent

1391 to chron 29r), $286 \mathrm{Kya}$, and $\sim 286 \mathrm{Kya}$ (Fowler, 2017). Using this approach, the Frenchman

1392 Formation of Saskatchewan is equivalent to the upper HCF (Fowler, 2017). The radiometric

1393 dates and stratigraphic revisions of Leslie et al. (2018) were followed to correlate the Tornillo

1394 Formation of Texas with the upper HCF. Given the imprecise dating of the Scollard and Willow

1395 Creek Formations of Alberta, and ambiguous correlation between them and the upper and middle

1396 members of the HCF, specimens from those units were excluded. This comparison was made

1397 aware of the fact that the stratigraphic position of specimens might be affected by time

1398 transgression and so might not be equivalent. Resolving that issue is beyond the scope of this

1399 work, the results of which are offered here as a hypothesis for further, more rigorous testing of

1400 stratigraphic correlation.

1401 A Shapiro-Wilk test found that the ranked stratigraphic data are not normally distributed

$1402(p=0.030)$, whereas the growth rank data are normally distributed $(p=0.933)$. A Spearman rank 
1403 correlation test was run, which recovered a nonsignificant $(p=0.654)$ correlation $\left(r_{\mathrm{S}}=0.189\right)$

1404 between stratigraphic position and maturity (Fig. 20, Table 18). However, this result is not robust

1405 given that only eight specimens are included in the sample and only two are from the middle

1406 member of the HCF (BMRP 2002.4.1; Harrison et al., 2013; DDM 344.1) (Fig. 20). Therefore,

1407 more specimen data are required for a rigorous test of the noncorrelation between maturity and

1408 stratigraphic position. Regardless, the scatterplot shows that a young adult, two adults, and

1409 senescent adult have been collected from the lower member of the HCF, two juveniles from the

1410 middle unit, and one subadult and two adults from the upper member; i.e., broadly overlapping

1411 growth series have been recovered throughout the unit.

1412

1413 Dimorphism Hypothesis (sensu Carpenter, 1990)

1414 Carpenter (1990) identified two sets of characters in the skull and postcranium of T. rex

1415 that he considered to represent patternless variation (noise; i.e., individual variation) on the one

1416 hand, and patterned variation (signal; i.e., sexual variation) on the other. Noise included: maxilla

1417 depth, size of the maxillary fenestra, shape of the maxillary fenestra, size of the antorbital

1418 fenestra, shape of the antorbital fenestra, position of the lacrimal process of the maxilla, position

1419 of the jugal process of the maxilla, the shape of the jugal process of the maxilla, and shape of the

1420 dentary. In contrast, signal included: robust and gracile morphs of cervical vertebrae and angle of

1421 the ischium from the caudal series. The cladistic results obtained here agree that, among adults,

1422 the depth of the maxilla and size and shape of the maxillary and antorbital fenestrae, the position

1423 and shape of the lacrimal and jugal processes of the maxilla, and shape of the dentary, are noise.

1424 Also, Carpenter (1990) found that the maxilla TMM 41436-1 was an outlier in contrast to

1425 the sample of adult maxillae, which were scaled to the rostral end of the maxilla and the rostral 
1426 end of the maxillary fenestra. The incomplete and small TMM 41436-1 was scaled to the height

1427 of the maxillary fenestra (Carpenter, 1990), which is proportionately taller than in adults (e.g.,

1428 AMNH FARB 5027). The comparison showed that it is different from the others in that the

1429 rostral margin of the bone falls short of the adults, which was used by Carpenter (1990) to argue

1430 the specimen represents a new taxon; however, based on the results found here, this difference

1431 almost certainly arises from the fact its teeth are not as enlarged in adults and the internal sinuses

1432 are not expanded, changes that greatly reshape the bone in adults. Therefore, the difference in

1433 shape is most simply explained by maturity; when included in the cladistic analysis of ontogeny,

1434 the specimen falls out in a polytomy that includes subadult, young adult, and adult specimens

1435 (Fig. 1B).

1436 Carpenter (1990) found, qualitatively, that the cervical series of AMNH FARB 5027 was

1437 less massive than that of BM R7994. In particular, Carpenter (1990) drew attention to the form

1438 of the atlantal intercentrum and that of the spinous process of the axis and the third cervical

1439 vertebra, characters that were included in the cladistic analysis presented here (characters 1210,

1440 1256, and 1280, respectively; see Data S1). The results found that those specimens are

1441 ontogenetically sequential, where AMNH FARB 5027 is less mature than BM R7994, indicating

1442 that the differences (slender to massive) are ontogenetic. It is worth pointing out that the

1443 illustrations that were used to show the differences between the specimens are mislabeled, where

1444 the massive BM R7994 is mistakenly labeled as the slender AMNH FARB 5027, and vice versa

1445 (Carpenter, 1990: Fig. 10.4 A, B).

1446 Finally, Carpenter (1990) compared the several features of the ischium between three

1447 specimens of T. rex. He identified the orientation of the ischia as informative, where in two (CM

1448 9380, TMP 1981.006.0001) the bone extends sharply posteroventrally whereas the third (AMNH 
1449 FARB 5027) extends at a lower angle. Carpenter (1990) hypothesized that the steep condition

1450 was required for passing eggs in life, and the bearers were female, whereas the bearer of the

1451 other form was male. The results of the cladistic analysis here found that of these three

1452 specimens, TMP 1981.006.0001 is the least mature and that CM 9380 is the most mature.

1453 Therefore, a growth sequence from steep (TMP 1981.006.0001) to less steep (AMNH FARB

1454 5027), and back to steep (CM 9380) is seen. If the angles of divergence are real, then a lack of

1455 signal (noise) is probably the best explanation for the observation.

1456 However, the position of the tip of the ischium is an important landmark for this

1457 comparison, but it is missing from CM 9380 (Osborn, 1906: fig. 7) whereas it is shown as

1458 complete in Carpenter (1990: fig. 10.5). The complete condition presumably reflects restoration

1459 of the bone as indicated by the presence of a small boot at its tip (Carpenter, 1990: fig. 10.5), a

1460 structure that is not seen in complete specimens of T. rex or in tyrannosaurids in general. Also,

1461 the shaft of the ischium in CM 9380 is intermediate in position between the two other specimens

1462 in that it overlaps the dorsal margin of the ischium of TMP 1981.006.0001 (Carpenter, 1990: fig.

1463 10.5). Given these issues, the character was included a posteriori in the cladistic data matrix and

1464 only the three specimens mentioned by Carpenter (1990) were coded in the analysis, using the

1465 state assignments given by him. The analysis recovered one 3,054-step ontogram and the

1466 topology is unaffected. Therefore, based on this sample, ischial divergence does not group

1467 specimens together, indicating that it is not dimorphic; regardless, a larger data set for this

1468 character is required for a rigorous test of whether the two states are valid to begin with.

1469

1470 Sexual Dimorphism and "Tyrannosaurus " ${ }^{69}$ " (sensu Larson, 2008) 
A topological pattern of sexual dimorphism was not recovered in the ontogram, which is

1472 linear and pectinate (Fig. 2). If dimorphism was present in the data, which covers the entire

1473 skeleton, and if the sample included examples of each sex, then a branch of females that includes

1474 BMRP 2006.6.1 and MOR 1125, the only purportedly unambiguous females in the sample,

1475 should be united by the presence of shared skeletal sexual correlates, aside from femoral

1476 medullary bone (Schweitzer et al., 2005; Woodward et al., 2020). The failure to recover a female

1477 branch indicates that such correlates are absent from T. rex. Neither did a comparison of

1478 individual variation recover a dimorphic pattern (see above). The alternative explanation for

1479 these results is that BMRP 2006.6.4 and MOR 1125 are the only females in the sample, but this

1480 is improbable given the high number of adult-grade specimens in the analysis.

1481 Regardless, several specimens included in this study have been hypothesized as male

1482 (TMP 1981.006.0001, MOR 008, MOR 555) or female (MOR 1125, RSM 2523.8, CM 9380,

1483 FMNH PR2081) and so an account of the difference between those results (Larson, 2008) and

1484 those obtained here is required. No grouping pattern is seen when male ("gracile") and female

1485 ("robust") morphs (sensu Larson, 2008) are mapped onto the ontogram, where both "morphs"

1486 are interspersed among the adult specimens (Fig. 21). A biologically meaningful signal would be

1487 indicated if, for example, gracile morphs occurred earlier in growth than the robust morphs, or

1488 vice versa. Given that both morphs are seen in young adult and adult growth stages, the simplest

1489 explanation is that they do not represent a developmental pattern aside from individual variation

1490 upon the attainment of the asymptote of size.

1491 Several lines of evidence were offered to support the inference of a binary difference

1492 between male and female morphs (Larson, 2008). However, it was found here that those

1493 purported dimorphic features actually show a continuum of variation (e.g., circumference to 
1494 length ratio of the humerus, height to length ratio of the ilium, circumference to length ratio of

1495 metatarsal II), whereas others show no pattern (e.g., ratio of third to second dentary teeth,

1496 divergence of the ischium, circumference to length ratio of the femur) (Fig. 22).

1497 Larson (2008) referred three specimens (AMNH FARB 5027, MOR 008, SDSM 12047)

1498 to the taxon "Tyrannosaurus " $x$ "”. If this taxon was valid, then the specimens should be

1499 recovered sharing a single branch, separate from the other adults. However, the specimens are

1500 scattered throughout the adult growth category of the ontogram, indicating that the characters

1501 thought to unite them (size of lacrimal pneumatic foramen, maxillary and dentary tooth count,

1502 form of second dentary tooth, ratio of the length of the third to second dentary tooth) actually

1503 reflect ontogenetic or individual variation, not taxonomically informative characters (Fig. 22).

1504 Finally, a comparison of individual variation (Data S5) was done to identify any shared

1505 characters that might provide evidence of common identity. Individual variation was recovered

1506 for AMNH FARB 5027 and SDSM 12047, but not for MOR 008. In addition to that, there are no

1507 shared characters between the two former specimens; therefore, no character evidence was found

1508 that supports the purported taxon.

1509

1510 Dentary Groove

1511 It has been claimed by Schmerge and Rothschild (2016a) that the neurovascular groove

1512 along the alveolar row of foramina of the dentary in tyrannosaurids has a binary expression of

1513 present and absent. Among latest Cretaceous tyrannosaurids of Laramidia, they hypothesized

1514 that the groove is absent from T. rex, regardless of size, whereas it is present in "Nanotyrannus

1515 lancensis", which otherwise has been shown to be an invalid taxon (Carr and Williamson, 2004). 
1517 the dentary groove were re-examined for this study and it was found that in two of them the

1518 groove is present, but greatly reduced in extent (AMNH FARB 5027, MOR 008). In addition to

1519 that, a reduced groove is seen in all adult T. rex, including the type specimen (CM 9380, LACM

1520 23844, MOR 555, MOR 980, MOR 1125, UWBM 99000) (Data S1). These grooves are true

1521 sulci, and are not artifacts of foramen shape, and so are not "pseudo-grooves" (sensu Schmerge

1522 and Rothschild, 2016b).

1523 Although the states of this character were not unambiguously optimized on the ontogram,

1524 the specimens show a stepwise progression: the groove is absent from the least mature specimen

1525 (LACM 28471), the groove is present along the entire row in more mature juveniles (e.g., BMRP

1526 2002.4.1), and, finally, the groove is reduced to short segments in adults (e.g., FMNH PR2081)

1527 and so cannot be said to be absent from them. The groove is almost certainly obscured in adults

1528 by the overall expansion of the bone in response to growth, higher loads imposed by ontogenetic

1529 increase in body size, tooth size, and bite force (Bates and Falkingham, 2012).

1530

\section{Synthesis of Ontogeny and Functional Morphology}

1532 T. rex has been central in recent quantitative studies of the functional morphology of

1533 tyrannosaurids and other large theropods (Henderson, 2002; Henderson and Snively, 2004;

1534 Therrien et al., 2005; Snively et al., 2006; Snively et al., 2019). Those results are re-examined

1535 here in the framework of the ontogram to assess the completeness of the hypotheses, provide a

1536 specific ontogenetic context for major functional changes, identify the gaps in the data, and

1537 propose hypotheses of where in the growth series major morphological changes occurred. 
1539 Orbital fenestra (Henderson, 2002). Quantifiable correlates of the height of the skull frame

1540 include the size, shape, and orientation of the orbital fenestra, the width of the pre- and

1541 postorbital bars that frame it, and the stress regime of the skull at the orbit (Henderson, 2002).

1542 One juvenile (CMNH 7541) and adult (AMNH FARB 5027) were compared by Henderson

1543 (2002; Fig. 23). Comparison of the results of Henderson (2002) with the growth curve

1544 reconstructed here (Fig. 23) shows that the details of the transition are incomplete, including the

1545 precise stage at which the transformation occurred owing to the absence of suitably complete

1546 subadult specimens from the current sample in museum collections. Given the presence of

1547 correlates of a tall skull in the exemplars of the subadult category (LACM 23845, RSM 2990.1)

1548 it is predicted here that the reduction in orbit size, increase in ellipticity, increase in vertical

1549 orientation, increase in pre- and postorbital bar length, and increase in stress regime at the orbit

1550 first occurred abruptly in subadults.

1551

1552 Skull \& jaw strength (Snively et al., 2006). A study of the bending strength of the skull and

1553 teeth of T. rex by Snively et al. (2006) included comparison between one juvenile (BMRP

1554 2002.4.1), two adults (AMNH FARB 5027, LACM 23844), and one senescent adult (FMNH

1555 PR2081). Their results were mapped onto the growth curve to obtain an ontogenetically

1556 constrained hypothesis of the comparisons of vertical bending strength, lateral bending strength,

1557 torsional strength, and tooth bending strength (Fig. 24).

1558 Snively et al. (2006) provided a summary hypothesis of the phylogenetic progression of

1559 skull strengthening attributes of tyrannosaurids. This phylogenetic progression was compared

1560 with the ontogenetic progression for T. rex found here to test the hypothesis of congruence

1561 between ontogeny and phylogeny. The sequences of changes are congruent, aside from the 
1562 occurrence of a wide adductor chamber; in Snively et al. (2006) this character is unique to T. rex,

1563 and is the last character to evolve, whereas it is present in the second growth stage as shown by

1564 its presence in CMNH 7541, a juvenile, and all specimens that are more mature than it.

1565 As in the phylogenetic transition, fused nasals are seen earliest, rostrally wide nasals are

1566 seen in large juveniles, and the increased nasal cross section, peg-in-socket nasomaxillary suture,

1567 and increased tooth strength occur simultaneously in subadults. The simultaneity is almost

1568 certainly an artifact of a low sample size, and a higher sample of subadults is required to test the

1569 hypothesis that acquisition of those features occur in the same order as is seen in the

1570 phylogenetic sequence. All of these features occur in the growth of T. rex before the $3,000 \mathrm{~kg}$

1571 threshold is reached, indicating that they are not an artifact of the descendant (i.e., T. rex) size

1572 range; indeed, these features are seen in other, smaller tyrannosaurines (e.g., Daspletosaurus, $T$.

1573 bataar, Zhuchengtyrannus).

1574 The data for maxillary tooth bending strength are from the juvenile (BMRP 2002.4.1) and

1575 adult (AMNH FARB 5027, LACM 23844) stages (Snively et al., 2006), which corresponds to

1576 the $5^{\text {th }}, 14^{\text {th }}$, and $18^{\text {th }}$ growth stages of the ontogram (Figs. 2, 24). Labiolingual tooth bending

1577 strength increases by an order of magnitude between the juvenile and adult growth stages; the

1578 juvenile bending strength is comparable to those of juvenile Daspletosaurus torosus and

1579 Albertosaurus libratus (Snively et al., 2006), which are lower than those of nontyrannosaurids.

1580 Mesiodistal bending strength increases from juvenile to adult, where that of the juvenile

1581 is most comparable to narrow-toothed Allosaurus, Monolophosaurus, and Albertosaurus libratus

1582 (Snively et al., 2006). This transition in tooth strength almost certainly occurred between the $5^{\text {th }}$

1583 and $6^{\text {th }}$ growth stages since the 7-shaped lacrimal (RSM 2990.1), regarded here as a correlate of

1584 high bites forces, is seen at the $6^{\text {th }}$ growth stage, and wide teeth (LACM 23845) are present no 
1585 later than growth stage 7. Also, the correlates of high bite forces identified by Snively et al.

1586 (2006), including a peg-in-socket nasomaxillary suture, is present in subadults (LACM 23845);

1587 the other correlates, such as a tall maxilla with a wide palatal shelf are unequivocally present by

1588 growth stage 8 (MOR 1125) and are predicted here to be present in subadults once more

1589 complete specimens are found. If true, then the teeth, along with the entire craniodental

1590 mechanism rapidly ( $<3$ years) transformed as an integrated system well in advance of somatic

1591 maturity. The increase in both tooth strength indicators among adults indicates that this trend of

1592 increasing strength continued into later growth stages; however, this observation is based on two

1593 specimens (AMNH FARB 5027, LACM 23844) and data from additional adults are required to

1594 test that hypothesis (Snively et al., 2006).

1595 Juvenile T. rex started life with snouts that are strengthened by fusion of the internasal

1596 suture and the vaulted cross section of the nasals (Snively et al., 2006). However, the

1597 dorsoventrally shallow skull and the wide and flat frontal ramus of the nasals produces a low

1598 vertical bending strength (Snively et al., 2006). The tongue-in-groove nasomaxillary joint surface

1599 and the narrow span across the nasals are correlates of a low lateral bending and low torsional

1600 strength (Snively et al., 2006).

1601 Based on their observations of adults, Snively et al. (2006) identified two correlates of

1602 high torsional strength and lateral bending strength of the skull: a peg-in-socket nasomaxillary

1603 suture and a wide span across rostral end of the nasals, which are seen in the subadult category

1604 (LACM 23845), and so the transition from a weak snout to a strong snout occurred in that earlier

1605 growth stage and was carried forward into adulthood. The change from a low to a tall skull is a

1606 central part of the transition from a weak to a strong skull (Snively et al., 2006); correlates of a

1607 tall skull, such as a wide preorbital bar (RSM 2990.1) in the subadult growth stage, shows that 
1608 the transition from shallow to tall also occurred from the juvenile to the subadult growth

1609 categories.

1610 As seen in the maxillary teeth, a trend of increased bending strength of the skull is seen

1611 among adults when the results of Snively et al. (2006) are mapped onto the ontogram (Fig. 24).

1612 However, only two specimens are sampled (AMNH FARB 5027, FMNH PR2081) and so

1613 additional adult specimens, and juveniles, are required to test this hypothesis. It is predicted that

1614 the trend of increasing bending strengths occurred throughout ontogeny, with an abrupt increase

1615 at the transition between juveniles and subadults.

1616

1617 Mandibular ramus strength (Therrien et al., 2005). Therrien et al. (2005) found that during

1618 the ontogeny of T. rex, from juvenile to adult, the rostral end of the mandible could resist high

1619 torsional loads and, between growth stages, an increase in bite force was seen. Measurements

1620 were obtained from the mid-dentary and at the second alveolus (Therrien et al., 2005). Two sets

1621 of measurements were taken, dorsoventral bending strength and relative strength (the ratio of

1622 dorsoventral and mediolateral bending strengths). The results of Therrien et al. (2005) were

1623 mapped onto the growth curve to test their hypotheses of ontogenetic change in the mandibular

1624 bending strengths of T. rex (Fig. 25).

1625 In comparison with the growth curve, dorsoventral bending strength at the second

1626 alveolus shows a progressive increase from juveniles to young adults to adults, but the trend is

1627 lost among adults (Fig. 25). This loss of pattern is not unexpected for specimens of the variable

1628 adult size. It is predicted here that the strength of subadults will be lower than adults but will

1629 found to be closer to the adult values than to those of juveniles. 
Although mid-dentary vertical bending strength is not available for juveniles, an

1631 increasing trend of strength is seen from the young adult to adult to senescent growth stages; in

1632 contrast, a clear pattern of increase is not seen among adults although the highest value is seen in

1633 the senescent adult (Fig. 25). It is predicted here that the bending strength of subadults at the

1634 mid-dentary will be less than those of the adult growth categories and that of juveniles will be

1635 significantly less than subadults.

1636 Relative strength was also estimated at the second alveolus and at the mid-length of the

1637 dentary tooth row (Therrien et al., 2005). Relative strength at the second alveolus increases from

1638 the juvenile to young adult growth categories, but a trend is not seen throughout the growth

1639 series (Fig. 25). In addition to that, values for some adults are lower (e.g., 0.72) than that for the

1640 juvenile growth stage (e.g., 0.86), indicating a high degree of variation. It is predicted here that,

1641 if there is a trend, the relative strength of subadults will be intermediate between the values for

1642 the juvenile and young adult categories.

1643 At the mid-dentary, relative strength decreases from young adults to adults, but no trend

1644 is seen among adults and senescent adults. It is predicted here, based on the trend in Fig. 25 and

1645 the values for small juvenile tyrannosaurids in Therrien et al. (2005), that the values for

1646 subadults and juveniles will be sequentially greater than those of the adult category.

1647 In short, vertical bending strength of the mandibular ramus increases ontogenetically, a

1648 trend that becomes obscured at senescence. In contrast, a trend in relative strength has a broadly

1649 decreasing trend at mid-dentary, but no clear trend at the second alveolus; i.e., more variation is

1650 seen in relative strength than in dorsoventral strength. It is predicted that data from the future

1651 discovery of subadults will be consistent with the mid-dentary pattern but will not clarify the

1652 situation at the second alveolus. 
1653

1654 Summary of craniomandibular strength. Taken together, the results of Henderson (2002),

1655 Snively et al. (2006), and Therrien et al. (2005) mapped onto the growth curve of T. rex provides

1656 evidence that the ontogenetic transformation of the skull and jaws marks an abrupt change from

1657 the juvenile morphotype to a mature morphotype. The skull of juveniles is open framed with a

1658 large orbital fenestra and low bone area, which results in low skull strength; also, the bending

1659 strength (labiolingually and mesiodistally) of the narrow teeth is low. However, a more powerful

1660 bite in juvenile T. rex relative to other theropods of the same size is indicated by the presence of

1661 a wide adductor region and fused, vaulted nasals (cf. Snively et al., 2006). The vertical and

1662 relative bending strengths of the mandibular ramus in juveniles are low, which is consistent with

1663 the low strength of the cranium.

1664 The abrupt transition to the mature morphotype (i.e., a tall skull with increase in relative

1665 bone area) is seen in subadults, where the nasals have an increased cross section, the

1666 nasomaxillary suture acquires a peg-in-socket form, and tooth strength increases (see above for

1667 correlates). Although the dorsoventral and relative bending strengths of the mandibular ramus

1668 have not been estimated for subadults, they are predicted here to be closer to the high values of

1669 adults than to the low values of juveniles.

1670 In the adult growth categories, the mature morphotype persists largely unchanged aside

1671 from continued reduction in orbit ellipticity, inclination, and area; and increase in bone area of

1672 the skull, skull strength, maxillary tooth strength, and dorsoventral and relative mandibular

1673 strength. Overall, the strength of the craniomandibular skeleton of T. rex increases throughout

1674 adulthood.

1675

Peer] reviewing PDF | (2019:12:43677:1:0:NEW 4 Apr 2020) 
1676 Ontogenetic Change and Strain Distribution

1677 Two sets of heat maps of the skull in lateral, dorsal, and ventral views were drafted to

1678 show, in relatively high-resolution, the distribution and magnitude of growth changes in each

1679 bone (Fig. 26A) and, to reflect a lower-resolution pattern, the frequency in each module (Fig.

1680 26B; Table 4). In the diagram, the percentage of changes was doubled so that the lightest shades

1681 of gray could be seen (i.e., $10 \%$ of total changes was rendered as $20 \%$ grey, and so on). In the

1682 heat map of individual bones, the greatest number of changes are seen from the orbit to the

1683 snout, specifically in the lacrimal, and maxilla, jugal, postorbital, frontal, and the dentary (Fig.

1684 26A). An intermediate number of changes are seen in the nasal, squamosal, quadratojugal,

1685 parietal, and braincase, and in the surangular and prearticular of the mandibular ramus (Fig.

1686 26A). Finally, the fewest changes of the cranium are seen in the palate and quadrate, and the

1687 angular of the lower jaw (Fig. 26A).

1688 In the heat map of the modules, the greatest amount of change is seen in the dorsal skull

1689 roof, including the nasals, lacrimal, postorbital, and frontals (Fig. 26B). An intermediate number

1690 of changes is seen in the snout, including the premaxilla, maxilla, jugal, postorbital, and palate

1691 (Fig. 26B). Finally, the fewest changes are seen in the suspensorium, parietal, and braincase, and

1692 in the mandibular ramus (Fig. 26B). In both sets of heat maps, the greatest number of changes

1693 occur in the circumorbital region whereas the fewest occur in the suspensorium, parietal,

1694 braincase, and postdentary moiety of the mandibular ramus.

1695 Rayfield (2004). These distributions were compared to two-dimensional models of strain in the

1696 cranium of T. rex (Rayfield, 2004) to test the hypothesis that the patterns of growth and strain are

1697 no different. Two models of the skull (fused, mobile) were loaded vertically (i.e., biting load)

1698 and horizontally (i.e., tearing load); compressive, tensile, and shearing stress were compared 
1699 (Rayfield, 2004). In light of recent work showing that the skull of $T$. rex was akinetic (Cost et al.,

1700 2019), the fused skull model results of Rayfield (2004) are discussed here.

1701 During a vertical bite, compressive stress loads the lower half of the skull, especially

1702 from the internal antorbital fenestra to the lower temporal bar; tensile stress is highest in the

1703 same region (Rayfield, 2004). In contrast, shear stress is highest above the orbital and internal

1704 antorbital fenestra and, to a lesser extent, along the interfenestral strut (Rayfield, 2004). The

1705 ontogenetic heat map for individual bones does not correspond to the compressive or tensile

1706 stress distributions, where the region below the orbital- and laterotemporal fenestrae (largely

1707 represented by the jugal and quadratojugal) have the lowest proportions of growth changes, with

1708 the exception of the high proportion of change seen in the maxilla and lacrimal (Fig. 26A). In

1709 contrast, the high proportion of growth changes in the lacrimal and maxilla do reflect the high

1710 shear stress seen along the corresponding dorsum of the snout and the interfenestral strut

1711 (Rayfield, 2004; Fig. 26A).

1712 In comparison with the module-based heat map, the region of lowest strain occurs where

1713 the greatest amount of change is seen (nasals, lacrimal, postorbital), and the region of highest

1714 strain corresponds to the region of an intermediate amount of growth change (maxilla, jugal; Fig.

1715 26B). However, the regions of maximum shear stress do correspond with the region of the

1716 greatest growth change, and one of the two regions of lowest shear stress (at the suspensorium),

1717 and of tensile stress, does correspond to where the lowest amount of growth changes are seen

1718 (Fig. 26B).

1719 During a horizontal tear, the compressive loadings are similar to that of a bite, except that

1720 highest loadings are seen adjacent to the loaded maxillary teeth (Rayfield, 2004). Tensile stress is

1721 similar to that of a bite, concentrated along the lower margin of the snout and orbitotemporal 
1722 regions (Rayfield, 2004). Shear stress is partly similar to the pattern seen in a bite, where stress is

1723 highest at the dorsal ramus of the lacrimal, but it is also high below the postorbital bar (Rayfield,

1724 2004). The ontogenetic heat map does not directly correspond to the stress distributions, aside

1725 from the high compressive stress on the maxilla and the shear stress seen on the lacrimal (Fig.

1726 26A). Therefore, it appears that skull growth did not track stress loads on the skull, whether

1727 imposed by biting or tearing. If skull growth was controlled by stress, then higher proportions of

1728 change would be seen in the jugal, postorbital, and suspensorium.

1729 In comparison with the module-based heat map, the pattern of maximum strain and

1730 tensile stress, along the subfenestral regions of the maxilla and jugal, do not correspond with the

1731 heat map, where the greatest changes are seen along the snout dorsum. In contrast, one of the two

1732 regions of maximum shear stress, along the snout dorsum, corresponds to the maximum number

1733 of growth changes seen there.

1734 Cost et al. (2019). Cost et al. (2019) tested hypotheses of palatal kinesis and strain in the skull of

1735 T. rex using 3-dimensional FEA modeling; the ontogenetic heat maps were compared to their

1736 results to test the null hypothesis that the ontogenetic and strain patterns are no different. Cost et

1737 al. (2019) found that strains, in each of the three loading postures (neutral, rostrocaudal,

1738 mediolateral) were concentrated along the rostrodorsal margin of the internal antorbital fenestra,

1739 palate (including the quadrate), the maxilla adjacent to the palatine, the preorbital bar, base of the

1740 postorbital bar, and the lower temporal bar.

1741 The ontogenetic heat map for individual bones is consistent with those results in that the

1742 highest number of growth changes are seen in the maxilla and lacrimal, but it is inconsistent in

1743 that the palate, and the postorbital and lower temporal bars have the lowest proportion of growth

1744 changes (Fig. 26A). Therefore, growth changes do not match the strain patterns, indicating that 
1745 growth does not follow skull loading, aside from the immediate preorbital region, and so a

1746 tightly corresponding pattern of remodeling is not seen (Fig. 26A). These results are consistent

1747 with what is seen in the stress models of Rayfield (2004).

1748 In comparison with the module-based heat map, the pattern in lateral view differs, where

1749 the regions of maximum strain (subfenestral regions of maxilla and the jugal, and the quadrate)

1750 in the model do not correspond to those of the maximum amount of growth changes (snout

1751 dorsum). In ventral view, correspondence is seen where the highest strain occurs in the palate

1752 and snout, whereas the lowest amount of strain is seen in the braincase (Fig. 26B). Prima facie,

1753 the general noncongruence between growth change distribution and strain distribution indicates

1754 that ontogenetic changes were not limited by the requirements for architectural stability and

1755 force transmission.

1756

\section{Cephalic Musculature Lever Arm and Extension (Molnar, 2013)}

1758 Molnar (2013) graphed the lever arm and extension of each adductor muscle and the 1759 mandibular depressor for juvenile (CMNH 7541) and adult (AMNH FARB 5027) T. rex, and

1760 adult $D$. torosus (CMN 8506). The anterior pterygoid muscle is excluded from this comparison,

1761 as it has been shown by Witmer (1997) that the antorbital fossa was apposed to a paranasal sinus,

1762 and not the origin for that muscle.

1763 A marked transition in the lever arm is seen from juvenile to adult in several adductors.

1764 When the mouth is closed, the lever arm increases in the posterior mandibular adductor,

1765 superficial and medial external mandibular adductor, and the mandibular depressor (Molnar,

1766 2013). In contrast, that of the deep external mandibular adductor and the pseudotemporal stays

1767 the same and that of the dorsal pterygoid decreases (Molnar, 2013). In D. torosus, the lever arms 
1768 are approximately intermediate between juvenile and adult for the posterior mandibular adductor,

1769 and the superficial and medial external mandibular adductor (Molnar, 2013).

1770 When the mouth is opened, the lever arm increases from juvenile to adult in the posterior

1771 mandibular adductor, superficial and medial external mandibular adductor, and the dorsal

1772 pterygoid, whereas that of the deep external mandibular adductor, pseudotemporal, and

1773 mandibular depressor stays the same (Molnar, 2013). In D. torosus, the lever arms are

1774 intermediate between juvenile and adult for the superficial and medial external mandibular

1775 adductor (Molnar, 2013). If the ontogenetic progression is congruent with the phylogenetic

1776 progression, it is predicted that the lever arms in subadult $T$. rex will match what is seen in $D$.

1777 torosus for the posterior mandibular adductor, and the superficial and medial external

1778 mandibular adductor when the mouth is closed, and the superficial and medial external

1779 mandibular adductor when the mouth is agape. The relative magnitude of the lever arm for the

1780 muscles is the same in all three exemplars (Molnar, 2013).

1781 A transition in percentage extension is seen from juvenile to adult T. rex, where an

1782 increase occurs in the posterior mandibular adductor, superficial, medial external mandibular

1783 adductor, and mandibular depressor (Molnar, 2013). In contrast, a decrease is seen in the

1784 pseudotemporal and the deep external mandibular adductor (Molnar, 2013). The percentage

1785 extension in D. torosus is intermediate for the posterior mandibular adductor and the superficial

1786 and medial external mandibular adductor (Molnar, 2013); assuming that the ontogenetic

1787 progression will map onto the phylogenetic transition, the expected percentage in subadult T. rex

1788 is predicted to be the same as in D. torosus. The percentage extension in D. torosus is lower than

1789 in juvenile and adult T. rex for the pseudotemporal, deep external mandibular adductor, and the

1790 mandibular depressor, indicating that the high extension of those muscles is autapomorphic in $T$. 
1791 rex (Molnar, 2013). It is predicted here that the value in subadults will be intermediate between

1792 the values for juvenile and adult $T$. rex, but it will be closer to adults than to juveniles.

1793

1794 Rotational Inertia (Henderson and Snively, 2004)

1795 Henderson and Snively (2004) quantified the rotational inertia (RI) of several

1796 tyrannosaurids, including a juvenile and adult of $A$. libratus, an adult of $D$. torosus, and an adult

1797 T. rex. Based on mass, it is predicted here that (1) the RI of juvenile T. rex (less than $500 \mathrm{~kg}$ ) will

1798 approximate that of juvenile A. libratus, (2) T. rex in the transition between subadult and adult

$1799(\sim 2,800 \mathrm{~kg})$ will be similar to that of adult A. libratus, and (3) T. rex in the young adult stage

$1800(\sim 3,800 \mathrm{~kg}$; this mass has since been revised to 3,085 kg in Snively et al., 2019) will be

1801 comparable to that of adult $D$. torosus; ergo, the ontogenetic and phylogenetic trend of

1802 tyrannosaurid RI will predict the ontogenetic RI trend of T. rex (Fig. 27).

1803

1804 Agility (Snively et al., 2019)

1805 In all tyrannosaurids, including T. rex, agility decreases from the juvenile to adult growth

1806 stage (Snively et al., 2019), a trend that continues into senescent adulthood (Fig. 28); however,

1807 the adult sample for T. rex includes only two specimens (AMNH FARB 5027, FMNH PR2081),

1808 so the trend may disappear with additional fossils. Using the mass of other tyrannosaurids as a

1809 guide, the transition from high to low agility between the tyrannosaurines is continuous, whereas

1810 the values for A. libratus are slightly lower, but are consistent with the overall trend in reduction

1811 (Snively et al., 2019; Fig. 28). Since the extreme growth changes in T. rex are concentrated in the

1812 head, there is no reason to expect an abrupt growth-related decrease of agility in the transition

1813 from juvenile to subadult. 
1814

1815 Suture Morphology

1816 Herring (1993) stated that, in vertebrates, there "seem to be no cases of beveled sutures

1817 becoming butt-ended [sensu Moss, 1957]". However, in T. rex this extreme transformation is

1818 seen at the frontonasal and surangulodentary contacts. The joint surface for the nasal on the

1819 frontals in juveniles (e.g., DDM 344.1) is a low, inclined facet, whereas in adults (e.g., MOR

1820 2822) the joint surface for the nasal is deeply incised and slot-like. The surangulodentary joint

1821 surface is a narrow, low angled and shingle-like overlapping contact in juveniles (e.g., BMRP

1822 2002.4.1), whereas in adults (e.g., MOR 980), the contact is a wide, coarse, and flattened contact

1823 at which both bones are abruptly mediolaterally expanded.

1824

1825 Suture Closure in the Braincase

1826 Although no braincase suture is unambiguously optimized as a synontomorphy, they can

1827 be visualized using the Trace function in MacClade (Maddison and Maddison, 2005). The

1828 otoccipitobasioccipital suture of the occipital condyle closes the first, no later than the start of the

1829 young adult category. This is followed by the laterosphenoidoparietal suture and the

1830 otoccipitobasioccipital suture of the subcondylar region, which close by the end of the young

1831 adult category. The laterosphenoidoprootic suture is closed by the onset of the young adult

1832 category, but there is no data for less mature specimens. Finally, neither the

1833 laterosphenoidoorbitosphenoid suture or the parietootoccipital suture shows a hierarchical

1834 pattern; both tend to remain open.

1835

1836 Encephalization Quotients 
1838 show a high Reptile Encephalization Quotient (REQ; brain mass to endocast volume ratio of

$183937 \%)$ in a juvenile (CMNH 7541) that exceeds those of adults. This growth pattern is also seen in

1840 Alligator mississippiensis (Hurlburt et al., 2013). Although adult REQs are significantly lower

1841 than what is seen in the juvenile, nonetheless an increasing progression in REQ is seen among

1842 the three specimens as first hypothesized, based on body size, by Hurlburt et al. (2013). It is

1843 naïvely predicted here that the reduction in REQ, from the juvenile value to the adult range, will

1844 coincide with the transition from juvenile to subadult growth stage as a part of the complex of

1845 changes that reshapes the skull from a shallow to a deep morphotype along with corresponding

1846 endocranial alteration.

1847

1848 Congruence between Ontogeny and Phylogeny

1849 Comparison of the frequency of phylogenetic synontomorphies (ontogenetic characters

1850 that are homologous with phylogenetic characters that have been optimized as supporting a

1851 node) and nonphylogenetic changes (ontogenetic characters that do not have a phylogenetic

1852 homologue) shows that in all cases but one, the nonphylogenetic changes exceed those of

1853 phylogenetic changes (Fig. 5). Overall they share the same peaks, except for a peak at growth

1854 stage 4 in the phylogenetic changes (Fig. 5). The corresponding 214-character region of the data

1855 matrix shows that relatively immature specimens are coded with the plesiomorphic character

1856 states, whereas relatively mature specimens are coded with apomorphic character states. It is

1857 therefore reasonable to expect that the ontogenetic character transformations would be

1858 congruent, en masse, with their phylogenetic counterparts. 
1860

1861

1862

1863

1864

1865

1866

1867

1868

1869

1870

1871

1872

1873

1874

1875

1876

1877

1878

1879

1880

1881

with the phylogenetic synapomorphies, and a limited congruence with the phylogenetic pattern recovered in Carr et al. (2017) is seen (Fig. 30, Table 19). A Shapiro-Wilk test found that the ranked growth data and clade rank data are normally distributed $(p=0.690$ and 0.767 , respectively). A Spearman-rank correlation test results in a nonsignificant $(p=0.693)$ correlation coefficient $\left(r_{s}=-0.143\right.$; Table 19, Fig. 31$)$ between growth stage rank and clade rank. The phylogenetic context of the conflicting data are illustrated in Fig. 30. It is possible that this result is an artifact of the relatively poorly sampled juvenile and subadult growth categories, which indicates that the discovery of new specimens in those categories of maturity will simultaneously increase the frequency and congruence of phylogenetically homologous character changes.

\section{Individual Variation}

By growth category, the frequency of individual variation in T. rex is, in descending order: adult (151), young adult (98), juvenile (22), senescent adult (19), subadult (6). The low amount of variation at the subadult category almost certainly results from the fact that the growth stage is represented by a three incomplete specimens (BMRP 2006.6.4, LACM 23845, RSM 2990.1). Nearly twice as much variation is seen in adults than is seen in young adults; given the completeness of specimens in these growth stages, this difference almost certainly approximates the real pattern. In nearly every skull module, the number of changes increases with ascending ontogenetic categories (Table 4), which is evidence of extensive remodeling throughout the phases of life that correspond to sexual maturity.

In comparison with the frequency of synontomorphies at each node, with two exceptions, individual variation outnumbers synontomorphies from the young adult category forward (Fig. 
1882 2). The correlation between the individual variation on each branch and the number of

1883 synontomorphies at each node was assessed under two correlation tests (Table 20). The raw data

1884 are skewed to right and were run under a Pearson correlation test that obtained a nonsignificant

$1885(p=0.559)$ correlation $(r=-0.109)$. The skewness was reduced upon converting the data to

1886 ranks (Table 20), and a Shapiro Wilk test found that the ranked data are not normally distributed

1887 for the individual variation ranks $(p=0.015)$, whereas the data for the synontomorphy ranks are

1888 normally distributed $(p=0.144)$. A Spearman rank correlation test was run, which returned a

1889 nonsignificant $(p=0.695)$ coefficient $\left(r_{S}=-0.073\right)$ (Fig. 32, Table 20). Therefore, the number of

1890 synontomorphies does not influence the amount of individual variation. A lower-resolution

1891 Spearman rank correlation test was done, at the level of growth category (Table 4), which also

1892 resulted in a nonsignificant $(p=0.397)$ correlation coefficient $\left(r_{S}=0.429\right)$, showing there is no

1893 correlation at that low level of comparison.

1894 In terms of craniomandibular modules, the frequency of individual variation, in

1895 descending order, is: dorsum of snout (100), sides of snout (57), mandibular ramus (42),

1896 braincase (29), suspensorium (23), and parietal (23) (Table 4, Data S5). As such, no region of the

1897 skull is exempt from individual variation. A second correlation test was run to compare the

1898 amount of synontomorphies with individual variation in each module (Table 21); Shapiro-Wilk

1899 normality tests found that the synontomorphy data and the individual variation data are normally

1900 distributed $(p=0.961,0.537$, respectively). The correlation test resulted in a significant $(p=$

$19010.008)$ correlation coefficient $\left(r_{s}=0.928\right)$. The similar amount of individual variation and

1902 number of synontomorphies in each skull module suggests that the number of unambiguously

1903 optimized characters are dependent upon the proportion of characters that is scored for each

1904 module in the data matrix. Indeed, a tally from the character list (Data S1) shows the frequency 
1905 of characters per module, in descending order: snout and palate (399), skull roof (334), mandible

1906 (141), suspensorium (140), braincase (85), and parietal (25). The distribution broadly matches

1907 that of the individual variation and synontomorphies, except for the reversal in order of the snout

1908 and skull roof modules, and the suspensorium and braincase modules.

1909 Among all the characters that are optimized unambiguously as individual variation, the

1910 number of reversals (from a mature to an immature character state) is close to the number of

1911 progressions (from an immature state to the mature condition), 165 to 175, respectively (Fig. 2;

1912 Data S5). Progressions are the only type of change in the first seven growth stages; after that,

1913 progressions outnumber reversals until the $11^{\text {th }}$ (exemplar: AMNH FARB 5117) whereupon

1914 reversals establish their numerically dominant and constant presence (Fig. 2; Data S5).

1915 Only eight of the 165 reversals pertain to characters of display features, which include the

1916 presence of the ridge around the external antorbital fossa of the maxilla, the texture of the dorsal

1917 ramus of the lacrimal, and the height and size of the cornual process of the postorbital (Data S5).

1918 The low frequency of variation in cephalic ornaments indicates that these presumed intraspecific

1919 display structures are not any more labile than nonornamental characteristics.

1920 In summary, individual variation is seen across the entire skeleton, although it is more

1921 frequent in the skull and jaws (274) than in the postcranium (26). Almost certainly this

1922 discrepancy is, in part, an artifact of the lower number of characters scored for postcranial bones.

1923 Individual variation is not limited to any single domain; for example, in LACM 150167, a

1924 specimen with 33 optimizations, cranial variation includes pneumatization, bone shape, muscle

1925 scar orientation, alveolus size, foramen magnum size, skull frame, neurovascular foramen

1926 position, and alveolar skirt presence, to name a few. In the same specimen, postcranial variation

1927 includes, among others, the form of the scapular shaft, prominence of a scapular muscle scar, 
1928 size of the cuppedicus fossa, form of the ischium, form of the distal joint surface on the fibula,

1929 and the orientation of the dorsal margin of the medial fossa of the fibula. As such, individual

1930 variation has an unlimited presence across the entire skeleton, which limits the precision of

1931 estimating relative maturity without a comprehensive character analysis such as the one

1932 presented here.

1933

1934 Oversplit Characters

1935 A review of the synontomophies provides the opportunity for identifying characters that

1936 are oversplit (Data S4; Table 22). An oversplit character is defined here as two or more

1937 synontomorphies that support the same node and are subsets of the same morphological

1938 structure; for example, if the glenoid fossa of the scapula and coracoid are reoriented at the same

1939 node, then it is simplest to consider them to be the same change instead of two discrete changes.

1940 In contrast, if the glenoid fossa of the scapula changes orientation at an early node, whereas the

1941 glenoid fossa of the coracoid changes at a later node, then it is reasonable to treat them as

1942 independent characters.

1943 In growth stage 5, several characters might be oversplit, including several pertaining to

1944 skull height, the antorbital fossa, dorsal margin of the postorbital, facial subcutaneous texture,

1945 and maxillary tooth count (Table 22). In growth stage 6, characters of inflation of the dorsal

1946 ramus of the lacrimal and skull frame might be oversplit (Table 22). In stage 7, oversplit

1947 characters pertain to inflation of the dorsal ramus of the lacrimal (Table 22). In stage 9, oversplit

1948 characters might pertain to alveolus size (Table 22). The test of these hypotheses is the addition

1949 of new specimens to the analysis that are sufficiently complete and of the appropriate growth

1950 stage. For instance, the great morphological differences between growth stages 4 and 5, and 5 
1951 and 6, indicate that specimens of intermediate morphology have not yet been found, and so the

1952 numerous changes seen in growth stages 5 and 6 that appear to be oversplit may have not

1953 developed simultaneously.

1954 Regardless, the effect of collapsing hypothetically overplit characters into a single

1955 transformation series was tested by running a second analysis. The transformation series were

1956 compared in three steps: (1) side-by-side comparison of possibly homologous transformation

1957 series, (2) matching transformation series were grouped together, (3) exemplar transformation

1958 series (i.e., those scored for the maximum number of specimens) were identified, and (4) the

1959 analysis was run with only the exemplar transformation series whereas the redundant

1960 transformation series were excluded from the analysis (Table 22). In cases where the

1961 transformation series are different, and so clearly test different morphologies or different aspects

1962 of the same structure, the characters were regarded as independent. Based on differences

1963 between transformation series, the characters pertaining to the antorbital fossa, dorsal margin of

1964 the postorbital, maxillary tooth count, lacrimal inflation, and alveolus size were not regarded as

1965 oversplit (Table 22).

1966 The analysis recovered 20,000 MPTs (the limit of memory capacity); a strict consensus

1967 tree shows several polytomies among juveniles (AMNH FARB 5050, CMNH 7541, DDM 344.1,

1968 LACM 28471), subadults (BMRP 2006.6.4, LACM 23845, RSM 2990.1), and adults distal to

1969 MOR 1125. Within the adult polytomy, one sister pair was recovered (MOR 555 + MOR 980).

1970 These regions of the tree have low Bremer (1-4), but relatively high bootstrap (62-97), and

1971 jackknife (61-97) values, indicating that the conflicting results are almost certainly an artifact

1972 character removal that decreased the proportion of topologically informative data.

1973 


\section{Discussion}

1975 Diagnosis of Tyrannosaurus rex. The ontogenetic position of CM 9380 among the most mature

1976 specimens in the sample (Figs. 1,2) indicates that the taxon, T. rex, is indeed based upon a

1977 mature adult and, by extension, the type is a defensible name bearer, foundation for diagnosis,

1978 and point of comparison for other specimens. The results here support one of the diagnostic

1979 features of T. rex first identified by Osborn (1905), namely gigantic size. The other characters are

1980 not diagnostic; T. rex did not have a long humerus and the absence of armor plates is

1981 symplesiomorphic for Tyrannosauridae, if not Ornthodira. Osborn (1905) observed that

1982 Dynamosaurus imperious (junior subjective synonym of T. rex) was distinguished by its number

1983 of dentary teeth "twelve to thirteen"; indeed, the results here find that T. rex has the lowest

1984 number of dentary teeth (12) among tyrannosaurids. The same is also true of the maxilla, which

1985 has as few as 11 teeth (e.g., LACM 23844).

1986 In 1906, Osborn synonymized D. imperiosus with T. rex, and he expanded the list of

1987 diagnostic characters. Of these, several are symplesiomorphies: presence of the internal

1988 antorbital fenestra and the maxillary fenestra, horizontal bar of the squamosal, presence of the

1989 subnarial foramen, small first dentary tooth, presence of interdental plates, 23 presacral

1990 vertebrae, five sacral vertebrae, sacral spinous processes fused into a single plate, atlantoaxial

1991 complex composed of six segments, reduced scapula and humerus, presence of abdominal ribs,

1992 pelvic construction, hollow limb and girdle bones, and long hind limbs.

1993 Some characters (Osborn, 1906) are mischaracterizations, such as the abbreviated skull

1994 (whereas it is dorsoventrally deep, not rostrocaudally short) and the primary metatarsals partly

1995 co-ossified (although tightly apposed, these bones are separate structures). Also, his observation

1996 of teeth in T. rex that are "very broadly oval in section, transverse exceeding anteroposterior 
1997 diameters" (Osborn, 1906: 283), deserves comment. A comparison of width to length ratios

1998 among adult T. rex and representative adults of Daspletosaurus spp. and A. sarcophagus (aside

1999 from the first two maxillary teeth and the first dentary tooth, which tend to be incisiform or

2000 conical and so incomparable with so-called lateral teeth that are ziphiform in shape) shows that

2001 dentary teeth that are wider than long have a scattered distribution among T. rex adults (Table

2002 11). Maxillary teeth with widths that exceed their lengths are not seen in T. rex, but, in general, 2003 the maxillary teeth of adult $T$. rex tend to be wider than what is seen in its close relatives, and the 2004 dentary teeth are somewhat narrower (Table 11). In contrast, the maximum width to length ratio 2005 of lateral teeth (excluding wide mesial teeth) in young adults, such as MOR 1125 , is only $68 \%$ 2006 (mean of all MOR 1125 teeth: 72\%), indicating that autapomorphically wide teeth occur late in 2007 growth. Therefore, only three of Osborn's diagnostic characters are seen here: gigantic size, the 2008 low count of twelve dentary teeth, and the extreme width of the maxillary- and mesial dentary 2009 teeth.

2010

2011 Individual variation. In T. rex individual variation increases with maturity, which contradicts

2012 the naïve expectation that variation should decrease upon the passage through an accumulation 2013 of developmental constraints throughout growth (see Results above). Evidently, constraints are 2014 tightest early in growth, as shown by the absence of character reversals from juveniles and the 2015 least mature young adult. The presence of reversals throughout adulthood is evidence that 2016 variation is less tightly constrained, allowing characters to freely reverse and progress. Also, the 2017 onset of reversals coincides with crossing the plesiomorphic 3,000 kg threshold in mass (Fig. 2).

2018 The presence of more reversals than progressions in FMNH PR2081, presumably the 2019 most mature specimen in the sample, raises the question of whether or not an individual 
2020 specimen should be considered the heuristic representative of the terminal growth stage on the 2021 ontogram. Among adult T. rex, each node is supported by a relatively low number of 2022 synontomorphies that include progressions and reversals; the characters that diagnose FMNH 2023 PR2081 are dominated by reversals, which is the pattern seen in the individual variation of other 2024 adults (AMNH FARB 5027, AMNH FARB 5029, AMNH FARB 5117, LACM 23844, MOR 2025 555, MOR 980, MOR 1131, MOR 2822, NMMNH P-3698, RSM 2523.8, SDSM 12047, UWBM 2026 99000, UWGM 191). Among synontomorphies, where both reversals and progressions are seen, 2027 in only one case do the progressions outnumber the reversals, which emphasizes the difference 2028 seen in FMNH PR2081.

2029 It is possible that the reversals might have been actual reversals over the lifetime of the 2030 individual (e.g., 0 to 1 to 0 ), or juvenile characters that were carried unchanged into adulthood.

2031 However, it is not possible to make the distinction between reversals and static character states of 2032 individuals, on the one hand, from true ontogenetic reversals on the other, without knowing the 2033 entire ontogeny of the individual organism in question. 2034

2035 Sexual maturity. Among extant archosaurs (e.g., Alligator mississippiensis), sexual maturity is 2036 reached at approximately half adult size (Wilkinson and Rhodes, 1997, in Foth et al., 2018;

2037 Wilkinson et al., 2016). If this pattern is plesiomorphic for Archosauria (Crocodylia + 2038 Dinosauria), the naïve null hypothesis for T. rex is that the onset of sexual maturity occurred 2039 when skull length reached $70 \mathrm{~cm}$. Ergo, specimens such as BMRP 2002.4.1 (skull length: $74 \mathrm{~cm}$ ) 2040 indicate that sexual maturity occurred before the onset of the extreme transition from long and 2041 low skulls to the deep and stocky frame of subadults and adults (Fig. 12). 
At the very least, it is improbable that the 15-year-old female subadult BMRP 2006.6.4

2043

2044

2045

2046

2047

2048

2049

2050

2051

2052

2053

2054

2055

2056

2057

2058

2059

2060

2061

2062

2063

represents the earliest onset of sexual maturity in T. rex (Woodward et al., 2020). If sexual

maturity occurred earlier, then T. rex is an organism with determinate growth in which growth continues after the onset of sexual maturation and stops before senescence (sensu Lincoln et al., 1982 in Wilkinson et al., 2016). As in A. mississippiensis, the growth rate in T. rex reduces once sexual maturity is reached, where it is relatively low in large juveniles and subadults (Woodward et al., 2020) and it nearly ceases in the young adults and adult categories (Horner and Padian, 2004). If these patterns of growth rate are comparable, then it is plesiomorphic and unaffected by metabolism (i.e., ectothermy in crocodylians, endothermy in T. rex). It is thought that the pattern seen in A. mississippiensis evolved as an energy expense-optimization strategy (Wilkinson et al., 2016), which might be the case for all nonavian archosaurs. Given that female $A$.

mississippiensis can produce viable eggs for their entire lives, the term "senescent" as used here for T. rex does not imply that senescent adults are nonreproductive.

Sexual dimorphism. Sexual dimorphism is marked by one or more distinct phenotypic differences between males and females, excluding differences in size (Padian and Horner, 2011). The inference of the absence of sexual dimorphism in T. rex is supported by three lines of evidence: (1) the ontogram is linear and pectinate, lacking a distinct bifurcation that unites females on one branch and males on the other; (2) the number of specimens more mature than MOR 1125 is high (22), indicating that (a) the sample size is sufficient to capture the signal of two distinct morphs and (b) the probability of sampling only one sex over the other is vanishingly low; and (3) individual variation does not separate out two identifiable groups 
2064 among subadult and adult specimens. In other words, aside from subtle differences, all adult $T$. 2065 rex simply look alike.

2066 The absence of dimorphism pertains to the osteodental characters included in the 2067 analysis; in life, dimorphism might have been expressed by differences in soft tissues (e.g., 2068 integument pigmentation) that do not preserve as fossils. The possibility that dimorphism was 2069 expressed by size and growth rate, as is seen in living crocodylians (Taylor et al., 2016), requires 2070 a sample of unambiguously female specimens, which is currently unavailable for $T$. rex. Finally, 2071 it is notable that among living crocodylians (e.g., C. yacare, Crocodylus johnstoni) dimorphism 2072 in size is seen between hatchlings, where females are smaller than males (Campos et al., 2014;

2073 Edwards et al., 2017). Therefore, should size dimorphism be found in T. rex, it might also be 2074 present, ontogenetically, from start to finish. Therefore, the absence of a basal bifurcation in the 2075 ontogram shows that early size-independent dimorphism was absent from T. rex.

Among crocodylians, size-independent sexual dimorphism is limited to the shape of the 2077 external naris in Gavialis gangeticus and Caiman latirostris and to the width of the interorbital end of the temporal roof in Crocodylus porosus (Foth et al., 2013). In contrast, variation in the 2079 shape of the bony naris and the interorbital region in T. rex is ontogenetically controlled and 2080 dimorphism is not seen. In summary, T. rex shows the pattern of variation (high ontogenetic 2081 variation, dimorphism absent, high individual variation) that is expected where only species 2082 recognition is at work (Padian and Horner, 2011).

2083

2084 Size, maturity, and age. The suggestion has recently been made that size might correlate with 2085 maturity in T. rex (Persons et al., 2019), which was based on histological evidence. The 2086 specimen in question (RSM 2523.8), the youngest adult identified here, was hypothesized to 
2087 exceed FMNH PR2081 in maturity based on the presence of a high degree of remodeling in the 2088 fibula that has produced a dense system of secondary osteons (Persons et al., 2019). It is 2089 expected that if remodeling is congruent with maturity, then on the ontogram RSM 2523.8 would 2090 share a most recent growth stage with FMNH PR2081 to the exclusion of all other specimens. 2091 Among living archosaurs (e.g., Melanosuchus niger, Caiman yacare) variation in size is 2092 high, which is evidenced by high standard deviations (e.g., Platt et al., 2011; Taylor et al., 2016), 2093 wide 95\% confidence intervals (Campos et al., 2014), individual variation in growth rates 2094 (Campos et al., 2014), and by the relative inaccuracy of age estimation from size (Campos et al., 2095 2014). Individual variation tends to be high among large, mature animals (e.g., C. acutus; Platt 2096 et. al., 2011). Also, agreement between growth rate data and growth curve models are dependent 2097 upon sample size (Taylor et al., 2016). This suggests that currently the sample size for T. rex 2098 (seven specimens in Erickson et al., 2004) is insufficient for accurately capturing the congruence 2099 between mass and age.

2100 Also, size in crocodylians is affected by multiple extrinsic variables, including 2101 seasonality, seasonality of growth, incubation conditions, mean temperature (although this effect 2102 is not always found; Campos et al., 2014), rainfall, prey type, prey abundance, population 2103 density, social milieu, and disease (Campos et al, 2014, Taylor et al., 2016). Although not all of 2104 these variables would have affected $T$. rex, an endothermic dinosaur, to the same extent that is 2105 seen in ectothermic crocodylians, it is not surprising that the sizes seen among the most mature 2106 specimens are not congruent with maturity. Alternatively, if extrinsic factors had a negligible 2107 effect upon body size, then sexual dimorphism might explain the noncongruence between 2108 maturity and size (but as shown, it does not). Among living crocodylians, linear measurements of 
2109 adult animals are more variable than what is seen in juveniles (Platt et al., 2011), and T. rex is

2110 consistent with that pattern.

2111 In A. mississippiensis, growth rate effectively ceases at less than half the maximum age

2112 (Wilkinson et al., 2016); a similar pattern is seen in T. rex, where growth rate is greatly reduced

2113 at slightly greater than half the maximum age (18 to 28 years; Horner and Padian, 2004). The

2114 relatively wide range of size and mass for T. rex (Erickson et al., 2004; Persons et al., 2019;

2115 Snively et al., 2019;) has two possible explanations: (1) it had determinate growth type II (sensu

2116 Sebens, 1987 in Wilkinson et al., 2016) where although final size is genetically controlled it is

2117 affected by extrinsic factors, resulting in a wide range of adult sizes; alternatively (2) it had

2118 determinate growth type I where it has sexual size dimorphism, resulting in the scatter of adult

2119 sizes, but the environment has little effect upon asymptotic size. Therefore, the evidence shows

2120 that T. rex had determinate type II growth.

2121 The variation in size among the categories of adult (i.e., the noncongruence between size

2122 and maturity) is evidence of determinate growth, where the greatest amount of variation in size is

2123 seen that reflects the idiosyncratic differences in accumulated growth rate between individuals.

2124 In other words, the predictive value of size in T. rex ceases once the individual asymptote is

2125 reached (Monteiro et al., 1997; Tucker et al., 2006).

2126

2127 Plesiomorphic Archosauriform Growth Patterns

2128 A literature review of craniomandibular ontogeny in extinct (e.g., Bhullar et al., 2012;

2129 Ezcurra and Butler, 2015; Foth et al., 2016; Horner and Goodwin, 2006, 2009) and extant (e.g.,

2130 Dodson, 1975 ; Piras et al., 2010 in Foth et al, 2013; Platt et al., 2011; Wu et al., 2006 in Foth et 
2131 al., 2013) archosaurs was done to identify the ancestral growth trends inherited by $T$. rex from its

2132 archosauriform ancestor.

2133 Evidence for growth trends established in the common ancestors Archosauriformes (i.e.,

2134 the common ancestor of Proterosuchus + Archosauria, and all of its descendants, living and

2135 extinct), Archosauria (i.e., the common ancestor of Crocodylia + Dinosauria, and all of its

2136 descendants, living and extinct), Dinosauria (i.e., the common ancestor of Triceratops and

2137 Passer, and all of its descendants, living and extinct), Saurischia (i.e., the common ancestor of

2138 Massospondylus + T. rex, and all of its descendants, living and extinct), and Neotheropoda (i.e.,

2139 the common ancestor of Coelophysis + T. rex, and all of its descendants, living and extinct), were

2140 drawn from comparisons between T. rex, a sample of saurischian dinosaurs (Bhullar et al., 2012;

2141 Foth et al., 2016), extant crocodylians, and the extinct Proterosuchus (Ezcurra and Butler, 2015);

2142 the comparisons are summarized in Table 23. The character states were optimized onto a

2143 topology that was reconstructed in MacClade (Maddison and Maddison, 2005) to identify

2144 ancestral states in the phylogenetic hierarchy. The informal terms "archosauriforms" and

2145 "archosaurs" are used in place of their formal names in this discussion; the name Tyrannosaurus

2146 bataar is used in place of "Tarbosaurus." A summary of the phylogenetic framework, the taxa

2147 included in this comparison, and the results are shown in Fig. 33.

2148 Dorsoventral deepening of the skull frame. Ontogenetic deepening of the skull frame comes

2149 from several lines of evidence, including the dorsoventral height of skull regions, specific bones,

2150 and fenestrae. In the archosauriforms Tyrannosaurus rex, crocodylians and Proterosuchus, the

2151 horizontal ramus of the maxilla increases in dorsoventral height (Ezcurra and Butler, 2015) (Fig.

$215233)$. 
Another correlate of increased skull height in archosauriforms is the decreased height of

2154 the postorbital that is seen in T. rex, where the contribution of the bone to skull height is reduced

2155 by the dorsoventral expansion of the jugal, and Proterosuchus, where its height relative to total

2156 skull length decreases (Ezcurra and Butler, 2015). A reduction in the length of the orbital

2157 fenestra is another correlate of skull deepening, which is seen in Coelophysis (Bhullar et al., 2158 2012) and T. rex.

2159 In archosaurs, the snout and jugal of crocodylians increase in depth, as also occurs in $T$.

2160 rex, in Melanosuchus niger (Foth et al., 2013), C. acutus, Mecistops cataphractus, and

2161 Tomistoma schlegelii (Foth et al., 2013); likewise, a shallow snout is a feature shared between

2162 juvenile caimans (Foth et al., 2018) and Tyrannosaurus rex. This change is one of several

2163 correlates of the ontogenetic increase in height of the entire skull frame. Analysis of

2164 morphometric data quantifies the deepening of the skull, which is seen in Massospondylus, the

2165 megalosaurid Dubreuillosaurus, Allosaurus, and T. bataar (Foth et al., 2016). Optimization of

2166 the trends of increase in snout height and skull height onto the phylogenetic topology shows that

2167 both are ancestral for Archosauriformes, a trend that is not seen in Coelophysis (Fig. 33).

2168 Expansion of the adductor region. In the archosauriforms T. rex, Proterosuchus, and all

2169 crocodylian taxa, the orbital fenestra is ontogenetically reduced in size, a correlate of adductor

2170 expansion (Ezcurra and Butler, 2015; Foth et al., 2013; Foth et al., 2018). In T. rex and

2171 Proterosuchus the orbital fenestra decreases in length and increases in height (Ezcurra and

2172 Butler, 2015). Ergo, this reduction in size is almost certainly the result of two processes, namely

2173 the rostrocaudal expansion of the adductor chamber and dorsoventral expansion of the skull

2174 frame. The adductor region, including the laterotemporal fenestra, increases in height in T. rex

2175 and Proterosuchus (Ezcurra and Butler, 2015). Geometric morphometric comparison shows that 
2176 the adductor region is not expanded in Massospondylus or Coelophysis, whereas it is expanded

2177 in Dubreuillosaurus, Allosaurus, and T. bataar (Foth et al., 2016). Optimization of character

2178 states onto the phylogenetic topology recovers adductor chamber expansion as plesiomorphic for

2179 Archosauriformes (Fig. 33).

2180 Mediolateral widening of the skull frame. In archosauriforms, an overall increase in snout

2181 width is seen in T. rex and in the crocodylians Alligator sinensis (Wu et al., 2006 in Foth et al.,

2182 2013), Crocodylus acutus, Mecistops cataphractus, and Tomistoma schlegelii (Piras et al., 2010

2183 in Foth et al, 2013; Platt et al., 2011), and also in A. mississippiensis (Dodson, 1975 in Foth et

2184 al., 2013) and C. novaeguineae (Hall and Portier, 1994 in Foth et al., 2013). Widening of the

2185 snout in crocodylians is thought to coincide with changes in diet, for example from invertebrates

2186 to vertebrates (Platt et al., 2011).

2187 An ontogenetic increase in frontal (=interorbital) width is seen in Tyrannosaurus rex and

2188 all crocodylians except $A$. sinensis (Wu et al., 2006; Foth et al., 2013; Edwards et al., 2017). The

2189 ontogenetic widening of the parietals that is seen in Proterosuchus might be homologous to the

2190 increase in width seen in T. rex and crocodylians (Ezcurra and Butler, 2015).

2191 Ontogenetic widening of the skull in archosaurs is indicated by correlates seen in the

2192 snout and dorsal skull roof. In T. rex, caimans (e.g., C. latirostris, C. sclerops, C. yacare), and

2193 crocodiles (e.g., Crocodylus acutus) the rostral end of the snout widens (Platt at al., 2011). The

2194 premaxilla shortens in Melanosuchus niger (Foth et al., 2013) and Coelophysis (Bhullar et al.,

2195 2012), which corresponds to the widening and medialward reorientation of the premaxilla (i.e.,

2196 increase in snout width) in T. rex. Optimization of skull widening on the topology recovers it as

2197 ancestral for Archosauriformes (Fig. 33). 
2198 Antorbital fossa length. Geometric morphometric comparisons recover a shortening of the

2199 antorbital fossa during growth, a trend that is ancestral at least for Saurischia, aside from an

2200 apomorphic lengthening in Coeolophysis (Foth et al., 2016; Fig. 33). This landmark-based

2201 comparison has not been done for Proterosuchus or T. rex, but it is predicted here that it will be

2202 recovered in both taxa and, hence, the trend is plesiomorphic for Archosauriformes.

2203 Jaw joint position. Geometric morphometric comparisons have recovered the dorsoventral and 2204 rostrocaudal growth trends of the cranial jaw joint for Proterosuchus (Ezcurra and Butler, 2015) 2205 and Saurischia (Foth et al., 2016). Optimization of jaw joint position recovers a caudalward shift 2206 as the ancestral state for Archosauriformes, with two independently acquired apomorphic rostral 2207 shifts in Massospondylus and Allosaurus (Fig. 33). It is predicted here that T. rex will be found to 2208 have the same caudalward shift with T. bataar. Likewise, landmark-based comparisons (Ezcurra 2209 and Butler, 2015; Foth et al., 2016) show that a ventralward shift of the jaw joint is

2210 plesiomorphic for Archosauriformes, with an apomorphic dorsalward shift in Coelophysis and no 2211 dorsoventral shift in Dubreuillosaurus (Fig. 33). It is predicted here that T. rex will be found to 2212 share a ventralward shift of the jaw joint with its sister species, T. bataar.

2213 Dorsoventral deepening of the mandibular ramus. The ontogenetic deepening of the

2214 mandibular ramus in archosaurs is evidenced by the entire ramus and by the external mandibular

2215 fenestra. In T. rex and crocodylians the external mandibular fenestra and the mandibular ramus 2216 increase in dorsoventral height (Monteiro and Soares, 1997). Optimization of the trend of 2217 increasing mandible height onto the phylogenetic topology recovers it as a synapomorphy of 2218 Archosauria (Fig. 33). The lower jaw was not included in the study of Proterosuchus ontogeny 2219 and so it is unknown if the mandible deepened as well (Ezcurra and Butler, 2015). 
2220 Increase in maxillary tooth count. An ontogenetic increase in tooth count of archosauriforms is

2221 seen in T. rex and Proterosuchus (Ezcurra and Butler, 2015); in contrast, tooth number is

2222 ontogenetically constant in extant crocodylians (Brown et al., 2015). Optimization of the trend in

2223 tooth count onto the phylogenetic topology recovers an increase in number as plesiomorphic for

2224 Archosauriformes (Fig. 33). The presence of ontogenetic tooth increase in other dinosaurs and the

2225 extant sister clade of Archosauria, Squamata, indicates that the pattern seen in crocodylians is the

2226 apomorphic condition (Brown et al., 2015).

2227 Bite force. A growth-related increase in bite forces is seen in crocodylians and in T. rex, a trend

2228 that is continued into adulthood (Bates and Falkingham, 2012; Erickson et al., 2003, 2013;

2229 Gignac and Erickson, 2014, 2017; Gignac and O’Brien, 2016). Optimization of this trend onto

2230 the phylogenetic topology recovers increasing bite force as a synapomorphy of Archosauria; it is

2231 expected that this trend is pleiomorphic for Archosauriformes, once data are obtained for

2232 Proterosuchus or other basal taxa.

2233 Cephalic ornamentation. Growth-related reduction in cephalic ornamentation is seen in

2234 Triceratops (Horner and Goodwin, 2006), Pachycephalosaurus (Horner and Goodwin, 2009),

2235 and in T. rex. Optimization of the growth trend onto the phylogenetic topology recovers this

2236 pattern as synapomorphic for Dinosauria (Fig. 33); it is predicted that this trend will be an

2237 archosauriform synapomorphy as appropriate data are documented.

2238 Appendicular skeleton. T. rex shares two postcranial growth changes with Coelophysis (Griffin,

2239 2018), which indicates synapomorphic growth changes that possibly unite Neotheropoda. These

2240 include the thickened deltopectoral crest of the humerus and enhancement of the trochanteric

2241 shelf of the femur. When optimized onto the phylogenetic topology, both trends are recovered as 
2242 synapomorphies of Neotheropoda; it is predicted that these trends will be found to be

2243 synapomorphies of Archosauriformes.

2244 Summary. Taken together, the ancestral growth pattern of the archosauriform skull that was

2245 inherited by T. rex, was marked by (1) increase in height of the entire skull, (2) rostrocaudal and

2246 dorsoventral expansion of the adductor chamber, (3) increase in skull width, (4) caudalward shift

2247 of the jaw joint, (5) ventralward shift of the jaw joint, and an (6) increase in maxillary tooth

2248 number (Fig. 33). The ancestral growth pattern of the archosaurian skull that was inherited by $T$.

2249 rex included (1) increase in mandible height and (2) an increase in bite force. The ancestral

2250 growth pattern of the dinosaurian skull that was inherited directly by $T$. rex was the reduction in

2251 cephalic ornamentation. The ancestral growth pattern of the saurischian skull that was inherited

2252 by T. rex included the decrease in the length of the antorbital fossa. The ancestral growth pattern

2253 of Neotheropoda that was inherited directly by T. rex included increase in size of (1) the

2254 deltopectoral crest of the humerus and (2) the trochanteric crest of the femur. It is expected that

2255 the more exclusive trends will eventually be seen in Proterosuchus and other basal

2256 archosauriformians once adequate data are obtained.

2257

2258 Early Onset of Adult Skull Shape

2259 The unique skull shape of $T$. rex, in having a mediolaterally narrow snout but wide

2260 orbitotemporal region (Figs. 12, 26), is seen in the youngest of the most complete specimens in

2261 the sample (e.g., CMNH 7541) that is approximately $40 \%$ of adult skull length. This

2262 phenomenon of the presence of the adult shape in juveniles (i.e., developmental capture; sensu

2263 Padian, 1995) is not unusual among archosaurs; for example, it is also seen in caimans (Monteiro

2264 and Soares, 1997). This phenomenon is notable in that the adult skull shape in $T$. rex is derived 
2265 in contrast to its closest relatives, but it ontogenetically precedes the occurrence of the suite of

2266 other evolutionary novelties that sets T. rex apart from T. bataar and other advanced

2267 tyrannosaurines.

2268 This observation is evidence that skull shape in T. rex is not recapitulatorily locked to a

2269 later growth stage; rather, continuity is seen in skull function and behavior between juvenile and

2270 adult $T$. rex, and niche partitioning between those small and large growth stages was primarily

2271 governed by increased bite force instead of fundamental differences in sensorimotor integration

2272 and functional morphology (aside from the consequences of the corresponding increase in skull

2273 and jaw height) and mode of forage. From its earliest growth stage, T. rex had already

2274 fundamentally departed from its ancestral skull morphology and, presumably, function.

2275

2276 Juvenile to 'Adult' Skull Morphotype Transition

2277 The results found here show that the extreme ontogenetic transition in skull shape of $T$.

2278 rex is often mischaracterized as between juvenile to adult, whereas the switch is actually much

2279 earlier, between juvenile and subadult. In living crocodiles, transitions in skull shape mark

2280 changes in $\operatorname{diet}$ (e.g., C. acutus; Platt et al., 2011), and the same is doubtlessly true in T. rex.

2281 Aside from skull shape in living crocodiles (e.g., C. niloticus), size is an important factor that

2282 drives juveniles from their natal river range to a lacustrine habitat (Hutton, 1989); likewise, the

2283 extreme transition in skull shape in T. rex might also mark a dispersal stage with movement

2284 from, say, a closed habitat that provided protection from adults to an open setting where safety

2285 from conspecifics was no longer a priority. Presumably, the deep-skulled morphotype resulted in

2286 an expansion of range among subadults and adults, where females would be limited to nesting 
2287 areas and males would have been more widely distributed across the landscape (cf. Hutton, 2288 1989).

2289

2290 Ontogeny and Module Integration

2291 Uniform changes across the skull implies functional integration of the skull in contrast to 2292 modularity (Monteiro et al., 1997). In T. rex, the skull modules follow the same general pattern 2293 of change, starting with few changes $(<10)$, followed by a peak $(>10)$ from the $5^{\text {th }}$ to $6^{\text {th }}$ growth 2294 stages, followed thereafter by a low rate of change $(<10)$ (Table 4, Fig. 11). The skull roof has a 2295 peak of change at growth stage 21, showing its independence from the other modules. Beyond 2296 that instance, the modules do not tend to change independently from each other from young 2297 adulthood onwards, indicating that the modules are tightly integrated with each other, which is 2298 consistent with the akinetic hypothesis (Cost et al., 2019) in contrast to the flexible skull 2299 hypothesis (cf. Werneburg et al., 2019).

2300

2301 Predictions

2302 (1) EFS will be found in AMNH FARB 5027, AMNH FARB 5029, BM R7994, CM 9380, 2303 LACM 23844, MOR 008, MOR 980, MOR 1131, MOR 2822, NMMNH P-3698, UCMP 2304 118742, UMNH 11000, and UWGM 181, should appropriate bones be available for analysis. 2305 However, an EFS is absent from MOR 555 (Horner and Padian, 2004), which suggests that the 2306 congruence between the presence of an EFS, maturity, and chronological age is not tightly 2307 constrained. Specific predictions of LAG-based chronological ages for specimens in this study 2308 are given in Table 24.

2309 (2) EFS will be absent from AMNH FARB 5117, LACM 150167, and UWBM 99000. 
2310 (3) If senescence in T. rex does not imply reproductive nonviability, then medullary bone will be

2311 found in pregnant senescent females.

2312 (4) If the distinct ridges of the subcutaneous surface of the maxilla result from ossification of the

2313 dermis, then histological sections will show an overlay of metaplastic bone apposed to the

2314 pericortical surface (sensu Horner and Goodwin, 2009). I.e., if flat facial scales precede the onset

2315 of armor-like skin and ossification of the dermis, then histological analysis will show a transition

2316 from the absence of metaplastic bone to its presence.

2317 (5) If secondary metamorphosis is present, the relationship between bite force and independent

2318 measures of body size will be nonlinear.

2319 (6) In terms of quantitative functional morphology, complete subadult skulls will be found to be

2320 more similar to adults than to juveniles, indicating an abrupt and wholesale morphological

2321 transition from low to tall skulls, whereas the postcranium will show a continuum from juvenile

2322 to adult.

2323

2324 Conclusions

2325 (1) A single growth series for T. rex was obtained that can be divided into 21 growth stages;

2326 FMNH PR2081 was recovered as the most mature specimen, whereas RSM 2523.8 is one of the

2327 least mature adults.

2328 (2) Specimens coded with as few as $1.8 \%$ of the characters are in the backbone ontogram.

2329 (3) Specimen completeness does not influence the number of unambiguously-optimized

2330 synontomorphies that support each node. 
2331 (4) Five growth categories (juvenile, subadult, young adult, adult, senescent adult) were

2332 diagnosed based on histology, synontomorphies, and, in part, size and mass; sharp boundaries

2333 between categories are seen at the subadult and adult categories.

2334 (5) The sample is numerically biased toward specimens in the subadult, young adult, adult, and

2335 senescent adult categories; i.e., there is a large gap in the growth series at the juvenile to subadult

2336 transition. Also, juveniles are underrepresented in the sample.

2337 (6) The problematic specimen TMM 41436-1 is a subadult, which accounts for its differences

2338 from adults.

2339 (7) Phylogenetic and nonphylogenetic changes follow a similar frequency distribution;

2340 phylogenetic changes are more frequent early in ontogeny (growth stages 5 to 9) than later in 2341 growth.

2342 (8) Mandibular changes are completed before cranial changes.

2343 (9) The dorsotemporal fossa of the frontal was an origin for adductor musculature.

2344 (10) The greatest number of growth changes are seen in the large juvenile and subadult growth

2345 categories, at growth stages 5 and 6.

2346 (11) The number of growth changes generally decreases during adulthood.

2347 (12) The skull roof module undergoes the most growth change.

2348 (13) Pneumatic changes are dominated by the antorbital (=paranasal) air sac system.

2349 (14) The skull frame is dominant among apneumatic changes in the skull and mandible.

2350 (15) Decreases in maxillary and dentary tooth count are broadly congruent with maturity; tooth

2351 count in both bones initially increases before it decreases.

2352 (16) Most braincase sutures close during the young adult category, whereas two stay open. 
2353 (17) Changes to the pectoral girdle and pes are dominant early in ontogeny, whereas axial and 2354 pelvic girdle changes occur late; the fibula changes throughout. The most postcranial changes 2355 happen to the fibula.

2356 (18) Body size and mass are not congruent with maturity during adulthood.

2357 (19) Maturity is congruent with chronological age, bite force, REQ, the presence and depth of the 2358 dentary groove, and decrease in agility.

2359 (20) Maturity is incongruent with geographic location, stratigraphic position, phylogeny, and 2360 ischial divergence.

2361 (21) The transition from a long and low skull to a stout and deep skull (i.e., the juvenile-subadult 2362 transition) occurred rapidly within a two-year time span. Ergo, the osteological correlates of 2363 skull strength occurred before adult size (i.e., somatic maturity) was reached.

2364 (22) T. rex exceeded the plesiomorphic size and mass of Tyrannosauridae between its $15^{\text {th }}$ and $236518^{\text {th }}$ years.

2366 (23) Sexual maturity in T. rex occurred before its $15^{\text {th }}$ year and as early as its $13^{\text {th }}$ year. Ergo, as 2367 in crocodylians and other reptiles, including non-avian dinosaurs (Erickson et al., 2007; Lee and 2368 Werning, 2008), T. rex reached sexual maturity before reaching asymptotic adult size.

2369 (24) Skeletodental sexual dimorphism is absent from T. rex.

2370 (25) There is no evidence for the taxon that is informally dubbed Tyrannosaurus " $x$ ".

2371 (26) The entire skeleton is affected by individual variation; reversals of individual variation did 2372 not occur until young adulthood (growth stage 8).

2373 (27) Individual variation affects the snout dorsum the most; display features are not

2374 disproportionately affected by individual variation. 
2375 (28) The variability in adult size implies that $T$. rex had determinate growth type II (sensu 2376 Sebens, 1987).

2377 (29) Growth patterns of the skull do not reflect the distribution of stress loads upon it.

2378 (30) The adductor chamber rostrocaudally expands at the young adult growth stage.

2379 (31) Removal of redundant putative oversplit characters results in loss of topological resolution, 2380 which is almost certainly an indication of the patchiness of the character matrix.

2381 (32) The type specimen is an adult; three of the characters of Osborn's diagnosis of the taxon are 2382 defensible.

2383

2384 Acknowledgements

2385 For access to specimens I thank: M. Norell, C. Mehling (AMNH); S. Chapman (BM); M.

2386 Henderson, J. Mathews, S. Williams (BMRP); M. Dawson, M. Lamanna (CM); M. Ryan, M.

2387 Williams (CMNH); P Makovicky, W. Simpson, A. Stroup (FMNH); L. Chiappe, S. McLeod, M.

2388 Walsh (LACM); A. Atwater, B. Helms, J. Horner, J. Scannella (MOR); T. Williamson

2389 (NMMNH); D. Evans, K. Seymour (ROM); T. Tokaryk (RSM); M. Pinsdorf (SDSM); D.

2390 Brinkman, D. Henderson; B. Strilisky (RTMP); M. Holland, G. Wilson (UWBM); C. Eaton

2391 (UWGM). For photographs of TMM 41436-1 I thank T. Adams; I thank T. Williamson for

2392 images of NMMNH P-3698. For room and board during extended collections visits, I thank J.

2393 Scannella, K. Scannella, K. Tremaine, S. Williams (MOR); T. Williamson (NMMNH); K.

2394 Seymour (ROM); D. Brinkman, D. Henderson (RTMP); M. Holland (UWBM). I thank the

2395 landowners who donated specimens from their private land to public trusts, namely the Buyse

2396 (MOR 008) and the Skillman (MOR 002) families. For discussion I thank J. Miller (Carthage

2397 College) and T. Miller. For assistance with locality data and maps I thank E. Berry (SDSM), C. 
2398 Levitt-Bussian (UMNH), A. McGee (CMNH), C. Eaton (UWGM), A. Henrici (CM), P. Holroyd 2399 (UCMP), M. Lamanna (CM), G. Liggett (BLM), D. Malinzak (Black Hills State University, 2400 Spearfish, SD), R. McKellar (RSM), C. Mehling (AMNH), W. Simpson (FMNH), S. Williams 2401 (MOR), T. Williamson (NMMNH), G. Wilson (UWBM), and the US Army Corps of Engineers. 2402 I thank D. Evans (ROM) for discussion of HCF stratigraphy and references; and J. Clarke (UT2403 Austin) for discussion of sexual dimorphism in Dinosauria. I thank M. Seitz for reviewing the 2404 character list and A. Zietlow for reviewing the manuscript, figures, and tables prior to 2405 submission. I thank C. Sabbar for technical help through Library Services (Carthage College) 2406 and P. Kivolowitz (Carthage College) for additional technical support. The specimens DDM 2407344.1 and DDM 1536.8 were collected under BLM Paleontological Resources Permits M 2408103845 and MTM 110440 (issued to TDC), respectively, and G. Liggett, D. Melton, and G. 2409 Smith (all of the BLM) are thanked for facilitating the approval process. Finally I thank the 2410 reviewers (anonymous, D. Barta, J. Frederickson), the primary editor (A. Farke) for their helpful 2411 comments that greatly improved the first draft, and journal staff editors (P. Binfield, E. Jung, S. 2412 Kusy, S. Johnson) for their helpful feedback and facilitation during the review process. 


\section{References}

2415

2416

2417

2418

2419

2420

2421

2422

2423

2424

2425

2426

2427

2428

2429

2430

2431

2432

2433

2434

2435

2436

2437

2438

2439

2440

2441

2442

2443

2444

2445

2446

2447

2448

2449

2450

2451

2452

2453

2454

2455

2456

2457

2458

Agur, A. M. R. and M. J. Lee. 1991. Grant's Atlas of Anatomy. Ninth Edition. Baltimore: Williams and Wilkins.

Amerise, I. L., and A. Tarsitano. 2015. Correction methods for ties in rank correlations. Journal of Applied Statistics: 42: 2584-2596.

Bakker, R.T., M. Williams, and P.J. Currie. 1988. Nanotyrannus, a new genus of pygmy tyrannosaur, from the latest Cretaceous of Montana. Hunteria 1: 1-30.

Bates, K. T., P. L. Manning, D. Hodgetts, and W. I. Sellers. 2009. Estimating mass properties of dinosaurs using later imaging and 3D computer modeling. PLoS ONE 4: e4532.

Bates, K. T. and P. L. Falkingham. 2012. Estimating maximum bite performance in Tyrannosaurus rex using multi-body dynamics. Biology Letters: doi:10.1098/rsbl.2012.0056.

Bates, K. T. and P. L. Falkingham. 2018. Correction to 'Estimating maximum bite performance in Tyrannosaurus rex using multi-body dynamics'. Biology Letters 14: 20180160. Doi: 10.1098/rsbl.2018/0160.

Bhullar, B.-A. S., J. M. Marugán-Lobón, F. Racimo, G. S. Bever, T. B. Rowe, M. A. Norell, and A. Abzhanov. 2012. Birds have paedomorphic dinosaur skulls. Nature. DOI: 10.1038 /nature11146.

Brazeau, M. D. 2011. Problematic character coding methods in morphology and their effects. Biological Journal of the Linnean Society 104: 489-498.

Brinkman, D. 1988. Size-indpendent criteria for estimating relative age in Ophiacodon and Dimetrodon (Reptilia, Pelycosauria) from the Admiral and lower Belle Plains formations of west-central Texas. Journal of Vertebrate Paleontology 2: 172-180.

Brochu, C. A. 1996. Closure of neurocentral sutures during crocodilian ontogeny: implications for maturity assessment in fossil archosaurs. Journal of Vertebrate Paleontology 16: 4962 .

Brown, C. M., C. S. VanBuren, D. W. Larson, K. S. Brink, N. E. Campione, M. J. Vavrek, and D. C. Evans. 2015. Tooth counts through growth in diapsid reptiles: implications for interpreting individual and size-related variation in the fossil record. Journal of Anatomy 226: 322-333.

Brusatte, S.L., M.A. Norell, T.D. Carr, G.M. Erickson, J.R. Hutchinson, A.M. Balanoff, G.S. Bever, J.N. Choiniere, P.J. Makovicky, and X. Xu. 2010. Tyrannosaur paleobiology: new research on ancient exemplar organisms. Science 329: 1481-1485.

Peer) reviewing PDF | (2019:12:43677:1:0:NEW 4 Apr 2020) 
2459

2460

2461

2462

2463

2464

2465

2466

2467

2468

2469

2470

2471

2472

2473

2474

2475

2476

2477

2478

2479

2480

2481

2482

2483

2484

2485

2486

2487

2488

2489

2490

2491

2492

2493

2494

2495

2496

2497

2498

2499

2500

2501

2502

2503

Brusatte, S.L. and T.D. Carr. 2016. The phylogeny and evolutionary history of tyrannosauroid dinosaurs. Scientific Reports 6: 20252, doi: 10.1038/srep20252.

Campione, N. E., D. C. Evans, C. M. Brown, and M. T. Carrano. 2014. Body mass estimation in on-avian bipeds using a theoretical conversion to quadruped stylopodial proportions. Methods in Ecology and Evolution 5: 913-923.

Campos, Z., G. Mourão, M. Coutinho, and W. E. Magnusson. 2014. Growth of Caiman crocodilus yacare in the Brazilian Pantanal. PLoS ONE 9: e89363.

Carpenter, K. 1990. Variation in Tyrannosaurus rex. Pp. 141-145, K. Carpenter and P. J. Currie (eds.), Dinosaur Systematics: Approaches and Perspective. University of Cambridge Press.

Carr, T.D. 1999. Craniofacial ontogeny in Tyrannosauridae (Dinosauria: Coelurosauria). Journal of Vertebrate Paleontology 19: 497-520.

Carr, T. D. 2010. A taxonomic assessment of the type series of Albertosaurus sarcophagus and the identity of Tyrannosauridae (Dinosauria, Coelurosauria) in the Albertosaurus bonebed from the Horseshoe Canyon Formation (Campanian-Maastrichtian, Late Cretaceous). Canadian Journal of Earth Sciences 47: 1213-1226.

Carr, T. D., and T. E. Williamson. 2004. Diversity of late Maastrichtian Tyrannosauridae (Dinosauria: Theropoda) from western North America. Zoological Journal of the Linnean Society 142: 479-523.

Carr, T. D., D. J. Varricchio, J. C. Sedlmayr, E. M. Roberts, and J. R. Moore. 2017. A new tyrannosaur with evidence for anagenesis and crocodile-like facial sensory system. Scientific Reports 7: 44942.

Carrano, M. T., R. B. J. Benson, and S. D. Sampson. 2012. The phylogeny of Tetanurae (Dinosauria: Theropoda). Journal of Systematic Palaeontology 10: 211-300.

Colbert, M. W. and T. Rowe. 2008. Ontogenetic sequence analysis: using parsimony to characterize developmental sequences and sequence polymorphism. Journal of Experimental Zoology 310B: 398-416. Doi.org/10.1002/jez.b.21212.

Cost, I. N., K. M. Middleton, K. C. Sellers, M. S. Echols, L. M. Witmer, J. L. Davis, and C. M. Holliday. 2019. Palatal biomechanics and its significance for cranial kinesis in Tyrannosaurus rex. The Anatomical Record: 1-19. DOI: 10.1002/ar.24219.

Currie, P. J. 2003. Allometric growth in tyrannosaurids (Dinosauria: Theropoda) from the upper Cretaceous of North America and Asia. Canadian Journal of Earth Sciences 40: 651-665. 
2504 De Iuliis, G. and D. Pulerà. 2019. The Dissection of Vertebrates: A Laboratory Manual. Third

2505

2506

2507

2508

2509

2510

2511

2512

2513

2514

2515

2516

2517

2518

2519

2520

2521

2522

2523

2524

2525

2526

2527

2528

2529

2530

2531

2532

2533

2534

2535

2536

2537

2538

2539

2540

2541

2542

2543

2544

2545

2546

2547

2548

2549

Edition. London: Academic Press.

Dodson, P. 1975. Functional and ecological significance of relative growth in Alligator. Zoology 1975: 315-355.

Edwards, G. P., G. J. Webb, S. C. Manolis, and A. Mazanov. 2017. Morphometric analysis of the Australian freshwater crocodile (Crocodylus johnstoni). Australian Journal of Zoology: doi 10.1071/ZO16079.

Erickson, G. M., S. D. Van Kirk, J. Su, M. E. Levenston, W. E. Clear, and D. R. Carter. 1996. Bite-force estimation for Tyrannosaurus rex from tooth-marked bones. Nature 382: 706-708.

Erickson, G. M., A. K. Lappin, and K. A. Vliet. 2003. The ontogeny of bite-force performance in American Alligator (Alligator mississippiensis). Journal of Zoology 260: 317-327.

Erickson, G.M., P. J. Makovicky, P. J. Currie, and M. A. Norell. 2004. Gigantism and comparative life-history parameters of tyrannosaurid dinosaurs. Nature 772-775.

Erickson, G. M. 2005. Assessing dinosaur growth patterns: a microscopic revolution. TRENDS in Ecology and Evolution 20: 677-684.

Erickson, G. M., K. C. Rogers, D. J. Varricchio, M. A. Norell, and X. Xu. 2007. Growth patterns in brooding dinosaurs reveals the timing of sexual maturity in non-avian dinosaurs and genesis of the avian condition. Biology Letters 3: 558-561. Doi: 10.1098/rsbl.2007.0254.

Erickson, G. M., P. M. Gignac, A. K. Lappin, K. A. Vliet, J. D. Brueggen, and G. J. W. Webb. 2013. A comparative analysis of ontogenetic bite-force scaling among Crocodylia. Journal of Zoology 292: 48-55. Doi: 10.1111/jzo.12081.

Ezcurra, M. D. and R. J. Butler. 2015. Post-hatchling ontogeny in the Early Triassic diapsid reptile Proterosuchus fergusi. Journal of Anatomy 226: 387-402.

Foth, C., P. Bona, and J. B. Desojo. 2013. Intraspecific variation in the skull morphology of the black caiman Melanosuchus niger (Alligatoridae, Caimanainae). Acta Zoologica: doi 10.1111/azo.12045.

Foth, C., B. P. Hendrick, and M. D. Ezcurra. 2016. Cranial ontogenetic variation in early saurischians and the role of heterochrony in the diversification of predatory dinosaurs. PeerJ 4:e 1589; DOI 10.7717/peerj.1589.

Foth, C., M. V. Fernandez Blanco, and P. Bona. 2018. Cranial shape variation in jacarean caimanines (Crocodylia, Alligatoroidea) and its implications in the taxonomic status of extinct species: the case of Melanosuchus fisheri. Journal of Morphology 279: 259-273.

Peer) reviewing PDF | (2019:12:43677:1:0:NEW 4 Apr 2020) 
2550

2551

2552

2553

2554

2555

2556

2557

2558

2559

2560

2561

2562

2563

2564

2565

2566

2567

2568

2569

2570

2571

2572

2573

2574

2575

2576

2577

2578

2579

2580

2581

2582

2583

2584

2585

2586

2587

2588

2589

2590

2591

2592

2593

2594

2595
Fowler, D. W. 2017. Revised geochronology, correlation, and dinosaur stratigraphic ranges of the Santonian-Maastrichtian (Late Cretaceous) formations of the Western Interior of North America. PLoS ONE 12: e0188426.

Frederickson, J. A. \& Tumarkin-Deratzian, A. R. 2014. Craniofacial ontogeny in Centrosaurus apertus. PeerJ 2: e252, doi: 10.7717/peerj.252.

Gates, T. A., E. Gorscak, and P. J. Makovicky. 2019. New sharks and other chondrichthyans from the latest Maastrichtian (Late Cretaceous) of North America. Journal of Paleontology 93: 512-530.

Gignac, P. M. and G. M. Erickson. 2014. Ontogenetic changes in dental form and tooth pressures facilitate developmental niche shifts in American alligators. Journal of Zoology. Doi: 10.1111/jzo.12187.

Gignac, P. M. and H. O'Brien. 2016. Suchian feeding success at the interface of ontogeny and macroevolution. Integrative and Comparative Biology 56: 449-458. Doi: 10.1093/icb/icw041.

Gignac, P. M. and G. M. Erickson. 2017. The biomechanics behind extreme osteophagy in Tyrannosaurus rex. Scientific Reports 7: Doi:10.1038/s41598-017-02161-w.

Goloboff, P., J. Farris, and K. Nixon. 2003. TNT: Tree analysis using new technology. Program and documentation available at http://www.lillo.org.ar/phylogeny/tnt/

Goloboff, P. and S. Catalano. 2016. TNT, version 1.5, with a full implementation of phylogenetic morphometrics. Cladistics. DOI: 10.1111/cla.12160.

Griffin, C. T. 2018. Developmental patterns and variation among early theropods. Journal of Anatomy 232: 604-640.

Griffin, C. T. and S. J. Nesbitt. 2016a. The femoral ontogeny and long bone histology of the Middle Triassic (?late Anisian) dinosauriform Asilisaurus kongwe and implications for the growth of early dinosaurs. Journal of Vertebrate Paleontology 26: e1111224. Doi: 10.1080/02724634.2016.1111224.

Griffin, C. T. and S. J. Nesbitt. 2016b. Anomalously high variation in postnatal development is ancestral for dinosaurs but lost in birds. PNAS 113: 14757-14762. Doi: $10.1073 /$ pnas. 1613813113.

Hall, P. M. and Portier, K. M. 1994. Cranial morphometry of New Guinea crocodiles (Crocodylus novaeguineae): ontogenetic variation in relative growth of the skull and an assessment of its utility as a predictor of sex and size of individuals. Herpetological Monographs 8:203-225. 
2596

2597

2598

2599

2600

2601

2602

2603

2604

2605

2606

2607

2608

2609

2610

2611

2612

2613

2614

2615

2616

2617

2618

2619

2620

2621

2622

2623

2624

2625

2626

2627

2628

2629

2630

2631

2632

2633

2634

2635

2636

2637

2638

2639

2640

Harrison, W. F., D. J. Nichols, M. D. Henderson, and R. P. Scherer. 2013. Using pollen, leaves, and paleomagnetism to date a juvenile tyrannosaurid in upper Cretaceous rock. In Tyrannosaurid Paleobiology, pp. 239-250. J. M. Parrish, R. E. Molnar, P. J. Currie, and E. B. Koppelhus (eds). University of Indiana Press.

Henderson, D. M. 2002. The eyes have it: the sizes, shapes, and orientations of theropod orbits as indicators of skull strength and bite force. Journal of Vertebrate Paleontology 22: 766778.

Henderson, D. M. and E. Snively. 2004. Tyrannosaurus en pointe: allometry minimized rotational inertia of large carnivorous dinosaurs. Proceedings of the Royal Society B 271: S57-S60.

Herring, S. W. 1993. Epigenetic and functional influences on skull growth. Pp. 153-206 in J. Hanken and B. K. Hall (eds), The Skull. Volume 1: Development. University of Chicago Press.

Holliday, C. M. 2009. New insights into dinosaur jaw muscle anatomy. The Anatomical Record 292: 1246-1265.

Holliday, C. M. and L. M. Witmer. 2007. Archosaur adductor chamber evolution: integration of musculoskeletal and topological criteria in jaw muscle homology. The Anatomical Record 268: 457-484.

Holliday, C. M., W. R. Porter, K. A. Vliet, and L. M. Witmer. 2019. The frontoparietal fossa and dorsotemporal fenestra of archosaurs and their significance for interpretations of vascular and muscular anatomy in dinosaurs. The Anatomical Record: doi: 10.1002/ar.24218.

Hone, D. W. E., A. A. Farke, and M. J. Wedel. 2016. Ontogeny and the fossil record: what, if anything, is an adult dinosaur? Biology Letters 12: doi 10.1098/rsbl.2015.0947.

Horner, J. R. and K. Padian. 2004. Age and growth dynamics of Tyrannosaurus rex. Proceedings of the Royal Society of London. Series B: Biological Sciences 271: 1875-1880.

Horner, J. R. and M. B. Goodwin. 2006. Major cranial changes during Triceratops ontogeny. Proceedings of the Royal Society of London. Biology 273: 2757-2761.

Horner, J. R. and M. B. Goodwin. 2009. Extreme cranial ontogeny in the Upper Cretaceous dinosaur Pachycephalosaurus. PLoS ONE 4: e7626. Doi: 10.1371/journal.pone.0007626.

Horner, J. R., M. B. Goodwin, and N. Myhrvold. 2011. Dinosaur census reveals abundant Tyrannosaurus rex and rare ontogenetic stages in the Upper Cretaceous Hell Creek Formation (Maastrichtian), Montana, USA. PLoS ONE:doi.org/10.1371/journal.pone.0016574. 
2641 Hurlburt, G. R., R. C. Ridgley, and L. M. Witmer. 2013. Relative size of brain and cerebrum in

2642

2643

2644

2645

2646

2647

2648

2649

2650

2651

2652

2653

2654

2655

2656

2657

2658

2659

2660

2661

2662

2663

2664

2665

2666

2667

2668

2669

2670

2671

2672

2673

2674

2675

2676

2677

2678

2679

2680

2681

2682

2683

2684

2685

2686 tyrannosaurid dinosaurs: an analysis using brain-endocast quantitative relationships in extant alligators. Pp. 135-153 in J. M. Parrish, R. E. Molnar, P. J. Currie, and E. B Koppelhus (eds.), Tyrannosaurid Paleobiology. University of Indiana Press.

Hutchinson, J. R., K. T. Bates, J. Molnar, V. Allen, and P. J. Makovicky. 2011. A computational analysis of limb and body dimensions in Tyrannosaurus rex with implications for locomotion, ontogeny, and growth. PLoS ONE 9: e97055.

Hutton, J. 1989. Movements, home range, dispersal and the separation of size classes in Nile crocodiles. American Zoologist 29: 1033-1049.

IBM Corp. Released 2016. IBM SPSS Statistics for Windows, Verson 24.0. Armonk, NY: IBM Corp.

Larson, P. 2008. Variation and sexual dimorphism in Tyrannosaurus rex. In P. L. Larson and K. Carpenter (eds), Tyrannosaurus rex, the Tyrant King; pp. 103-127. Indiana University Press.

Lee, A. H. and S. Werning. 2008. Sexual maturity in growing dinosaurs does not fit reptilian growth models. Proceedings of the National Association of Science 105: 582-587. Doi: 10.1073/pnas.0708903105.

Leslie, C. E., D. J. Peppe, T. E. Williamson, M. Heizler, M. Jackson, S. C. Atchley, L. Nordt, and B. Standhardt. 2018. Revised age constraints for Late Cretaceous to early Paleocene terrestrial strata from the Dawson Creek section, Big Bend National Park, west Texas. Geological Society of America Bulletin: 10.1130/B31785.1.

Lincoln, R., G. Boxshall, and P. Clark. 1982. A Dictionary of Ecology, Evolution, and Sytematics. Second Edition. Cambridge University Press, London.

Loewen, M. A., R. B. Irmis, J. J. W. Sertich, P. J. Currie, and S. D. Sampson. 2013. Tyrant dinosaur evolution tracks the rise and fall of Late Cretaceous oceans. PLoS ONE 8: e79420. DOI: 10.1371/journal.pone.0079420.

Longrich, N. R. and D. J. Field. 2012. Torosaurus is not Triceratops: ontogeny in chasmosaurine ceratopsids as a case study in dinosaur taxonomy. PLoS ONE 7: e32623.

Maddison, W. P., and D. R. Maddison. 2005. MacClade 4: Analysis of phylogeny and character cvolution. Version 4.08a.

Meers, M. B. 2002. Maximum bite force and prey size of Tyrannosaurus rex and their relationships to the inference of feeding behavior. Historical Biology 16: 1-12.

Molnar, R. E. 1980. An albertosaur from the Hell Creek Formation of Montana. Journal of Paleontology 54: 102-108. 
2687

2688

2689

2690

2691

2692

2693

2694

2695

2696

2697

2698

2699

2700

2701

2702

2703

2704

2705

2706

2707

2708

2709

2710

2711

2712

2713

2714

2715

2716

2717

2718

2719

2720

2721

2722

2723

2724

2725

2726

2727

2728

2729

2730

2731

2732

Molnar, R. E. 1990. The cranial morphology of Tyrannosaurus rex. Palaeontographica Abt. A 217: 137-176.

Molnar, R. E. 2013. A comparative analysis of reconstructed jaw musculature and mechanics of some large theropods. Pp. 177-193 in J. M. Parrish, R. E. Molnar, P. J. Currie, and E. B Koppelhus (eds.), Tyrannosaurid Paleobiology. University of Indiana Press.

Monteiro, L. R. and M. Soares. 1997. Allometric analysis of the ontogenetic variation and evolution of the skull in Caiman Spix, 1825 (Crocodylia: Alligatoridae). Herpetologica 53: 62-69.

Monteiro, L. R., M. J. Cavalcanti, and H. J. S. Sommer III. 1997. Comparative ontogenetic shape changes in the skull of Caiman species (Crocodylia, Alligatoridae). Journal of Morphology 231: 53-62.

Moss, M. L. 1957. Experimental alteration of sutural area morphology. The anatomical record 127: 569-589.

O’Connor, J., G. M. Erickson, M. Norell, A. M. Bailleul, H. Hu, and Z. Zhou. 2018. Medullary bone in an early Cretaceous enantiornithine bird and discussion regarding its identification in fossils. Nature Communications. Doi: 10.1038/s41467-018-07621-z.

Osborn, H. F. 1905. Tyrannosaurus and other Cretaceous carnivorous dinosaurs. Bulletin of the American Museum of Natural History 21: 259-265.

Osborn, H. F. 1906. Tyrannosaurus, Upper Cretaceous carnivorous dinosaur: (second communication). Bulletin of the American Museum of Natural History 22: 281-296.

Padian, K. 1995. Form versus function: the evolution of a dialectic. Pp. 264-277 in J. J. Thomason (ed.), Functional Morphology in Vertebrate Paleontology. University of Cambridge Press.

Padian, K. and J. R. Horner. 2011. The definition of sexual selection and its implications for dinosaurian biology. Journal of Zoology 283: 23-27.

Paul, G. S. 1988. Predatory Dinosaurs of the World: A Complete Illustrated Guide. Simon and Schuster, New York.

Persons, W. S., P. J. Currie, and G. M. Erickson. 2019. An older and exceptionally large adult specimen of Tyrannosaurus rex. The Anatomical Record doi: 10.1002/ar.24118.

Piras, P., P. Colangelo, D. C. Adams, A. D. Buscalioni, J. Cubo, T. Kotsakis, C. Meloro, and P. Raia. 2010. The Gavialis-Tomistoma debate: the contribution of skull ontogenetic allometry and growth trajectories to the study of crocodylian relationships. Evolution \& Development 12: 568-579.

Peer) reviewing PDF | (2019:12:43677:1:0:NEW 4 Apr 2020) 
2733

2734

2735

2736

2737

2738

2739

2740

2741

2742

2743

2744

2745

2746

2747

2748

2749

2750

2751

2752

2753

2754

2755

2756

2757

2758

2759

2760

2761

2762

2763

2764

2765

2766

2767

2768

2769

2770

2771

2772

2773

2774

2775

2776

2777

2778

Platt, S. G., T. R. Rainwater, J. B. Thorbjarnarson, and D. Martin. 2011. Size estimation, morphometrics, sex ratio, sexual size dimorphism, and biomass of Crocodylus acutus in the coastal zone of Belize. Salamandra 47: 179-192.

Rauhut, O. W. M. 2003. The interrelationships and evolution of basal theropod dinosaurs. Special Papers in Palaeontology 69: 1- 213.

Rayfield, E. J. 2004. Cranial mechanics and feeding in Tyrannosaurus rex. Proceedings of the Royal Society of London Series B 271: 1451-1459.

Rose, C. S. and J. O. Reiss. 1993. Metamorphosis and the vertebrate skull: ontogenetic patterns and developmental mechanisms. In The Skull. Volume 1: Development, H. Hanken and B. K. Hall (eds). University of Chicago Press.

Schmerge, J. D. and D. M. Rothschild. 2016a. Distribution of the dentary groove of theropod dinosaurs: implications for theropod phylogeny and the validity of the genus Nanotyrannus Bakker et al., 1988. Cretaceous research 61: 26-33.

Schmerge, J. D. and D. M. Rothschild. 2016b. When a groove is not a groove: clarification of the appearance of the dentary groove in tyrannosauroid theropods and the distinction between Nanotyrannus and Tyrannosaurus. Reply to comment on: "Distribution of the dentary groove of theropod dinosaurs: implications for theropod phylogeny and the validity of the genus Nanotyrannus Bakker et al., 1988". Cretaceous Research 65: 238243.

Schweitzer, M. H, J. L. Wittmeyer, and J. R. Horner. 2005. Gender-specific reproductive tissue in ratites and Tyrannosaurus rex. Science 308: 1456-1460.

Sebens, K. P. 1987. The ecology of indeterminate growth in animals. Annual Review of Ecology and Systematics 18: 371-407.

Snively, E. and A. P. Russell. 2003. Kinematic model of tyrannosaurid (Dinosauria: Theropoda) arctometatarsus function. Journal of Morphology 255: 215-227.

Snively, E., D. M. Henderson, and D. S. Phillips. 2006. Fused and vaulted nasals of tyrannosaurid dinosaurs: implications for cranial strength and feeding mechanics. Acta Palaeontologica Polonica 51: 435-454.

Snively, E. H. O’Brien, D. M. Henderson, H. Mallison, L. Surring, M. E. Burns, T. R. Holtz Jr, A. P. Russell, L. M. Witmer, P. J. Currie, S. A. Hartman, and J. R. Cotton. 2019. Lower rotational inertia and larger leg muscles indicate more rapid turns in tyrannosaurids than in other large theropods. PeerJ 7: e6432.

Swofford, D. L. 2002. PAUP*. Phylogenetic Analysis Using Parsimony (*and Other Methods). Version 4. Sinauer Associates, Sunderland, Massachusetts.

Peer] reviewing PDF | (2019:12:43677:1:0:NEW 4 Apr 2020) 
2779

2780

2781

2782

2783

2784

2785

2786

2787

2788

2789

2790

2791

2792

2793

2794

2795

2796

2797

2798

2799

2800

2801

2802

2803

2804

2805

2806

2807

2808

2809

2810

2811

2812

2813

2814

2815

2816

2817

2818

2819

2820

2821

2822

2823

2824
Taylor, P., F. Li, A. Holland, M. Martin, and A. E. Rosenblatt. 2016. Growth rates of black caiman (Melanosuchus niger) in the Rupununi region of Guyana. Amphibia-Reptilia 27: 9-14.

Therrien, F., D. Henderson, and C. B. Ruff. 2005. Bite me: biomechanical models of theropod mandibles and implications for feeding behavior. In K. Carpenter (ed.), The Carnivorous Dinosaurs, Indiana University Press, Bloomington, pp. 179-237.

Tucker, A. D., C. J. Limpus, K. R. McDonald, and H. I. McCallum. 2006. Growth dynamics of freshwater crocodiles (Crocodylus johnstoni) in the Lynd River, Queensland. Australian Journal of Zoology 54: 409-415.

Tumarkin-Deratzian, A. R., D. R. Vann, and P. Dodson. 2006. Bone surface texture as an ontogenetic indicator in long bones of the Canada goose Branta canadensis (Anseriformes: Anatidae). Zoological Journal of the Linnean Society 148: 133-168.

Van de Graaff, K. M. 1998. Human Anatomy, Fifth Edition. Boston: WCB/McGraw-Hill.

Werneburg, I., D. Esteve-Altava, J. Bruno, M. T. Ladeira, and Rui Diogo. 2019. Unique skull network complexity of Tyrannosaurus rex among land vertebrate. Scientific Reports 9: 1520.

Whitlock, M. C. and D. Schluter. 2015. The Analysis of Biological Data. Second Edition. Roberts and Company Publishers.Greenwood Village, Colorado. 818 pp.

Wilkinson, P. M., T. R. Rainwater, A. R. Woodward, E. H. Leone, and C. Carter. 2016. Determinate growth and reproductive lifespan in the American alligator (Alligator mississippiensis): evidence from long-term recaptures. Copeia 104: 843-852.

Wilkinson, P. M. and W. E. Rhodes. 1997. Growth rates of American alligators in coastal South Carolina. The Journal of Wildlife Management. 61: 397-402.

Witmer, L. M. 1997. The evolution of the antorbital cavity of archosaurs: a study in soft-tissue reconstruction in the fossil record with and analysis of the function of pneumaticity. Journal of Vertebrate Paleontology 17, sup 1: 1-76. DOI: 10.1080/02724634.1997.10011027.

Witmer, L.M. and R.C. Ridgley. 2009. New insights into the brain, braincase and ear region of tyrannosaurs (Dinosauria, Theropoda) with implications for sensory organization and behavior. Anatomical Record 292: 1266-1296.

Witmer, L.M. and R.C. Ridgley. 2010. The Cleveland tyrannosaur skull (Nanotyrannus or Tyrannosaurus): new findings based on CT scanning, with special reference to the braincase. Kirtlandia 57: 61-81. 
2825

2826

2827

2828

2829

2830

2831

2832

2833

2834

2835

Wolff, E. D. S., S. W. Salisbury, J. R. Horner, and D. J. Varricchio. 2009. Common avian infection plagued the tyrant dinosaurs. PLoS ONE 4: e7288. Doi: 10.1371/journal.pone.0007288.

Woodward, H., K. Tremaine, S. A. Williams, L. E. Zanno, J. R. Horner, and N. Myhrvold. 2020. Growing up Tyrannosaurus rex: osteohistology refutes the pygmy "Nanotyrannus" and supports ontogenetic niche partitioning in juvenile Tyrannosaurus. Science Advances 6.

Wu, X. B., H. Xue, L. S. Wu, J. L. Zhu, and R. P. Wang. 2006. Regression analysis between body and head measurements of Chinese alligators (Alligator sinensis) in the captive population. Animal Biodiversity and Conservation 29: 65-71. 


\section{Figure 1}

Results of the cladistic analysis of 1,850 characters among 44 specimens of Tyrannosaurus rex.

(A) Strict consensus of 50 MPTs showing the recovery of three primary growth stages separated by the specimen BMRP 2002.4.1. (B) The single ontogram recovered after the exclusion of wildcard specimens, reducing the number of OTUs to 31 . Numbers to the left of the internodes are bootstrap and jackknife values, respectively; numbers to the right are Bremer decay indices. Asterisk indicates the type specimen. Ellipses enclose the regions of polytomies produced by the wildcard specimens, which are listed in the lower right hand corner of the corresponding ellipse. Note that the ellipses are limited to one side or the other relative to BMRP 2002.4.1, which corresponds to the topology of the strict consensus tree. 


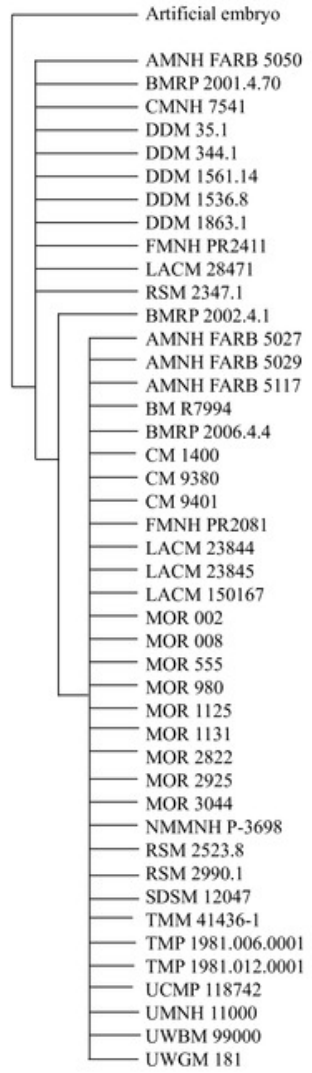

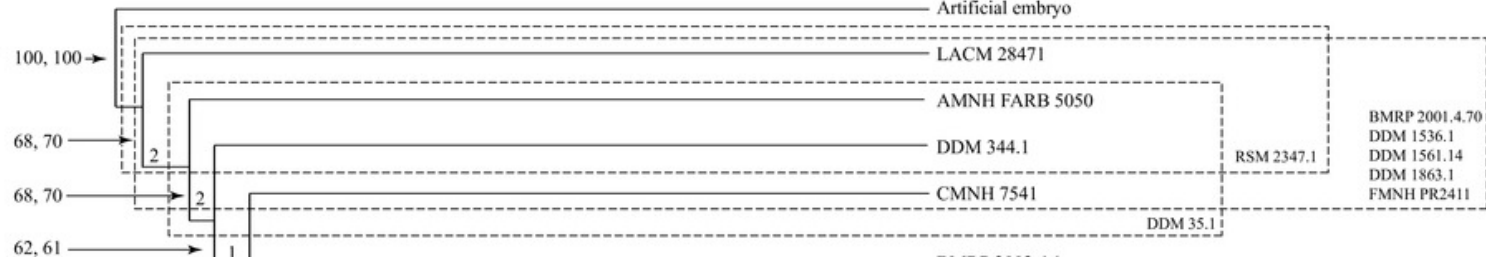

$62,61 \longrightarrow \mathrm{I}$ 95, 95

56.6

56,69

$\mathrm{n} / \mathrm{a}, 63$

LACM 23845

MOR 3044

$\longrightarrow 4-5$ MOR 1125

TMP 1981.006.0001

LACM 150167

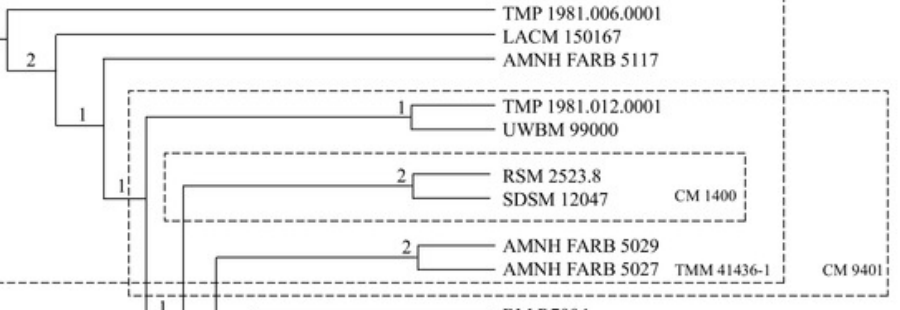

BM R7994

1 NMMNH P-3698

$12 \sqrt{3}$ MOR 113

3 - MOR 980

1

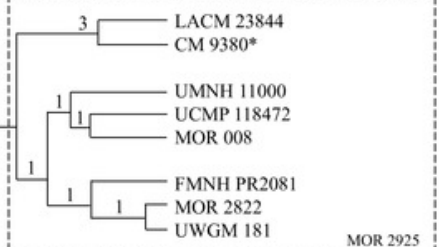




\section{Figure 2}

Ontogram of Tyrannosaurus rex showing growth stages, synontomorphies, individual variation, individual specimens, and chronological ages.

Arrowhead points to the most mature specimen and the direction of the entire ontogenetic axis; i.e., the least mature specimen is at the lower left whereas the most mature specimen is at the upper right. Asterisk indicates the type specimen. Individual variation occurs as progressions until young adulthood, where reversals are first seen. The maximum amount of change occurs at growth stages 5 and 6 , which corresponds to the transition from a long and low skull and jaws to a deep and stout skull frame; this event, marked by the concentration of an extreme number of changes, is evidence that the ontogeny of $T$. rex is metamorphic (sensu Rose and Reiss, 1993). Each circle represents a numbered growth stage; these numbers do not correspond to those seen in Fig. 12. The star at growth stage 7 marks the $\sim 3,000 \mathrm{~kg}$ threshold that separates $\mathrm{T}$. rex from its closest, but smaller, relatives. Color key: red, small juveniles; orange, large juveniles; yellow, subadults; green, young adults; blue, adults; violet, senescent adults. See text for definition of growth categories. 
Key for synontomorphies (internodes)

and individual variation (branches)

— \# progressions (e.g., $0 \Rightarrow 1$ )

$\smile$ \# reversals (e.g., $1 \Rightarrow 0$ )

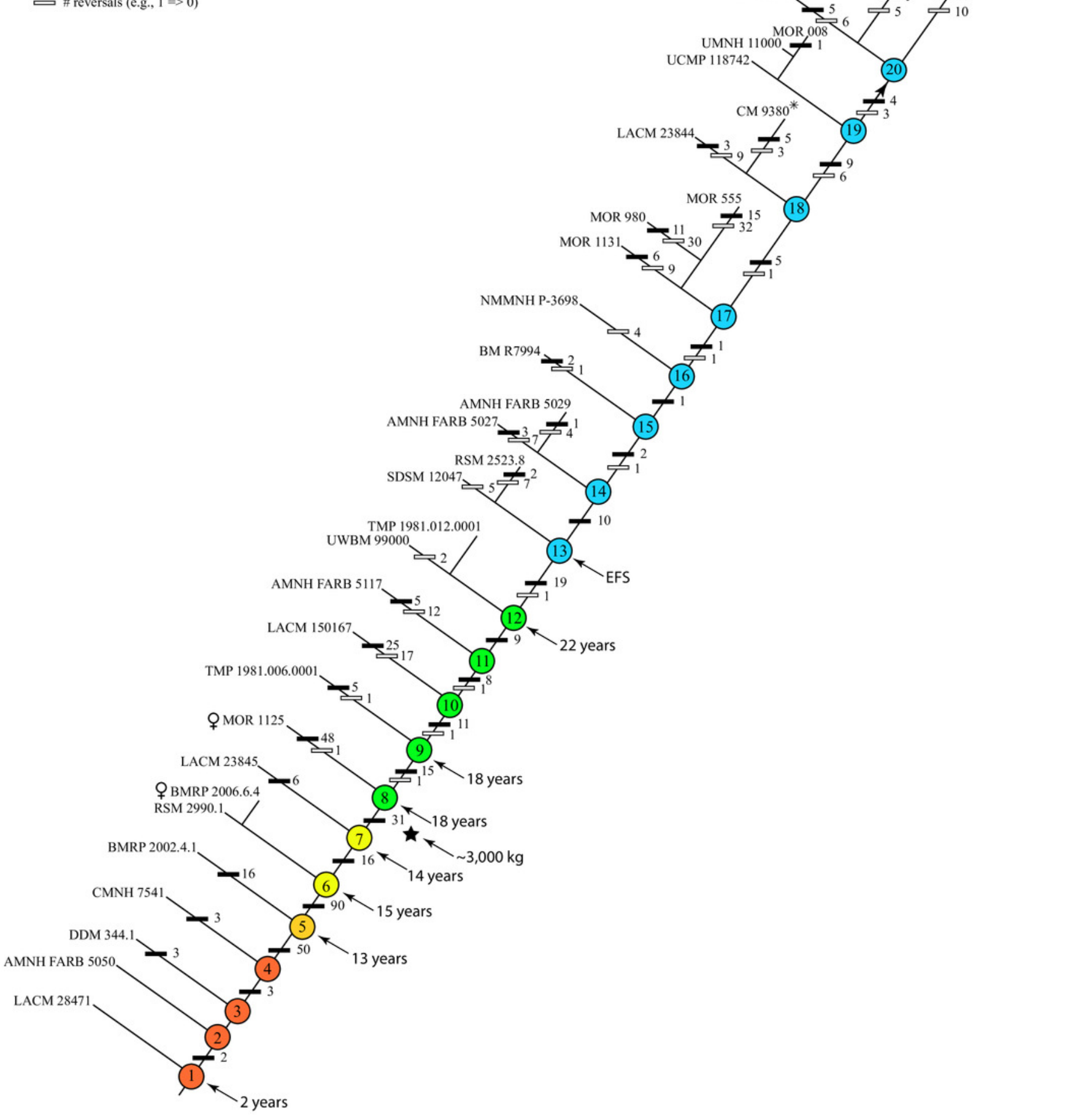




\section{Figure 3}

Scatterplot showing the noncongruence in Tyrannosaurus rex between the completeness of specimens (i.e., number of characters scored) and the number of synontomorphies at each corresponding node.

Per cent completeness (decreasing away from the origin) and the number of synontomorphies supporting the corresponding node (decreasing away from the origin) have been converted to ranks. A Spearman correlation test on these data results in a nonsignificant correlation coefficient; ergo, the number of synontomorphies at an internode is not an artifact of specimen completeness.

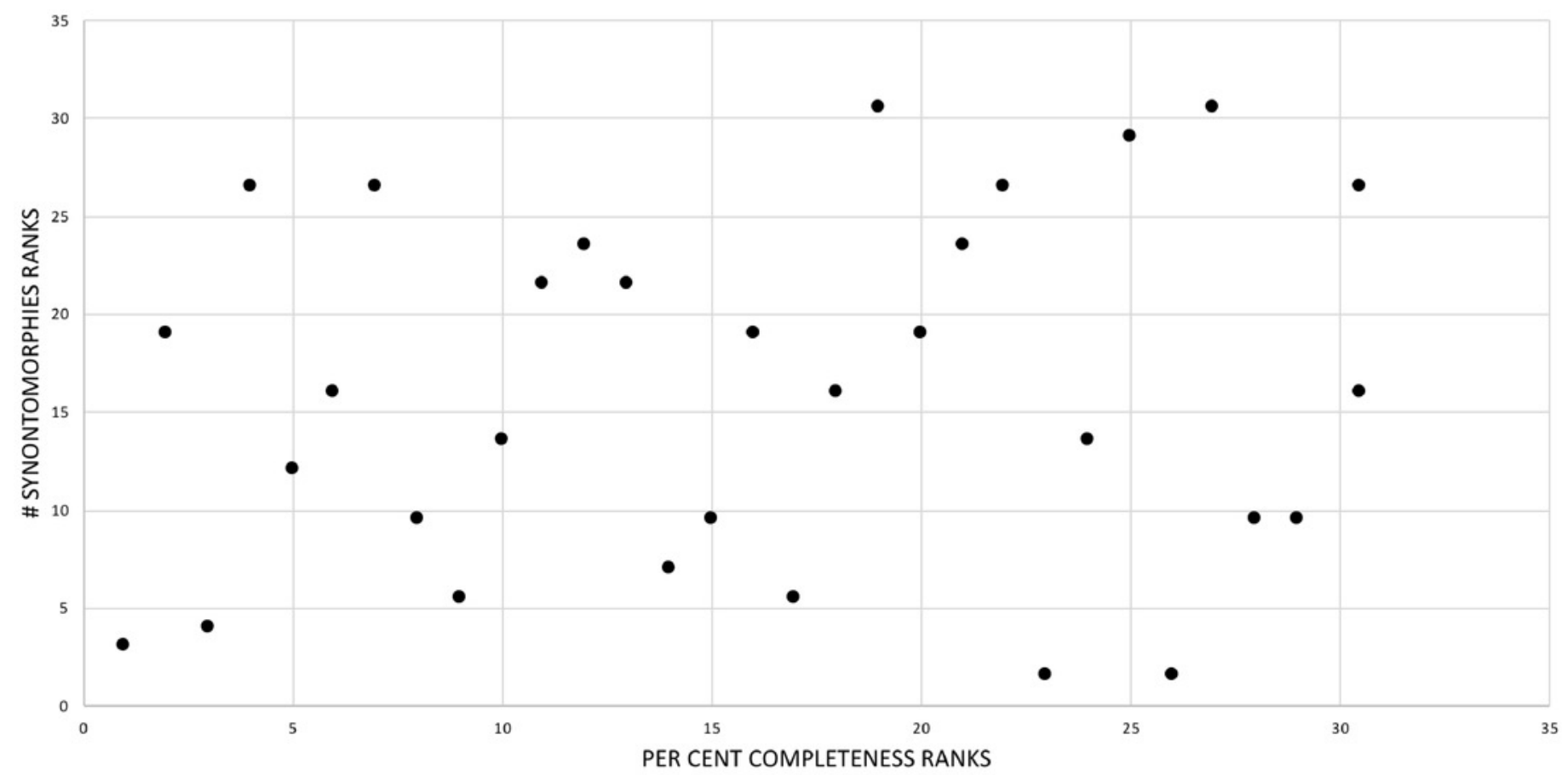




\section{Figure 4}

Frequency distribution of unambiguously optimized synontomorphies during the growth of Tyrannosaurus rex.

Growth stages (corresponding to the numbered nodes of the ontogram in Figure 2) are along the $x$-axis and the number of changes are along the $y$-axis. The greatest number of changes are seen in the transition from large juvenile to subadult, or, from growth stage 5 to 6 ; the high concentration of change between these growth categories is evidence that $T$. rex ontogeny is metamorphic (sensu Rose and Reiss, 1993).

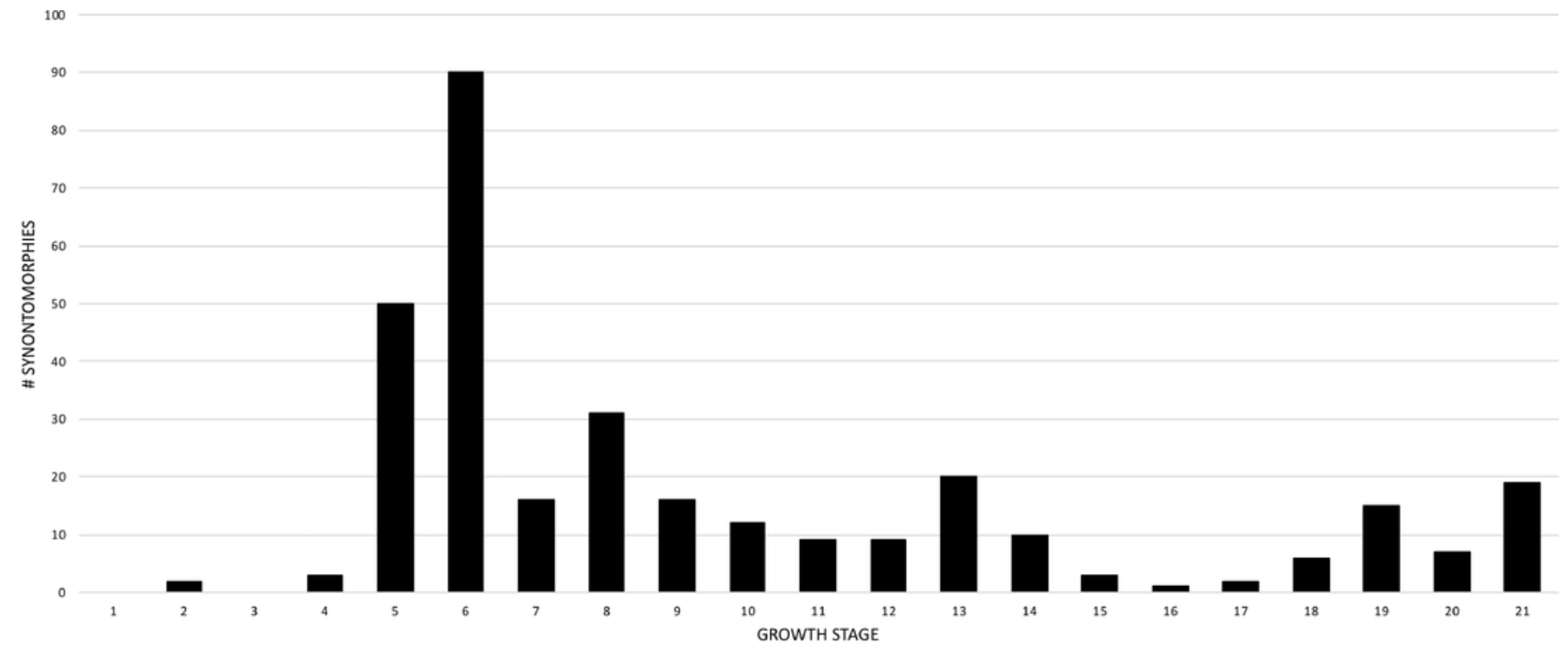




\section{Figure 5}

Comparison of the frequency distributions of phylogenetic and nonphylogenetic synontomorphies in the ontogeny of Tyrannosaurus rex.

Growth stage is along the $\mathrm{x}$-axis (corresponding to the numbered nodes of the ontogram in Figure 2) and number of synontomorphies is along the $y$-axis. Phylogenetic characters are in solid bars; nonphylogenetic characters are in hollow bars. The frequency distributions of both sets of data follow the same general pattern, aside from the flatter distribution of the phylogenetic synontomorphies relative to the nonphylogenetic synontomorphies and the reversed pattern seen at growth stages 7 and 8 . Both types of changes occur throughout the lifespan of $T$. rex, indicating that ontogeny is not strictly congruent with phylogeny.

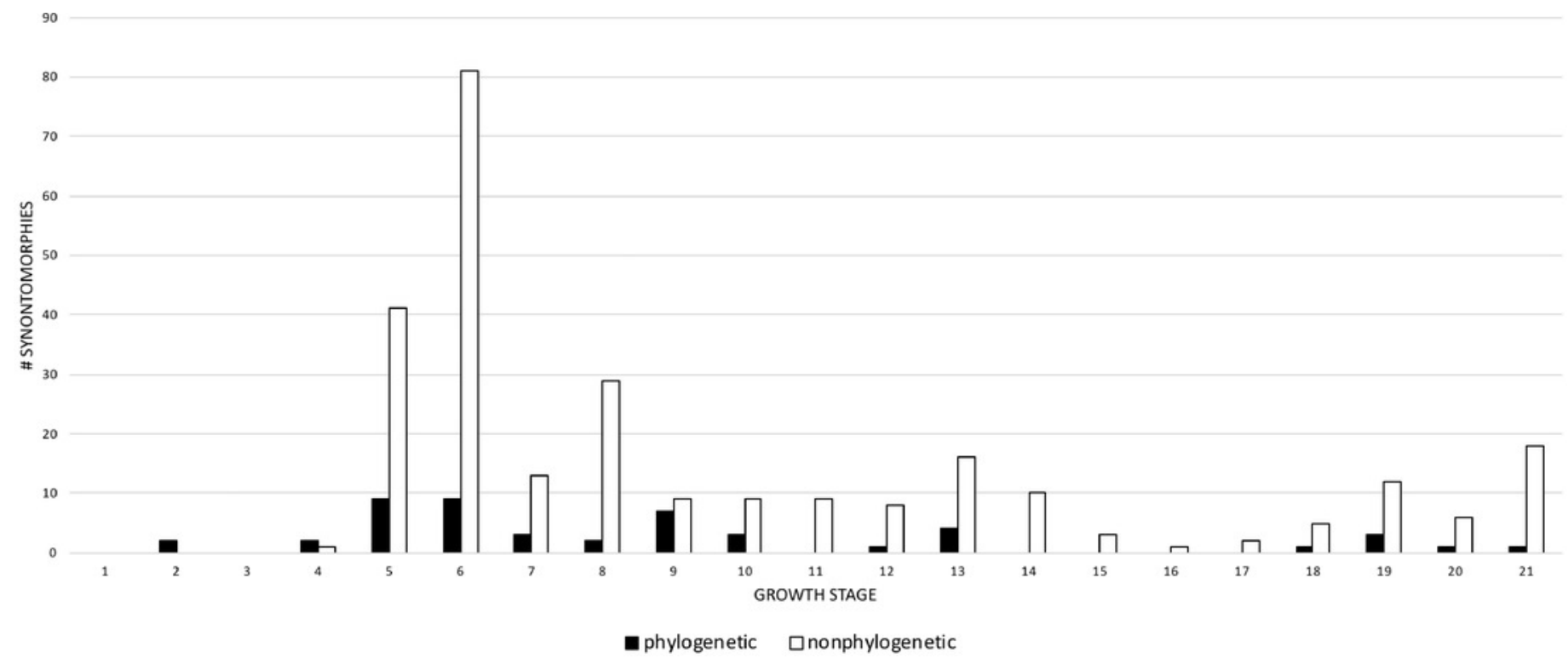




\section{Figure 6}

Comparison of the frequency distributions of cranial and postcranial changes in the growth series of Tyrannosaurus rex.

The growth stages are along the $\mathrm{x}$-axis (corresponding to the numbered nodes of the ontogram in Figure 2) and the y-axis corresponds to the number of synontomorphies. Cranial changes are shown in solid bars; postcranial chanages are shown in hollow bars. Cranial and postcranial changes tend to follow the same overall pattern although postcranial changes are exceeded by cranial changes, except at growth stages 7,15 , and 16 . The relatively late occurrence of postcranial changes (at growth stage 6 ) is an artifact of the absence of postcranial material among the least mature specimens in the sample.

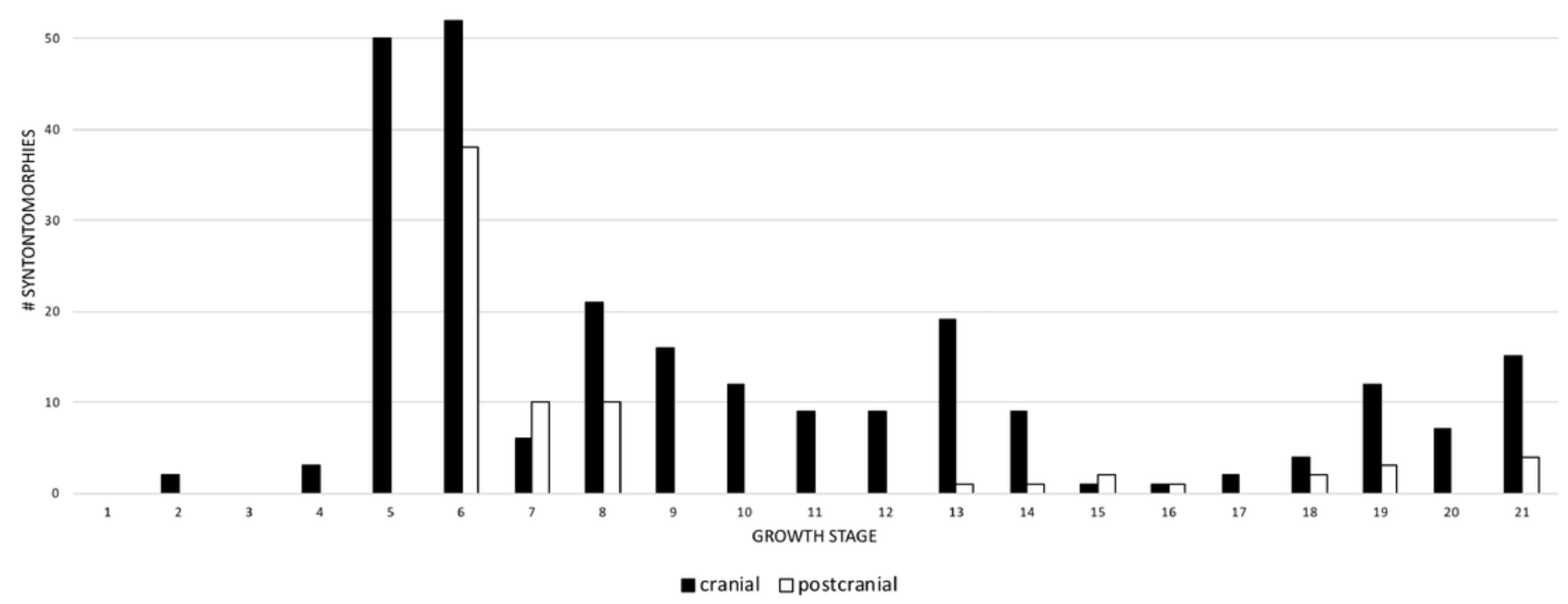




\section{Figure 7}

Comparison of the frequency distribution of synontomorphies of the cranium with that of the mandibular ramus in the ontogeny of Tyrannosaurus rex.

Growth stages are along the $\mathrm{x}$-axis (corresponding to the numbered nodes of the ontogram in Figure 2) and the $y$-axis corresponds to the number of synontomorphies. Skull changes are shown with solid bars; mandible changes are shown with hollow bars. Although a greater number of changes is seen in the cranium than in the mandibular ramus, the lower jaw completes its early phase of changes (stage 5) before the cranium (stage 6). Thereafter, the pattern of mandibular changes is generally congruent with the cranium.

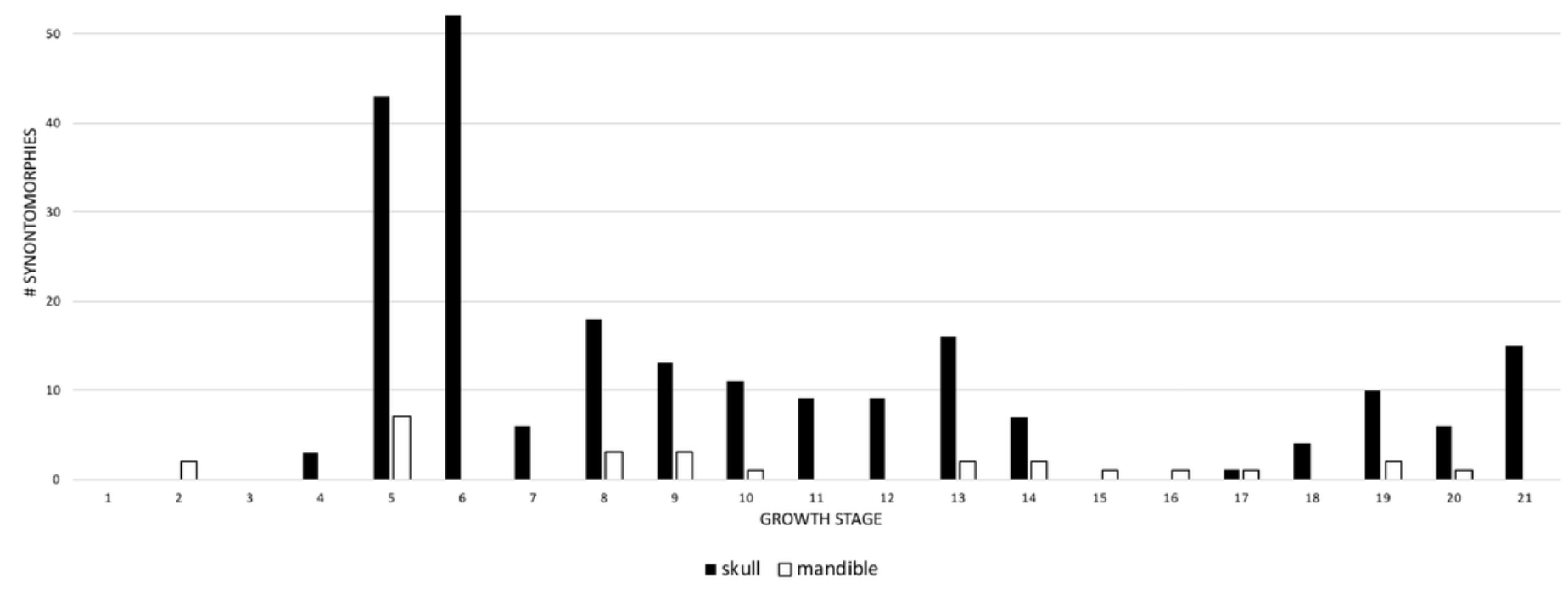


Figure 8

The frequency distribution of synontomorphies by cephalic pneumatic system in the growth series of Tyrannosaurus rex.

Growth stages are along the x-axis (corresponding to the numbered nodes of the ontogram in Figure 2) and the $y$-axis corresponds to the number of synontomorphies. Changes to the antorbital sinus system are dominant over others and are sustained though growth, in contrast to the other systems that occur in adulthood and are transient in occurrence. Abbreviations: aosin, antorbital sinus system; pharyn, pharyngeal sinus system; subcon, subcondylar sinus system; tympcav, tympanic cavity.

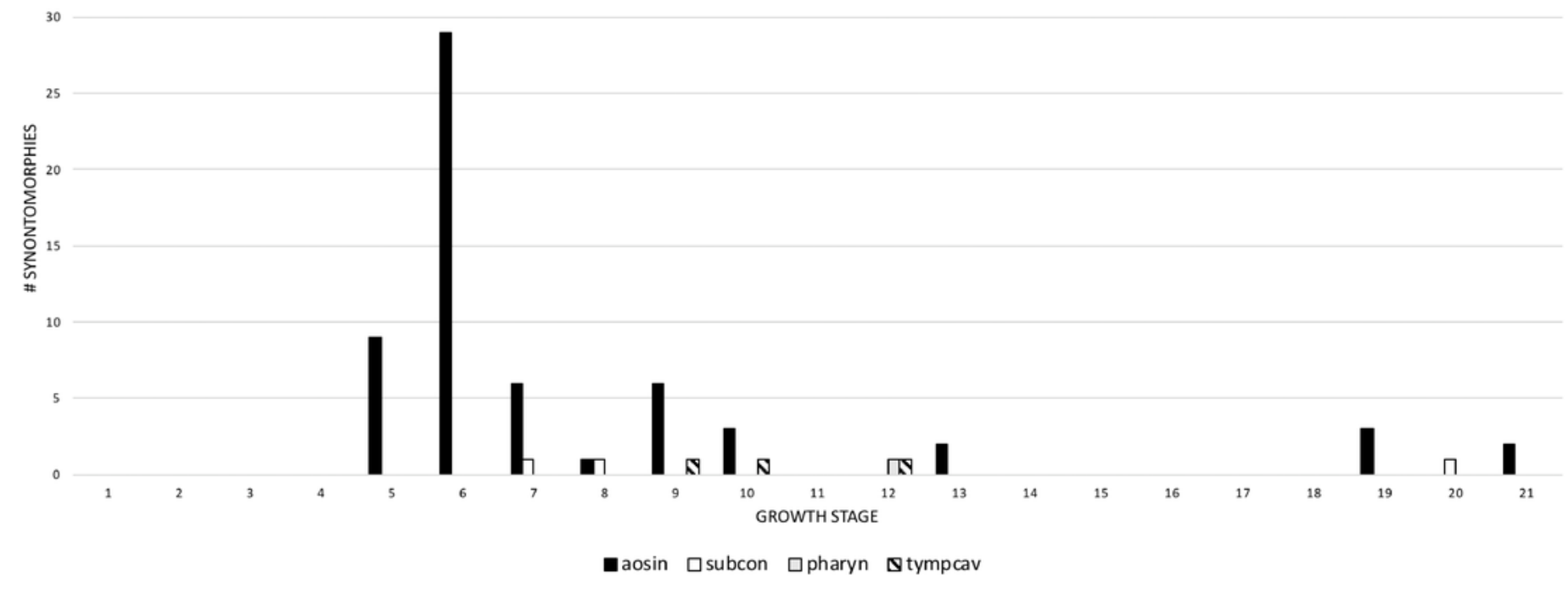




\section{Figure 9}

The frequency distribution of synontomorphies by apneumatic anatomical domain in the growth series of Tyrannosaurus rex.

Growth stages are along the x-axis (corresponding to the numbered nodes of the ontogram in Figure 2) and the $y$-axis corresponds to the number of synontomorphies. Changes to the skull frame are dominant over others and all are sustained throughout growth, aside from the dentition and cervical occiput. Abbreviations: crv occ, cervical occiput; dntn, dentition; dtfo, dorsotemporal fossa; jnt srfc, joint surfaces; mscl scrs, muscle scars; nrvsc, neurovasculature; sbct srfc, subcutaneous surface; skl frm, skull frame.

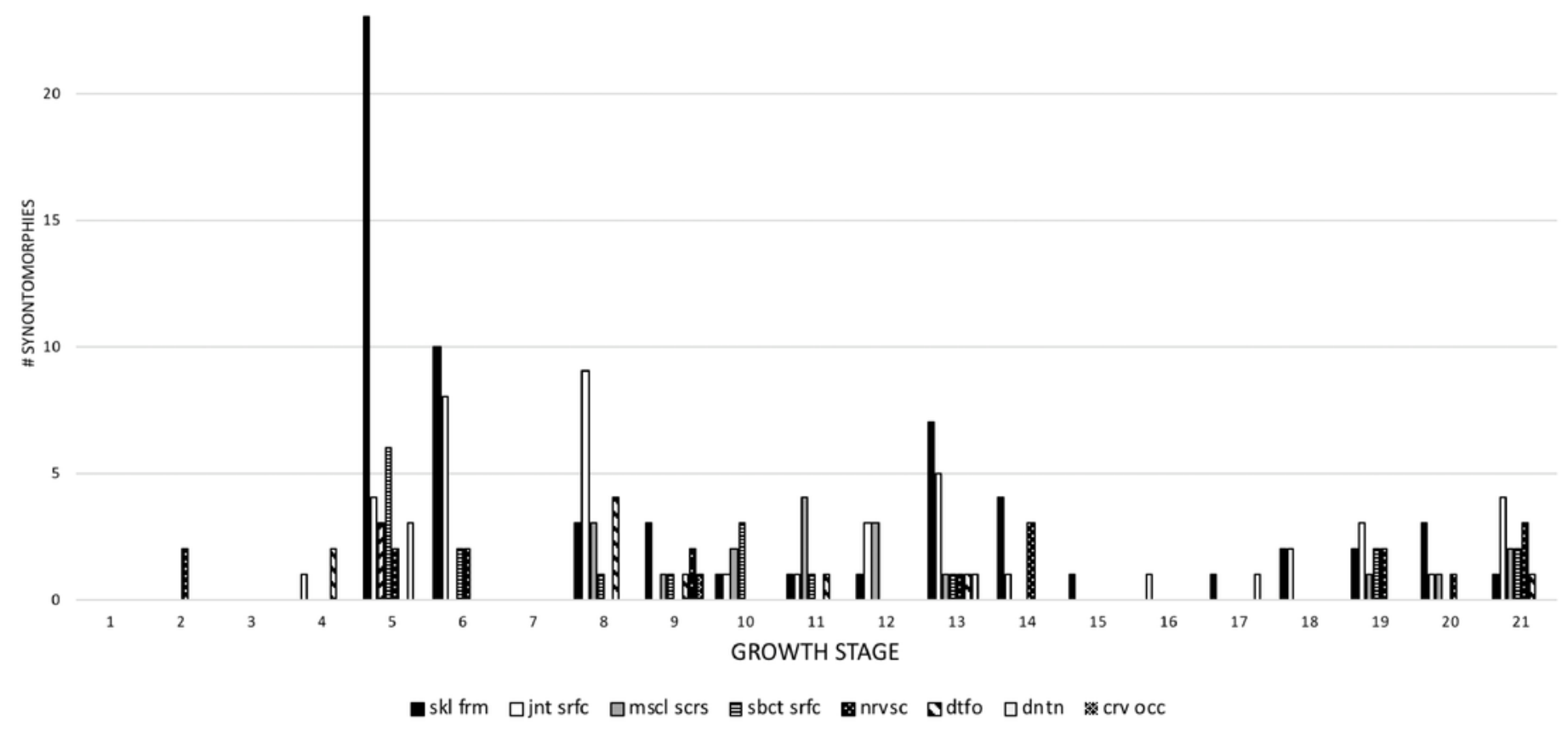




\section{Figure 10}

The frequency distribution of postcranial synontomorphies in the growth series of Tyrannosaurus rex.

Growth stages are along the x-axis (corresponding to the numbered nodes of the ontogram in Figure 2) and the y-axis corresponds to the number of synontomorphies. Changes to the appendicular skeleton dominate in the transition between juvenile and subadult, whereas changes to the pelvic girdle and axial skeleton occur late in adulthood.

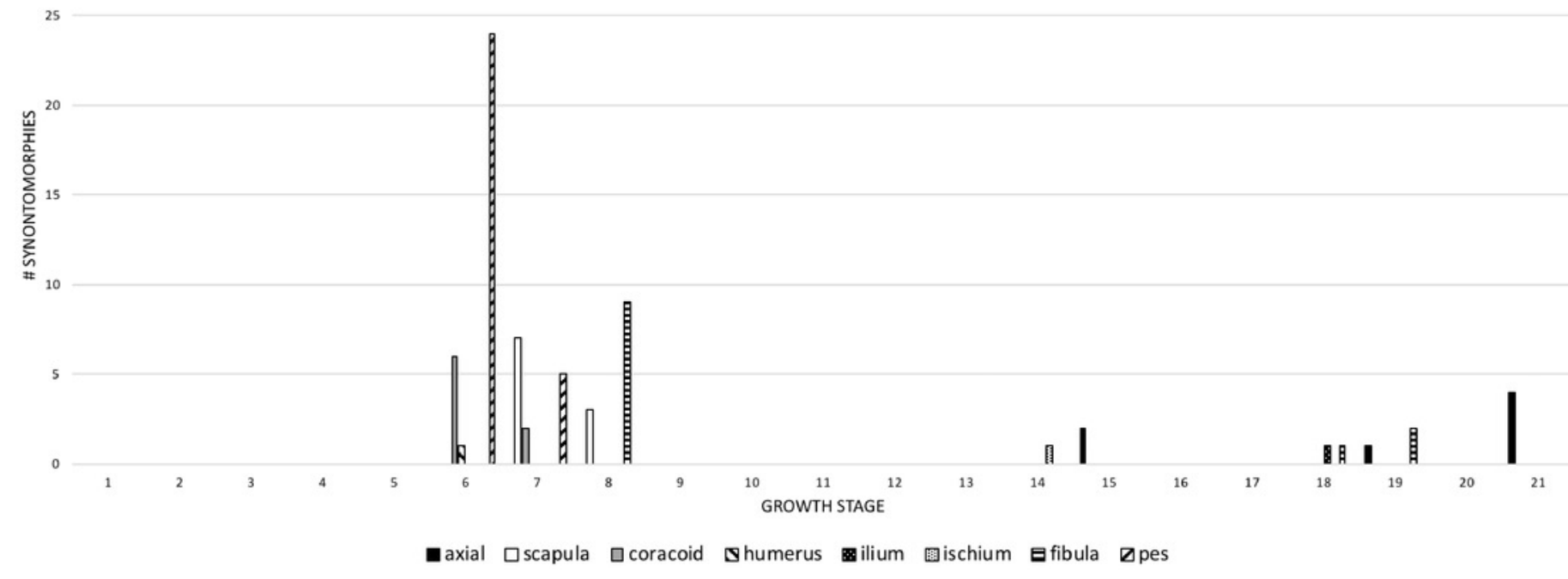


Figure 11

The frequency distribution of changes to the craniomandibular functional modules (sensu Werneburg et al., 2019) in the growth series of Tyrannosaurus rex.

Growth stages are along the $\mathrm{x}$-axis (corresponding to the numbered nodes of the ontogram in Figure 2) and the y-axis corresponds to the number of synontomorphies. The onset of the changes to the skull roof, snout, mandibular ramus, and suspensorium modules occur early in growth, whereas the onset of changes to the parietal and braincase occur in adulthood.

Changes continue throughout growth in all domains, aside from those to the parietal that cease at growth stage 14 .

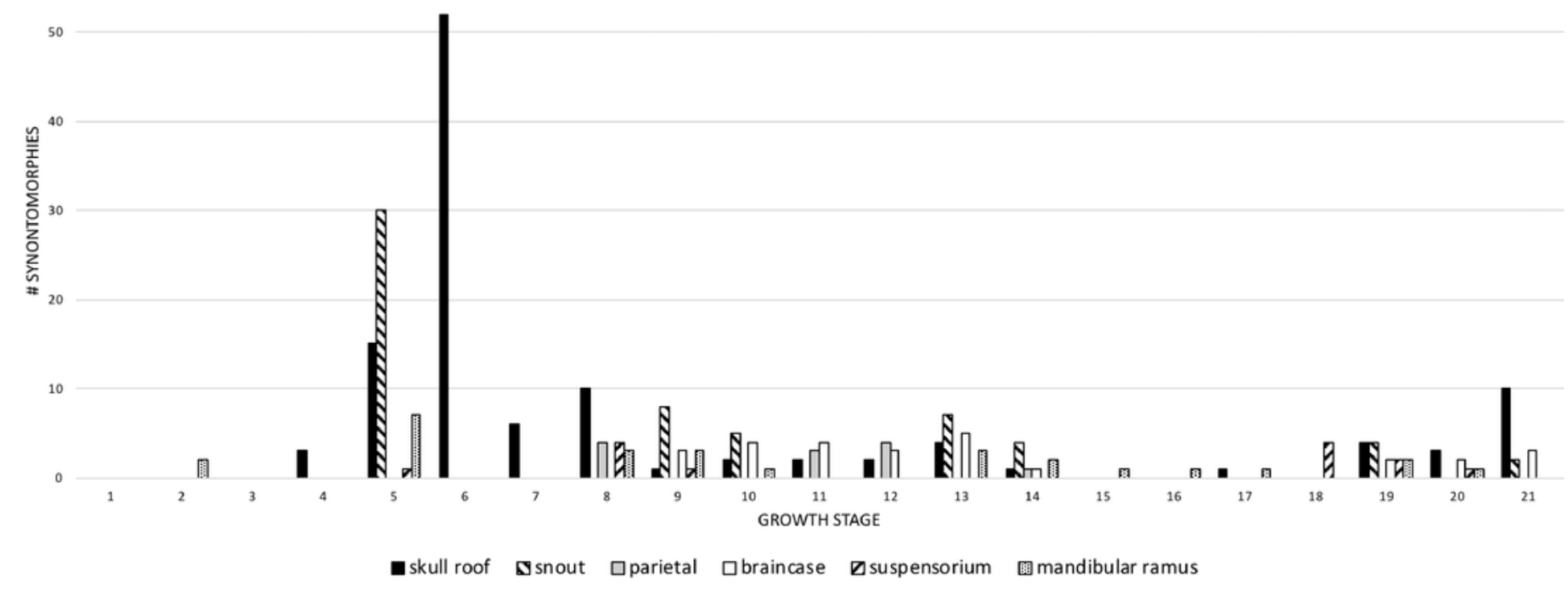




\section{Figure 12}

Tyrannosaurus rex ontogram, chronological age, and mass mapped onto the growth curve of Erickson et al. (2004).

Ranges of growth categories are indicated across the top. Each circle represents an individual specimen; the vertical columns of circles indicate multiple specimens in a single growth stage; the horizontal position of the white circles does not imply a specific chronological age. For ease of interpretation, and to accommodate missing mass estimates, the position of the circles do not correspond with the scale of the $y$-axis. The grey circles indicate histologically aged specimens that are connected to the $\mathrm{x}$-axis by dashed lines for ease of interpretation. The star represents the $\sim 3,000 \mathrm{~kg}$ threshold that separates $T$. rex from its closest, but smaller, relatives. Alternating grey and white bars in the background block out the growth categories and their blurred edges reflect the imprecision of their boundaries. The height of each black vertical bar corresponds to the number of synontomorphies in each growth stage, which ranges from 1 to 90 characters. Asterisk indicates the type specimen of $T$. rex. Female symbols indicate BMRP 2006.6.4 and MOR 1125 , the only unambiguous female specimens in the data set. Skull illustrations are to scale, with that of the adult set to $1.3 \mathrm{~m}$. From left to right the skulls are: CMNH 7541, BMRP 2002.4.1, and AMNH FARB 5027. The large juvenile BMRP 2002.4.1 is in dorsal view to show the early occurrence of the narrow snout and wide temporal region that characterizes $T$. rex to the exclusion of other tyrannosaurids, which is ontogenetically incongruent with its lateoccurring (i.e., autapomorphic) phylogenetic homologue; see text for discussion. Major life history events are indicated, including the onset of sexual maturity and somatic maturity, and the earliest occurrence of histologic adulthood. Suspensorium in CMNH 7541 is reconstructed after BMRP 2002.4.1. Abbreviation: EFS, External Fundamental System. Specimens: 1, LACM 28471; 2, AMNH FARB 5050; 3, DDM 344.1; 4, CMNH 7541; 5, BMRP 
2002.4.1; 6, RSM 2990.1; 7, BMRP 2006.6.4; 8, LACM 23845; 9, MOR 1125; 10, TMP 1981.006.0001; 11, LACM 150167; 12, AMNH FARB 5117; 13, UWBM 99000; 14, TMP 1981.012.0001; 15, RSM 2523.8; 16, SDSM 12047; 17, AMNH FARB 5027; 18, AMNH FARB 5029; 19, BM R7994; 20, NMMNH P-3698; 21, MOR 1131; 22, MOR 980; 23, MOR 555; 24, LACM 23844; 25, CM 9380; 26, UCMP 118742; 27, MOR 008; 28, UMNH 11000; 29, MOR 2822; 30, UWGM 181; 31, FMNH PR2081.

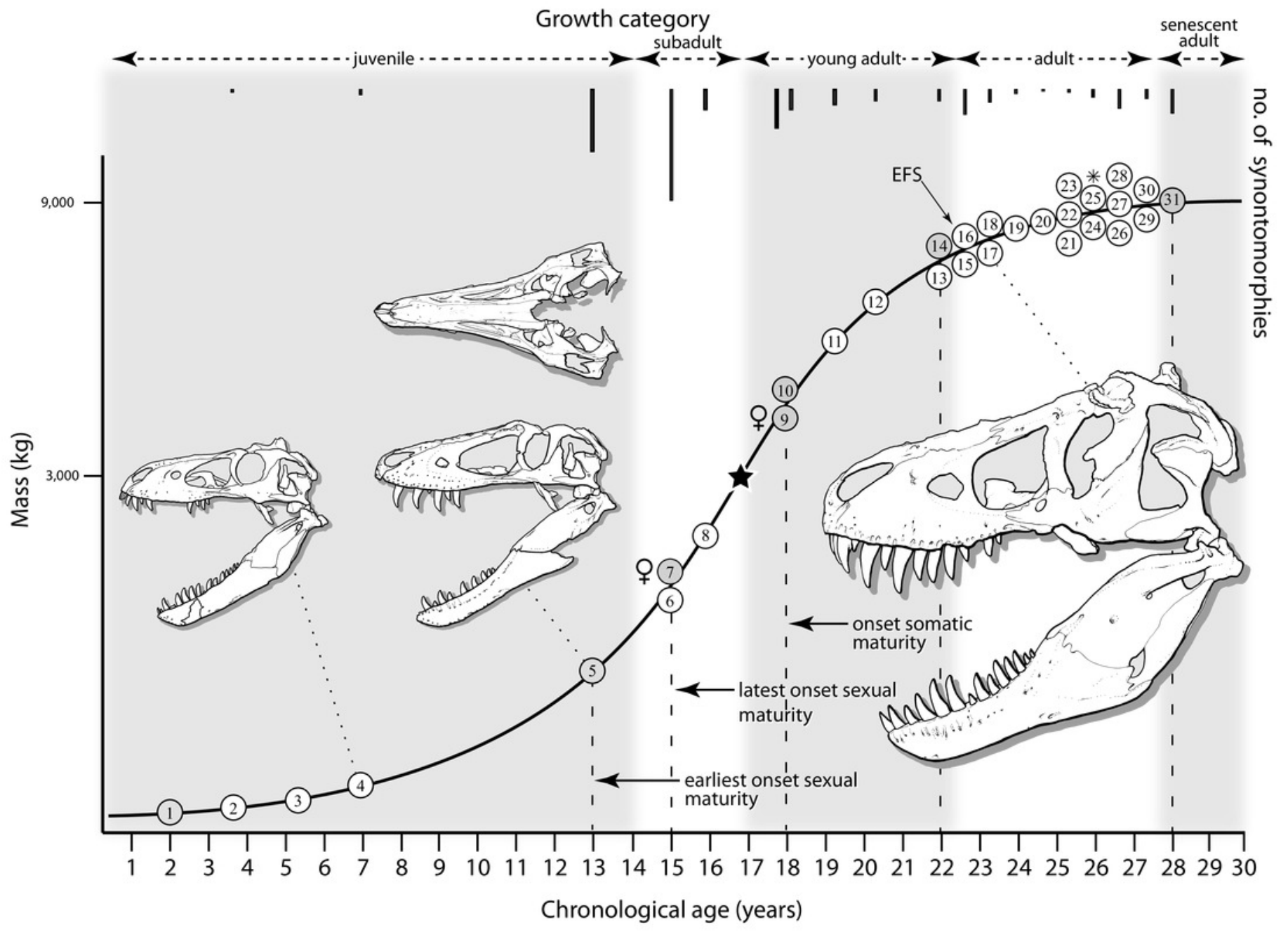




\section{Figure 13}

Scatterplot showing the congruence in Tyrannosaurus rex between bite force (i.e., and maturity.

Growth stage rank, corresponding to the increasing sequence of nodes in Figure 2, is along the x-axis; growth stage rank refers to the relative maturity of the specimens for which bite force has been estimated. Increasing bite force rank is along the $y$-axis; raw bite force data are from Bates and Falkingham (2012) and Gignac and Erickson (2017). A Spearman correlation test on these data resulted in a significant correlation coefficient, indicating that bite force increases with maturity.

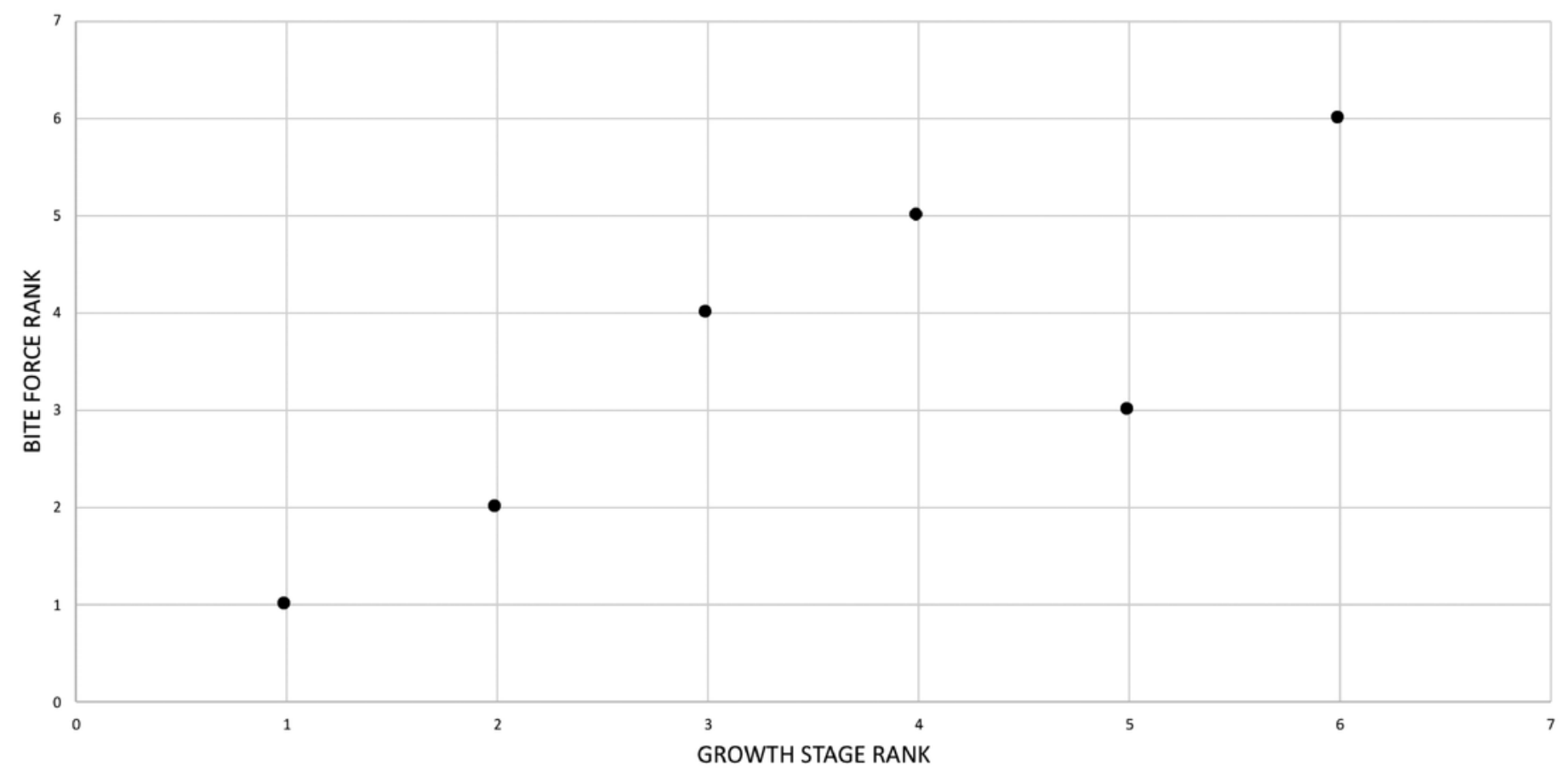




\section{Figure 14}

Bivariate scatterplot showing the relationship between maxillary tooth count with maturity among 14 specimens of Tyrannosaurus rex.

Growth rank increases away from the origin (i.e., maturity increases to the right) and corresponds to growth stages for which maxillary tooth count was available for a given specimen; i.e., the rank does not correspond to growth stage. Maxillary tooth rank corresponds to relative tooth count, where low ranks correspond to high tooth counts and low ranks correspond to high tooth counts.

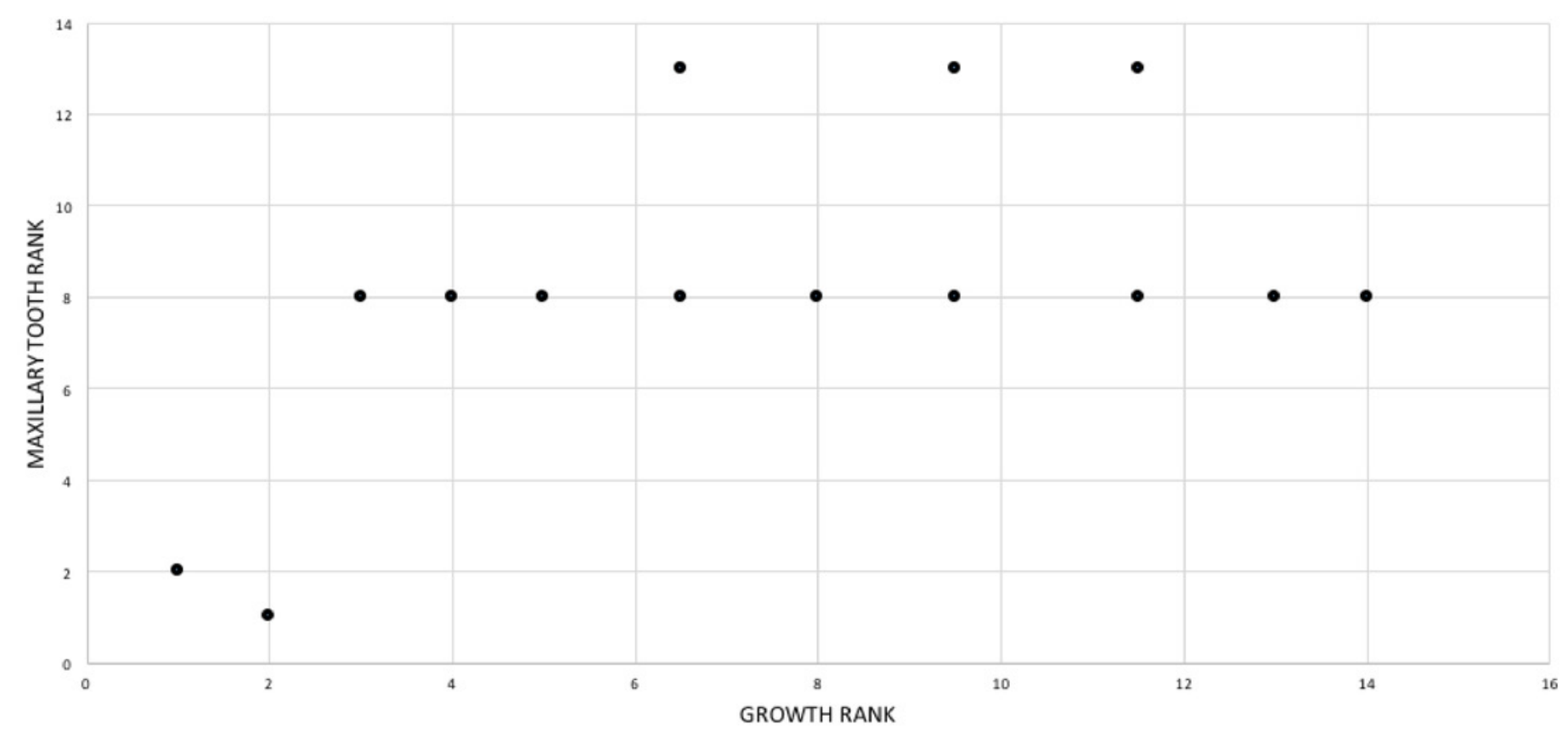




\section{Figure 15}

Bivariate scatterplot showing the relationship between dentary tooth count with maturity among 16 specimens of Tyrannosaurus rex.

Growth rank increases away from the origin (i.e., maturity increases to the right) and corresponds to growth stages for which dentary tooth count was available for a given specimen; i.e., the rank does not correspond to growth stage. Dentary tooth rank corresponds to relative tooth count, where low ranks correspond to high tooth counts and low ranks correspond to high tooth counts.

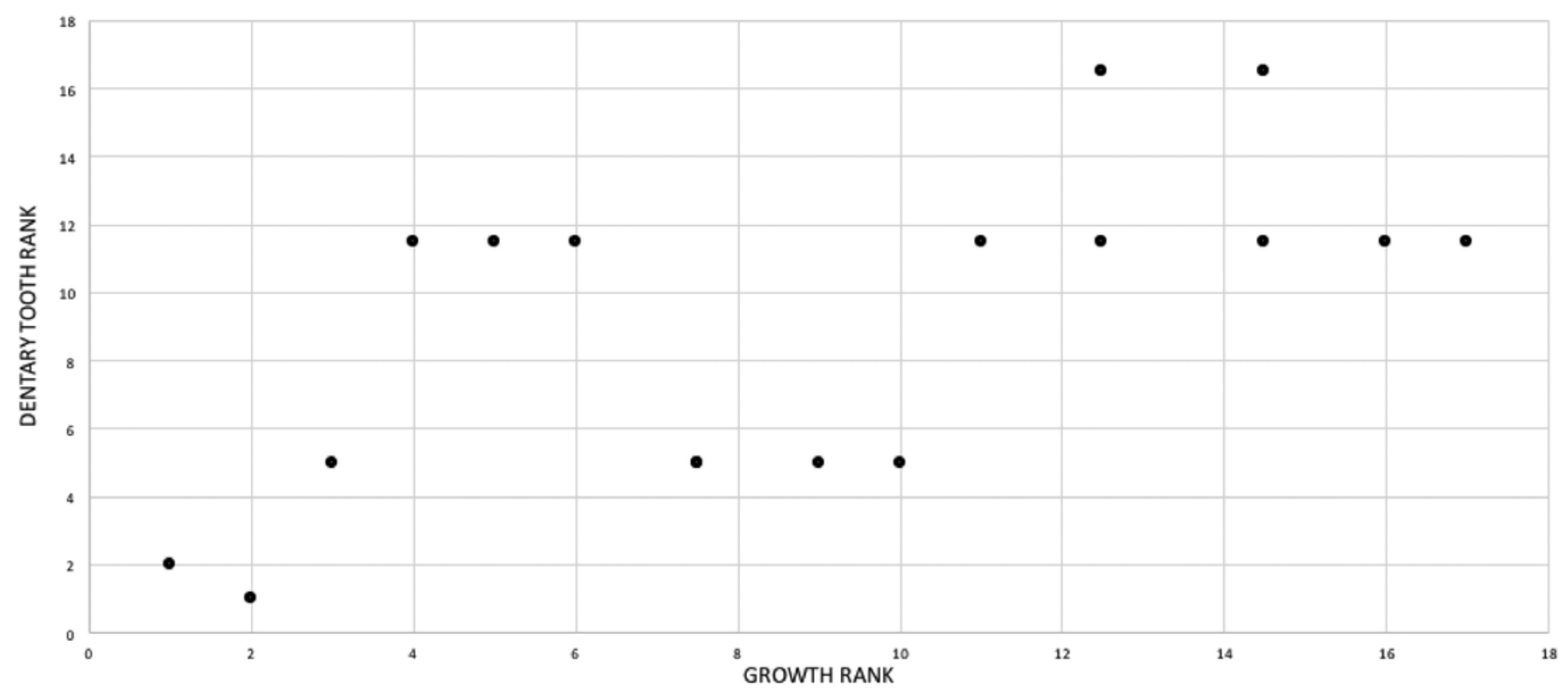


Figure 16

Bivariate scatterplot showing the relationship between chronological age with maturity among eight specimens of Tyrannosaurus rex.

The comparison is limited to specimens that have been histologically aged; growth stages ( $\mathrm{x}$ axis) and chronological age (y-axis) have been converted to ranks. See Table 14 for the raw data.

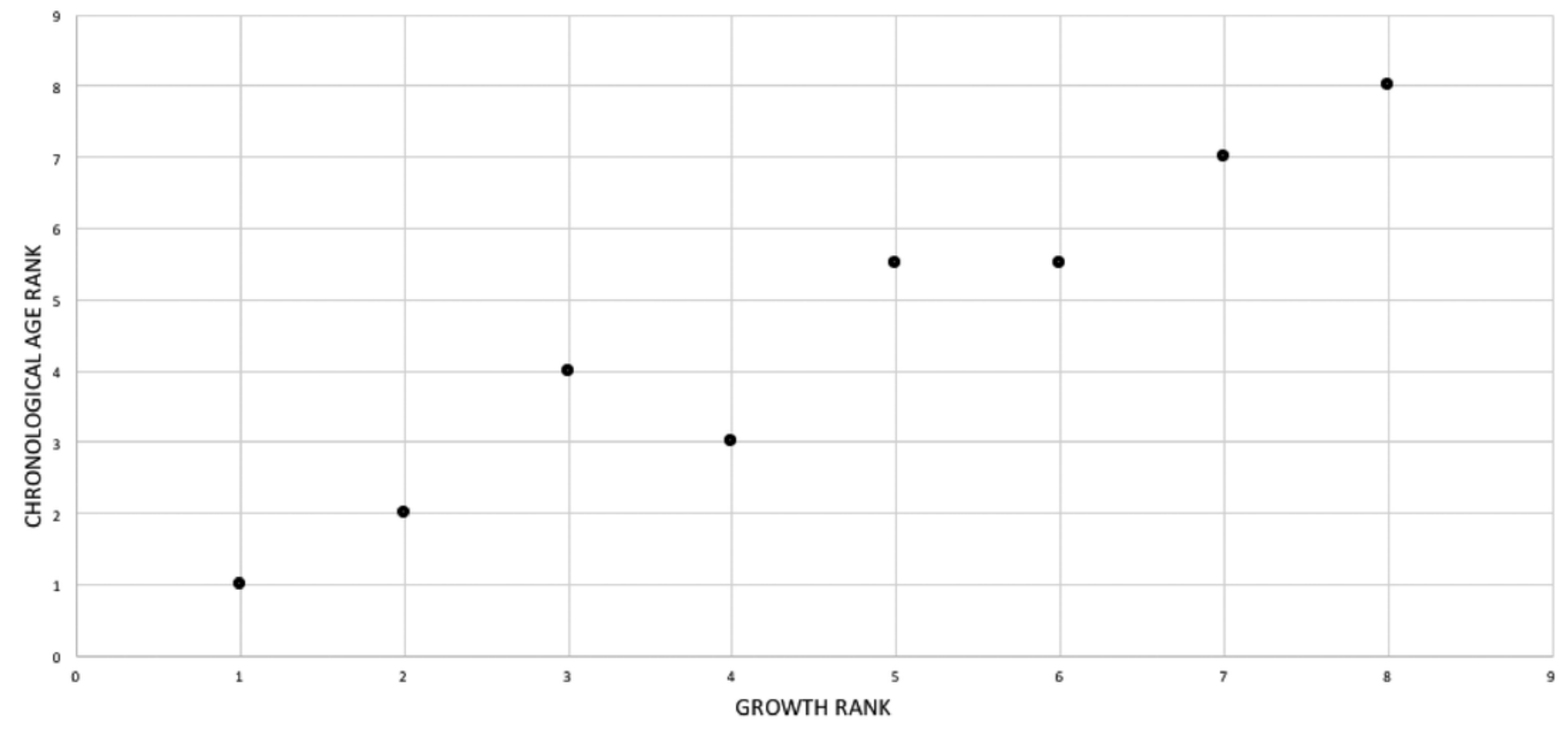


Figure 17

Bivariate scatterplots showing the relationship between size with maturity among 15 specimens of Tyrannosaurus rex.

The comparison is limited to specimens that have comparable size data; growth stages ( $\mathrm{x}$ axis) and size (y-axis) have been converted to ranks. See Table 15 for the raw data.

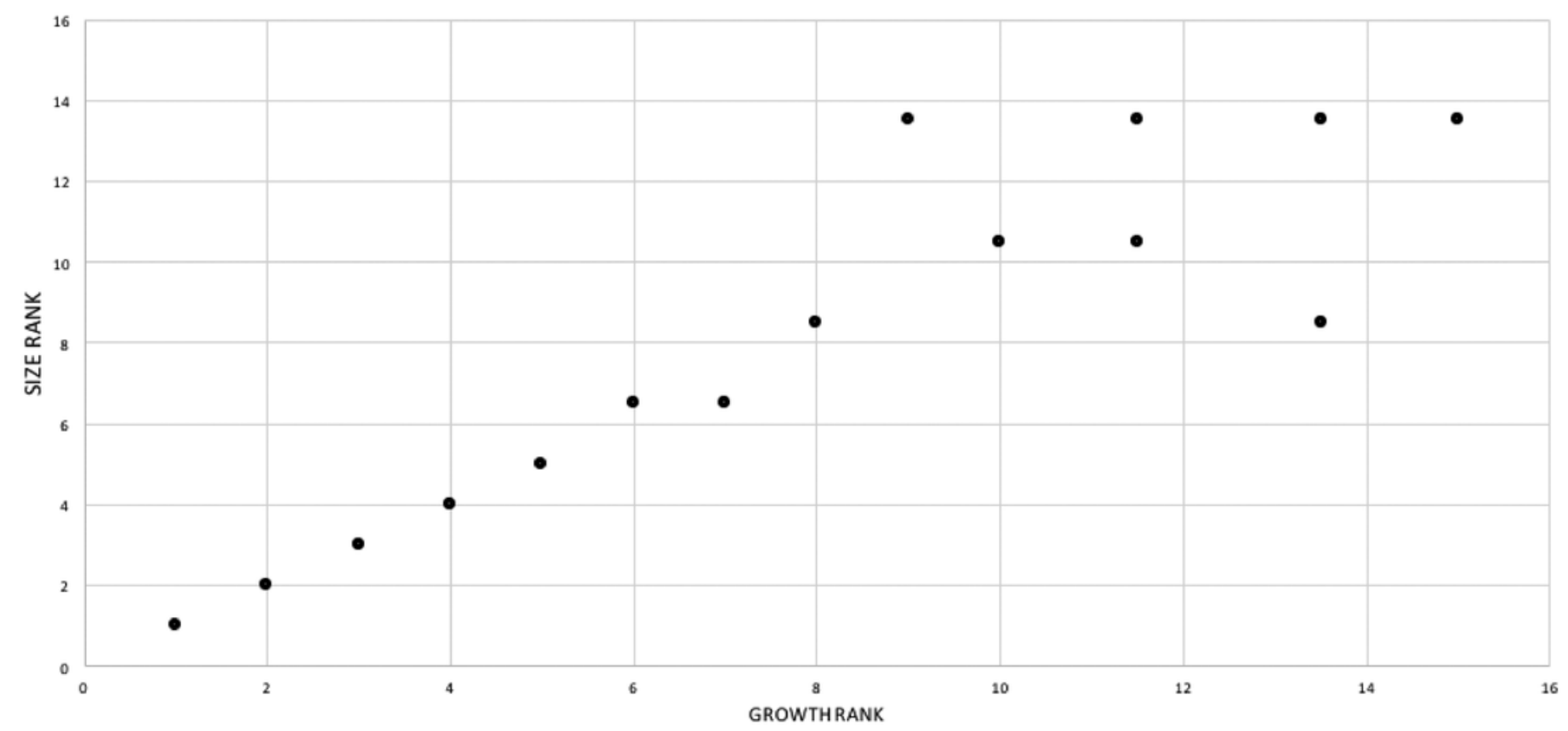




\section{Figure 18}

Bivariate scatterplots showing the relationship between mass with maturity among nine specimens of Tyrannosaurus rex.

The comparison is limited to specimens that have published mass estimates; growth stages (x-axis) and mass (y-axis) have been converted to ranks. See Table 16 for the raw data.

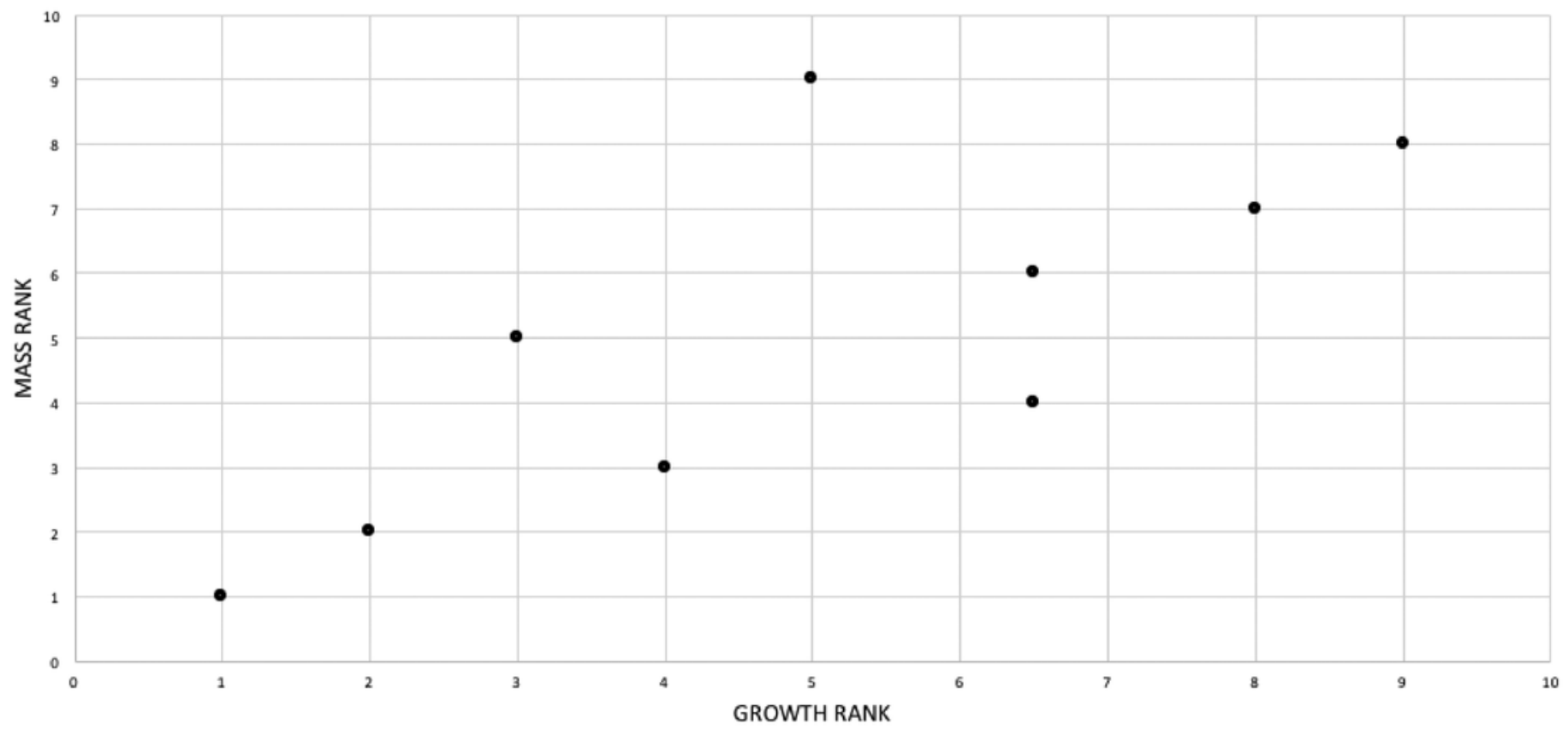




\section{Figure 19}

Bivariate scatterplot showing the relationship between geographic location with maturity among 28 specimens Tyrannosaurus rex.

Growth stages (x-axis) and geographic location (y-axis) have been converted to ranks. See Table 17 for the ranked data. "Montana North" refers to the region of Dawson, Garfield, and McCone counties, and "Montana South" refers to the region of Yellowstone and Carter counties. Maturity increases to the right along the x-axis; the $y$-axis follows the north-south axis of North America.

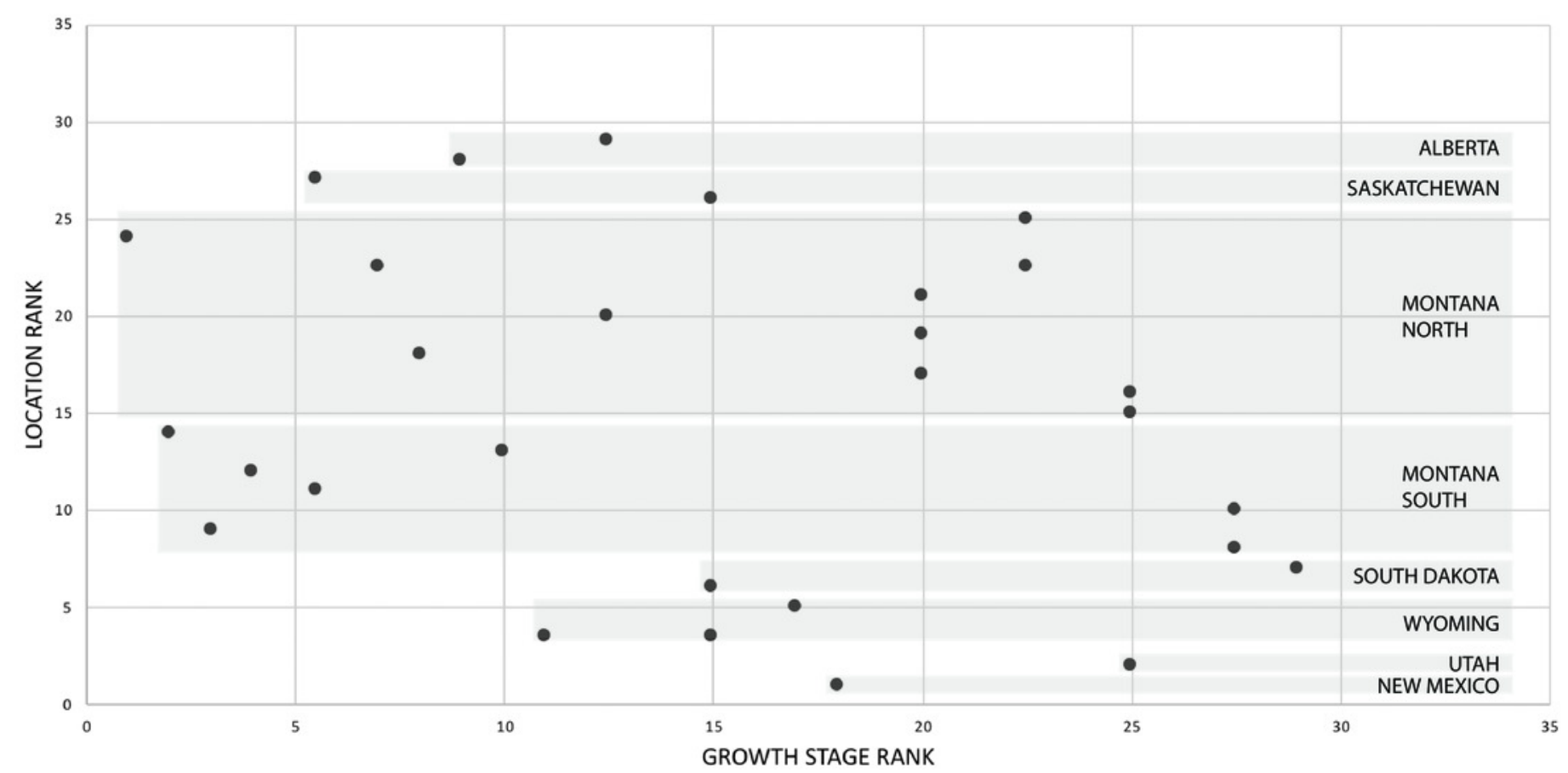




\section{Figure 20}

Bivariate scatterplot showing the relationship between stratigraphic position with maturity among nine specimens of Tyrannosaurus rex.

Growth stages (x-axis) and stratigraphic position (y-axis) have been converted to ranks. See Table 18 for the raw and ranked data. Maturity rank increases to the right; stratigraphic rank decreases from the origin (i.e., the upper HCF is closest to the origin, whereas the lower HCF is furthest from the origin).

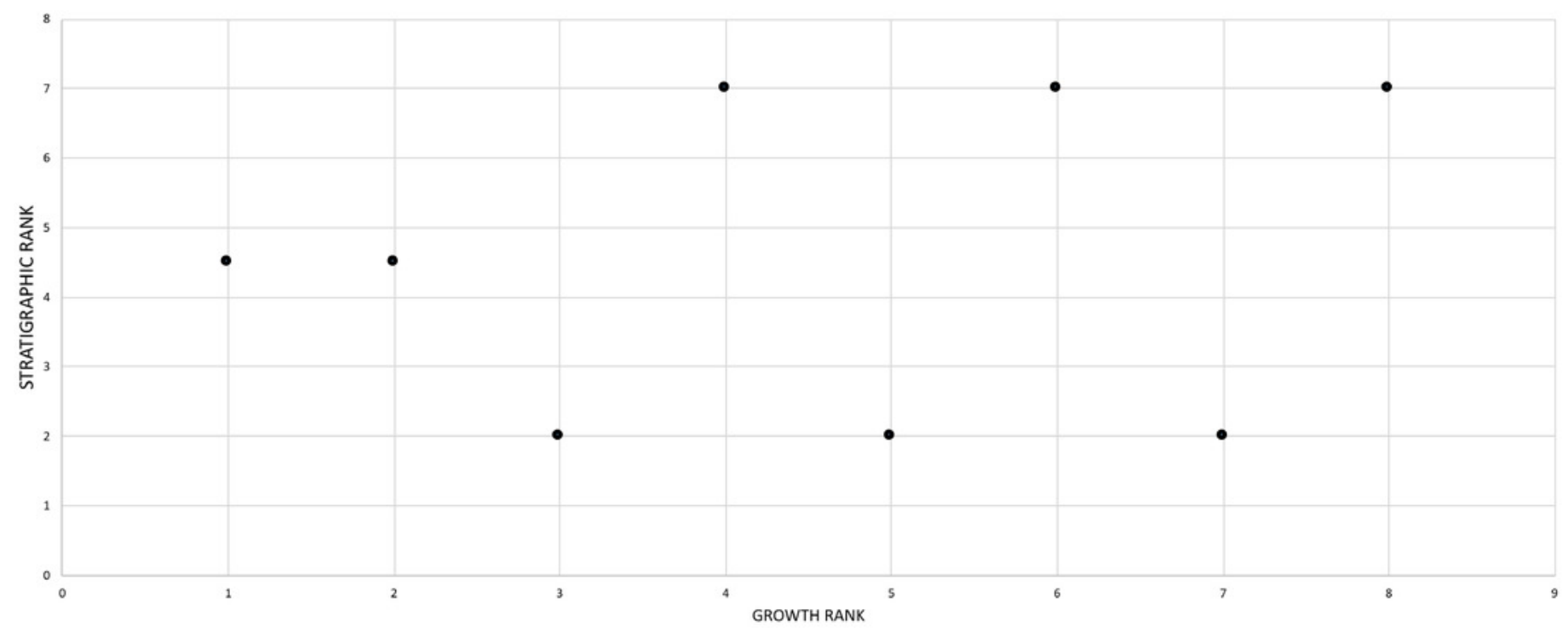




\section{Figure 21}

Sex dimorphs and the taxon "Tyrannosaurus " $x$ "" of Larson (2008) mapped onto the ontogram of Tyrannosaurus rex.

A transitional pattern is not seen between gracile and robust morphs; if sexual dimorphism was present, then the "gracile" and "robust" morphs should group along separate branches, which is not seen. Also, specimens referred to the taxon " $T$. " $x$ "” do not form a clade, indicating that it is not a valid taxon. The pattern seen here is what is expected for a species without sexual dimorphism. Specimens considered in Larson (2008) as gracile are in boldface italics with a boldface "G"; specimens considered in Larson (2008) as robust are in boldface with a boldface "R"; specimens considered in Larson (2008) as referable to " $T$. " $x$ " are in italics and marked with an " $X$ ". 


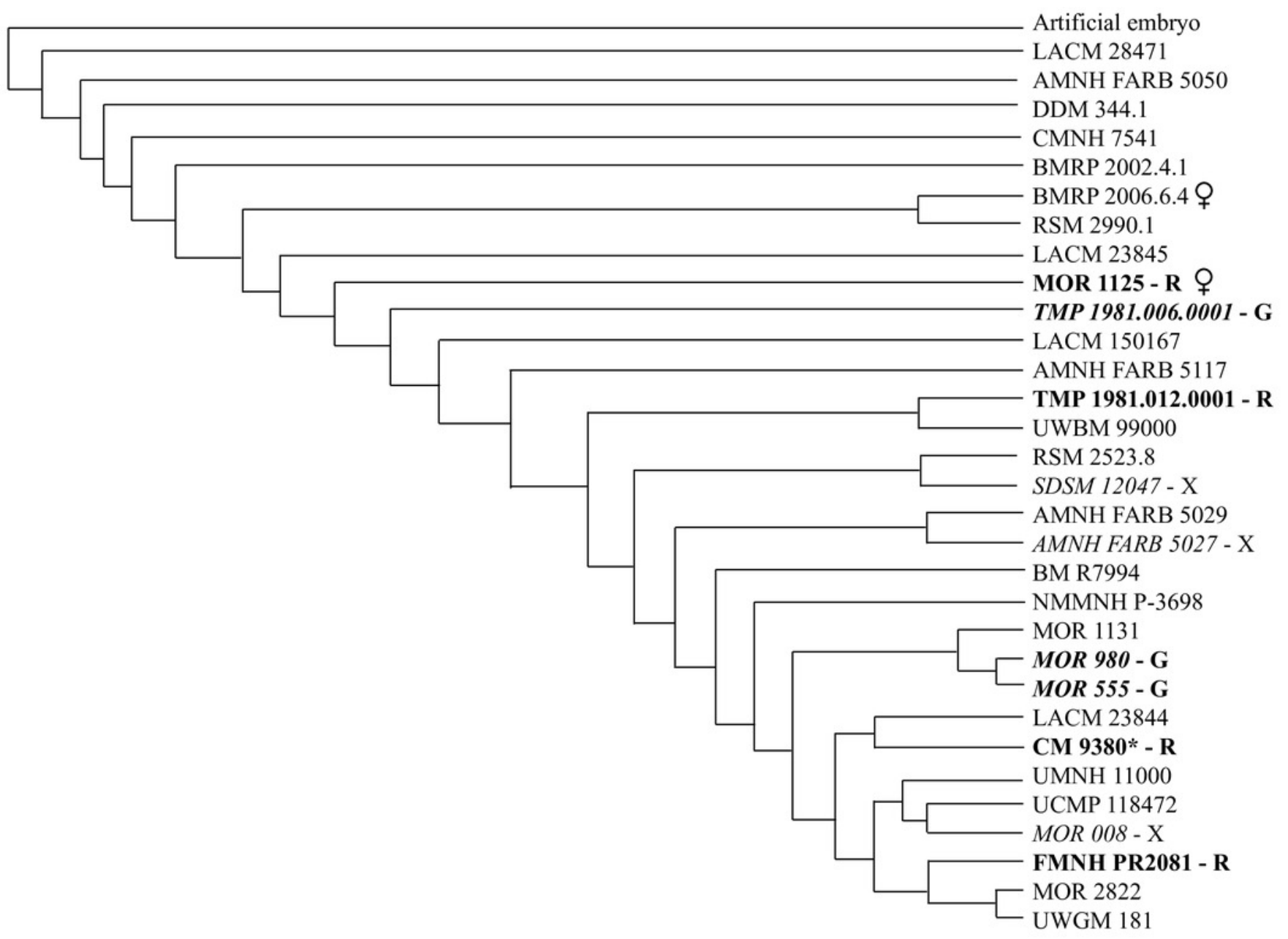




\section{Figure 22}

Sex dimorphs of Larson (2008) mapped onto the growth curve of Tyrannosaurus rex.

A transitional pattern is not seen between gracile and robust morphs; if sexual dimorphism was present, then the "gracile" and "robust" morphs should grade into each other, which is not seen. Likewise, an ontogenetic progression among the cranial and postcranial indices is not seen. See text for details. Key to specimens numbered on the growth curve is in Figure 12.

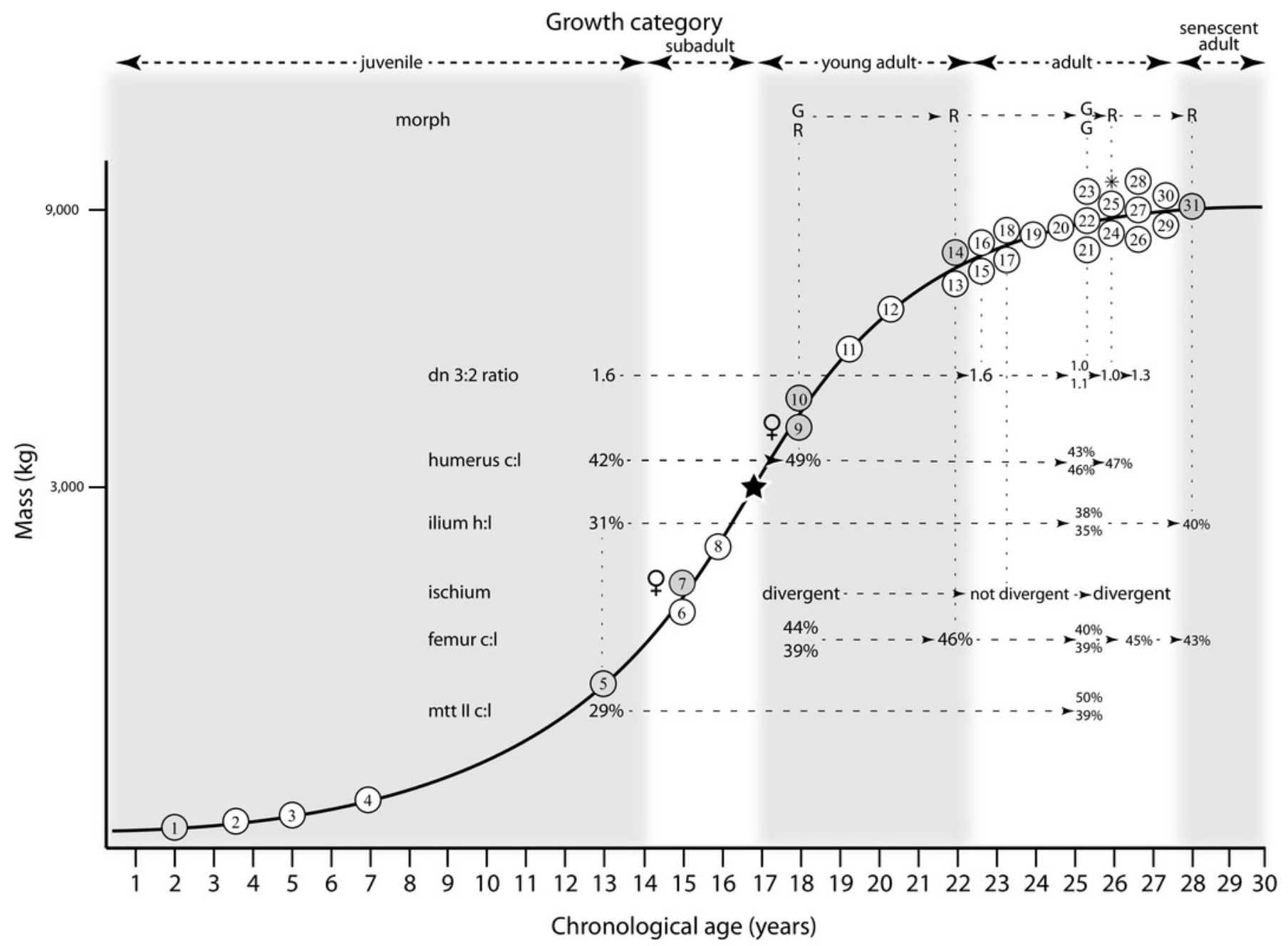




\section{Figure 23}

The results of Henderson (2002) mapped onto the growth curve of Tyrannosaurus rex.

All measures of the correlates of orbital fenestra size and shape change from juvenile to adult categories. It is predicted here that this transition occurred early in ontogeny, at the subadult growth stage, given the presence of correlates of a tall skull in subadult specimens. Key to specimens numbered on the growth curve is in Figure 12.

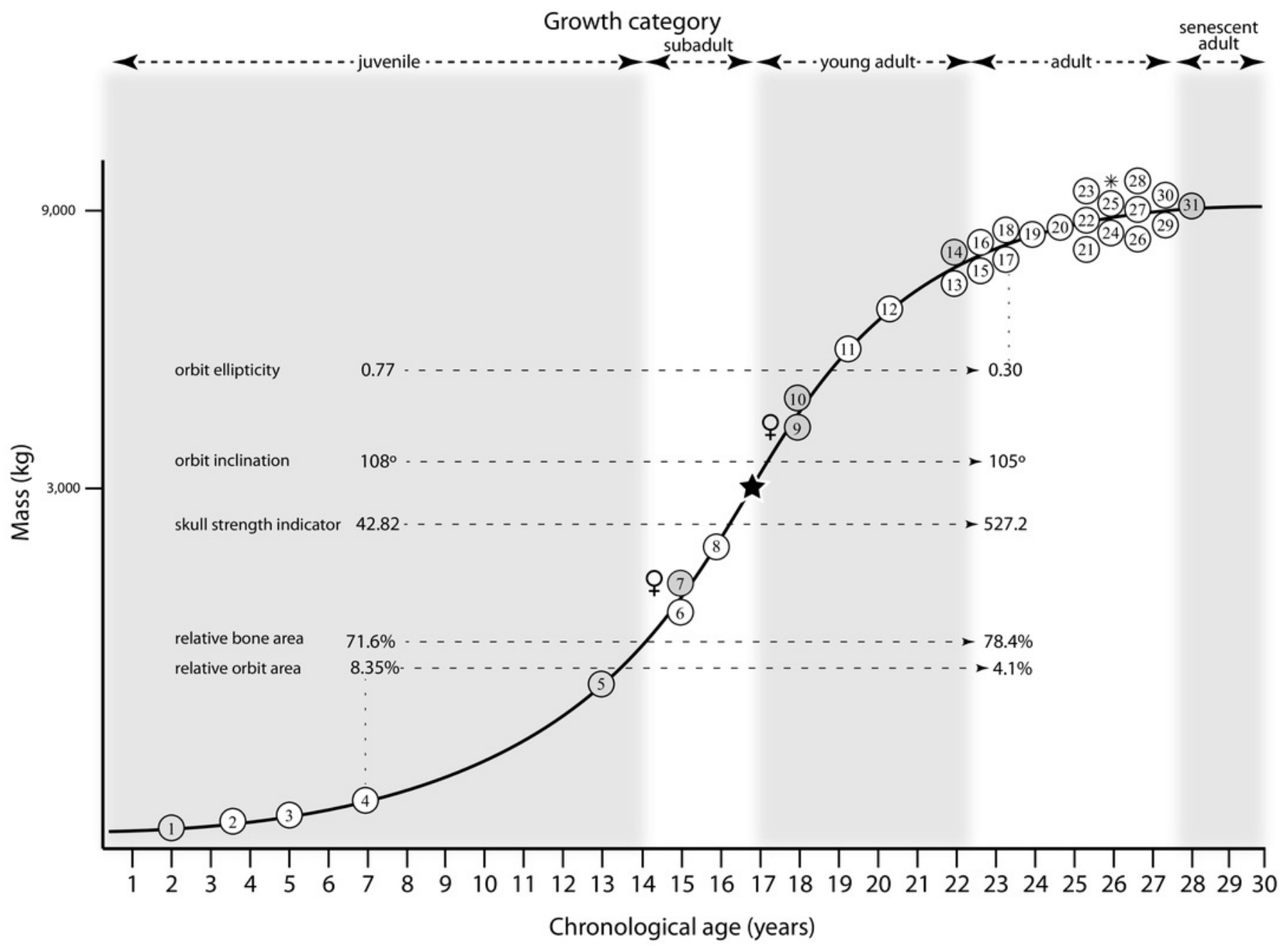




\section{Figure 24}

Skull bending strength mapped onto the growth curve of Tyrannosaurus rex.

The results of Snively et al. (2006) showing that the subadult growth stage was an important functional transition point during ontogeny between the long and low skulls of adults and tall and sturdy skulls of more mature animals. Their results show a progression in strength of the skull frame and dentition throughout the adult categories. Key to specimens numbered on the growth curve is in Figure 12.

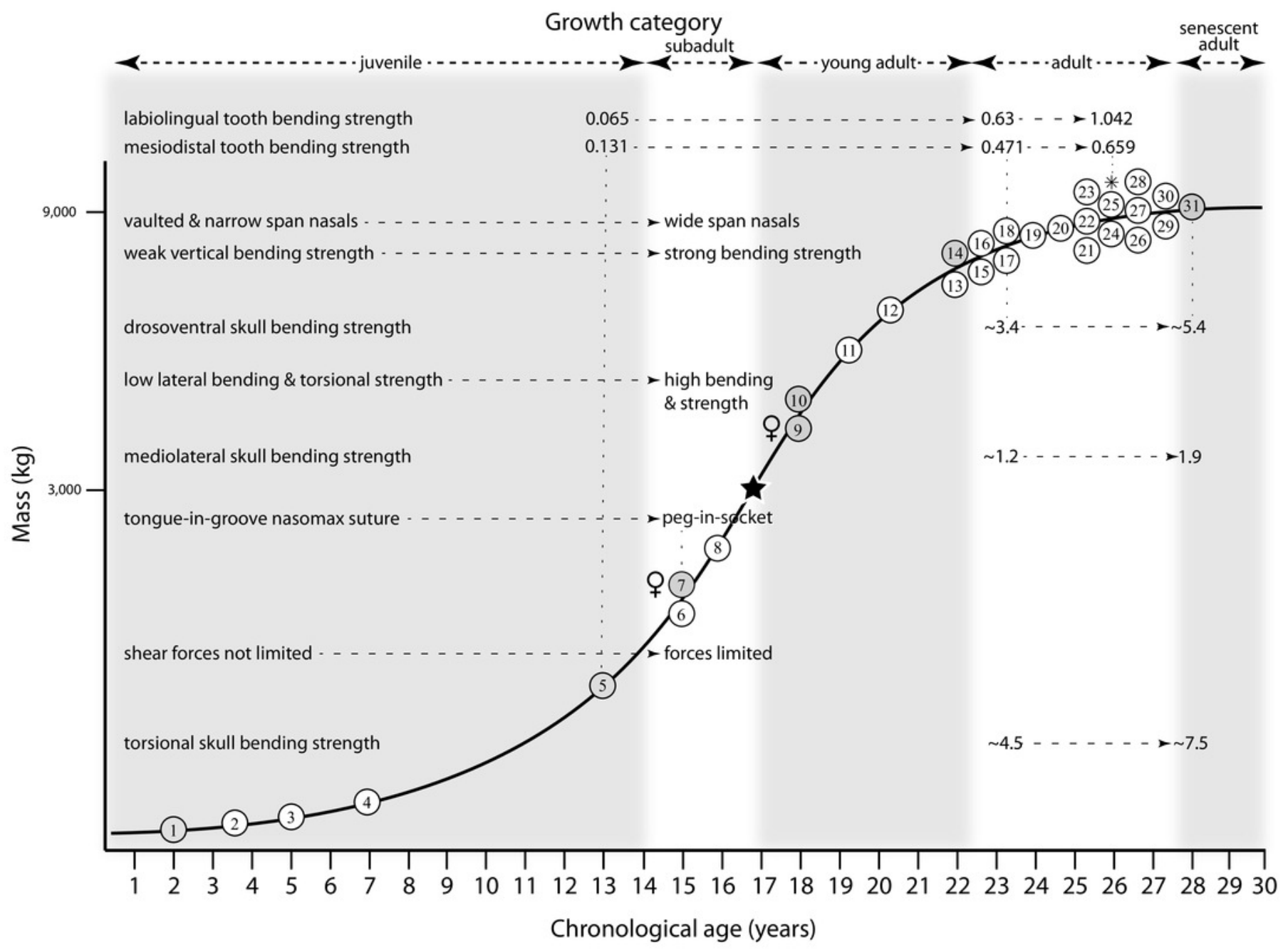




\section{Figure 25}

The results of Therrien et al. (2005) compared with the growth curve of Tyrannosaurus rex.

Vertical bending strength and relative bending strength (sensu Therrien et al., 2005) mapped onto the growth curve of $T$. rex. Values for juveniles are missing for mid-dentary dorsoventral strength and mid-dentary relative strength. In general, strength increases ontogenetically, a trend that becomes obscured in adulthood. Key to specimens numbered on the growth curve is in Figure 12.

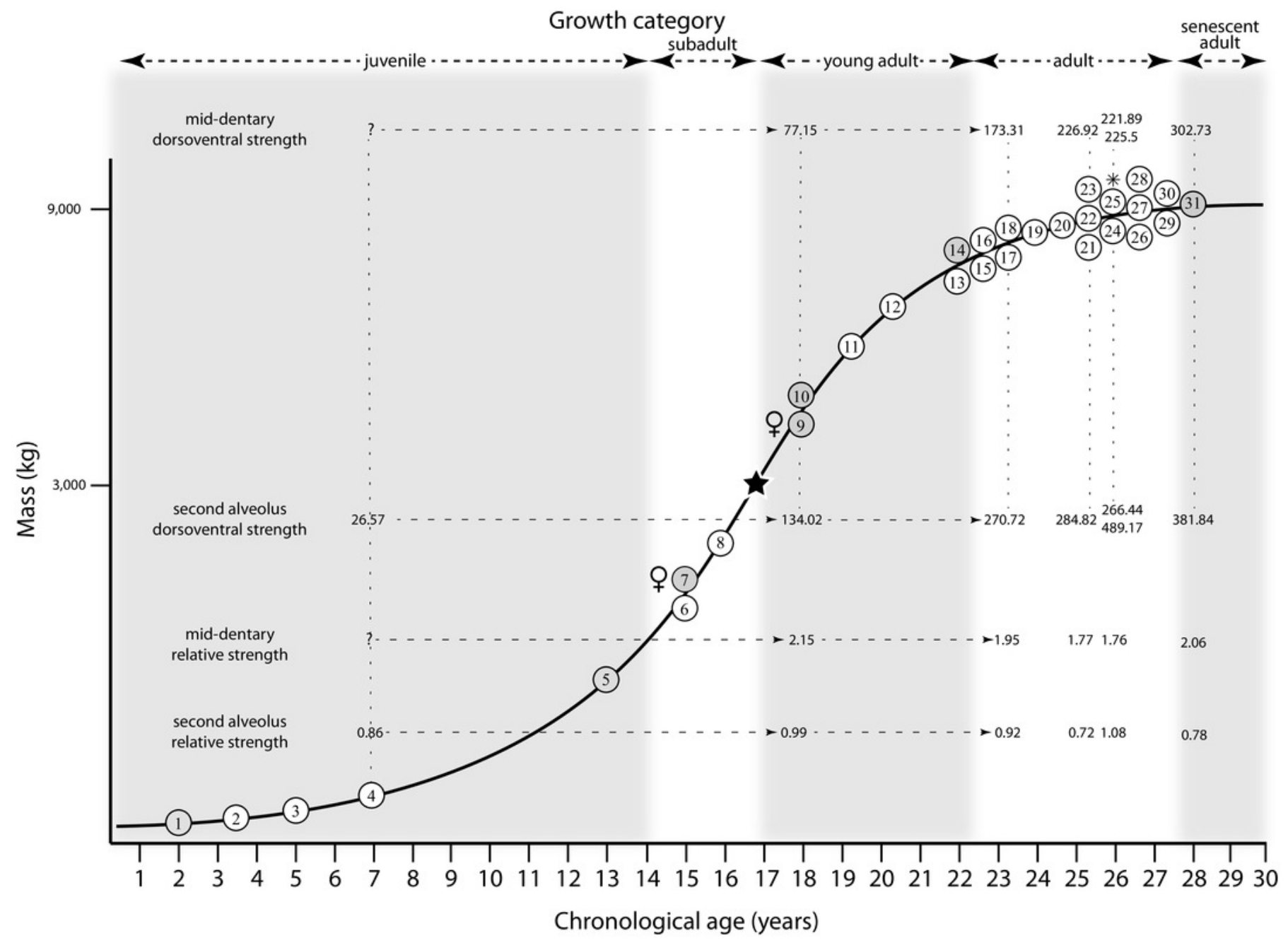




\section{Figure 26}

Heat maps of the ontogenetic changes seen in the skull and mandible of Tyrannosaurus rex.

Illustrations show per centage of the total number of unambiguously optimized synontomorphies per bone (A) and functional module (B). Darker shades of gray indicate higher proportions of growth change, whereas lighter shades indicate lower proportions of change. The results show that the greatest amount of growth changes are at the lacrimal (A) or along the dorsal skull roof (B). Hatchure indicates empty space; stipple indicates unprepared matrix.
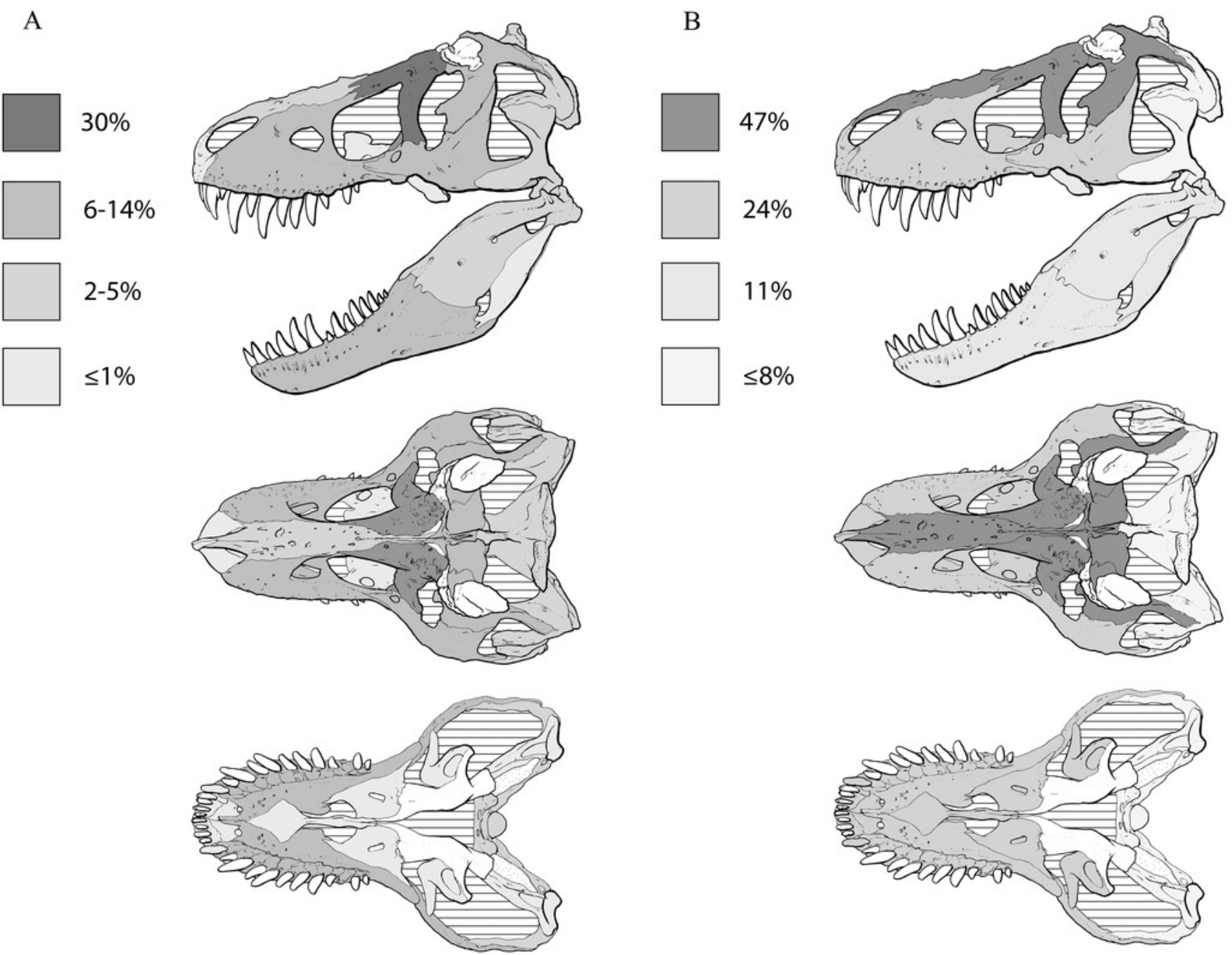


\section{Figure 27}

Comparison of the results of Henderson and Snively (2004) with the growth curve of Tyrannosaurus rex.

The rotational inertia (RI) of smaller and progressively distant sister taxa of $T$. rex serve as predictive proxies for the RIs of young adult and juvenile $T$. rex. Given the larger size of $T$. rex in contrast to non-tyrannosaurine tyrannosaurids, the RI of young adult $T$. rex will almost certainly be more comparable to that of adult $D$. torosus than to adult $A$. libratus. The positions of the taxa, aside from T. rex (FMNH PR2081), are relative and are not intended to correspond to exact locations along the growth curve. Key to specimens numbered on the growth curve is in Figure 12. 


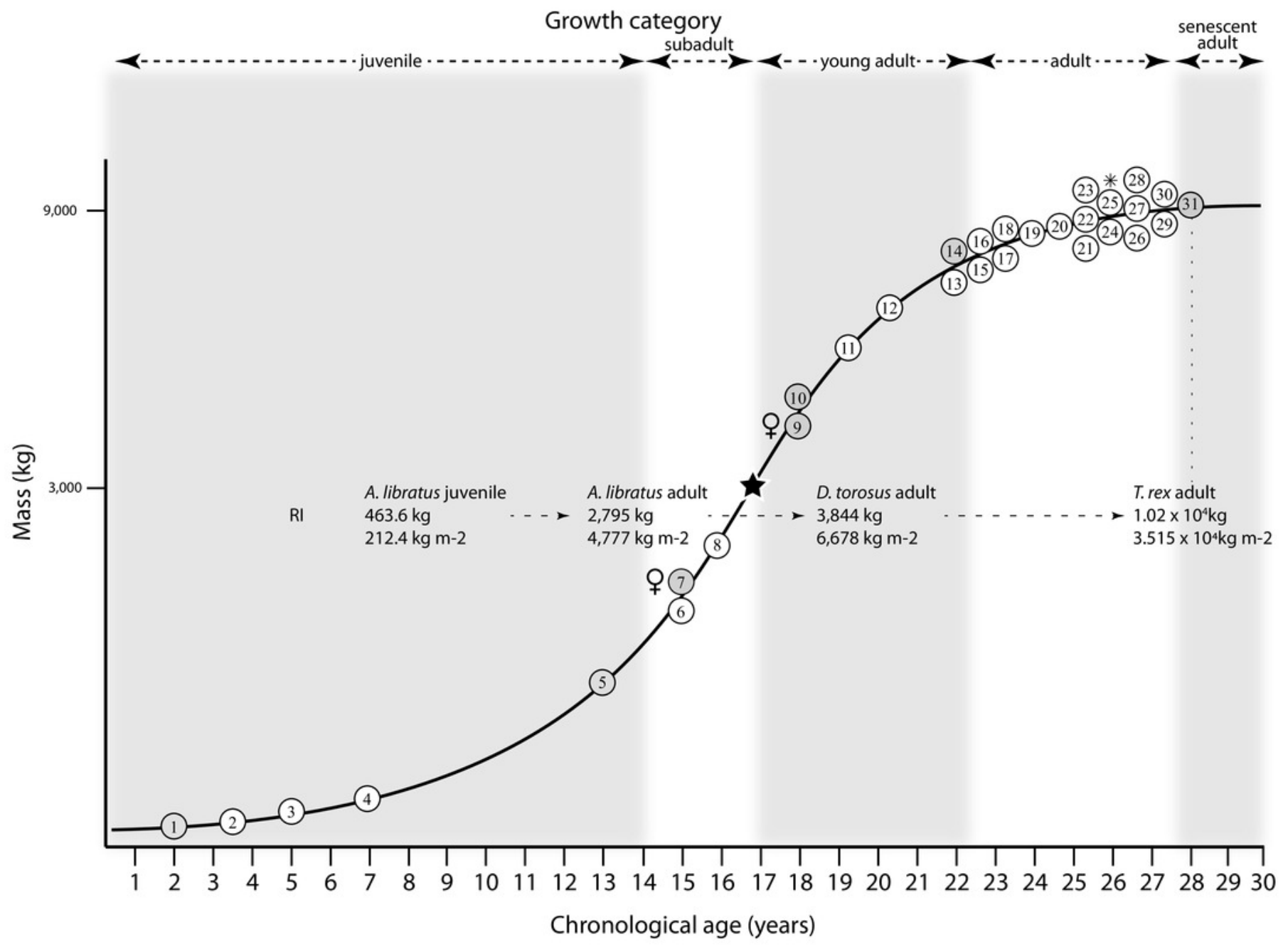




\section{Figure 28}

Comparison of the results of Snively et al. (2019) with the growth curve of Tyrannosaurus rex.

A comparison of the agility values between $T$. rex and other tyrannosaurids. The values for $T$. bataar and $D$. torosus serve as predictive proxies for the corresponding values in subadult and young adult $T$. rex. The values for $T$. rex are calibrated to the growth series, but those of the other taxa are positioned relative to the values seen in $T$. rex. Inset of the data in table form shows the trends in the data; low values for Albertosaurus libratus are in boldface italics. Key to specimens numbered on the growth curve is in Figure 12. Abbreviations: Al, Albertosaurus libratus; Dt, Daspletosaurus torosus; Thb, Tyrannosaurus bataar; $\mathbf{T r}$, Tyrannosaurus rex. 


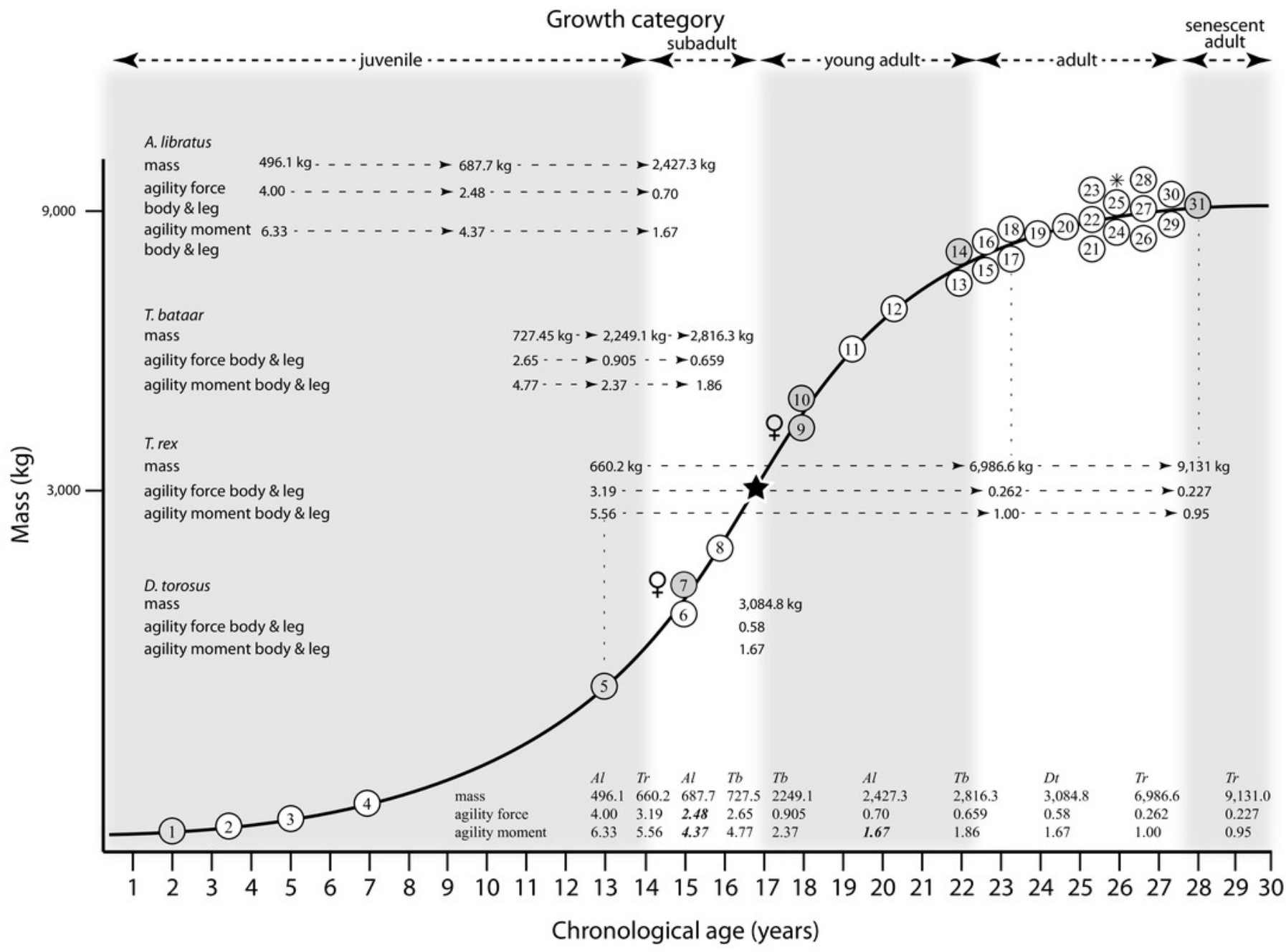




\section{Figure 29}

Reptile Encephalization Quotients (REQs) of Hurlburt et al. (2013) mapped onto the growth curve of Tyrannosaurus rex.

The REQ is based on a brain mass to endocranial volume ratio of $37 \%$ and the parenthetical values following the REQs corresponds to the two di $\square$ erent body mass estimates, in metric tonnes, from which the REQs were derived (see Hurlburt et al., 2013 for details). Overall, the REQ of the juvenile greatly exceeds that of adults, and the adults show an increasing ontogenetic progression of REQ values, as $\square$ rst reported by Hurlburt et al. (2013). Key to specimens numbered on the growth curve is in Figure 12.

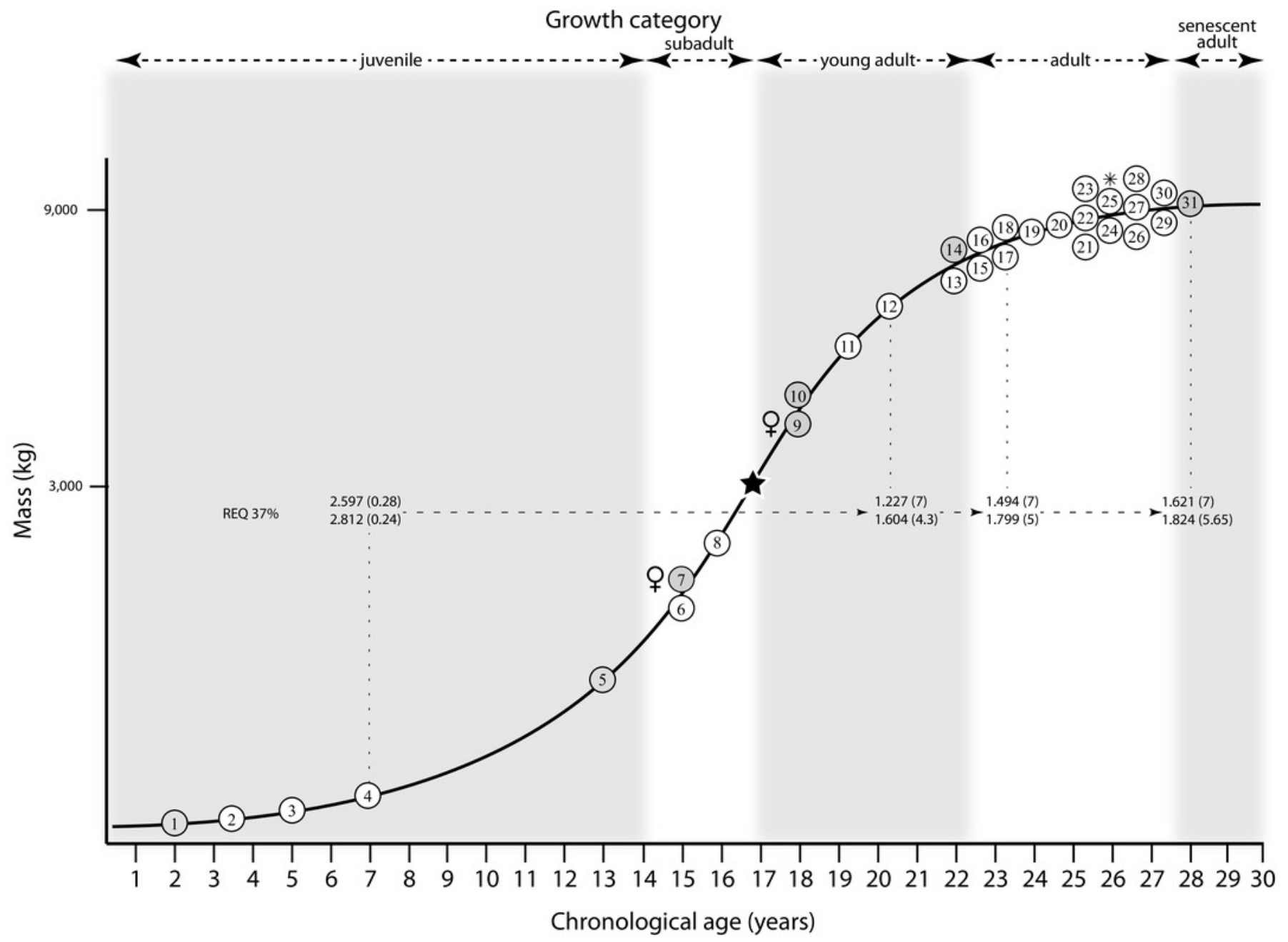




\section{Figure 30}

Comparison of recapitulatory synontomorphies of Tyrannosaurus rex with tyrannosauroid phylogeny.

Ten unambiguously optimized synontomorphies are congruent with unambiguously optimized synapomorphies of tyrannosauroid phylogeny, providing limited evidence of recapitulation (see text for details). Numbers to the right correspond to the growth stages in Figure 2. If recapitulation was present, then the growth stage numbers should increase with progressively exclusive clades; that pattern is not seen here. 


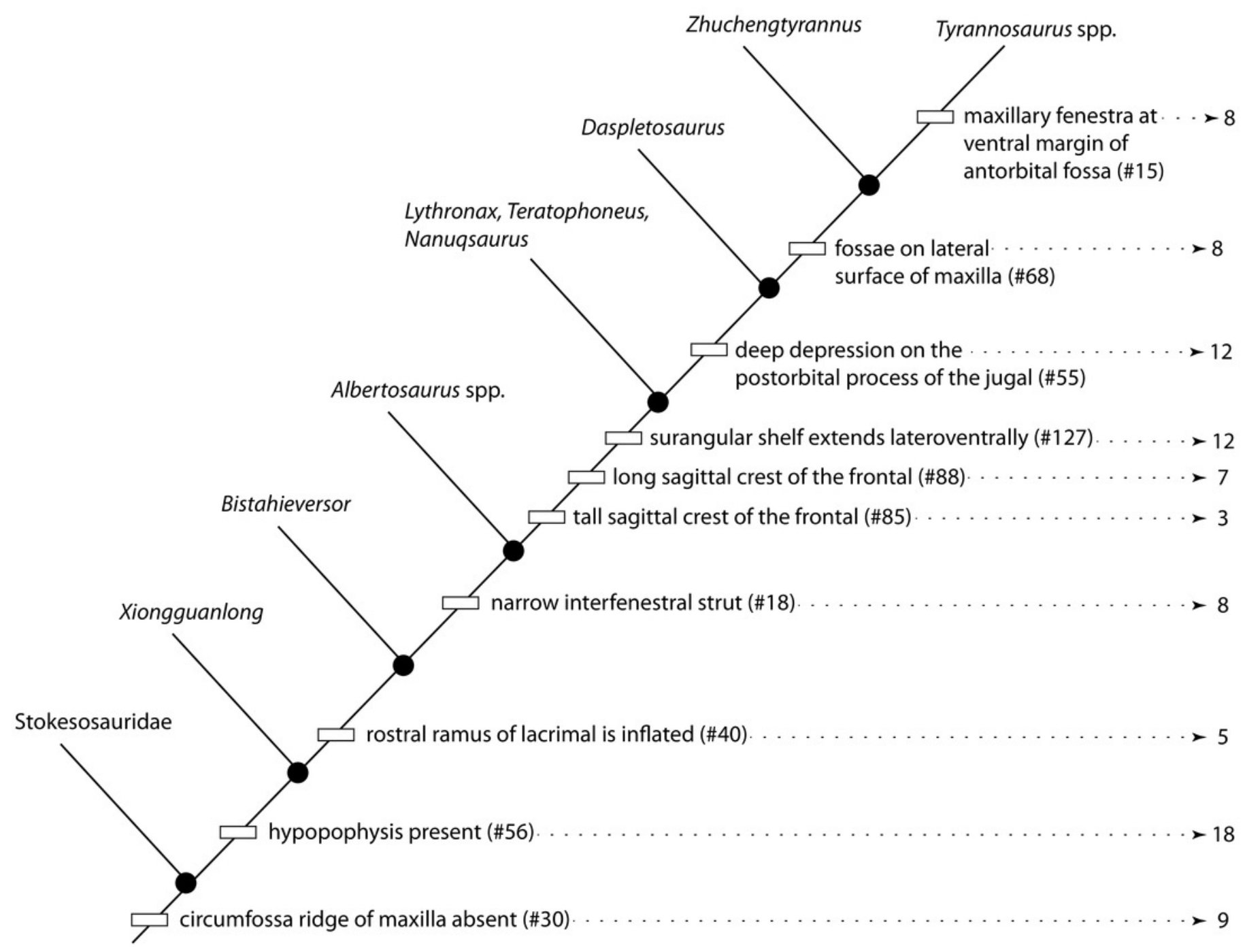




\section{Figure 31}

Bivariate scatterplot showing the test of ontogenetic recapitulation of phylogenetic novelties in Tyrannosaurus rex.

Growth stage rank (increases away from the origin) is along the x-axis; clade rank (increases away from the origin) is along the y-axis. If recapitulation is present, then the ranks will increase montonically from the origin. A recapitulatory pattern is not seen in $T$. rex; see text for details.

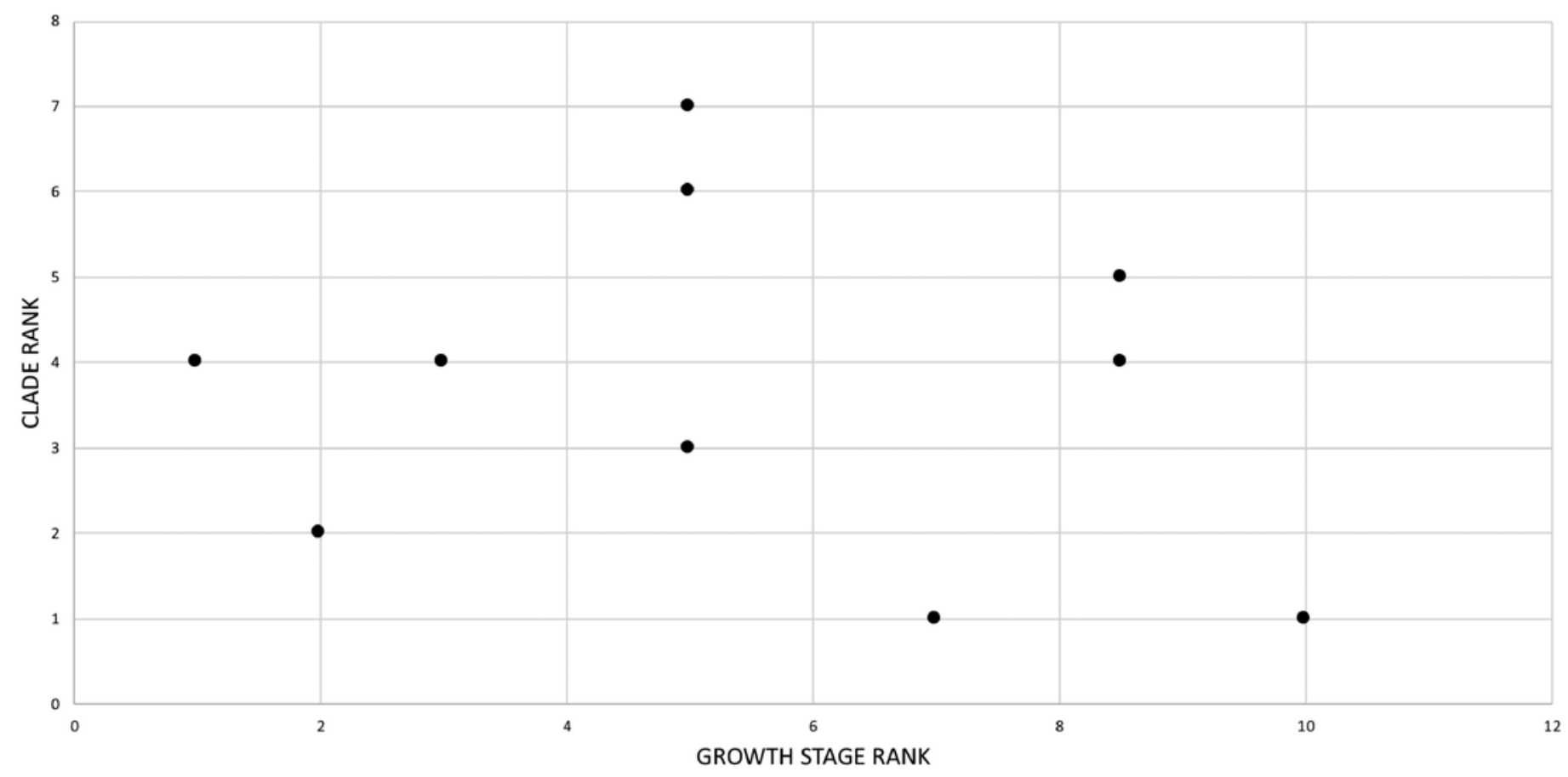




\section{Figure 32}

Bivariate scatterplot showing the congruence between individual variation per specimen per node compared with the number of unambiguously optimized synontomorphies per node in Tyrannosaurus rex.

The number of synontomorphies per node are along the x-axis; the amount of individual variation (i.e., unambiguously optimized character states per branch) is along the y-axis. Both values increase away from the origin. If the amount of individual variation is controlled by the number of synontomorphies per node, then the variables should increase monotonically. In this case, no congruence is seen between the variables in T. rex.

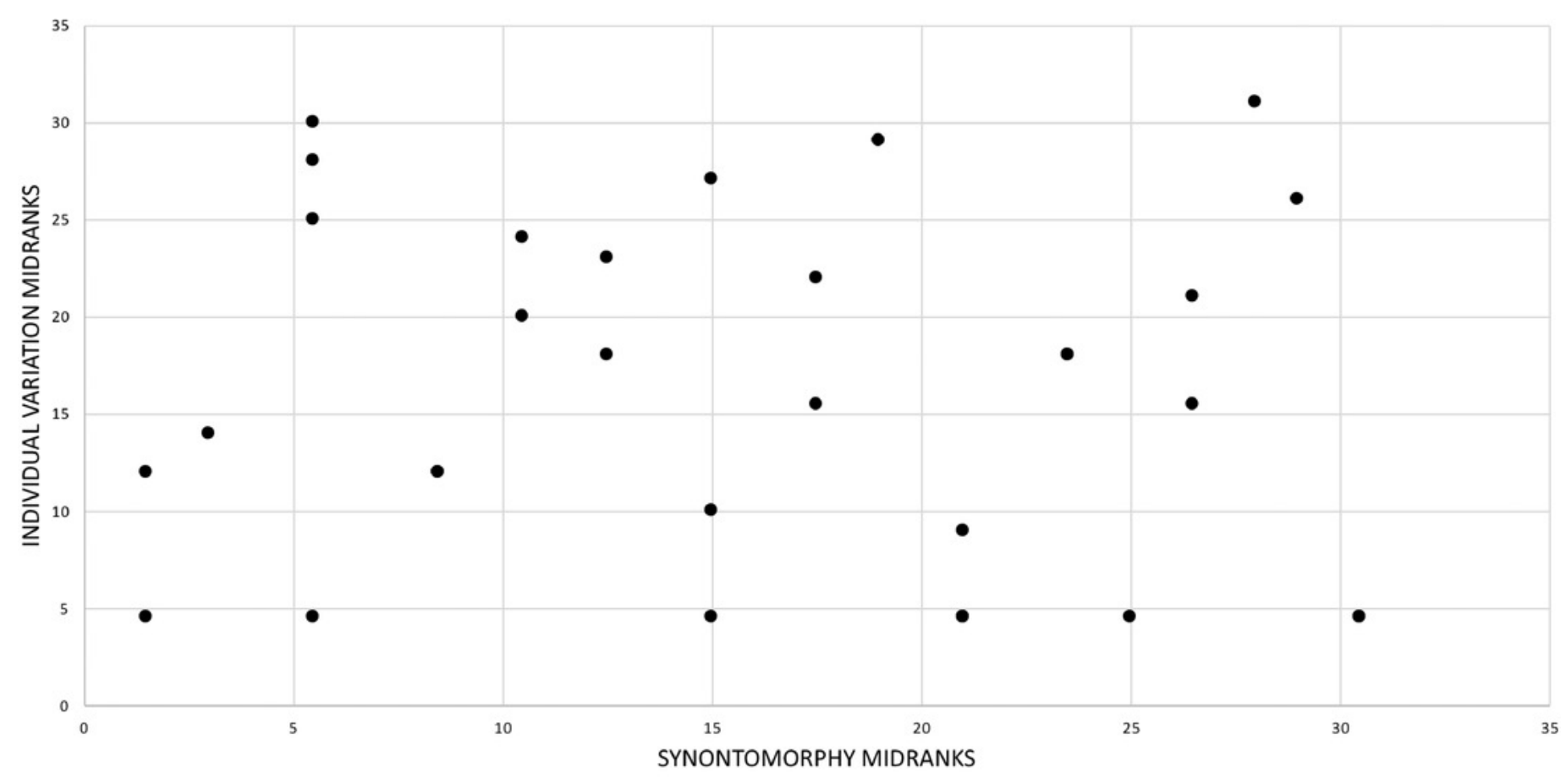




\section{Figure 33}

A simplified cladogram of living and extinct Archosauriformes showing 13 cranial and postcranial growth changes that are optimized as synapomorphies.

Most of the growth changes are ancestral for Archosauriformes. The position of several characters at progressively exclusive clades is almost certainly an artifact of missing data (e.g., increase in mandible height, enlargement of muscle attachments, etc.) and they are predicted to be synapomorphic for Archosauriformes once the appropriate data are acquired. This comparison shows that highly derived species such as Tyrannosaurus rex do not deviate from the ancestral growth trends that first evolved in significantly smaller taxa. See text for sources; see Table 23 for the distribution of character states among the taxa. 


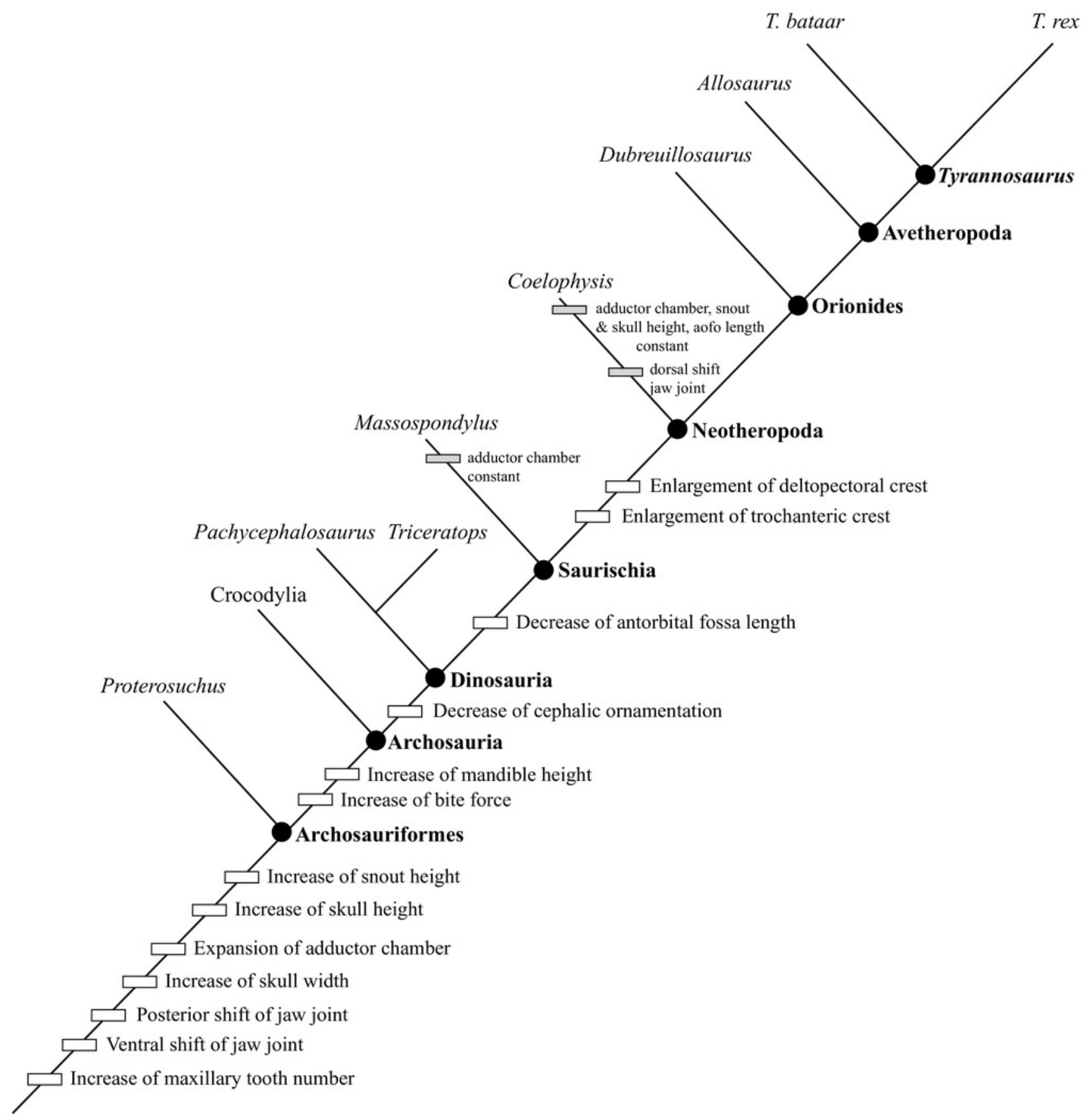




\section{Table $\mathbf{1}$ (on next page)}

Summary of completeness of the Tyrannosaurus rex specimens included in this analysis with identi $\square$ cation of the wildcard specimens.

Summary of the completeness of the specimens of $T$. rex included in this study, given as the percentage of missing characters and the percentage of characters present out of a total of 1,850 . The list is given in descending order, where the least complete specimen is at the top of the table and the most complete is at the bottom. Grey [II indicates the 13 wildcard specimens. 
1

\begin{tabular}{|c|c|c|}
\hline Specimen & $\%$ missing characters & $\%$ present characters \\
\hline BMRP 2001.4.70 & 99.8 & 0.2 \\
\hline DDM 1863.1 & 99.7 & 0.3 \\
\hline DDM 1562.14 & 99.6 & 0.4 \\
\hline MOR 002 & 99.4 & 0.6 \\
\hline DDM 1536.8 & 98.9 & 1.1 \\
\hline DDM 35.1 & 98.6 & 1.4 \\
\hline TMP 1981.012.0001 & 98.2 & 1.8 \\
\hline AMNH FARB 5050 & 98.2 & 1.8 \\
\hline CM 9401 & 98.1 & 1.9 \\
\hline UMNH 11000 & 97.8 & 2.2 \\
\hline UCMP 118742 & 97.6 & 2.4 \\
\hline RSM 2347.1 & 97.3 & 2.7 \\
\hline TMM 41436-1 & 97.1 & 2.9 \\
\hline MOR 2925 & 96.5 & 3.5 \\
\hline DDM 344.1 & 96.3 & 3.7 \\
\hline FMNH PR2411 & 94.6 & 5.4 \\
\hline RSM 2990.1 & 94.6 & 5.4 \\
\hline MOR 3044 & 94.4 & 5.6 \\
\hline NMMNH P-3698 & 94.2 & $\begin{array}{l}5.8 \\
\end{array}$ \\
\hline CM 1400 & 93.9 & 6.1 \\
\hline AMNH FARB 5029 & 93.4 & 6.6 \\
\hline BMRP 2006.6.4 & 93.2 & 6.8 \\
\hline MOR 1131 & 92.9 & 7.1 \\
\hline BM R7994 & 92.6 & 7.4 \\
\hline UWGM 181 & 91.5 & 8.5 \\
\hline LACM 28471 & 89.6 & 10.4 \\
\hline AMNH FARB 5117 & 88.2 & 11.8 \\
\hline SDSM 12047 & 87.7 & 12.3 \\
\hline MOR 2822 & 83.3 & 16.7 \\
\hline LACM 23845 & 83.2 & 16.8 \\
\hline TMP 1981.006.0001 & 82.4 & 17.6 \\
\hline CM 9380 & 75.7 & 24.3 \\
\hline CMNH 7541 & 71.8 & 28.2 \\
\hline LACM 23844 & 62.4 & 37.6 \\
\hline AMNH FARB 5027 & 60.8 & 39.2 \\
\hline RSM 2523.8 & 59.5 & 40.5 \\
\hline MOR 008 & 57.6 & 42.4 \\
\hline MOR 555 & 52.7 & 47.3 \\
\hline UWBM 99000 & 49.3 & 50.7 \\
\hline LACM 150167 & 44.1 & 55.9 \\
\hline MOR 980 & 42.4 & 57.6 \\
\hline MOR 1125 & 42.2 & 57.8 \\
\hline FMNH PR2081 & 15.7 & 84.3 \\
\hline
\end{tabular}


BMRP 2002.4.1 13.3

86.7

2

3

4

5

6

7

8 


\section{Table 2 (on next page)}

An assessment of evidence for sexual dimorphism in Tyrannosaurus rex based on character states that are unambiguously optimized as individual variation.

The female specimen MOR 1125 shares homologous character transformations with $\square$ ve other specimens, but these shared changes are not common to all of the specimens, which would be expected if they represented diagnostic character states unique to one sex. Based on this lack of pattern it is inferred that character states unique to females are absent and, by extension, the skeleton of $T$. rex is not sexually dimorphic. 
1

\begin{tabular}{|c|c|c|c|c|c|}
\hline MOR 1125 & CMNH 7541 & $\begin{array}{l}\text { LACM } \\
150167 \\
\end{array}$ & MOR 555 & $\begin{array}{l}\text { BMRP } \\
\text { 2002.4.1 }\end{array}$ & $\begin{array}{l}\text { AMNH } \\
\text { FARB 5117 }\end{array}$ \\
\hline 330. Maxilla, subnrl frmn, dpth, $0=>1$ & & $330,0=>1$ & & & \\
\hline 419. Maxilla, js for jgl, rstrl extnt, $0=>1$ & & & $419,0=>1$ & & \\
\hline $\begin{array}{l}\text { 449. Lacrimal, drsl rms, sbctns srfc, } \\
\text { hght, } 0=>1\end{array}$ & & & & $449,0=>1$ & \\
\hline $\begin{array}{l}\text { 618. Postorbital, drsl mrgn, ornnttn, } \\
1=>2\end{array}$ & & $618,0=>1$ & & & \\
\hline $\begin{array}{l}\text { 728. Quadratojugal, sq pr, rstrl srfc, } \\
1=>2\end{array}$ & & & $728,1=>2$ & & \\
\hline $\begin{array}{l}\text { 963. Parietal, sggtl crst, cdl end, extnt, } \\
0==>1\end{array}$ & $963,0==>1$ & & & & \\
\hline 973. Parietal, prtsoc pllr, prsnc, $1=>2$ & & & & & $973,1=>2$ \\
\hline $\begin{array}{l}\text { 1022. Basioccipital, occ con, cdl mrgn, } \\
0=>1\end{array}$ & & & & & $1022,0=>1$ \\
\hline
\end{tabular}

2

3

4

5 


\section{Table 3(on next page)}

Comparison of the completeness of Tyrannosaurus rex specimens included in the backbone ontogram and the number of synontomorphies at each node.

Raw data are in normal typeface, ranks used in the correlation test are in boldface. 
1

\begin{tabular}{|c|c|c|c|c|c|c|}
\hline Specimen & $\begin{array}{l}\text { Specimen } \\
\text { complete } \\
\text { ness }\end{array}$ & $\begin{array}{l}\text { Specimen } \\
\text { completene } \\
\text { ss rank }\end{array}$ & $\begin{array}{l}\text { Specimen } \\
\text { completeness } \\
\text { midranks }\end{array}$ & $\begin{array}{l}\# \\
\text { synontomorphie } \\
\mathrm{s} \text { at node }\end{array}$ & $\begin{array}{l}\text { \#synontom } \\
\text { orphies } \\
\text { rank }\end{array}$ & $\begin{array}{l}\# \\
\text { synontomo } \\
\text { rphies } \\
\text { midranks }\end{array}$ \\
\hline BMRP 2002.4.1 & 86.7 & 1 & 1 & 51 & 3 & 3 \\
\hline FMNH PR2081 & 84.3 & 2 & 2 & 7 & 18 & 19 \\
\hline MOR 1125 & 57.8 & 3 & 3 & 31 & 4 & 4 \\
\hline MOR 980 & 57.6 & 4 & 4 & 2 & 25 & 26.5 \\
\hline LACM 150167 & 55.9 & 5 & 5 & 12 & 12 & 12 \\
\hline UWBM 99000 & 50.7 & 6 & 6 & 9 & 15 & 16 \\
\hline MOR 555 & 47.3 & 7 & 7 & 2 & 26 & 26.5 \\
\hline MOR 008 & 42.4 & 8 & 8 & 15 & 8 & 9.5 \\
\hline RSM 2523.8 & 40.5 & 9 & 9 & 20 & 5 & 5.5 \\
\hline AMNH FARB 5027 & 39.2 & 10 & 10 & 10 & 13 & 13.5 \\
\hline LACM 23844 & 37.6 & 11 & 11 & 6 & 21 & 21.5 \\
\hline CMNH 7541 & 28.2 & 12 & 12 & 3 & 23 & 23.5 \\
\hline CM 9380 & 24.3 & 13 & 13 & 6 & 22 & 21.5 \\
\hline TMP 1981.006.0001 & 17.6 & 14 & 14 & 16 & 7 & 7 \\
\hline LACM 23845 & 16.8 & 15 & 15 & 15 & 9 & 9.5 \\
\hline MOR 2822 & 16.7 & 16 & 16 & 7 & 19 & 19 \\
\hline SDSM 12047 & 12.3 & 17 & 17 & 20 & 6 & 5.5 \\
\hline AMNH FARB 5117 & 11.8 & 18 & 18 & 9 & 16 & 16 \\
\hline LACM 28471 & 10.4 & 19 & 19 & 0 & 30 & 30.5 \\
\hline UWGM 181 & 8.5 & 20 & 20 & 7 & 20 & 19 \\
\hline BM R7994 & 7.4 & 21 & 21 & 3 & 24 & 23.5 \\
\hline MOR 1131 & 7.1 & 22 & 22 & 2 & 27 & 26.5 \\
\hline BMRP 2006.6.4 & 6.8 & 23 & 23 & 89 & 1 & 1.5 \\
\hline AMNH FARB 5029 & 6.6 & 24 & 24 & 10 & 14 & 13.5 \\
\hline NMMNH P-3698 & 5.8 & 25 & 25 & 1 & 29 & 29 \\
\hline RSM 2990.1 & 5.4 & 26 & 26 & 89 & 2 & 1.5 \\
\hline DDM 344.1 & 3.7 & 27 & 27 & 0 & 31 & 30.5 \\
\hline UCMP 118742 & 2.4 & 28 & 28 & 15 & 10 & 9.5 \\
\hline UMNH 11000 & 2.2 & 29 & 29 & 15 & 11 & 9.5 \\
\hline AMNH FARB 5050 & 1.8 & 30 & 30.5 & 2 & 28 & 26.5 \\
\hline TMP 1981.012.0001 & 1.8 & 31 & 30.5 & 9 & 17 & 16 \\
\hline
\end{tabular}




\section{Table 4 (on next page)}

Summary of synontomorphies and individual variation in Tyrannosaurus rex organized by growth category and functional modules of the skull and jaws.

Summary of the number of unambiguously optimized changes recovered for the craniomandibular skeleton of Tyrannosaurus rex, organized by functional modules (sensu Werneburg et al., 2019). Individual variation is shown in parentheses. 
1

\begin{tabular}{llllllll}
\hline $\begin{array}{l}\text { Growth } \\
\text { category }\end{array}$ & Skull roof & $\begin{array}{l}\text { Snout }+ \\
\text { palate }\end{array}$ & Parietal & Suspensorium & Braincase & Mandible & Total \\
\hline $\begin{array}{l}\text { Small } \\
\text { juvenile }\end{array}$ & $3(3)$ & $0(1)$ & $0(2)$ & $0(0)$ & $0(0)$ & $2(0)$ & $5(6)$ \\
\hline $\begin{array}{l}\text { Large } \\
\text { juvenile }\end{array}$ & $15(6)$ & $30(5)$ & $0(0)$ & $1(0)$ & $0(0)$ & $7(3)$ & $53(14)$ \\
\hline Subadult & $58(2)$ & $0(0)$ & $0(2)$ & $0(0)$ & $0(0)$ & $0(1)$ & $58(5)$ \\
\hline Young adult & $17(38)$ & $13(23)$ & $11(10)$ & $5(8)$ & $14(8)$ & $7(11)$ & $67(98)$ \\
\hline Adult & $14(51)$ & $15(28)$ & $1(9)$ & $7(15)$ & $8(21)$ & $11(27)$ & $56(151)$ \\
\hline $\begin{array}{l}\text { Senescent } \\
\text { adult }\end{array}$ & $10(\mathrm{n} / \mathrm{a})$ & $2(\mathrm{n} / \mathrm{a})$ & $0(\mathrm{n} / \mathrm{a})$ & $2(\mathrm{n} / \mathrm{a})$ & $1(\mathrm{n} / \mathrm{a})$ & $0(\mathrm{n} / \mathrm{a})$ & $15(\mathrm{n} / \mathrm{a})$ \\
\hline Total & $117(100)$ & $60(57)$ & $12(23)$ & $13(23)$ & $25(29)$ & $27(42)$ & $254(274)$ \\
\hline
\end{tabular}

2

3 


\section{Table 5 (on next page)}

Summary of mass estimates for Tyrannosaurus rex.

Summary of published mass estimates $(\mathrm{kg})$ for individual specimens of Tyrannosaurus rex with rank order in each column in parentheses; sum rank order given in right-hand column. The rank of AMNH FARB 5027 could not be identified given its presence in only one analysis and the great difference in estimates between the analyses. Column numbers: 1, Erickson et al. (2004); 2, Henderson and Snively (2004); 3, Bates et al. (2009); 4, Hutchinson et al. (2011); 5, Campione et al. (2014); 6, Snively et al. (2019); 7, Persons et al. (2019) 
1

\begin{tabular}{|c|c|c|c|c|c|c|c|c|}
\hline Specimen & 1 & 2 & 3 & 4 & 5 & 6 & 7 & Rank \\
\hline LACM 28471 & $29.9(1)$ & & & & & & & 1 \\
\hline $\begin{array}{l}\text { BMRP } \\
2002.4 .1\end{array}$ & & & & $954(1)$ & & $660.2(1)$ & & 2 \\
\hline LACM 23845 & $1,807(2)$ & & & & & & & 3 \\
\hline $\begin{array}{l}\text { TMP } \\
1981.006 .0001\end{array}$ & $3,230(3)$ & & & & & & $4,469(1)$ & 4 \\
\hline $\begin{array}{l}\text { TMP } \\
1981.012 .0001\end{array}$ & $5,040(4)$ & & & & & & & 5 \\
\hline MOR 980 & & & & & & & $5,112(2)$ & 6 \\
\hline MOR 1125 & & & & & & & $6,100(3)$ & 7 \\
\hline $\begin{array}{l}\text { AMNH FARB } \\
5027\end{array}$ & & & & & & $6,986.6(2)$ & & $?$ \\
\hline MOR 555 & & & $6,071.8(1)$ & $8,272(2)$ & $6,216(1)$ & & $6,264(4)$ & 8 \\
\hline CM 9380 & & & & $9,081(3)$ & $6,688(2)$ & & $6,740(5)$ & 9 \\
\hline FMNH PR2081 & $5,654(5)$ & $\begin{array}{l}10,200 \\
\text { (1) }\end{array}$ & & $13,996(4)$ & $7,377(3)$ & $9,130.9(3)$ & $8,462(6)$ & 10 \\
\hline RSM 2523.8 & & & & & $8,004(4)$ & & $8,870(7)$ & 11 \\
\hline
\end{tabular}

2 


\section{Table 6(on next page)}

Diagnostic characters of the juvenile growth category of Tyrannosaurus rex.

These characters are unambiguously optimized on the topology and have a consistency index $(\mathrm{Cl})$ of 1.0 indicating that they have changed only once on the tree and so can be considered diagnostic of the growth category. Abbreviations: PHYLO, corresponding homologous phylogenetic character (see Supplemental Data S1). 
1

\begin{tabular}{ll}
\hline Character & Growth change \\
\hline 4. Orbital fenestra, shape (PHYLO 7) & $0==>1$ \\
\hline 27. Maxilla, maxillary flange, size (PHYLO 47) & $0==>1$ \\
\hline 57. Jugal, postorbital process, orientation (PHYLO 101) & $0==>1$ \\
\hline 66. Postorbital, jugal, ramus, subocular process, presence (PHYLO 116) & $0==>1$ \\
\hline 89. Frontal, postorbital buttress, distinctiveness (PHYLO 159) & $0==>1$ \\
\hline 123. Dentary, Meckelian groove, depth (PHYLO 236) & $0==>1$ \\
\hline 215. Bony naris, dorsal margin & $0==>1$ \\
\hline 226. Internal antorbital fenestra, jugal contribution & $0==>1$ \\
\hline 238. Postorbital bar, jugopostorbital suture & $0==>1$ \\
\hline 239. Postorbital bar, orientation & $0==>1$ \\
\hline 240. Postorbital bar, plane change & $0==>1$ \\
\hline 254. Premaxilla, body, rostral margin, angulation position & $0==>1$ \\
\hline 293. Nasal, cross-section of rostral third, shape & $0==>1$ \\
\hline 296. Nasal, midline bumps, number & $1==>2$ \\
\hline 299. Nasal, dorsal foramina, number rows & $1==>2$ \\
\hline 315. Nasal, frontal ramus, dorsum, cross section & $0==>1$ \\
\hline 358. Maxilla, subcutaneous surface, orientation to ventral margin & $0==>1$ \\
\hline 415. Maxilla, palatal process, extent of bony choana & $0==>1$ \\
\hline 556. Jugal, body, degree of texture & $0==>1$ \\
\hline 621. Postorbital, body, medial rugosities & $0==>1$ \\
\hline 1069. Dentary, bone, shallowest point & $0==>1$ \\
\hline 1170. Prearticular, caudal ramus, dorsal \& ventral margins, orientation & $0==>1$ \\
\hline 1173. Prearticular, rostral ramus, form & $0==>1$ \\
\hline 1176. Dentition, frst maxllary tooth, form & $0==>1$ \\
\hline & \\
\hline
\end{tabular}




\section{Table 7(on next page)}

Diagnostic characters of the subadult growth category of Tyrannosaurus rex.

These characters are unambiguously optimized on the topology and have a consistency index $(\mathrm{Cl})$ of 1.0 indicating that they have changed only once on the tree and so can be considered diagnostic of the growth category. Abbreviations: PHYLO, corresponding homologous phylogenetic character (see Supplemental Data S1). 


\begin{tabular}{|c|c|}
\hline Character & $\begin{array}{l}\text { Growth } \\
\text { change }\end{array}$ \\
\hline 37. Lacrimal, bone, shape (PHYLO 65) & $0==>1$ \\
\hline 38. Lacrimal, cornual process, apex, shape (PHYLO 66) & $1==>2$ \\
\hline 40. Lacrimal, height above lacrimal pneumatic recess (PHYLO 69) & $0==>1$ \\
\hline 41. Lacrimal, rostral ramus, inflation (PHYLO 75) & $0==>1$ \\
\hline 41. Lacrimal, rostral ramus, inflation (PHYLO 75) & $1==>2$ \\
\hline 43. Lacrimal, number of recesses ahead of lacrimal pneumatic recess (PHYLO 78) & $0==>1$ \\
\hline 44. Lacrimal, medial pneumatic recess, presence (PHYLO 79) & $0==>1$ \\
\hline 45. Lacrimal, supraorbital ramus, length (PHYLO 82) & $0==>1$ \\
\hline 46. Lacrimal, supraorbital process, inflation (PHYLO 82) & $0==>1$ \\
\hline 219. Snout, dorsum, width caudal end & $0==>1$ \\
\hline 433. Lacrimal, dorsal ramus, medial edge, form & $0==>1$ \\
\hline 434. Lacrimal, medial fossa, rostral ridge, presence & $0==>1$ \\
\hline 436. Lacrimal, dorsal ramus, conchal surface, dorsal ridge, depth of caudal part & $0==>1$ \\
\hline 447. Lacrimal, dorsal ramus, region dorsal \& rostrodorsal to lacrimal recess, form & $0==>1$ \\
\hline 454. Lacrimal, cornual process, lateral extent & $0==>1$ \\
\hline 455. Lacrimal, lacrimal pneumatic recess, foramen in ventral margin, presence & $0==>1$ \\
\hline 461. Lacrimal, region caudal to lacrimal pneumatic recess, length $\&$ depth & $0==>1$ \\
\hline 462. Lacrimal, length lacrimal pneumatic recess relative to region behind it & $0==>1$ \\
\hline 465. Lacrimal, rostral ramus, subcutaneous surface height & $0==>1$ \\
\hline 466. Lacrimal, junction antorbital fossa $\&$ subcutaneous surface ahead of recess & $0==>1$ \\
\hline 468. Lacrimal, region between lacrimal pneumatic recess septum \& distal recess & $0==>1$ \\
\hline 470. Lacrimal, pneumatic recess, proximity to maxilla & $0==>1$ \\
\hline 474. Lacrimal, rostral ramus, medial joint surfaces, height & $0==>1$ \\
\hline 476. Lacrimal, medial tab, dorsal margin & $0==>1$ \\
\hline 481. Lacrimal, maxillary process, height & $0==>1$ \\
\hline 482. Lacrimal, caudolateral shelf, form & $1==>2$ \\
\hline 489. Lacrimal, frontal process, groove on dorsal surface, presence & $0==>1$ \\
\hline 517. Lacrimal, ventral ramus, proximal to distal ends & $0==>1$ \\
\hline 524. Lacrimal, ventral ramus, subcutaneous surface, caudal extension & $0==>1$ \\
\hline 1546. Scapulocoracoid, acromial region, lateral surface, form & $0==>1$ \\
\hline 1548. Scapula, glenoid, width joint surface relative to shaft & $0==>1$ \\
\hline 1549. Scapula, glenoid, anterolateral corner, form & $0==>1$ \\
\hline 1550. Scapula, lip of glenoid, emergence form ventral edge, form & $0==>1$ \\
\hline 1551. Scapula, acromion, medial surface, form & $0==>1$ \\
\hline 1561. Scapula, muscle scar along dorsal edge, distinctiveness & $0==>1$ \\
\hline 1568. Scapula, shaft, ventral surface adjacent to glenoid, orientation & $0==>1$ \\
\hline 1574. Coracoid, glenoid, orientation & $0==>1$ \\
\hline 1576. Coracoid, glenoid, separation from coracoid process, distinctiveness & $0==>1$ \\
\hline 1577. Coracoid, low ridge that extends anteriorly form biceps tubercle, presence & $0==>1$ \\
\hline 1581. Coracoid, ventral half of bone, form & $0==>1$ \\
\hline 1583. Coracoid process, length, depth \& curvature & $0==>1$ \\
\hline 1584. Coracoid, medial fossa between coracoid foramen \& an & $0==>1$ \\
\hline
\end{tabular}




\begin{tabular}{ll}
\hline 1707. D II, PH 2, proximal joint surface, ventral margin, form & $0==>1$ \\
\hline 1711. D II, PH 2, lateral distal condyle, orientation, lateral view & $0==>1$ \\
\hline 1712. D II, PH 2, medial distal condyle, elevation & $0==>1$ \\
\hline 1714. D II, PH 2, proximodorsal flange, width & $0==>1$ \\
\hline 1715. D II, PH 2, medial distal condyle, medial margin, orientation & $0==>1$ \\
\hline 1719. D II, PH 2, medial distal condyle, ventral extent & $0==>1$ \\
\hline 1748. D III, PH 1, proximal joint surface, form & $0==>1$ \\
\hline 1754. D III, PH 1, lateral caudolateral ligament pit, frm & $0==>1$ \\
\hline 1779. D IV, PH 1, supracondylar pit, form & $0==>1$ \\
\hline 1782. D IV, PH 1, ventromedial condyle, angle relative to shaft & $0==>1$ \\
\hline 1784. D IV, PH 1, proximal joint surface, ventral notch, mediolateral position & $0==>1$ \\
\hline 1787. D IV, PH 1, distal joint surface, proportions & $0==>1$ \\
\hline 1790. D IV, PH 1, scar on shaft ahead of proximal end, prominence & $0==>1$ \\
\hline 1792. D IV, PH 1, dorsolateral surface, form & $0==>1$ \\
\hline 1793. D IV, PH 1, shaft, ridge lateral to supracondylar pit, form & $0==>1$ \\
\hline 1806. D IV, PH 3, form & $0==>1$ \\
\hline 1809. D IV, PH 3, ratio of mediolateral width to dorsoventral height & $0==>1$ \\
\hline 1810. D IV, PH 3, proximal joint surface, medial margin, form & $0==>1$ \\
\hline 1812. D IV, PH 3, medial collateral ligament point, depth & $0==>1$ \\
\hline 1814. D IV, PH 3, distal condyles, divergence & $0==>1$ \\
\hline 1815. D IV, PH 3, flexor muscle scar, form & $0==>1$ \\
\hline 1817. D IV PH 4, dorsum, form & $0==>1$ \\
\hline 1818. D IV PH 4, dorsum, mediolateral orientation & $0==>1$ \\
\hline 1820. D IV PH 4, distal joint surface, form & $0==>1$ \\
\hline 1824. D IV PH 4, distal joint surface, dorsal margin, indentation, presence & $0==>1$ \\
\hline 1826. D IV PH 4, distal condylar region, posterior margin, undercut & $0==>1$ \\
\hline 1848. Growth rings, number & $2==>3$ \\
\hline
\end{tabular}




\section{Table 8 (on next page)}

Diagnostic characters of the young adult growth category of Tyrannosaurus rex.

These characters are unambiguously optimized on the topology and have a consistency index $(\mathrm{Cl})$ of 1.0 indicating that they have changed only once on the tree and so can be considered diagnostic of the growth category. Abbreviations: PHYLO, corresponding homologous phylogenetic character (see Supplemental Data S1). 
1

\begin{tabular}{ll}
\hline Character & Growth change \\
\hline 18. Maxilla, interfenestral strut, length (PHYLO 31) & $0==>1$ \\
\hline 24. Maxilla, alveolar process, subcutaneous surface, fossae (PHYLO 44) & $0==>1$ \\
\hline 109. Otoccipital, paroccipital process, caudal surface, form (PHYLO 200) & $0==>1$ \\
\hline 253. Premaxilla, lateral margin, form & $0==>1$ \\
\hline 376. Maxilla, antorbital fossa, mediolateral depth & $0==>1$ \\
\hline 523. Lacrimal, subcutaneous surface, texture & $0==>1$ \\
\hline 651. Postorbital, jugal ramus, width, caudal & $0==>1$ \\
\hline 717. Squamosal, dorsotemporal fossa, lateral half, texture & $0==>1$ \\
\hline 737. Quadratojugal, squamosal process, ridge, width & $0==>1$ \\
\hline 741. Quadratojugal, dorsal joint surface for quadratojugal, texture \& depth & $0==>1$ \\
\hline 742. Quadratojugal, dorsal joint surface for quadrate, rostral margin, form & $0==>1$ \\
\hline 882. Frontals, proportions apposed bones & $0==>1$ \\
\hline 913. Frontal, joint surface for the prefrontal, rostrocaudal position & $0==>1$ \\
\hline 931. Frontal, orbital surface, medial margin, orientation & $1==>2$ \\
\hline 1014. Otoccipital, metotic strut, oval scar complex, participation & $0==>1$ \\
\hline 1017. Subcondylar recesses, distance from each other & $0==>1$ \\
\hline 1043. Basisphenoid, oval scar, texture & $1==>2$ \\
\hline 1048. Basisphenoid, spout, presence & $0==>1$ \\
\hline 1052. Basisphenoid, flange form ventral end of preotic pendant & $0==>1$ \\
\hline 1055. Supraoccipital, bar across lateral processes, prominence & $0==>1$ \\
\hline 1082. Dentary, chin, subcutaneous surface, texture & $0==>1$ \\
\hline 111. Dentary, ventral bar, depth below Meckelian fossa & $0==>1$ \\
\hline 1168. Angular, 2ndry ridge, form & $0==>1$ \\
\hline 1647. Fibula, ratio of midheight length to total height & $0==>1$ \\
\hline 1657. Fibula, proximal end, dorsal margin, form & $0==>1$ \\
\hline 1672. Fibula, anterior surface proximal surface to bipartite scar & $1==>2$ \\
\hline 1675. Fibula, medial fossa, differentiation & $0==>1$ \\
\hline 16.83. Fibula, ventral end below bipartite scar, form & $0==>1$ \\
\hline
\end{tabular}




\section{Table 9 (on next page)}

Diagnostic characters of the adult and senescent growth categories of Tyrannosaurus rex.

These characters are unambiguously optimized on the topology and have a consistency index $(\mathrm{Cl})$ of 1.0 indicating that they have changed only once on the tree and so can be considered diagnostic of the growth category. A double line separates the adult category (11 characters) from the senescent adult character. Abbreviations: PHYLO, corresponding homologous phylogenetic character (see Supplemental Data S1). 
1

\begin{tabular}{ll}
\hline Character & $\begin{array}{l}\text { Growth } \\
\text { change }\end{array}$ \\
\hline 147. Cervical vertebrae, centrum, hypapophysis, presence (PHYLO 280) & $0==>1$ \\
\hline 166. Ilium, pubic peduncle, ventral margin, orientation (PHYLO 333) & $0==>1$ \\
\hline 546. Jugal, medial maxillary process, rostral end, depth inset & $1==>2$ \\
\hline 590. Jugal, ventral quadratojugal process, dorsolateral surface & $1==>2$ \\
\hline 671. Squamosal, quadratojugal process, ventral margin, concavity & $0==>1$ \\
\hline 694. Squamosal, joint surface for otoccipital, form & $0==>1$ \\
\hline 735. Quadratojugal, jugal ramus, ventral margin, form & $1==>2$ \\
\hline 1051. Basisphenoid, lateral margin, form & $0==>1$ \\
\hline 1223. Axis, axial intercentrum, joint surface, ratio height to mediolateral width & $0==>1$ \\
\hline 1256. Axis, spinous process, dorsolateral process, dorsoventral height & $0==>1$ \\
\hline 1613. Ischium, semicircular scars, number & $0==>1$ \\
\hline 1668. Fibula, distal end, anterior margin, form & $0==>1$ \\
\hline 1849. EFS, presence & $0==>1$ \\
\hline \hline 1220. Axis, axial intercentrum, anterior joint surface, ventral margin, separation & $1==>2$ \\
\hline
\end{tabular}




\section{Table $\mathbf{1 0}$ (on next page)}

Data used in the correlation test of bite force and maturity in Tyrannosaurus rex.

The data for BMRP 2002.4.1are from Bates and Falkingham (2012) and the data for the others are from Gignac and Erickson (2017). Data used in the correlation test are in boldface. 
1

\begin{tabular}{lllll}
\hline Specimen & Growth rank & $\begin{array}{l}\text { Revised growth } \\
\text { rank }\end{array}$ & Bite force & $\begin{array}{l}\text { Bite force } \\
\text { rank }\end{array}$ \\
\hline BMRP 2002.4.1 & 4 & $\mathbf{1}$ & 2,400 to $3,850 \mathrm{~N}$ & $\mathbf{1}$ \\
\hline TMP 1981.006.0001 & 8 & $\mathbf{2}$ & 12,197 to $21,799 \mathrm{~N}$ & $\mathbf{2}$ \\
\hline MOR 980 & 16 & $\mathbf{3}$ & 14,201 to 30,487 N & $\mathbf{4}$ \\
\hline LACM 23844 & 17 & $\mathbf{4}$ & 16,352 to 31,284 N & $\mathbf{5}$ \\
\hline MOR 008 & 18 & $\mathbf{5}$ & 13,736 to $28,101 \mathrm{~N}$ & $\mathbf{3}$ \\
\hline FMNH PR2081 & 20 & $\mathbf{6}$ & 17,769 to 34,522 N & $\mathbf{6}$ \\
\hline
\end{tabular}

2 


\section{Table $\mathbf{1 1}$ (on next page)}

Summary of width to length ratios of maxillary and dentary teeth in adult Tyrannosaurus rex compared with representative adults of other tyrannosaurids.

In $T$. rex the maxillary teeth tend to be wider than in other tyrannosaurids, and also at the mesial end of the tooth row of the dentary. However, the characterization by Osborn (1906) of the teeth in $T$. rex as generally wider than long is not supported by these data. 


\begin{tabular}{|c|c|c|c|c|c|c|c|c|c|c|}
\hline & T. rex & & & & & & & & $D$ & \\
\hline & MOR & RSM & LACM & MOR & $\mathrm{CM}$ & T. rex & BMRP & CMN & MOR & TMP \\
\hline & 008 & 2523.8 & 23844 & 980 & 9380 & mean & 2002.4 .1 & 8506 & 1130 & $\begin{array}{l}1981.010 .000 \\
1\end{array}$ \\
\hline Mx1 & - & $85 \%$ & $71 \%$ & - & - & $78 \%$ & $?, 150 \%$ & $87 \%$ & - & - \\
\hline $\mathrm{Mx} 2$ & - & - & - & $91 \%$ & - & $91 \%$ & $73 \%, 83 \%$ & $64 \%$ & $79 \%$ & $56 \%$ \\
\hline Mx3 & - & $65 \%$ & $69 \%$ & - & - & $67 \%$ & $48 \%, ?$ & - & - & $68 \%$ \\
\hline $\mathrm{Mx} 4$ & $98 \%$ & - & - & - & - & $98 \%$ & $? 54 \%$ & $100 \%$ & - & $59 \%$ \\
\hline $\mathrm{Mx} 5$ & - & $77 \%$ & $72 \%$ & - & - & $74.5 \%$ & $50 \%, ?$ & - & - & $56 \%$ \\
\hline Mx6 & - & - & $71 \%$ & $85 \%$ & - & $78 \%$ & $50 \%, 56 \%$ & $74 \%$ & - & $63 \%$ \\
\hline $\mathrm{Mx} 7$ & $74 \%$ & $81 \%$ & - & $85 \%$ & - & $80 \%$ & $45 \%, ?$ & - & - & $63 \%$ \\
\hline Mx8 & $76 \%$ & - & - & - & - & $76 \%$ & $53 \%, 51 \%$ & $69 \%$ & - & $61 \%$ \\
\hline Mx9 & $84 \%$ & - & - & - & - & $84 \%$ & $54 \%, ?$ & - & - & - \\
\hline Mx10 & $84 \%$ & $72 \%$ & - & - & $77 \%$ & $77.7 \%$ & $?, 54 \%$ & $73 \%$ & - & - \\
\hline Mx11 & $84 \%$ & - & - & - & - & $84 \%$ & $?, 50 \%$ & $72 \%$ & - & - \\
\hline Mx12 & - & - & $\mathrm{n} / \mathrm{a}$ & - & - & - & $47 \%, ?$ & - & - & $63 \%$ \\
\hline Mx13 & $\mathrm{n} / \mathrm{a}$ & $\mathrm{n} / \mathrm{a}$ & $\mathrm{n} / \mathrm{a}$ & $\mathrm{n} / \mathrm{a}$ & $\mathrm{n} / \mathrm{a}$ & - & $49 \%, 51 \%$ & - & - & - \\
\hline Mx14 & $\mathrm{n} / \mathrm{a}$ & $\mathrm{n} / \mathrm{a}$ & $\mathrm{n} / \mathrm{a}$ & $\mathrm{n} / \mathrm{a}$ & $\mathrm{n} / \mathrm{a}$ & - & $50 \%, ?$ & - & - & - \\
\hline Mx15 & $\mathrm{n} / \mathrm{a}$ & $\mathrm{n} / \mathrm{a}$ & $\mathrm{n} / \mathrm{a}$ & $\mathrm{n} / \mathrm{a}$ & $\mathrm{n} / \mathrm{a}$ & - & $54 \%, 53 \%$ & - & - & - \\
\hline Mx16 & $\mathrm{n} / \mathrm{a}$ & $\mathrm{n} / \mathrm{a}$ & $\mathrm{n} / \mathrm{a}$ & $\mathrm{n} / \mathrm{a}$ & $\mathrm{n} / \mathrm{a}$ & - & $?, ?$ & - & - & - \\
\hline Dn1 & - & $170 \%$ & - & - & $140 \%$ & $155 \%$ & $\begin{array}{l}130 \%, \\
120 \%\end{array}$ & - & - & - \\
\hline Dn2 & - & $110 \%$ & $73 \%$ & - & $94 \%$ & $92.3 \%$ & $83 \%, 84 \%$ & - & - & - \\
\hline Dn3 & - & - & - & - & $88 \%$ & $88 \%$ & $?, 70 \%$ & - & - & - \\
\hline Dn4 & $82 \%$ & $87 \%$ & - & - & $84 \%$ & $84.3 \%$ & $64 \%, 65 \%$ & $78 \%$ & - & - \\
\hline Dn5 & - & $120 \%$ & $73 \%$ & - & $77 \%$ & $90 \%$ & $58 \%, ?$ & $70 \%$ & - & - \\
\hline Dn6 & $83 \%$ & - & - & - & - & $83 \%$ & $61 \%, 58 \%$ & - & - & - \\
\hline Dn7 & $79 \%$ & $130 \%$ & $76 \%$ & - & $82 \%$ & $91.8 \%$ & $?, 54 \%$ & $90 \%$ & - & - \\
\hline Dn8 & - & $79 \%$ & - & - & - & $76 \%$ & $53 \%, ?$ & - & - & - \\
\hline Dn9 & $77 \%$ & - & - & - & - & $77 \%$ & $53 \%, ?$ & $80 \%$ & - & - \\
\hline Dn10 & $69 \%$ & - & - & - & $76 \%$ & $72.5 \%$ & $57 \%, ?$ & $86 \%$ & - & - \\
\hline Dn11 & $69 \%$ & - & - & - & - & $69 \%$ & $53 \%, ?$ & $74 \%$ & - & - \\
\hline Dn12 & $70 \%$ & - & $67 \%$ & - & $76 \%$ & $71 \%$ & $?$ & - & - & - \\
\hline Dn13 & $80 \%$ & $60 \%$ & $\mathrm{n} / \mathrm{a}$ & - & - & $70 \%$ & $50 \%, ?$ & $75 \%$ & - & - \\
\hline Dn14 & $\mathrm{n} / \mathrm{a}$ & $79 \%$ & $\mathrm{n} / \mathrm{a}$ & - & $\mathrm{n} / \mathrm{a}$ & $79 \%$ & $50 \%, ?$ & $72 \%$ & - & - \\
\hline Dn15 & $\mathrm{n} / \mathrm{a}$ & $\mathrm{n} / \mathrm{a}$ & $\mathrm{n} / \mathrm{a}$ & - & $\mathrm{n} / \mathrm{a}$ & - & $58 \%, ?$ & - & - & - \\
\hline Dn16 & $\mathrm{n} / \mathrm{a}$ & $\mathrm{n} / \mathrm{a}$ & $\mathrm{n} / \mathrm{a}$ & - & $\mathrm{n} / \mathrm{a}$ & - & $53 \%, ?$ & $83 \%$ & - & - \\
\hline Dn17 & $\mathrm{n} / \mathrm{a}$ & $\mathrm{n} / \mathrm{a}$ & $\mathrm{n} / \mathrm{a}$ & - & $\mathrm{n} / \mathrm{a}$ & - & $65 \%, ?$ & $\mathrm{n} / \mathrm{a}$ & - & - \\
\hline
\end{tabular}




\section{Table 12 (on next page)}

Summary of maxillary tooth count data and growth rank in Tyrannosaurus rex.

Summary of raw and ranked data used for the Spearman correlation test between maturity and maxillary tooth count in T. rex. 
1

\begin{tabular}{lllllll}
\hline Specimen & $\begin{array}{l}\text { Maxillary } \\
\text { tooth \# }\end{array}$ & $\begin{array}{l}\text { Maxillary } \\
\text { tooth } \# \\
\text { rank }\end{array}$ & $\begin{array}{l}\text { Maxillary } \\
\text { tooth } \\
\text { midranks }\end{array}$ & $\begin{array}{l}\text { Growth } \\
\text { stage }\end{array}$ & $\begin{array}{l}\text { Growth } \\
\text { stage } \\
\text { rank }\end{array}$ & $\begin{array}{l}\text { Growth } \\
\text { stage } \\
\text { midranks }\end{array}$ \\
\hline CMNH 741 & 15 & 2 & $\mathbf{2}$ & 3 & 1 & $\mathbf{1}$ \\
\hline BMRP 2002.4.1 & 16 & 1 & $\mathbf{1}$ & 4 & 2 & $\mathbf{2}$ \\
\hline MOR 1125 & 12 & 3 & $\mathbf{7}$ & 7 & 3 & $\mathbf{3}$ \\
\hline TMP 1981.006.0001 & 12 & 4 & $\mathbf{7}$ & 8 & 4 & $\mathbf{4}$ \\
\hline UWBM 99000 & 12 & 5 & $\mathbf{7}$ & 11 & 5 & $\mathbf{5}$ \\
\hline RSM 2523.8 & 11 & 12 & $\mathbf{1 3}$ & 12 & 6 & $\mathbf{6 . 5}$ \\
\hline SDSM 12047 & 12 & 6 & $\mathbf{7}$ & 12 & 7 & $\mathbf{6 . 5}$ \\
\hline AMNH FARB 5027 & 12 & 7 & $\mathbf{7}$ & 13 & 8 & $\mathbf{8}$ \\
\hline MOR 555 & 12 & 8 & $\mathbf{7}$ & 16 & 9 & $\mathbf{9 . 5}$ \\
\hline MOR 980 & 11 & 13 & $\mathbf{1 3}$ & 16 & 10 & $\mathbf{9 . 5}$ \\
\hline LACM 23844 & 11 & 14 & $\mathbf{1 3}$ & 17 & 11 & $\mathbf{1 1 . 5}$ \\
\hline CM 9380 & 12 & 9 & $\mathbf{7}$ & 17 & 12 & $\mathbf{1 1 . 5}$ \\
\hline MOR 008 & 12 & 10 & $\mathbf{7}$ & 18 & 13 & $\mathbf{1 3}$ \\
\hline FMNH PR2081 & 12 & 11 & $\mathbf{7}$ & 20 & 14 & $\mathbf{1 4}$ \\
\hline
\end{tabular}

2

3 


\section{Table 13 (on next page)}

Summary of dentary tooth count data and growth rank in Tyrannosaurus rex.

Summary of raw and ranked data used in the Spearman rank correlation test of maturity and dentary tooth count in T. rex. 
1

\begin{tabular}{lllllll}
\hline Specimen & $\begin{array}{l}\text { Dentary } \\
\text { tooth \# }\end{array}$ & $\begin{array}{l}\text { Dentary } \\
\text { tooth } \# \\
\text { rank }\end{array}$ & $\begin{array}{l}\text { Dentary } \\
\text { tooth \# } \\
\text { midranks }\end{array}$ & $\begin{array}{l}\text { Growth } \\
\text { stage }\end{array}$ & $\begin{array}{l}\text { Growth } \\
\text { rank }\end{array}$ & $\begin{array}{l}\text { Growth } \\
\text { midranks }\end{array}$ \\
\hline CMNH 7541 & 16 & 2 & $\mathbf{2}$ & 3 & 1 & $\mathbf{1}$ \\
\hline BMRP 2002.4.1 & 17 & 1 & $\mathbf{1}$ & 4 & 2 & $\mathbf{2}$ \\
\hline MOR 1125 & 14 & 3 & $\mathbf{5}$ & 7 & 3 & $\mathbf{3}$ \\
\hline TMP 1981.006.0001 & 13 & 8 & $\mathbf{1 1 . 5}$ & 8 & 4 & $\mathbf{4}$ \\
\hline LACM 150167 & 13 & 9 & $\mathbf{1 1 . 5}$ & 9 & 5 & $\mathbf{5}$ \\
\hline UWBM 99000 & 13 & 10 & $\mathbf{1 1 . 5}$ & 11 & 6 & $\mathbf{6}$ \\
\hline RSM 2523.8 & 14 & 4 & $\mathbf{5}$ & 12 & 7 & $\mathbf{7 . 5}$ \\
\hline SDSM 12047 & 14 & 5 & $\mathbf{5}$ & 12 & 8 & $\mathbf{7 . 5}$ \\
\hline AMNH FARB 5027 & 14 & 6 & $\mathbf{5}$ & 13 & 9 & $\mathbf{9}$ \\
\hline BM R7994 & 14 & 7 & $\mathbf{5}$ & 14 & 10 & $\mathbf{1 0}$ \\
\hline NMMNH P-3698 & 13 & 11 & $\mathbf{1 1 . 5}$ & 15 & 11 & $\mathbf{1 1}$ \\
\hline MOR 555 & 12 & 16 & $\mathbf{1 6 . 5}$ & 16 & 12 & $\mathbf{1 2 . 5}$ \\
\hline MOR 980 & 13 & 12 & $\mathbf{1 1 . 5}$ & 16 & 13 & $\mathbf{1 2 . 5}$ \\
\hline LACM 23844 & 12 & 17 & $\mathbf{1 6 . 5}$ & 17 & 14 & $\mathbf{1 4 . 5}$ \\
\hline CM 9380 & 13 & 13 & $\mathbf{1 1 . 5}$ & 17 & 15 & $\mathbf{1 4 . 5}$ \\
\hline MOR 008 & 13 & 14 & $\mathbf{1 1 . 5}$ & 18 & 16 & $\mathbf{1 6}$ \\
\hline FMNH PR2081 & 13 & 15 & $\mathbf{1 1 . 5}$ & 20 & 17 & $\mathbf{1 7}$ \\
\hline
\end{tabular}


Table 14(on next page)

Summary of chronological age data and growth rank in Tyrannosaurus rex.

Summary of the raw and ranked data used in the Spearman correlation test of maturity and chronological age in T. rex. 
1

\begin{tabular}{llllll}
\hline Specimen & $\begin{array}{l}\text { Chronological } \\
\text { age }\end{array}$ & $\begin{array}{l}\text { Chronological } \\
\text { age rank }\end{array}$ & $\begin{array}{l}\text { Chronological } \\
\text { age midranks }\end{array}$ & $\begin{array}{l}\text { Growth stage } \\
\text { Growth stage } \\
\text { rank }\end{array}$ \\
\hline LACM 28471 & 2 & 1 & $\mathbf{1}$ & 1 & $\mathbf{1}$ \\
\hline BMRP 2002.4.1 & 13 & 2 & $\mathbf{2}$ & 4 & $\mathbf{2}$ \\
\hline BMRP 2006.4.4 & 15 & 4 & $\mathbf{4}$ & 5 & $\mathbf{3}$ \\
\hline LACM 23845 & 14 & 3 & $\mathbf{3}$ & 6 & $\mathbf{4}$ \\
\hline MOR 1125 & 18 & 5 & $\mathbf{5 . 5}$ & 7 & $\mathbf{6}$ \\
\hline TMP 1981.006.0001 & 18 & 6 & $\mathbf{5 . 5}$ & 8 & $\mathbf{7}$ \\
\hline TMP 1981.012.0001 & 22 & 7 & $\mathbf{7}$ & 11 & $\mathbf{8}$ \\
\hline FMNH PR2081 & 28 & 8 & $\mathbf{8}$ & 20 & \\
\hline
\end{tabular}

2 


\section{Table 15(on next page)}

Summary of size data and growth rank in Tyrannosaurus rex.

Summary of raw and ranked data used in the Spearman correlation test of size rank and growth rank in T. rex. 
1

\begin{tabular}{lllllll}
\hline Specimen & $\begin{array}{l}\text { Size } \\
(\mathrm{cm})\end{array}$ & Size rank & $\begin{array}{l}\text { Size } \\
\text { midranks }\end{array}$ & $\begin{array}{l}\text { Growth } \\
\text { stage }\end{array}$ & $\begin{array}{l}\text { Growth } \\
\text { stage } \\
\text { rank }\end{array}$ & $\begin{array}{l}\text { Growth } \\
\text { stage } \\
\text { midranks }\end{array}$ \\
\hline LACM 28471 & 40 & 1 & $\mathbf{1}$ & 1 & 1 & $\mathbf{1}$ \\
\hline CMNH 7541 & 57 & 2 & $\mathbf{2}$ & 3 & 2 & $\mathbf{2}$ \\
\hline BMRP 2002.4.1 & 74 & 3 & $\mathbf{3}$ & 4 & 3 & $\mathbf{3}$ \\
\hline LACM 23845 & 80 & 4 & $\mathbf{4}$ & 6 & 4 & $\mathbf{4}$ \\
\hline MOR 1125 & 116 & 5 & $\mathbf{5}$ & 7 & 5 & $\mathbf{5}$ \\
\hline TMP 1981.006.0001 & 120 & 6 & $\mathbf{6 . 5}$ & 8 & 6 & $\mathbf{6}$ \\
\hline LACM 150167 & 120 & 7 & $\mathbf{6 . 5}$ & 9 & 7 & $\mathbf{7}$ \\
\hline UWBM 99000 & 130 & 8 & $\mathbf{8 . 5}$ & 11 & 8 & $\mathbf{8}$ \\
\hline RSM 2523.8 & 140 & 12 & $\mathbf{1 3 . 5}$ & 12 & 9 & $\mathbf{9}$ \\
\hline AMNH FARB 5027 & 136 & 10 & $\mathbf{1 0 . 5}$ & 13 & 10 & $\mathbf{1 0}$ \\
\hline MOR 980 & 136 & 11 & $\mathbf{1 0 . 5}$ & 16 & 11 & $\mathbf{1 1 . 5}$ \\
\hline LACM 23844 & 140 & 14 & $\mathbf{1 3 . 5}$ & 16 & 12 & $\mathbf{1 1 . 5}$ \\
\hline CM 9380 & 130 & 9 & $\mathbf{8 . 5}$ & 17 & 13 & $\mathbf{1 3 . 5}$ \\
\hline MOR 008 & 140 & 13 & $\mathbf{1 3 . 5}$ & 17 & 14 & $\mathbf{1 3 . 5}$ \\
\hline FMNH PR2081 & 140 & 15 & $\mathbf{1 3 . 5}$ & 20 & 15 & $\mathbf{1 5}$ \\
\hline
\end{tabular}




\section{Table $\mathbf{1 6}$ (on next page)}

Summary of mass data and growth rank for Tyrannosaurus rex.

Summary of raw and ranked data for the Spearman correlation test of mass and maturity in

T. rex. Masses are drawn from Erickson et al (2004) and Persons et al. (2019). 
1

\begin{tabular}{llllll}
\hline Specimen & Mass $(\mathrm{kg})$ & Mass rank & $\begin{array}{l}\text { Growth } \\
\text { stage }\end{array}$ & $\begin{array}{l}\text { Growth } \\
\text { stage rank }\end{array}$ & $\begin{array}{l}\text { Growth } \\
\text { stage } \\
\text { midranks }\end{array}$ \\
\hline LACM 28471 & 25 & 1 & 1 & 1 & $\mathbf{1}$ \\
\hline LACM 23845 & 98.9 & 2 & 6 & 2 & $\mathbf{2}$ \\
\hline MOR 1125 & 6,100 & 5 & 7 & 3 & $\mathbf{3}$ \\
\hline TMP 1981.006.0001 & 4,469 & 3 & 8 & 4 & $\mathbf{4}$ \\
\hline RSM 2523.8 & 8,870 & 9 & 12 & 5 & $\mathbf{6 . 5}$ \\
\hline MOR 555 & 6,264 & 6 & 16 & 6 & $\mathbf{6 . 5}$ \\
\hline MOR 980 & 5,112 & 4 & 16 & 7 & $\mathbf{7}$ \\
\hline FM 9380 & 6,740 & 7 & 17 & 8 & $\mathbf{8}$ \\
\hline
\end{tabular}

2

3 


\section{Table $\mathbf{1 7}$ (on next page)}

Summary of data used in the correlation test between geographic latitude and maturity of Tyrannosaurus rex specimens.

For each fossil, the columns are organized by specimen number, growth stage rank, geographic location rank, and level of resolution of the locality data. Specimens are listed by descending location rank (i.e., from north to south). Abbreviations: Co, county; km, kilometer; $\mathbf{m}$, meter; $\mathbf{m i}$, miles; $\mathbf{S}$, section; asterisk $(*)$ indicates the resolution is almost certainly an underestimate. 
1

\begin{tabular}{|c|c|c|c|}
\hline Specimen number & Maturity midrank & Location midrank & Resolution \\
\hline TMP 1981.012.0001 & 12.5 & 29 & $\mathrm{~km}$ \\
\hline TMP 1981.006.0001 & 9 & 28 & $\mathrm{~km}$ \\
\hline RSM 2990.1 & 5.5 & 27 & $\mathrm{~m}$ \\
\hline RSM 2523.8 & 15 & 26 & $\mathrm{~m}$ \\
\hline CM 9380 & 22.5 & 25 & $\mathrm{~S}$ \\
\hline LACM 28471 & 1 & 24 & $\mathrm{~S}$ \\
\hline LACM 23844 & 22.5 & 22.5 & $\mathrm{~S}$ \\
\hline LACM 23845 & 7 & 22.5 & $\mathrm{~S}$ \\
\hline MOR 980 & 20 & 21 & $\mathrm{~m}$ \\
\hline UWBM 99000 & 12.5 & 20 & $\mathrm{~m}$ \\
\hline MOR 1131 & 20 & 19 & $\mathrm{~m}$ \\
\hline MOR 1125 & 8 & 18 & $\mathrm{~m}$ \\
\hline MOR 555 & 20 & 17 & $\mathrm{~m}$ \\
\hline UCMP 118742 & 25 & 16 & $\mathrm{~m}$ \\
\hline MOR 008 & 25 & 15 & $\mathrm{~m}^{*}$ \\
\hline DDM 344.1 & 2 & 14 & $\mathrm{~m}$ \\
\hline LACM 150167 & 10 & 13 & $\mathrm{~m}$ \\
\hline BMRP 2002.4.1 & 4 & 12 & $\mathrm{mi}$ \\
\hline BMRP 2006.6.4 & 5.5 & 11 & $\mathrm{mi}$ \\
\hline MOR 2822 & 27.5 & 10 & $\mathrm{~m}$ \\
\hline CMNH 7541 & 3 & 9 & $\mathrm{~S}$ \\
\hline UWGM 181 & 27.5 & 8 & $\mathrm{~S}$ \\
\hline FMNH PR2081 & 29 & 7 & $\mathrm{mi}$ \\
\hline SDSM 12047 & 15 & 6 & $\mathrm{mi}$ \\
\hline AMNH FARB 5117 & 17 & 5 & $\mathrm{Co}$ \\
\hline CM 1400 & 11 & 3.5 & $\mathrm{Co}$ \\
\hline BM R7994 & 15 & 3.5 & $\mathrm{Co}$ \\
\hline UMNH 11000 & 25 & 2 & $\mathrm{mi}$ \\
\hline NMMNH P-3698 & 18 & 1 & $\mathrm{~m}$ \\
\hline
\end{tabular}




\section{Table $\mathbf{1 8}$ (on next page)}

Summary of raw and ranked data used in the Spearman correlation test of stratigraphic level in the Hell Creek Formation (and equivalents) and maturity for Tyrannosaurus rex.

The specimens are organized by stratigraphic level and the values used in the correlation test are given in boldfaced columns; tied HCF ranks were converted to midranks.

Abbreviations: HCF, Hell Creek Formation. 
1

\begin{tabular}{lllllll}
\hline Specimen & HCF level & $\begin{array}{l}\text { HCF level } \\
\text { rank }\end{array}$ & HCF rank & $\begin{array}{l}\text { HCF level } \\
\text { midranks }\end{array}$ & $\begin{array}{l}\text { Growth } \\
\text { stage }\end{array}$ & $\begin{array}{l}\text { Growth } \\
\text { stage } \\
\text { rank }\end{array}$ \\
\hline RSM 2990.1 & Upper & 1 & 1 & $\mathbf{2}$ & 5 & $\mathbf{3}$ \\
\hline RSM 2523.8 & Upper & 1 & 2 & $\mathbf{2}$ & 12 & $\mathbf{5}$ \\
\hline MOR 555 & Upper & 1 & 3 & $\mathbf{2}$ & 17 & $\mathbf{7}$ \\
\hline DDM 344.1 & Middle & 2 & 4 & $\mathbf{4 . 5}$ & 2 & $\mathbf{1}$ \\
\hline BMRP 2002.4.1 & Middle & 2 & 5 & $\mathbf{4 . 5}$ & 4 & $\mathbf{2}$ \\
\hline NMMNH P-3698 & Lower & 3 & 6 & $\mathbf{7}$ & 15 & $\mathbf{6}$ \\
\hline MOR 1125 & Lower & 3 & 7 & $\mathbf{7}$ & 7 & $\mathbf{4}$ \\
\hline FMNH PR2081 & Lower & 3 & 8 & $\mathbf{7}$ & 20 & $\mathbf{8}$ \\
\hline
\end{tabular}




\section{Table 19 (on next page)}

Data used in the correlation test between ontogeny and phylogeny for Tyrannosaurus rex.

Phylogenetic topology of Carr et al. (2017) was followed for clade ranks. Boldfaced column gives the means for tied ranks (i.e., midranks). The category $\square$ derived tyrannosaurines $\square$ (i.e., Lythronax, Nanuqsaurus, Teratophoneus) occurs three times given the presence of three ontogenetic characters recovered in the ontogram that are homologous with corresponding synapomorphies. The $12^{\text {th }}$ growth stage occurs twice given that two synontomorphies occr at that growth stage but their homologues occur at diperent internodes on the phylogenetic hierarchy. 
1

\begin{tabular}{llllll}
\hline Clade & $\begin{array}{l}\text { Clade } \\
\text { rank }\end{array}$ & $\begin{array}{l}\text { Clade } \\
\text { midranks }\end{array}$ & $\begin{array}{l}\text { Growth } \\
\text { stage }\end{array}$ & $\begin{array}{l}\text { Growth } \\
\text { rank }\end{array}$ & $\begin{array}{l}\text { Growth } \\
\text { midranks }\end{array}$ \\
\hline 'Stokesosaurids" + derived & 1 & $\mathbf{1}$ & 9 & 7 & 7
\end{tabular}

tyrannosauroids

$\begin{array}{llllll}\text { Xiongguanlong }+ \text { derived } & 2 & \mathbf{2} & 18 & 10 & \mathbf{1 0}\end{array}$

tyrannosauroids

$\begin{array}{llllll}\text { Bistahieversor }+ \text { derived } & 3 & 3 & 5 & 2 & \mathbf{2}\end{array}$

tyrannosauroids

\begin{tabular}{llllll}
\hline Tyrannosauridae & 4 & $\mathbf{4}$ & 8 & 4 & $\mathbf{5}$ \\
\hline derived tyrannosaurines & 5 & $\mathbf{6}$ & 3 & 1 & $\mathbf{1}$ \\
\hline derived tyrannosaurines & 6 & $\mathbf{6}$ & 7 & 3 & $\mathbf{3}$ \\
\hline derived tyrannosaurines & 7 & $\mathbf{6}$ & 12 & 8 & $\mathbf{8 . 5}$ \\
\hline $\begin{array}{l}\text { Daspletosaurus }+ \text { advanced } \\
\text { tyrannosaurines }\end{array}$ & 8 & $\mathbf{8}$ & 12 & 9 & $\mathbf{8 . 5}$ \\
\hline $\begin{array}{l}\text { Zhuchengtyrannus }+ \\
\text { Tyrannosaurus }\end{array}$ & 9 & $\mathbf{9}$ & 8 & 5 & $\mathbf{5}$ \\
\hline Tyrannosaurus & 10 & $\mathbf{1 0}$ & 8 & 6 & $\mathbf{5}$ \\
\hline
\end{tabular}

2

3

4 


\section{Table 20 (on next page)}

Summary of data used in the Spearman correlation test of individual variation per specimen per node and number of synontomorphies per node for Tyrannnosaurus rex.

The rows are organized by node, followed by the sequence of number of synontomorphies and individual variation by number, rank, and midranks. Boldfaced columns indicate values used in the correlation test. 
1

\begin{tabular}{|c|c|c|c|c|c|c|}
\hline Node & $\begin{array}{l}\# \\
\text { synontomoprhi } \\
\text { es/node }\end{array}$ & $\begin{array}{l}\text { Synontomorph } \\
\text { y rank }\end{array}$ & $\begin{array}{l}\text { Synonotmorp } \\
\text { hy midrank }\end{array}$ & $\begin{array}{l}\text { Individual } \\
\text { variation/branc } \\
\mathrm{h}\end{array}$ & $\begin{array}{l}\text { individual } \\
\text { variation rank }\end{array}$ & $\begin{array}{l}\text { individual } \\
\text { variation } \\
\text { midrank }\end{array}$ \\
\hline 1 & 0 & 1 & 1.5 & 0 & 1 & 4.5 \\
\hline 2 & 2 & 4 & 5.5 & 0 & 2 & 4.5 \\
\hline 3 & 0 & 2 & 1.5 & 3 & 11 & 12 \\
\hline 4 & 3 & 8 & 8.5 & 3 & 12 & 12 \\
\hline 5 & 50 & 29 & 29 & 16 & 26 & 26 \\
\hline 6 & 90 & 30 & 30.5 & 0 & 3 & 4.5 \\
\hline 6 & 90 & 31 & 30.5 & 0 & 4 & 4.5 \\
\hline 7 & 16 & 23 & 23.5 & 6 & 18 & 18 \\
\hline 8 & 31 & 28 & 28 & 49 & 31 & 31 \\
\hline 9 & 16 & 24 & 23.5 & 6 & 18 & 18 \\
\hline 10 & 12 & 19 & 19 & 42 & 29 & 29 \\
\hline 11 & 9 & 14 & 15 & 17 & 27 & 27 \\
\hline 12 & 9 & 15 & 15 & 2 & 10 & 10 \\
\hline 12 & 9 & 16 & 15 & 0 & 5 & 4.5 \\
\hline 13 & 20 & 26 & 26.5 & 5 & 15 & 15.5 \\
\hline 13 & 20 & 27 & 26.5 & 9 & 21 & 21 \\
\hline 14 & 10 & 17 & 17.5 & 10 & 22 & 22 \\
\hline 14 & 10 & 18 & $\mathbf{1 7 . 5}$ & 5 & 16 & 15.5 \\
\hline 15 & 3 & 9 & 8.5 & 3 & 13 & 12 \\
\hline 16 & 1 & 3 & 3 & 4 & 14 & 14 \\
\hline 17 & 2 & 5 & 5.5 & 15 & 25 & 25 \\
\hline 17 & 2 & 6 & 5.5 & 41 & 28 & 28 \\
\hline 17 & 2 & 7 & 5.5 & 47 & 30 & 30 \\
\hline 18 & 6 & 10 & 10.5 & 12 & 24 & 24 \\
\hline 18 & 6 & 11 & 10.5 & 8 & 20 & 20 \\
\hline 19 & 15 & 20 & 21 & 0 & 6 & 4.5 \\
\hline 19 & 15 & 21 & 21 & 0 & 7 & 4.5 \\
\hline 19 & 15 & 22 & 21 & 1 & 9 & 9 \\
\hline 20 & 7 & 12 & 12.5 & 11 & 23 & 23 \\
\hline 20 & 7 & 13 & 12.5 & 6 & 18 & 18 \\
\hline 21 & 19 & 25 & 25 & 0 & 8 & 4.5 \\
\hline
\end{tabular}




\section{Table 21 (on next page)}

Summary of the number of synontomorphies and individual variation per craniomandibular module.

Raw and corrected data used in the correlation test between the number of synontomorphies in each skull module with the amount of individual variation per module. 
1

\begin{tabular}{llllll}
\hline Module & $\begin{array}{l}\text { Synontomorp } \\
\text { hies }\end{array}$ & $\begin{array}{l}\text { Synontomor } \\
\text { phies rank }\end{array}$ & $\begin{array}{l}\text { Individual } \\
\text { variation }\end{array}$ & $\begin{array}{l}\text { Individual } \\
\text { variation rank }\end{array}$ & $\begin{array}{l}\text { Individual } \\
\text { variation } \\
\text { midrank }\end{array}$ \\
\hline skull roof & 117 & $\mathbf{1}$ & 100 & 1 & $\mathbf{1}$ \\
\hline snout \& palate & 60 & $\mathbf{2}$ & 57 & 2 & $\mathbf{2}$ \\
\hline parietal & 12 & $\mathbf{6}$ & 23 & 5 & $\mathbf{5 . 5}$ \\
\hline suspensorium & 13 & $\mathbf{5}$ & 23 & 6 & $\mathbf{5 . 5}$ \\
\hline braincase & 25 & $\mathbf{4}$ & 29 & 3 & $\mathbf{4}$ \\
\hline mandible & 27 & $\mathbf{3}$ & 27 & 4 & $\mathbf{3}$ \\
\hline
\end{tabular}

2 


\section{Table 22 (on next page)}

Comparison of hypothetically oversplit characters in the data set for Tyrannosaurus rex ontogeny with decisions for deletion of redundant transformation series.

The rows are organized by growth stage, followed by the skull region, identi $\square$ cation of oversplit characters, and the decisions (based on redundancy) to retain (=exemplar) or exclude transformation series from the analysis. Identical transformation series have matching codings, although one might be more complete than the other. Groups of identical transformation series are separated by semicolons. Exemplar transformation series are the most completely coded examples of their sets and were run in the analysis, whereas excluded transformations series were not. 
1

\begin{tabular}{|c|c|c|c|c|c|}
\hline $\begin{array}{l}\text { Growth } \\
\text { stage }\end{array}$ & Domain & $\begin{array}{l}\text { Hypothetically } \\
\text { oversplit } \\
\text { characters }\end{array}$ & $\begin{array}{l}\text { Identical } \\
\text { transformation } \\
\text { series }\end{array}$ & $\begin{array}{l}\text { Exemplar } \\
\text { transformation } \\
\text { series }\end{array}$ & $\begin{array}{l}\text { Excluded } \\
\text { transformation } \\
\text { series }\end{array}$ \\
\hline 5 & skull height & $\begin{array}{l}4,57,66,238 \\
239,240,254, \\
260,570\end{array}$ & $\begin{array}{l}4,66 ; 570 ; 57 \\
238,239,240 \\
260\end{array}$ & $4,240,260,570$ & $66 ; 57,238,239$ \\
\hline 5 & antorbital fossa & 374,375 & $\mathrm{n} / \mathrm{a}$ & $\mathrm{n} / \mathrm{a}$ & $\mathrm{n} / \mathrm{a}$ \\
\hline 5 & $\begin{array}{l}\text { dorsal margin of } \\
\text { postorbital }\end{array}$ & 620,623 & $\mathrm{n} / \mathrm{a}$ & $\mathrm{n} / \mathrm{a}$ & $\mathrm{n} / \mathrm{a}$ \\
\hline 5 & $\begin{array}{l}\text { subcutaneous } \\
\text { surface }\end{array}$ & 556,621 & 556,621 & 621 & 556 \\
\hline 5 & $\begin{array}{l}\text { maxillary tooth } \\
\text { count }\end{array}$ & $132,397,1176$ & $\mathrm{n} / \mathrm{a}$ & $\mathrm{n} / \mathrm{a}$ & $\mathrm{n} / \mathrm{a}$ \\
\hline 6 & $\begin{array}{l}\text { lacrimal } \\
\text { inflation }\end{array}$ & $\begin{array}{l}38,40,41,45, \\
46,436,437, \\
447,450,453, \\
454,459,461, \\
462,464,465, \\
468,471,482, \\
485\end{array}$ & $\begin{array}{l}40,45,436,447, \\
454,462,465, \\
468 ; 46 ; 437, \\
450 ; 464 ; 453 ; \\
471 ; 459 ; 41, \\
461 ; 39 ; 38,482, \\
485\end{array}$ & $\begin{array}{l}447 ; 46 ; 450 ; \\
464 ; 453 ; 471 ; \\
459 ; 41 ; 39 ; 38\end{array}$ & $\begin{array}{l}40,45,436,454, \\
462,465,468 ; \\
437 ; 461 ; 482, \\
485\end{array}$ \\
\hline 6 & skull frame & $517,522,626$ & 517,$626 ; 522$ & $517 ; 522$ & 626 \\
\hline 7 & $\begin{array}{l}\text { lacrimal } \\
\text { inflation }\end{array}$ & $39,41,43,453$ & $\mathrm{n} / \mathrm{a}$ & $\mathrm{n} / \mathrm{a}$ & $\mathrm{n} / \mathrm{a}$ \\
\hline 9 & alveolus size & 117,121 & $\mathrm{n} / \mathrm{a}$ & $\mathrm{n} / \mathrm{a}$ & $\mathrm{n} / \mathrm{a}$ \\
\hline
\end{tabular}




\section{Table 23 (on next page)}

Summary of the character evidence for shared ontogenetic trends throughout the ontogeny of Archosauriformes.

Rows correspond to major growth trends identi $\square$ ed in the left hand column and, in the following cells, the osteological correlates of the trend for each taxon; columns correspond to taxa. The taxonomic distribution of the reduction of cephalic ornamentation is given in the text. Abbreviations: emf, external mandibular fenestra. See text for details and sources; see Fig. 33 for an illustrated summary. 


\begin{tabular}{|c|c|c|c|c|c|c|c|c|}
\hline $\begin{array}{l}\text { character/t } \\
\text { axon }\end{array}$ & $\begin{array}{l}\text { Proterosu } \\
\text { chus }\end{array}$ & Crocodylia & $\begin{array}{l}\text { Massospo } \\
\text { ndylus }\end{array}$ & $\begin{array}{l}\text { Coelop } \\
\text { hysis }\end{array}$ & $\begin{array}{l}\text { Dubreuillos } \\
\text { aurus }\end{array}$ & Allosaurus & $\begin{array}{l}\text { Tyrannos } \\
\text { aurus } \\
\text { bataar }\end{array}$ & T. rex \\
\hline $\begin{array}{l}\text { increase } \\
\text { in snout } \\
\text { height }\end{array}$ & $\begin{array}{l}\text { height } \\
\text { maxillary } \\
\text { horizontal } \\
\text { ramus }\end{array}$ & $\begin{array}{l}\text { snout } \\
\text { height }\end{array}$ & $\begin{array}{l}\text { snout } \\
\text { height }\end{array}$ & $?$ & $\begin{array}{l}\text { snout } \\
\text { height }\end{array}$ & $\begin{array}{l}\text { snout } \\
\text { height }\end{array}$ & $\begin{array}{l}\text { snout } \\
\text { height }\end{array}$ & $\begin{array}{l}\text { height maxillary } \\
\text { horizontal ramus, } \\
\text { snout height }\end{array}$ \\
\hline $\begin{array}{l}\text { increase } \\
\text { skull } \\
\text { height }\end{array}$ & $\begin{array}{l}\text { height } \\
\text { orbital \& } \\
\text { laterotemp } \\
\text { oral } \\
\text { fenestrae }\end{array}$ & $?$ & $\begin{array}{l}\text { enlarged } \\
\text { postorbital } \\
\text {, dorsal } \\
\text { shift bony } \\
\text { naris }\end{array}$ & $?$ & $\begin{array}{l}\text { caudally } \\
\text { inclined } \\
\text { lacrimal, } \\
\text { postorbital } \\
\text { enlarged }\end{array}$ & $\begin{array}{l}\text { lacrimal } \\
\text { ventral } \\
\text { ramus size, } \\
\text { postorbital } \\
\text { region } \\
\text { height, } \\
\text { angulation } \\
\text { between } \\
\text { maxilla and } \\
\text { jugal } \\
\end{array}$ & $\begin{array}{l}\text { lacrimal } \\
\text { ventral } \\
\text { ramus } \\
\text { size, } \\
\text { postorbit } \\
\text { al region } \\
\text { height, } \\
\text { dorsal } \\
\text { shift }\end{array}$ & $\begin{array}{l}\text { height orbital \& } \\
\text { laterotemporal } \\
\text { fenestrae, caudally } \\
\text { inclined lacrimal, } \\
\text { postorbital } \\
\text { enlarged, } \\
\text { postorbital region } \\
\text { height, angulation } \\
\text { between maxilla } \\
\text { and jugal }\end{array}$ \\
\hline $\begin{array}{l}\text { increase } \\
\text { in } \\
\text { adductor } \\
\text { chamber } \\
\text { size }\end{array}$ & $\begin{array}{l}\text { length } \\
\text { orbital } \\
\text { fenestra }\end{array}$ & $\begin{array}{l}\text { length } \\
\text { orbital } \\
\text { fenestra }\end{array}$ & $\begin{array}{l}\text { length } \\
\text { orbital } \\
\text { fenestra }\end{array}$ & $\begin{array}{l}\text { length } \\
\text { orbital } \\
\text { fenestra }\end{array}$ & $\begin{array}{l}\text { length } \\
\text { orbital } \\
\text { fenestra }\end{array}$ & $\begin{array}{l}\text { length } \\
\text { orbital } \\
\text { fenestra }\end{array}$ & $\begin{array}{l}\text { length } \\
\text { orbital } \\
\text { fenestra }\end{array}$ & $\begin{array}{l}\text { length orbital } \\
\text { fenestra }\end{array}$ \\
\hline $\begin{array}{l}\text { increase } \\
\text { in skull } \\
\text { width }\end{array}$ & $\begin{array}{l}\text { width } \\
\text { frontopari } \\
\text { etal region }\end{array}$ & $\begin{array}{l}\text { width } \\
\text { frontopariet } \\
\text { al region, } \\
\text { width snout }\end{array}$ & $?$ & $\begin{array}{l}\text { width } \\
\text { snout }\end{array}$ & $?$ & $?$ & $?$ & $\begin{array}{l}\text { width } \\
\text { frontoparietal } \\
\text { region, width snout }\end{array}$ \\
\hline $\begin{array}{l}\text { antorbital } \\
\text { fossa } \\
\text { length } \\
\end{array}$ & $?$ & $?$ & decrease & increase & decrease & decrease & decrease & $?$ \\
\hline $\begin{array}{l}\text { rostrocaud } \\
\text { al jaw } \\
\text { joint } \\
\text { position }\end{array}$ & caudal & $?$ & rostral & caudal & caudal & rostral & caudal & $?$ \\
\hline $\begin{array}{l}\text { dorsoventr } \\
\text { al jaw } \\
\text { joint } \\
\text { position }\end{array}$ & ventral & $?$ & ventral & dorsal & no change & ventral & ventral & $?$ \\
\hline $\begin{array}{l}\text { increase } \\
\text { of } \\
\text { mandible } \\
\text { height }\end{array}$ & $?$ & $\begin{array}{l}\text { height } \\
\text { mandible \& } \\
\text { emf }\end{array}$ & $?$ & $?$ & $?$ & $?$ & $?$ & $\begin{array}{l}\text { height mandible \& } \\
\text { emf }\end{array}$ \\
\hline $\begin{array}{l}\text { tooth } \\
\text { number }\end{array}$ & $\begin{array}{l}\text { increase } \\
\text { maxillary } \\
\text { teeth }\end{array}$ & $?$ & $?$ & $?$ & $?$ & $?$ & $?$ & $\begin{array}{l}\text { increase maxillary } \\
\text { teeth }\end{array}$ \\
\hline bite force & $?$ & increase & $?$ & $?$ & $?$ & $?$ & $?$ & increase \\
\hline $\begin{array}{l}\text { deltopecto } \\
\text { ral crest }\end{array}$ & $?$ & $?$ & $?$ & $\begin{array}{l}\text { enlarge } \\
\text { d crest }\end{array}$ & $?$ & $?$ & $?$ & enlarged crest \\
\hline $\begin{array}{l}\text { trochanter } \\
\text { ic crest }\end{array}$ & $?$ & $?$ & $?$ & $\begin{array}{l}\text { enhance } \\
\text { d crest }\end{array}$ & $?$ & $?$ & $?$ & enhanced crest \\
\hline
\end{tabular}


Table 24(on next page)

Predicted LAG counts for Tyrannosaurus rex specimens extrapolated from the ontogram.

Estimated ages are indicated by a tilde $(\sim)$, histologically aged specimens are in boldface. 
1

\begin{tabular}{ll}
\hline Specimen & Age (years) \\
\hline LACM 28471 & $\mathbf{2}$ \\
\hline AMNH FARB 5050, CMNH 7541, DDM 344.1 & $\sim 3-12$ \\
\hline BMRP 2002.4.1 & $\mathbf{1 3}$ \\
\hline BMRP 2006.6.4 & $\mathbf{1 5}$ \\
\hline RSM 2990.1, LACM 23845 & $\sim 15-17$ \\
\hline MOR 1125, TMP 1981.006.0001 & $\mathbf{1 8}$ \\
\hline AMNH FARB 5117, LACM 150167 & $\sim 18-22$ \\
\hline TMP 1981.012.0001 & $\mathbf{2 2}$ \\
\hline UWBM 99000 & $\sim 22$ \\
\hline AMNH FARB 5027, AMNH FARB 5029, BM R7994, CM 9380, MOR 008, & $\sim 23-27$ \\
MOR 555, MOR 980, MOR 1131, MOR 2822, LACM 23844, NMMNH P- \\
3698, RSM 2523.8, SDSM 12047, UCMP 118742, UMNH 11000, UWGM \\
181 \\
\hline FMNH PR2981 \\
\hline
\end{tabular}

2

3 